\title{
Biomechanical Analysis of the Stroking Cycle in the Sport of Sledge Hockey
}

by

Alicia Maria Gal, B.P.H.E., P.S.F.F.

A thesis submitted to the Faculty of Graduate and Postdoctoral Affairs in partial fulfillment of the requirements for the degree of

Masters of Applied Science

in

Biomedical Engineering

Carleton University

Ottawa, Ontario

(C) 2017, Alicia Maria Gal 


\section{Abstract}

The objective of this thesis is to investigate the stroking cycle used to produce skating in the sport of sledge hockey identifying possible performance enhancing stroke mechanics and potential skating introduced mechanisms of injury. Sledge hockey is a full body contact para-sport, where the skating motion is produced solely from the upper limbs using two sticks with picks that make contact with the ice surface. The research presented is comprised of two studies. Results are first of its kind for the sport of sledge hockey. Study 1 investigates free fall downward poling in sledge hockey at three drop heights for three fixed elbow angles (flexed, mid, and extended). An anatomically correct (mesomorph male) mechanical prototype $(S L A M-80)$, with a solid-static upper limb-stick system (solid segments linked with fixed joints) produces a free fall downward pole upon release of the support strap. Results indicate an extended elbow produces the least increase in shoulder joint reaction force at the most propulsive stick angle. Results suggest extending the elbow is slightly more efficient $(0.02 \mathrm{~N} / \mathrm{W}$, Newton-Watt $)$ when producing propulsive forces during limb extension (downward pole) compared to a flexed elbow. Overall, free fall downward poling in sledge hockey produces 2.0 times bodyweight $(B W)$ upon impact. Study 2 investigates task naïve double poling for a start stroke, mid stroke, and restricted stroke (mimics Study 1) during off-ice skating. Participants propelled themselves through a motion capture -4 force plate system. Results indicate an initial spiking force occurs at stick impact $\leq 3.1$ times $B W$ with only $\leq 0.4$ times $B W$ of force at push-off. Stick initial impact was determined to be a potential mechanism for injury. Validation of $S L A M-80$ was provided from Study 2 results permitting future investigations of spiking impact forces without the use of human participants. Stroke kinematics (i.e., elbow joint angles, and stick 
impact and push-off angles) appear to be important factors for producing enhanced skating in sledge hockey. Interpretations from the results are provided suggesting mechanisms of potential injury that are introduced from skating in order to improve the understanding for injury prevention within the sport of sledge hockey. 


\section{Acknowledgements}

First and foremost, I would like to thank my supervisors, Dr. Adrian D.C. Chan and Dr. Dean C. Hay. Their ability to provide knowledge, challenges, and support throughout this adventure was immeasurable and irreplaceable.

Next, I would like to thank my research assistant, Marcus Haefele. Through it all, for better or worse, we survived with smiles on our faces. Thank you, Mohamed Abdelazez, for providing unconditional support, knowledge, and assistance throughout this thesis.

Dr. Mario Lamontagne and Dr. Daniel Benoit for the use of the University of Ottawa Human Movement Biomechanics Laboratory. Erik Kowalski for his assistance and problem solving during data collection (Human Movement Biomechanics Laboratory). Thank you to my participants for volunteering their time to contribute to the sledge hockey database.

Finally, I would like to thank my family for their continuous support throughout my academic adventure. Specifically, I would like to thank my father, Jozef W. Gal, for all the late night proofreading and phone calls. Thank you for sharing your love of life-long learning with me. To my mother, P. II. Anne Gal, for always encouraging me to follow my dreams no matter the direction. 


\section{Statement of Originality}

This thesis presents the work of the author, under the supervision of Dr. Adrian D. C. Chan (Carleton University) and Dr. Dean C. Hay (Nipissing University), completed at Carleton University and the University of Ottawa for the degree of M.A.Sc. in Biomedical Engineering. The author of this thesis conducted experiment design and development including all equipment design and/or modification, data collection and analysis, and result composition and presentation of the below identified publications. The author's supervisors provided guidance and feedback regarding of the above-mentioned. Many of these results have been presented in conference publications. What follows is a summary of these publications.

A.M. Gal, D.C. Hay, \& A.D.C. Chan, "2 and 3-dimensional biomechanical analysis of the linear stroking cycle in the sport of sledge hockey: Glenohumeral joint kinematic, kinetic and surface EMG muscle modeling on and off ice", in Proceedings of 13th International Symposium on 3D Analysis of Human Movement (3D AHM), Lausanne, Switzerland, 2014, pp. 108-111. ISBN 9782880748562.

This conference paper described preliminary evidence of the biomechanics required to perform the linear stroking cycle within the sport of sledge, on and off ice. Evidence from video analysis (on-ice), motion capture (off-ice), and surface electromyography (on/off ice) was used to develop the motivations and methodology used to create this thesis as described in Chapter 1 and Chapter 4, respectively. The paper was presented by the author as a 1-minute (single slide) introduction and poster at the $13^{\text {th }}$ International Symposium on 
3D Analysis of Human Movement (3DAHM), in Lausanne, Switzerland.

A.M. Gal, A.D.C. Chan \& D.C. Hay, "Validating a solid-static single-armed male prototype tasked to produce dynamic movement from the shoulder through the preparation phase", in International Federation for Medical and Biological Engineering (IFMBE) Proceedings, World Congress on Medical Physics and Biomedical Engineering (IUPESM), Toronto, Canada, 2015, vol. 51, pp. 1146-1149. doi:10.1007/978-3-31919387-8_278.

This conference paper validated the architectural and mechanical design of the prototype used to mimic an average male's upper limb movement (2-dimensional) during the downward poling phase in the sport of sledge hockey. This prototype, its purposes, and evidence (motion capture and force plate analyses) used within this thesis are further discussed in Chapter 3. This paper was presented by the author as an oral presentation at the 2015 World Congress on Medical Physics and Biomedical Engineering (IUPESM), in Toronto, Canada.

A.M. Gal, A.D.C. Chan \& D.C. Hay, "Investigating the seated double poling cycle: Identifying baseline measures of the preparation phase", in $33^{\text {rd }}$ International Conference on Biomechanics in Sport (ISBS), Poitiers, France, 2015, pp. 1-4. sciencesconf.org:isbs2015:59230. 
This conference paper presented results from the primary data involved with test-retest validation for the prototype during the downward poling cycle within the sport of sledge hockey. Results provided kinematic (motion capture) and kinetic (force plate) data. Test and retest data present similar conclusions, retest results are outlined in Chapter 3 . This paper was presented by the author as a 5-minute ( 3 slides) introduction and poster at the $33^{\text {rd }}$ International Conference on Biomechanics in Sport (ISBS), in Poitiers, France.

What follows is a summary of conference presented unpublished abstracts.

A.M. Gal, A.D.C. Chan \& D.C. Hay, " Propulsion in sledge hockey: A biomechanical analysis to define gait", in Own the Podium SPorts INnovation (SPIN) Summit, Montreal, Canada, 2014.

This conference abstract described introductory parameters involved with the biomechanics required to perform a straight-line forward stroking cycle within the sport of sledge off-ice. Evidence comparing on-ice identified cycle phases with off-ice kinematic (motion capture) and kinetic (surface electromyography and force plates) results were used to design the prototype and methodologies created for this thesis as described in Chapter 3. The abstract was presented by the author as a poster at Own the Podium Sports Innovation (SPIN) Summit, in Montreal, Canada.

A.M. Gal, A.D.C. Chan \& D.C. Hay, "Baseline impact reaction forces in a seated downward short pole free-drop: A biomechanical investigation of elbow angle upon impact 
in the sport of sledge hockey", in Own the Podium SPorts INnovation (SPIN) Summit, Toronto, Canada, 2015.

This conference abstract presented results from the retest data involved with test-retest validation for the prototype during downward poling within the sport of sledge hockey. Results are presented in Chapter 3 with further publications in progress. The abstract was presented by the author as a poster at Own the Podium Sports Innovation (SPIN) Summit, in Toronto, Canada.

A.M. Gal, A.D.C. Chan \& D.C. Hay, "Shoulder joint produced weight-bearing mobility: Defining gait", in Women in Science and Engineering (WISE) National Conference (WISENC), Toronto, Canada, 2015.

This conference abstract presented the motivation and methodology used to correctly identify gait in an analogous form as outlined by gold-standard hip gait analysis (walking at the shoulders). Results represented the importance of understanding the downward poling cycle within the sport of sledge hockey to improve the quality of life for shoulderdependent populations as described in Chapter 2. This abstract was presented by the author as a poster at the Women in Science and Engineering National Conference (WISE-NC), in Toronto, Canada.

A.M. Gal, A.D.C. Chan \& D.C. Hay, "Differences between sagittal plane static start and mid-cycle stroke biomechanics during skating for the sport of sledge hockey: Task naïve 
population", in Own the Podium SPorts INnovation (SPIN) Summit, Calgary, Canada, 2016.

This conference abstract presented results for torso and stick angles from task naïve adult male athletes with single or both dominant limb sets (no upper limb stroking expertise). Task naïve biomechanics were interpreted identifying areas of difficulty during the propulsion phase for a start and mid-cycle stroke within the sport of sledge hockey off-ice. Results are presented in Chapter 4 with further publications in progress. The abstract was presented by the author as a poster at Own the Podium Sports Innovation (SPIN) Summit, in Calgary, Canada.

A.M. Gal, A.D.C. Chan \& D.C. Hay, "Investigation of the relationship between peak impact and push-off reaction forces, and potential upper limb overuse/overloading injuries introduced from skating in the sport of sledge hockey", in Own the Podium SPorts INnovation (SPIN) Summit, Calgary, Canada, 2016.

This conference abstract presented results for peak impact and push-off ground reaction forces produced during task naïve skating in the sport of sledge hockey off-ice. Results were compared to parallel upper and lower limb impact researches in order to predict skating introduced potential for injury. Results are presented in Chapter 4 with further publications in progress. The abstract was presented by the author as a poster at Own the Podium Sports Innovation (SPIN) Summit, in Calgary, Canada. 


\section{Table of Contents}

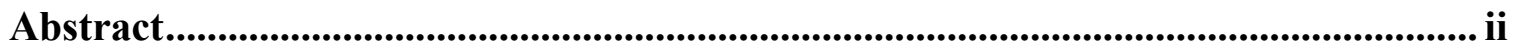

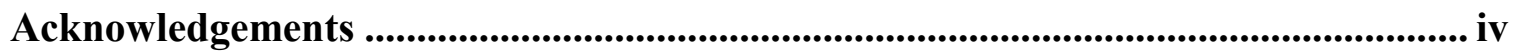

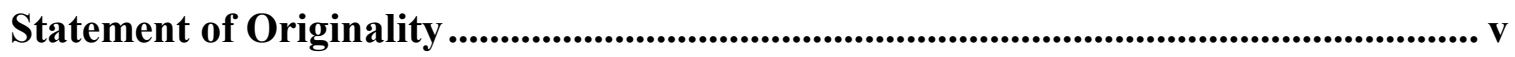

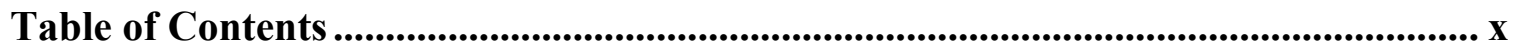

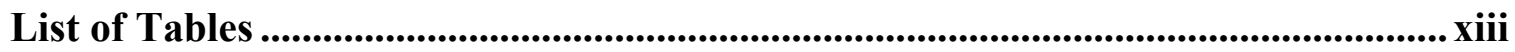

List of Figures...........................................................................................................

List of Abbreviations ................................................................................................... xviii

1 Chapter: Introduction ........................................................................................ 1

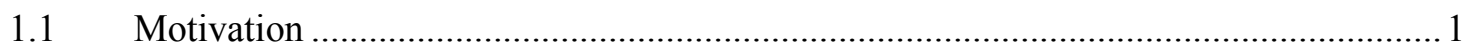

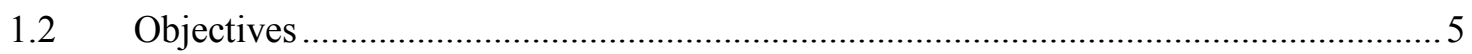

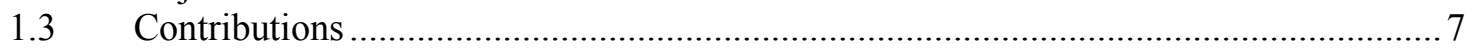

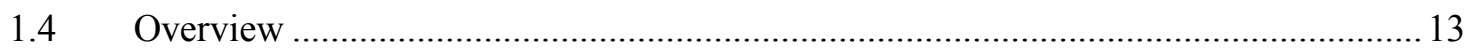

2 Chapter: Background \& Literature Review .............................................................. 16

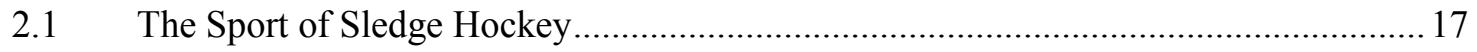

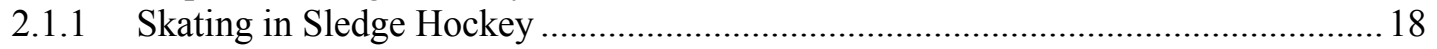

2.1.2 Theoretical, Mechanical, and Anatomical Double Poling Locomotion ....................22

2.1.3 Cross-Country Sit-Skiing: Biomechanical Review ...............................................24

2.1.4 Upright Cross-Country Skiing: Biomechanical Review .......................................25

2.1.5 Existing Research on the Sport of Sledge Hockey .................................................... 32

2.1.6 Increased Force Production versus Decreased Structural Integrity ...........................35

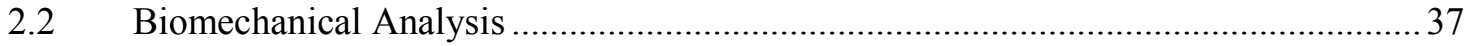

2.2.1 Kinematics Acquired Through Motion Capture ...................................................... 37

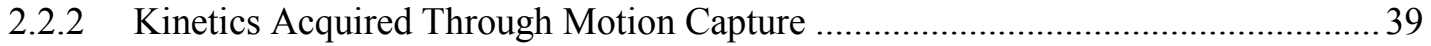

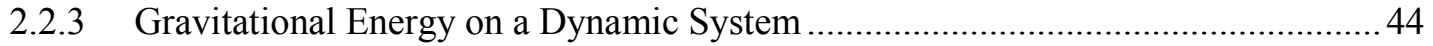

2.2.4 Injury and the Internal Architecture of the Dynamic System ................................. 45

2.2.5 Kinetics Acquired Through Surface Electromyography …..................................... 48

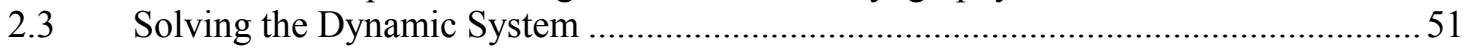

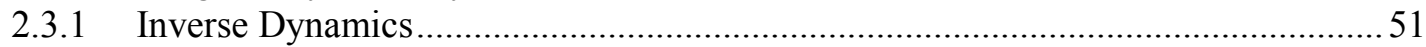

2.3.2 Ground Reaction Forces Acquired Through Force Plates.......................................52

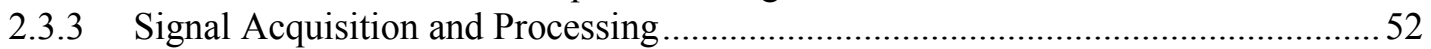

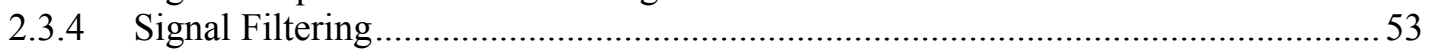

2.3.5 Processing and Publication of Acquired Signals................................................. 54

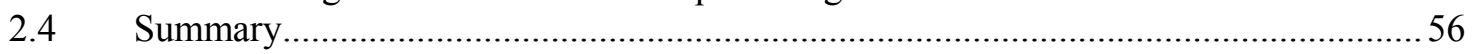

3 Chapter: Study 1: Solid-Static System......................................................... 58

3.1 A Relationship between Free Fall Downward Poling in Sledge Hockey and Propulsive

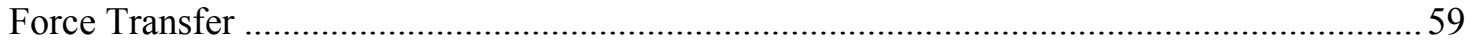

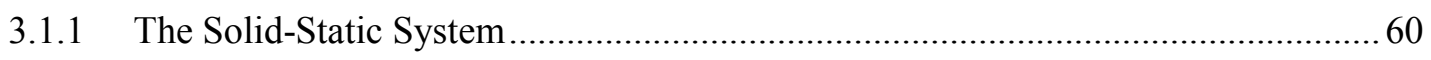

3.1.2 Baseline Deviations and Skating Initiation in Sledge Hockey ................................. 61 
3.1.3 Understanding Lever Arm to Enhance Force Production Initiation in Sledge Hockey 62

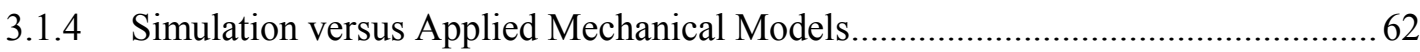

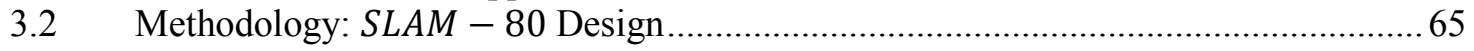

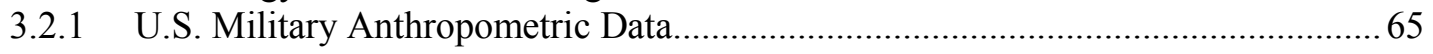

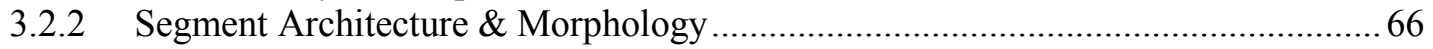

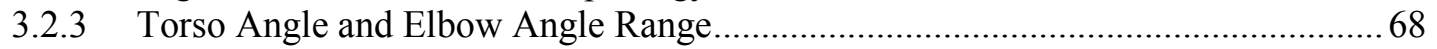

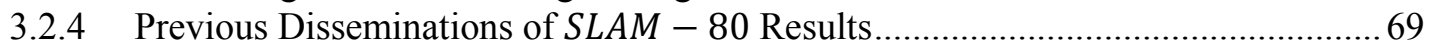

3.3 Methodology: Acquisition Apparatus and SLAM - 80 Protocol................................. 71

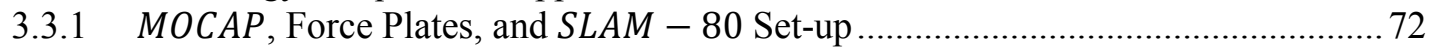

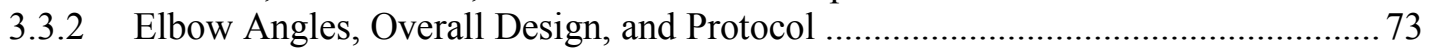

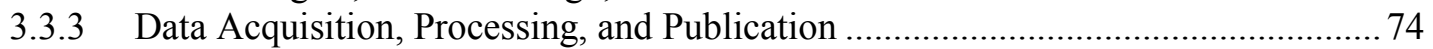

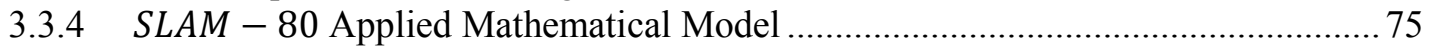

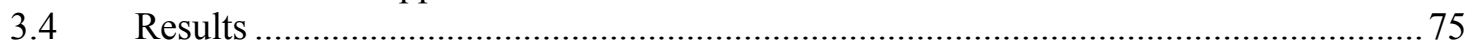

3.4.1 Sagittal Plane Pick Impact Angles for Free Fall Downward Poling in Sledge

Hockey 76

3.4.2 Impact Force for Free Fall Downward Poling in Sledge Hockey ............................ 78

3.4.3 Estimated Joint Reaction Force at the Shoulder for Free Fall Downward Poling in

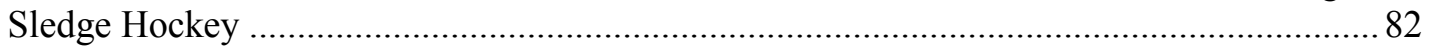

3.4.4 Performance and Potential Injury Indicators for Skating in Sledge Hockey............. 84

3.4.5 Estimated Limb Segment Peak Power invloving the Shoulder for Free Fall

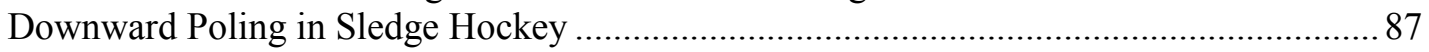

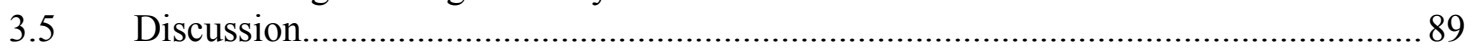

3.5.1 SLAM - 80 and Future Research Involving Skating in Sledge Hockey.................. 92

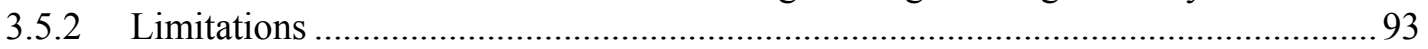

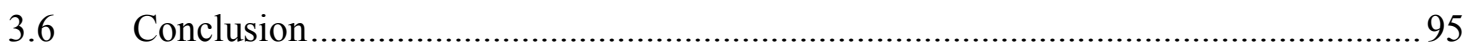

4 Chapter: Study 2: Task Naïve Off-Ice Skating in Sledge Hockey ....................... 96

4.1 Task Naïve Population and Sledge Hockey Skating Analysis .................................... 97

4.2 Methodology: Participant Recruitment and Preparation ............................................99

4.2.1 Research Ethic Boards Approval and Requirements ............................................99

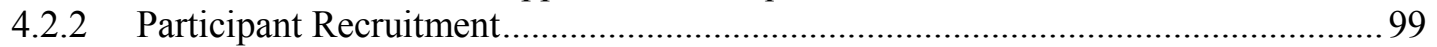

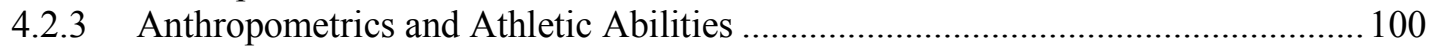

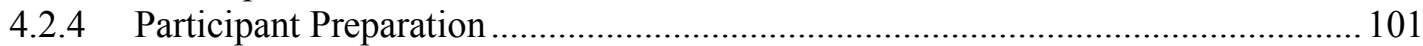

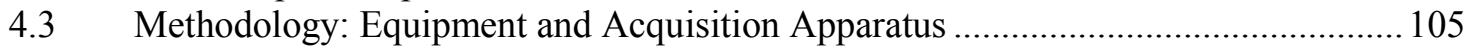

4.3.1 Off-Ice Modifications to On-Ice Sledge Hockey Equipment .................................. 105

4.3.2 Elbow Brace, Wrist Restriction, and Restricted Angles ....................................... 109

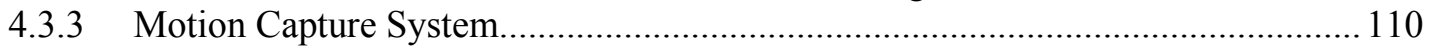

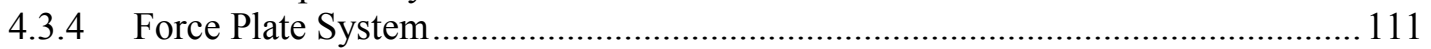

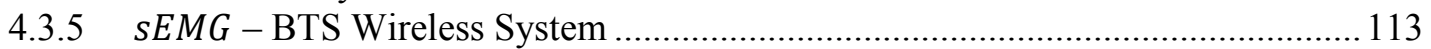

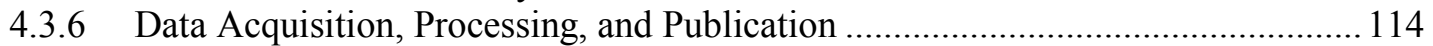

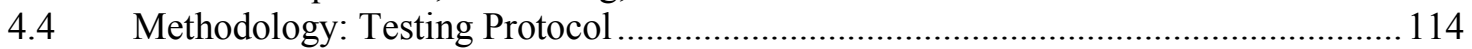

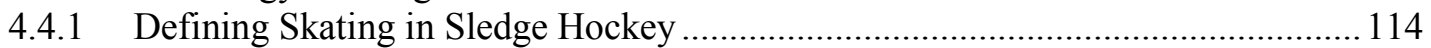

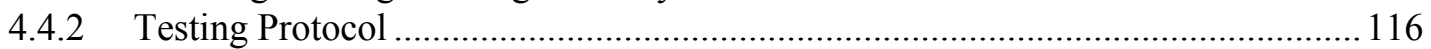

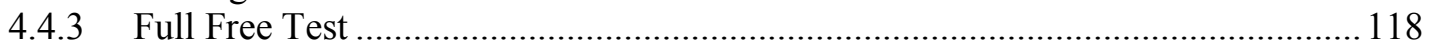

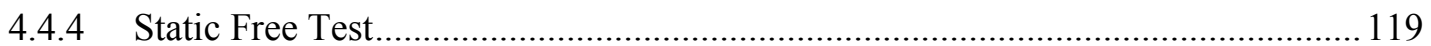

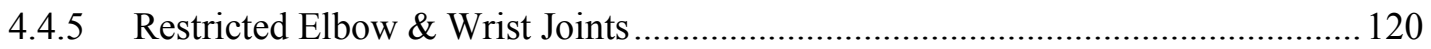

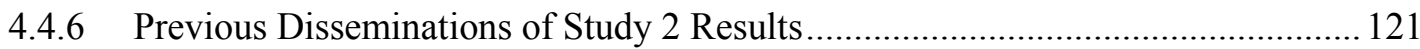

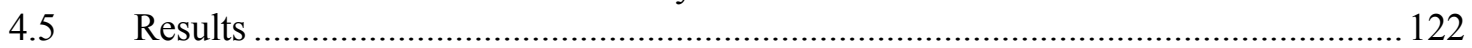

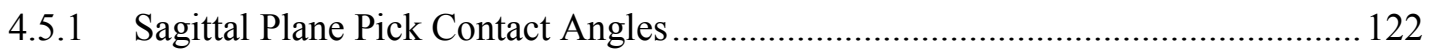


4.5.3 Estimated Joint Reaction Force at the Shoulder for a Downward Pole in Sledge

Hockey 131

4.5.4 Performance and Potential Injury Indicators for Skating in Sledge Hockey .......... 133

4.5.5 Estimated Limb Segment Peak Power at the Shoulder ........................................ 135

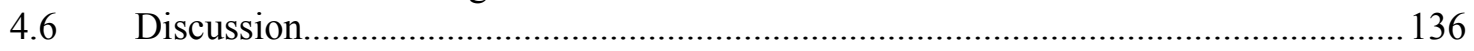

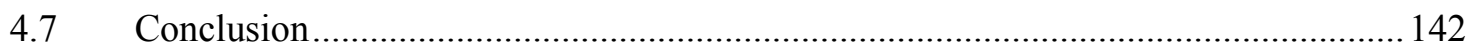

5 Chapter: Aggregated Results............................................................................... 144

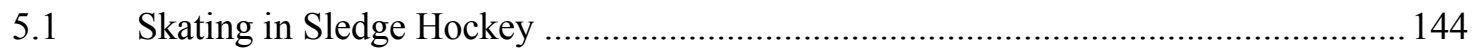

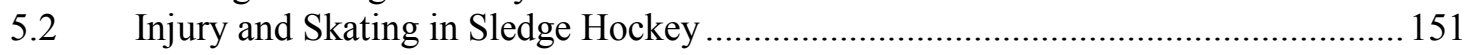

6 Chapter: Conclusion ................................................................................ 153

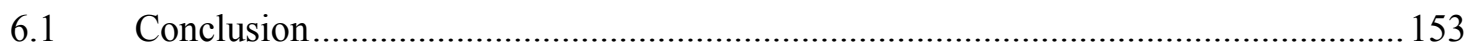

7 Chapter: Limitations and Amendments ....................................................... 157

7.1 Skating in Sledge Hockey, Potential for Injury, and Future Research ...................... 157

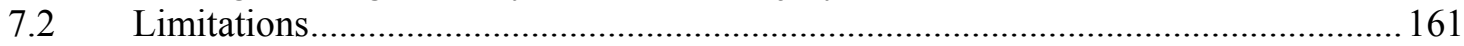

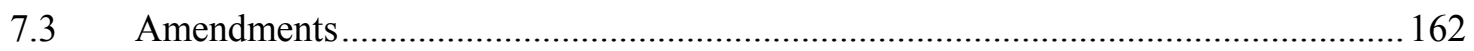

Appendix A Background and Literature Review ......................................................... 163

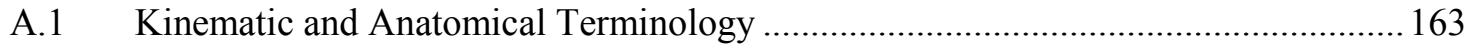

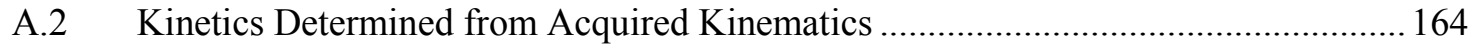

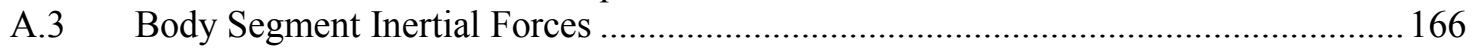

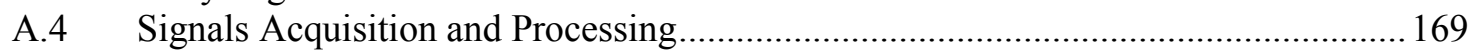

Appendix B ............................................................................................................................. 171

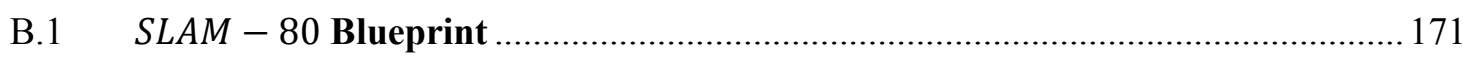

Appendix C Theoretical Proofs - Mathematical Models ........................................ 172

C.1 Inverse Dynamics Model for the Shoulder in the Sport of Sledge Hockey................ 172

C.2 Power for the GHJ produced from a downward pole in the sport of sledge hockey .. 196

Appendix D Research Ethics Approval and Requirements ........................................ 197

D.1 Carleton University Research Ethics Board Approval ............................................. 197

D.2 University of Ottawa Health Sciences and Science Research Ethics Board Approval 198

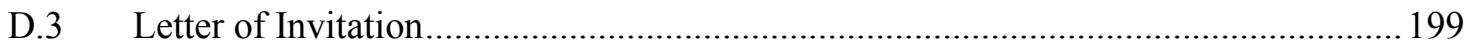

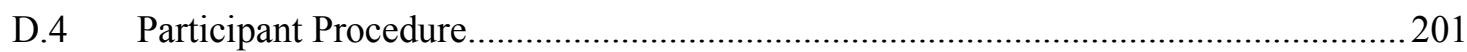

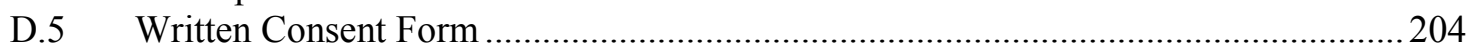

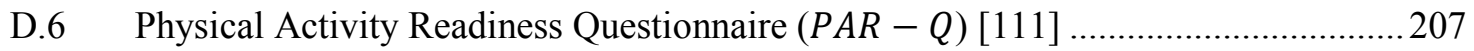

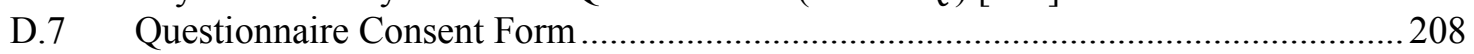

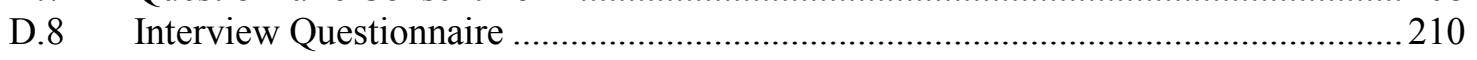

Appendix E Amendments................................................................................................ 212

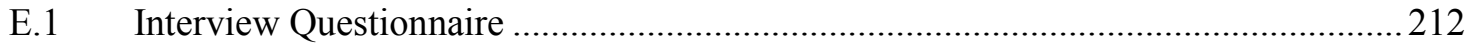

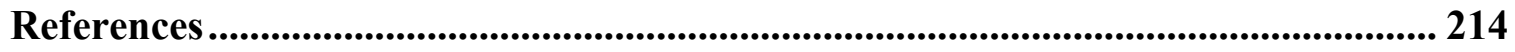




\section{List of Tables}

Table 2.1 Cross-country skiing double poling kinematic and kinetic data 32

Table 2.2 Global co-ordinate relationships within biomechanical analysis 38

Table 2.3 Muscles of interest for this research scope involved with shoulder and elbow movements during skating in sledge hockey 50

Table 3.1 Advantages and disadvantages of computer simulation [98, p. 377]. 64

Table 3.2 SLAM - 80 anthropomorphic measurements [20], [22], [67] 66

Table 3.3 Segment architecture for SLAM - 80 [20], [22], [67] 68

Table 3.4 Pick impact angles for all SLAM - 80 trials 77

Table 3.5 Component impact force analysis for SLAM - 80 79

Table 3.6 Resultant impact force analysis for $S L A M-80 \quad 80$

Table 3.7 Mean of all resultant impact forces produced by $S L A M-80 \quad 80$

Table 3.8 Estimated $J R F_{\text {Shoulder }}$ from $S L A M-80$ at peak impact force 83

Table 3.9 Estimated torque produced from $S L A M-80$ at peak impact force $\quad 84$

Table 3.10 Impulse for total force plate contact produced by $S L A M-80$ free fall downward poling $\quad 87$

Table 3.11 Estimated limb segment peak power produced from $S L A M-80$ at peak impact

Table 3.12 Estimated limb segment peak specific power produced from $S L A M-80$ at peak impact, normalized by body mass 88

Table 4.1 Anthropometric measurements and athletic abilities for participants in Study 2

Table 4.2 ASIS to Rib 10 distance for participants in Study 2

Table 4.3 Stick marker measurements 109

Table 4.4 sEMG electrode replacements for participants in Study $2 \quad 114$

Table 4.5 Pick impact and push-off angles for naïve of-ice skating in sledge hockey 123

Table 4.6 Left vs right limb propulsion phase resultant impact forces for Study $2 \quad 128$

Table 4.7 Left vs right limb propulsion phase push-off forces for Study 2

Table 4.8 Estimated $J R F_{\text {Shoulder }}$ for human participants at peak impact force $\quad 132$

Table 4.9 Estimated torque produced from human participants at peak impact 134

Table 4.10 Estimated limb segment specific power produced from human participants at peak impact, normalized by bodyweight 136

Table 7.1 Observable gait parameters 159

Table C. 1 Standard CoM calculation table used in classic mechanics to solve CoM of a geometric shape creating a sledge hockey stick 180

Table C.2 CoM inertia and radius of gyration used in classic mechanics for each geometric shape creating a sledge hockey sticks 


\section{List of Figures}

Figure 1.1 On-ice Paralympic sledge hockey depicting various phases of the stroking cycle and skating styles. Player No 2 is at rest. Player No 11 is at pick-off for his right arm, and in the recovery phase for his left (asymmetrical skating). Player No 4 is within the newly proposed preparation phase, and shows approximate bilateral symmetry (see Chapter 2). All players in motion (No 4, 11, 16) have flexed torsos [12].

Figure 2.1 A typical right wooden sledge hockey stick with on-ice picks (to scale - picks start at $1.00 \mathrm{~cm})$.

Figure 2.2 MOCAP computer regenerated single gait cycle showing phase boundaries for the sport of sledge hockey (a) Recovery termination - Preparation initiation (pick $y$ max), (b) Propulsion impact (pick $y=0 \mathrm{~m}$ at $t_{\text {Impact }}$ ), (c) Propulsion Recoil - Recovery initiation $\left(y_{i+1}\right.$, where $y_{i}=0 \mathrm{~m}$ at $\left.t_{\text {Recoil }}\right)$, and (d) Recovery termination - Preparation initiation (pick $y$ max). Yellow - head and torso, pink - Rib 10, lower body, and sledge, red - right limb, green - left limb, and purple - sticks.

Figure 2.3 MOCAP depiction of the GHJ as a third-class lever during the poling cycle in the sport of sledge hockey (force arrow represents direction not magnitude). Pick is point of impact where shoulder joint-centre is point of applied reaction forces. From this, it is clear that an elongated moment arm is created during skating in sledge hockey, foreshadowing potential of overloading injuries.

Figure 2.4 Angular range and moment arm length involving elbow flexion and extension, a third-class lever [72]. Since mechanical advantage represents the effectiveness of the lever moving the resistance, a third-class lever, favours the resistance-arm or limb over the force-arm or muscle insertion. Specifically, this figure depicts the importance of the moment arm in relation to the overall system at various instances during a dynamic movement. From this, limb mechanics can be identified as vital components in order to promote optimal force generation and/or transduction. Moment arm (black dash), lever arm (black solid), elbow flexion moment arm (red dash), elbow extension moment arm (green dash), and force arrow (blue arrow).

Figure 2.5 a) Anatomical planes and axes, and (b) Anatomical movements [73], [74]. This thesis focuses biomechanical investigation primarily concerning the sagittal plane ( (a) - red). Motions of importance to skating in sledge in this plane include flexion and extension of the upper limbs and torso ( (b) - first two top and bottom first, respectively).

Figure 2.6 Architecture of the shoulder complex and upper limb within the human body [75]. The shoulder girdle is comprised of the clavicle and scapula. The shoulder girdle in conjunction with the humerus creates the $G H J$, and in combination with the thoracic cage, sternoclavicular joint, acromioclavicular joint, and scapulothoracic joint the shoulder complex exists. The anterior superior component of the GHJ socket consists of the clavicle, a thin elongated S-shaped bone composed of mostly cortical bone running horizontally to the superior thoracic cage. The scapula, an inverted triangular bone, again mostly composed of cortical bone, is the posterior component of the socket. The humeral head of the long bone known as the humerus (superficial cortical bone accompanied by deep trabecular bone) creates the ball.

Figure 2.7 Shoulder complex skeletal muscle orientations of particular interest for this research scope involving locomotors and stabilizers producing skating in sledge hockey [95]. Skeletal muscles originate at a bone-tendon interface (origin), produce force to 
stabilize and/or mobilize their adjoining bone located at the insertion (anchor). A note should be made that the rotator cuff is the primary shoulder stabilizer; however, due to its deep location $S E M G$ acquisition cannot occur.

Figure 3.1 SLAM - 80 horizontal start position (shoulder joint-centre to elbow jointcentre) located centrally in a 10-camera MOCAP system. Impact forces were obtained from a single force plate protected by a rubber wavy mat (black force plate closest to sledge). SLAM - 80 freestanding in the late preparation phase was tested.

Figure 3.2 (a) $S L A M-80$ foam prototype created to ensure task congruency and structural design. Key measurements included segment length (green) and joint-centre to joint-centre (yellow), and (b) Sagittal plane impact position for a typical SLAM - 80 trial. Pick contact was the only contact force acquired from the force plate.................. 68 Figure 3.3 MOCAP computer regenerated SLAM - 80 start heights and elbow angles: (a) Mid elbow above drop height, (b) Extended elbow horizon drop height, and (c) Flexed elbow below drop height start position.

Figure $3.4 S L A M-80 E 150^{\circ}$ trial three above drop height sagittal plane trajectories illustrating pick impact angle for downward poling; black - limb, blue - stick, solid $-t_{0}$, and dashed $t_{\text {peak }}$. Mid and extended elbows produced the most acute pick impact angles implying the most propulsive stick positions for stroke initiation in sledge hockey at a $40^{\circ}$ torso angle.

Figure $3.5 S L A M-80 E 150^{\circ}$ above drop height (trial three) resultant impact force curve (black solid), and peak $J R F_{\text {Shoulder }}$ (black dashed $=2021 \mathrm{~N}$ ) (left axis), and rate of force development (red solid) (right axis). Aggregation of these data represents force involved with the shoulder during free fall forward force transfer initiation in sledge hockey. ..... 81 Figure 3.6 SLAM - 80 peak impact force from free fall downward poling against the difference of $J R F_{\text {Shoulder }}$ and peak impact force for all elbow angles $\left(E 120^{\circ}-\mathrm{x}, E 135^{\circ}\right.$ *, and $E 150^{\circ}-\mathrm{o}$ ), and drop heights (above - blue, horizon - green, below - red). Mean values for each elbow angle are represented in black, and mean of all trials (black + ). Positive values represent an increase of force at the point of rotation caused from peak impact.

Figure 3.7 SLAM - 80 impulse against estimated torque about the shoulder for all elbow angles $\left(E 120^{\circ}-\mathrm{x}, E 135^{\circ}\right.$ - $^{*}$, and $E 150^{\circ}$ - o) and drop heights (above - blue, horizon green, below - red). Mean values for all elbow angles are represented in black, and mean of all trials (black + ). Positive torque represents flexion of the shoulder. 86 Figure 4.1 sEMG and marker locations for Study 2 as per Section 4.2.4 (a) Posterior, and (b) Anterior views. 104

Figure 4.2 Exploded view of the telescopic leg-rest and wheel addition designed for multiple participant use during off-ice sledge hockey skating analysis. 107 Figure 4.3 Indoor modified sledge hockey sledge used for skating analysis (a) Wheel configuration, and (b) Sagittal plane view.

Figure 4.4 Sledge hockey stick used for indoor analysis with general marker locations (shiny circles - two-sided adhesive). 108

Figure 4.5 LE101 elbow brace used for restricted trials. 110 Figure 4.6 Wrist flexion-restricting design for restricted tests promoting appropriate palm-stick interfaces. 110 Figure 4.7 Vicon MOCAP system configuration Human Movement Biomechanics Laboratory for a Static Free test trial. 
Figure 4.8 Aerial view of the force plate configuration used in Study 2, yellow arrow

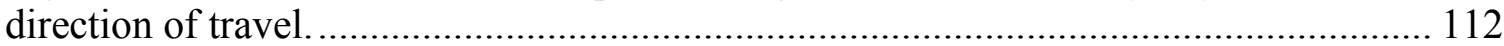

Figure 4.9 BTS bipolar wireless electrode used in Study 2..................................... 113 Figure 4.10 Full Free trial start position enabling mid stroke data to be collected upon force plate contact for Study 2 .

Figure 4.11 Static Free trial start position enabling start stroke data to be collected upon force plate contact for Study 2.

Figure 4.12 Restricted trial horizontal start position enabling impact data to be collected upon force plate contact for Study 2 (similar to Study 1).

Figure 4.13 Representation for two of the three propulsion phase stroking patterns observed during Study 2. Start stroke (left) and mid stroke (right), right limb (green) and stick (black) trajectories from $t_{0}$ (dotted) to $t_{\text {Recoil }}$ (dash), and at $t_{\text {Peak }}$ (solid). Motion of the stick is provided by the joint of the shaft (red) and pick of stick (blue). Every participant performed at least one pulling motion (right) during the propulsion phase, with one participant pulling every start stroke (C3). The final stroking pattern was a static held stick.

Figure 4.14 Generalized depiction of combined reaction force curves for mid stroke skating in sledge hockey off-ice (left stick - blue, right stick - red, impact body-sledge black, push-off body-sledge - grey). Left stick force curves indicate a more fluent stroke than the right (fluctuation). SLAM - 80 baseline for mean impact force $(1609 \mathrm{~N})$ is indicated in green.

Figure 4.15 Bosu ball double poling off-ice training exercise to increase comfort within the sledge bucket improving balance during force transfer (a) Pick contact through to (b) Pick-off.

Figure 5.1 Right stick start stroke submaximal impact force distributions by bodyweight for the propulsion phase in the sport of sledge hockey. Mediolateral force curve (top), horizontal force curve (middle), and vertical force curve (bottom). Fluctuating breakingpropulsive forces are observed in the middle plot. Smoother waveforms are proportional to improved force transfer.

Figure 5.2 Left stick mid stroke maximal impact force distributions by bodyweight for the propulsion phase in the sport of sledge hockey. Mediolateral force curve (top), horizontal force curve (middle), and vertical force curve. Fluctuating breaking-propulsive forces occur less within the mid stroke (middle plot). From this, this participant produced enhanced force transfer compared to the submaximal start stroke; starting from static stop requires more force.

Figure 5.3 Sagittal plane viewpoint anatomical perspective (spatial) illustrating SLAM-80 (blue) and musculoskeletal (red) produced downward poling for off-ice sledge hockey. Single elbow angle $135^{\circ}$ restricted trials is plotted $t_{0}$ to $t_{\text {Recoil }}$, and $S L A M-80$ single trial is plotted $t_{0}$ to $y_{\max }$ post $t_{\text {Recoil }}$ (depicts a uniform recoil trajectory post impact). Note: Musculoskeletal elbow marker was placed laterally on top of elbow brace creating a shortened forearm during sagittal plane observations...

Figure 7.1 Elbow joint-centre axel screw deformation as a results of impact forces from approximately 60 downward skating poles.

Figure C.1 Sagittal plane co-ordinates system for this model used to investigate skating in sledge hockey 
Figure C.2 Sagittal plane angle measurement definition with regards to the vector under

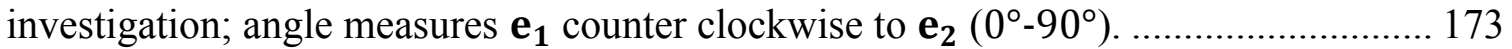
Figure C.3 Sagittal plane multi-segment alignment used in this model to investigate skating in sledge hockey; human participant marker location (blue), and joint location for calculations (red). Forearm and wrist are in neutral rotation in order to produce locomotion from the stick [68]. Markers outlined are required for below-mentioned manipulations; pick of stick $(P)$, joint of stick $(J)$, palm-stick interface $(H A N D)$, shoulder $\left(S^{\circ}\right)$, and $G H J$ local origin $\left(O S^{\circ}\right)$. Markers along the long axes are used in calculations for both studies. 174

Figure C.4 (a) Human participant marker locations for the upper limb (blue circles), and $G H J$-centre (red x), and (b) Sagittal plane shoulder marker $\left(S^{\circ}\right), G H J^{\circ}$, and upper arm CoM (ua) relationship for shoulder marker manipulations. The distance from upper arm marker to $G H J^{\circ}$ is a constant (green line), and the angle of the humerus can be calculated $(\alpha)$ from the elbow marker to the upper am marker. Then by trigonometry $G H J^{\circ}$ coordinates can be found (red $\mathrm{x}$ ).

Figure C.5 Sagittal plane representation of various random wrist angles; magenta neutral wrist radial deviation, cyan - neutral wrist ulnar deviation, and green neutral wrist along the vector created by the forearm. The distance from wrist to hand CoM is represented by A, assuming the hand-stick interface remains (hand does not move up and down the shaft). The distance of hand $C o M$ to joint marker $(J)$ is represented by $\mathrm{B}$, again assuming the hand-stick interface remains. From these relations the final side to the triangle can be solved, and interior angles determined via the Law of Cosine or Sine Law (blue triangle). $\tau$ is wrist-stick angle, and $\gamma$ wrist angle with respect to the forearm.... 179 Figure C.6 Assumed geometric shapes of a typical sledge hockey stick. CoM (yellow x) and radius of gyration for a sledge hockey stick can be solved using the outlined geometry. Assume rectangular widths equal ( $b_{\text {red }}=b_{\text {green }}$, where $b$ represents base) and local origin $(0,0)$ is at the purple $\mathrm{x}$.

Figure C.7 Sagittal plane upper limb representation at start position. Palm-stick interface is represented at $H A N D$ assuming the $H A N D$ is along the vector created from the forearm.

Figure C.8 Sagittal plane representation of the above-mentioned calculations creating the co -ordinates for the local origin $\left(S^{\circ}\right)$ for $S L A M-80$.......................................... 186 Figure C.9 A variation of the above-mentioned calculations for sagittal plane $S L A M-80$ analysis; drop height $-10^{\circ}$, elbow angle $135^{\circ}$, wrist-pick angle $45^{\circ}$.... 190 Figure C.10 Sagittal plane triangular relationships for $S L A M-80$ (thick black). Variations of combinations of outlined relationships are used to solve the system at pick $\mathrm{y}=0.0000 \mathrm{~m}$.

Figure C.11 Sagittal plane representation of the Pendulum Theory calculating $P_{0}$ to $P_{\text {Peak }}$ for $S L A M-80$. 191

Figure C.12 Free body diagram $(F D B)$ at peak resultant reaction force solving for force and torque at the wrist. $P(0,0)$ and unknowns are assumed positive as per Winter [96].

Figure C.13 FDB at peak resultant reaction force solving for force and torque at the elbow. $P(0,0)$ and unknowns are assumed positive as per Winter [96]. 195

Figure C.14 FDB at peak resultant reaction force solving for force and torque at the shoulder. $P(0,0)$ and unknowns are assumed positive as per Winter [96]. 


\section{List of Abbreviations}

\begin{tabular}{|c|c|}
\hline$a$ & Acceleration \\
\hline$A b v$ & Above Drop Height \\
\hline$A D L \mathrm{~s}$ & Activities of Daily Living \\
\hline$A P$ & Anterior-Posterior \\
\hline Blw & Below Drop Height \\
\hline$B W$ & Bodyweight \\
\hline C & Participant Identification Code (followed by a number) \\
\hline CoM & Centre of Mass \\
\hline DoF & Degree of Freedom \\
\hline$E$ & Elbow Angle Parameter (followed by a degree) \\
\hline$E M G$ & Electromyography \\
\hline$F$ & Force \\
\hline$F B D$ & Free Body Diagram \\
\hline$F F T$ & Fast Fourier Transform \\
\hline$I$ & Inertia \\
\hline$i$ & Index \\
\hline GHJ & Glenohumeral Joint \\
\hline GRF & Ground Reaction Force \\
\hline Horz & Horizon Drop Height \\
\hline$I S G$ & International Shoulder Group \\
\hline$J R F$ & Joint Reaction Force \\
\hline$L$ & Angular Momentum \\
\hline
\end{tabular}




\begin{tabular}{|c|c|}
\hline $\mathrm{L}$ & Left Limb \\
\hline$L C S s$ & Local Co-Ordinate Systems \\
\hline Long & Longitudinal \\
\hline$M$ & Moment \\
\hline$m$ & Mass \\
\hline $\operatorname{Max}$ & Maximal Effort \\
\hline Mid & Mid Stroke \\
\hline$M L$ & Mediolateral \\
\hline MOCAP & Motion Capture \\
\hline$M V I C$ & Maximal Voluntary Isometric Contraction \\
\hline$n$ & Sample Number \\
\hline$p$ & Linear Momentum \\
\hline$P$ & Power \\
\hline$P A R-Q$ & Participant Activity Readiness Questionnaire \\
\hline PREP & Preparation Phase \\
\hline$P R O$ & Propulsion Phase \\
\hline$P w D s$ & Persons with Disabilities \\
\hline$R$ & Reaction Force \\
\hline $\mathrm{R}$ & Right Limb \\
\hline$r$ & Distance of Force Applied to Point of Rotation \\
\hline$R E C$ & Recovery Phase \\
\hline$s$ & Distance \\
\hline$s E M G$ & Surface Electromyography \\
\hline
\end{tabular}




$\begin{array}{cl}\text { SLAM } 80 & \text { Sledge Arm Male }-80 \mathrm{~kg} \text { (prototype name) } \\ \text { Start } & \text { Signal to Noise Ration } \\ \text { Sub } & \text { Submaximal Effort } \\ t & \text { Time } \\ v & \text { Velocity } \\ W & \text { Work } \\ x & \text { Anterior-Posterior Axis } \\ y & \text { Longitudinal Axis } \\ z & \text { Mediolateral Axis }\end{array}$

Appendix C provides additional abbreviation definitions when necessary (appendix specific)

$\begin{array}{ll}\alpha & \text { Angular Acceleration } \\ \Delta & \text { Delta (difference/change) } \\ \omega & \text { Angular Velocity } \\ \rho & \text { Radius of Gyration } \\ \theta & \text { Measured Angle } \\ \tau & \text { Torque }\end{array}$

Appendix C provides additional abbreviation definitions when necessary (appendix specific) 


\section{Chapter: Introduction}

This chapter consists of four sections. Section 1.1 provides the motivation used to develop this thesis involving biomechanical investigation of skating in the sport of sledge hockey. Section 1.2 provides the thesis objectives. Section 1.3 summarizes the major contributions of this thesis in a sport-specific context, as well as transferrable interpretations towards the general shoulder-dependent population. Section 1.4 describes the organization of the remaining chapters within this thesis.

\subsection{Motivation}

The shoulder-dependent population in Canada is increasing due to injuries (e.g., military combat, workplace, athletic, and transportation accidents) and congenital disabilities [1], [2]. Shoulder-dependent is defined as the primary reliance on the shoulder joints for ambulation, which would typically occur from the hip joints, such as walking. Assisted devices like wheelchairs or crutches provide support to create shoulder-dependent bodyweight locomotion. Bodyweight locomotion introduces potential for injury caused by overuse and/or overloading the joints of the load-bearing limbs [3]-[6]. The level of severity of an overuse/overloading injury can be associated with the amount, duration, and/or how the forces are applied to the load-bearing joint [3], [5]-[7]. Analysis of the above-mentioned characteristics of applied forces can provide indictors for potential injury, as well as transferrable knowledge to improve injury prevention and rehabilitation practices [3]-[6]. 
Sledge hockey (Figure 1.1) is a shoulder-driven, on-ice para-sport, played sitting in a bucket (seat) on top of two width-adjustable skate blades [8]-[10]. Less skilled players use blades set wider apart for stability, whereas expert players use blades nearly touching for greater agility [8]. The bucket is attached to a metal-frame leg-rest equipped with a triangular balance point support underneath the footrest; regulations require a minimum length for the leg-rest regardless of its use or not [8]-[10]. The bottom of the bucket rests on the blades-chassis, which are roughly $10 \mathrm{~cm}$ in height from the ice surface [8]. Two shortened hockey sticks, equipped with picks at one end and elongated blades at the other, are used to propel the body and sledge across the ice surface, similar to double poling in cross-country skiing [8]-[10]. This contact phase is defined as propulsion, which occurs from initial pick contact to final pick push-off, and is the exclusive mechanical method for motion production within the sport [11].

As participants within the sport of sledge hockey have diverse physical capacities, the pushing motion can take many forms. Research concerning task performance involving persons with disabilities $(P w D s)$ can provide insight to improve task delivery based upon the effect(s) of the observed limitation(s). Limitation(s) caused by impairment(s) can negatively affect activities of daily living ( $A D L s)$, decreasing quality of life for $P w D s$. Biomechanical investigations incorporating impairment evaluation can produce evidence used to derive manipulation(s) for improved task production, in turn improving quality of life. 


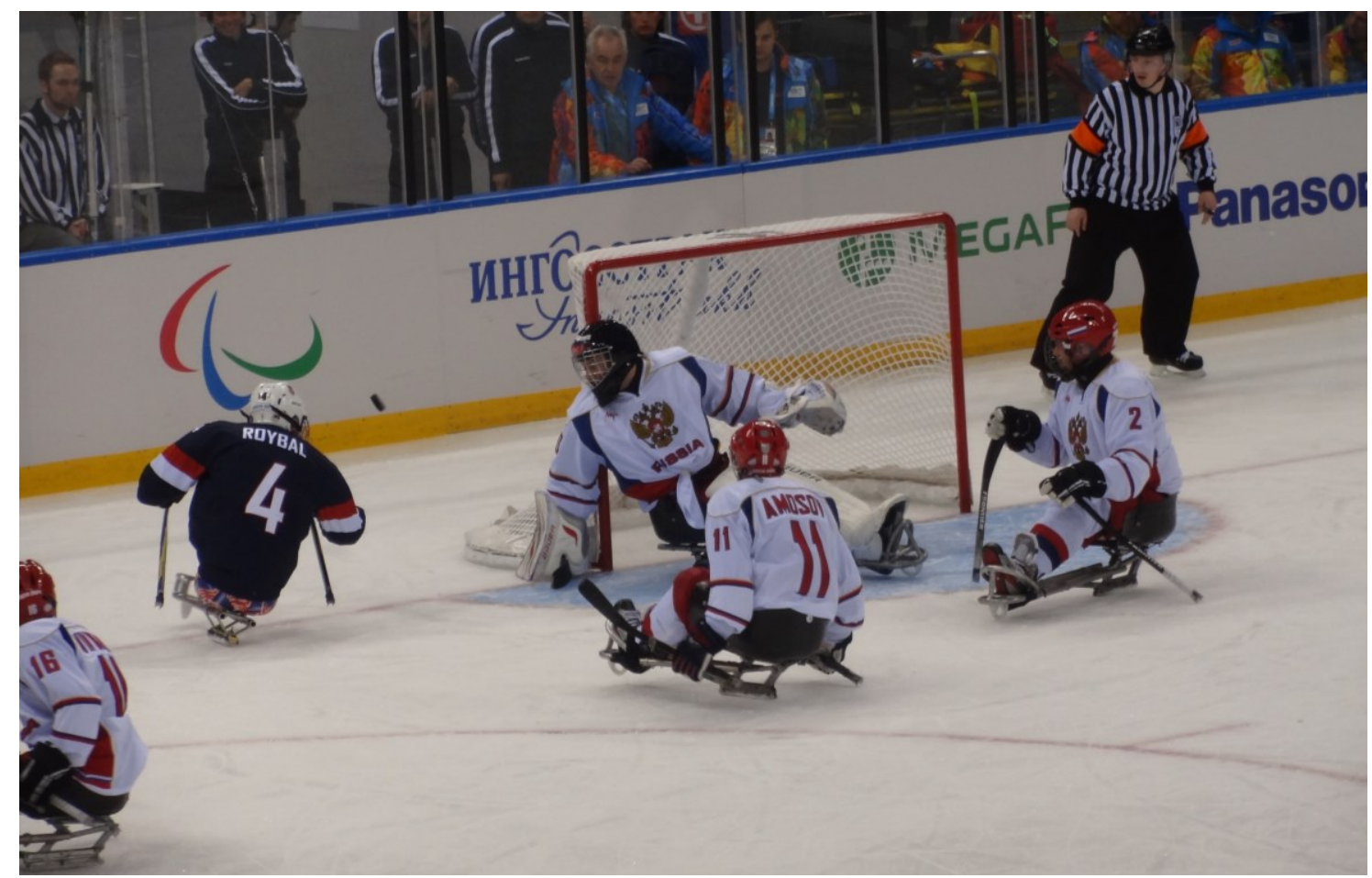

Figure 1.1 On-ice Paralympic sledge hockey depicting various phases of the stroking cycle and skating styles. Player No 2 is at rest. Player No 11 is at pick-off for his right arm, and in the recovery phase for his left (asymmetrical skating). Player No 4 is within the newly proposed preparation phase, and shows approximate bilateral symmetry (see Chapter 2). All players in motion (No 4, 11, 16) have flexed torsos [12].

Furthermore, sledge hockey is an increasingly popular para-sport where information from biomechanical analysis, specifically propulsion, can provide transferrable knowledge to enhance the understanding of the sport's most basic skillset, skating [9], [10]. Overall player performance may then be improved by determining what skating parameters need to be modified. This process can also be used to evaluate the potential for overuse and/or overloading onto/about the upper limbs.

Injury to the shoulder may arise because the architecture of the glenohumeral joint $(G H J)$ does not support structural competence for withstanding intense continuous weight-bearing 
forces [7], [13], [14]. Simple mechanics states that as an object's mobility increases, its structural integrity decreases [15]-[17]. The $G H J$ promotes the largest range of motion within the human body, which can result in decreased structural integrity, especially when tasked to perform cyclical weight-bearing poling motions. Webborn et al. reviewed injury patterns during the 2002 Winter Paralympic Games finding that sledge hockey had the highest rate of injury (14\%) per 100 athletes, and the second highest acquired injuries (31\%) of the 416 para-athletes competing [18]. Researchers implied injuries in sledge hockey may be unique and predictable based upon the sporting discipline adaptions to the para-athlete (e.g., equipment) [18]. Mechanisms of injury were identified as $26 \%$ traumatic and $5 \%$ overuse, with $50 \%$ of all injuries affecting the upper limbs of competing athletes (percentages are from all reported para-athlete injuries, i.e., sledge hockey, alpine sit-ski, and cross-country sit-ski) [18]. Hawkeswood et al. determined that the shoulder was injured $90 \%(n=10)$ of the time in sledge hockey, with $80 \%$ injury to the elbow, and $90 \%$ to the wrists [19]. Shoulder injuries were $100 \%$ sprains and the highest ranked injury within the sledge hockey injury and safety focused pilot study [19].

Aggregation of the above information implies that the onset of long-term injury involving muscular and/or connective tissues, and/or joint soreness is probable within the shoulder dependent population. Immobility, resulting from injury, could drastically limit a shoulderdependent individual, especially as a sledge hockey player. As the potential for injury is high, focused investigation of the forces generated during poling within the sport of sledge hockey is warranted. Although sledge hockey is a full body contact para-sport, discussion of the potential injury mechanisms caused by such impacts does not occur within this 
thesis.

As previously mentioned, the sport of sledge hockey has become increasingly popular, especially at the elite level [9], [10]. The need to establish a biomechanical viewpoint involving the sport of sledge hockey has emerged. Biomechanical investigations specific to this sport and its skating motion can provide various levels of information. This research scope can: 1) contribute to the minimally explored sledge hockey database improving sport-specific knowledge, and 2) heighten understanding regarding overuse/overloading of the upper limbs during seated bodyweight motion.

Specifically, this thesis will provide information regarding the mechanical system (upper limb and stick) at its point of rotation (shoulder), the respective reaction forces each limb system produces during contact, and how these forces may affect the shoulders.

\subsection{Objectives}

The objective of this thesis is to investigate propulsion and the respective reaction forces of the downward poling motion within the sport of sledge hockey. Specifically, this thesis includes: 1) a free fall solid-static limb system mechanical model, and 2) human participants using an off-ice sledge.

Properly designed mechanical models provide the opportunity for accurate, repeatable, systematic investigations. Specifically, within this thesis, the development and validation of a mechanical model is used to observe initial impact reaction forces from a free fall 
downward pole in sledge hockey. The ability to independently modify the mechanical model's joints (torso, shoulder, elbow, wrist-stick) allows for a systematic investigation of initial impact forces. Study 1 in this thesis investigates free fall downward poling of a mechanical model designed to replicate a male sledge hockey player $(S L A M-80)$. SLAM -80 is a solid wood prototype, with soft weights placed to mimic an average mesomorph male's arm holding a sledge hockey stick. SLAM - 80 stands for sledge (SL) $\operatorname{arm}(A)$ male $(M) 80 \mathrm{~kg}(80) . S L A M-80$ is fastened to the right side of a sledge at a $40^{\circ}$ torso flexion with the shoulder as the only dynamic joint (sagittal plane motion). SLAM 80 has a static elbow measuring either $120^{\circ}, 135^{\circ}$, or $150^{\circ}$, and an internal wrist-stick angle of $45^{\circ}$. The structural design for $S L A M-80$ is guided by military personnel data and body segment standard measures, whereas sledge hockey specific positioning is provided by piloted sledge hockey investigations and published researches from both alpine and cross-country sit-skiing [8], [20]-[25].

Comparing the mechanical model's results with human participant results provides validation of the mechanical model, and reliability of the investigated task (downward pole to impact). Human participant data are collected and presented to provide information for future iterations of the mechanical model, and future experimental design involving human participants and the sport of sledge hockey.

This thesis introduces indoor modifications to an on-ice sledge, which is used across an experiment specific 4-force plate design. A systematic approach created by restricting the wrist and elbow angles is also implemented during human participant tests that mimic the 
mechanical model. These restrictions create a static limb, which inhibits major movement from the above-mentioned joints, and makes the shoulder the sole point for motion production during task performance of the upper limbs. Isolation of the investigative joint (shoulder) reduces the number of assumptions required to model the movement. Such simplifications do limit how representative the research results are (i.e., how the system reflects actual sledge hockey biomechanics).

A dynamic limb is a complex biomechanical system involving numerous joints within the musculoskeletal system, each with different mechanical properties and contributions. In order to gain knowledge regarding this intrinsically dynamic task (kinetic energy involving the shoulder caused by downward poling impact), the additional dynamic components (limb and stick) are first observed as a static component (one-piece) within the system, with sequential distal dynamic parameters to be introduced in future research.

\subsection{Contributions}

The main contributions of this thesis are:

1. Determined baseline measures of the kinetic energy involved with the shoulder caused from impact in a free fall seated downward pole for the sport of sledge hockey.

Baseline measures provide primary evidence evaluating functionality and can be used to determine and/or measure deviations from this evaluated norm. Data acquired from SLAM - 80 involving free fall downward poling (limb-stick potential energy) provides baseline impact forces for skating (non-contractile motion), as outlined in 
Chapter 3. Results from $S L A M-80$ indicate that an average male sledge hockey player can produce similar joint-loading stresses $(2.0 \times B W$ and $2.3 \times$ $B W$, where $B W=$ bodyweight, mean and peak resultant impact force respectively) to the upper limb during a free fall downward pole, as compared to the stresses seen against the lower limb in running and going up stairs $(2.5 \times B W)$, or standing up $(2.0 \times B W)$ [26], [27]. From this, in conjunction with the cyclical pattern of skating, analogous overuse and/or overloading lower limb injuries can be predicted to potentially occur in the upper limb load-bearing joints, specifically concerning the point of rotation (shoulder) [26], [27].

Furthermore, a free fall downward stroke from the shoulders can produce greater locomotive force than other shoulder weight-bearing motions such as wheelchair propulsion $(0.1 \times B W)$ and rowing $(1.1 \times B W)[28]-[30]$. The respective peak specific power, which can be generated from a free fall downward pole per bodyweight $(-38 \mathrm{~W} / \mathrm{kg}$ limb extension) is considerably more than peak specific power per bodyweight produced from elite cyclists during cross-country off road races $(7 \mathrm{~W} / \mathrm{kg})$ [31]. From the above-mentioned information, this thesis interprets that baseline efforts to initiate skating in sledge hockey are more closely related to efforts associated with lower limb activities. Similar potential for injury to the upper limbs are predicted to be observed from skating in sledge hockey as compared to injuries caused by bodyweight lower limb activities. 
During free fall downward poling a slight difference exists between all tested elbow angles and peak horizontal force ( $A P$, anterior-posterior). Specifically, a flexed elbow produces less horizontal force than an extended elbow, which produced less horizontal force than a mid elbow $\left(R_{A P 120^{\circ}}<R_{A P 150^{\circ}}<R_{A P 135^{\circ}}\right.$, where $R$ is impact reaction force). No difference is observed between elbow angles and peak vertical force (Long, longitudinal). To heighten the validity of $S L A M-80$, an inverse dynamic mathematical model is developed and used to estimate the respective kinetic reactions involving the shoulder. Derived from peak resultant impact force, an extended elbow produces the least increase in shoulder joint reaction force $\left(J R F_{\text {Shoulder }}\right)$ as a result of stick impact. Results also suggest extending the elbow is slightly more efficient $(0.02 \mathrm{~N} / \mathrm{W})$ when producing propulsive (horizontal) forces during the downward pole (limb extension) compared to a flexed elbow. Moreover, the ability to extend the elbow allows for a more horizontal pick-plant improving force transfer producing locomotion. From these results, a flexed elbow can be viewed as a less than optimal position for skating in sledge hockey.

Absorption properties within the mechanical model are suggested as a potential limitation to the overall rigidity. From the above-mentioned results, the mechanical design of $S L A M-80$ has been validated. Data produced from $S L A M-80$ are compared against human participants in order to provide validation of the design, and reliability to the downward pole in sledge hockey (Chapter 5). The two top ranked participants (tested population ranked skill level) can achieve the SLAM - 80 baseline (peak resultant impact force $>2.0 \times B W$ ). Their propulsive force is less than 
$S L A M-80$ as a direct effect of increase stick angle. By validating $S L A M-80$, further research can occur with regards to the poling impact in sledge hockey while eliminating additional stresses onto a shoulder dependent individual.

Portions of this contribution have been disseminated in the following publications:

- A.M. Gal, A.D.C. Chan \& D. Hay, "Validating a solid-static single-armed male prototype tasked to produce dynamic movement from the shoulder through the preparation phase", in International Federation for Medical and Biological Engineering (IFMBE) Proceedings, World Congress on Medical Physics and Biomedical Engineering (IUPESM), Toronto, Canada, 2015, vol. 51, pp. 11461149. doi:10.1007/978-3-319-19387-8_278 [32].

- A.M. Gal, A.D.C. Chan \& D.C. Hay, "Investigating the seated double poling: Identifying baseline measures of the preparation phase", in 33rd International Conference on Biomechanics in Sport (ISBS), Poitiers, France, 2015, pp. 1-4. sciencesconf.org:isbs2015:59230 [33].

\section{Adapted on-ice sledge hockey equipment to be used off-ice within an indoor} MOCAP laboratory using a modified force plate configuration specific to seated double poling data collection.

Due to its infancy within the elite sporting world, sledge hockey lacks advanced technology for sport-specific off-ice training and experimental evaluation. Biomechanical computer models are typically based upon predetermined measurements of validated sport-specific baselines and/or standardized 
anthropometrics. Converting on-ice sledge hockey equipment to be used in a conventional motion capture (MOCAP) laboratory enabled data collection for preliminary sledge hockey skating motion analysis. Information obtained from these data can assist in establishing a sledge hockey database. A valid and reliable database could be used to create computer-simulated models to provide accurate training information.

All equipment is identified in Section 4.4. Modifications to equipment are transferrable to off-ice training, comparable to the relationship between roller/inline hockey and onice hockey, or roller-skiing and cross-country skiing.

\section{Determined peak force production and the derived kinetics during the contact} phase of the seated poling cycle in the sport of sledge hockey from task naïve participants.

Previous investigations of double poling cycles have suggested two peak forces exist during the propulsion phase (contact phase): 1) impact, and 2) push-off force. These two forces can be thought to be analogous to heel-strike and toe-off during hip gait analysis [24], [34], [35]. By investigating these forces within the seated poling cycle, parameters concerning shoulder gait analysis are developed (Chapter 6). Contributions of these forces onto the system are further investigated through impulse and rate of force development, as outlined in Chapter 3 and Chapter 4. 
Results from Study 2 indicate that naïve sledge hockey players create between 1.2 and $3.1 \times B W$ of resultant ground reaction forces $(G R F s)$ per arm in order to initiate skating in sledge hockey off-ice. At push-off, forces produced from each limb range between 0.3 and $0.4 \times B W$. From this, it can be predicted that potential injury is more likely to be caused from initial impact than at push-off for a sledge hockey player.

Impact potential injuries are predicted to be similar to injuries observed from repetitive quick and forceful motions onto load-bearing joints such as jumping [36], [37]. Propulsion phase potential injuries are predicted to be similar to other bodyweight motions such as sitting down and standing up, walking, and climbing stairs [27]. Pushoff potential injuries are predicted to be similar to overuse injuries observed in shoulder dependent populations caused from wheelchair propulsion [29], [30], [38]. Rehabilitative and preventative practices are suggested to focus concerns to enhance shoulder joint structural competence at impact from quick forceful poling motions. Definite effect(s) of these impact stresses are still unknown.

Increased stresses of these magnitudes from repetitive and continuous exposure foreshadow a more rapid onset of chronic overuse shoulder injuries commonly seen in the hips of distance runners [5], [6], [26], [27]. Overloading from the elevated impact forces may cause acutely related issues specifically at the load-bearing joints, and are predicted to promote chronic injuries involving the load-bearing limbs [6], [26], [27], [36], [37]. Additionally, since skating is a bilateral motion, both symmetrical and asymmetrical, overuse injuries to the load-bearing limbs resulting from side-to-side 
differences are also predicted to occur, a phenomenon observed in the lower limbs of distance runners [26].

From these interpretations, this thesis provides the shoulder-dependent population, a subpopulation of $P w D s$, with information to improve their quality of life and potentially minimize additional effects encountered during $A D L s$. Exercise prescriptions can be prescribed reducing and/or preventing overuse and overloading injuries to the load-bearing limbs, specifically for skating in sledge hockey.

Portions of this contribution have been disseminated in the following publications:

- A.M. Gal, D.C. Hay, \& A.D.C. Chan, "2 and 3-dimensional biomechanical analysis of the linear stroking cycle in the sport of sledge hockey: Glenohumeral joint kinematic, kinetic and surface EMG muscle modeling on and off ice", in Proceedings of 13th International Symposium on 3D Analysis of Human Movement (3D AHM), Lausanne, Switzerland, 2014, pp. 108-111. ISBN 9782880748562 [23].

\subsection{Overview}

The remainder of this thesis is organized as follows. Chapter 2 contains a literature review of the development of biomechanical analyses for dynamic movement using a MOCAP system in conjunction with surface electromyography $(s E M G)$ and force plates. A global co-ordinate system is introduced allowing algorithms to be established for measurements used in this thesis. The limited body of sledge hockey research related to this thesis is 
presented.

Chapter 3 presents Study 1: the development and analysis of $S L A M-80$. SLAM -80 is a 2-dimensional mechanical model designed to produce a free fall downward pole in the sport of sledge hockey. $S L A M-80$ is an anatomically correct solid-static average male upper limb system, which produces baseline reaction forces involving the shoulder at initial impact. Results from impact for various drop heights and elbow angles are presented throughout the chapter. Results are used to assist in the evaluation of similar musculoskeletal movements of human participants discussed in subsequent chapters.

Chapter 4 presents Study 2: task naïve off-ice skating in the sport of sledge hockey. Participant athletic abilities in relation to skating, participant preparation and procedures, data acquisition equipment, data processing methodologies, and results are discussed. Within a MOCAP laboratory, participants propel themselves in a modified indoor sledge across a 4-force plate system. To measure muscle activation, four bilateral posterior and one bilateral anterior wireless $S E M G$ electrodes (upper torso and upper limbs) are affixed to each participant. Participants' perform static start and mid cycle strokes across the force plates with video recording focusing on right arm force plate contact. Elbow and wrist restrictions are used to restrict range of motion to the upper limbs during specific tests. Raw data are processed and published via MATLAB script. Results from impact and pushoff are presented throughout the chapter. Results are used to assist in the evaluation of skating in the sport of sledge hockey. 
Chapter 5 presents the aggregated summary and discussion of Study 1: SLAM - 80, and Study 2: naïve sledge hockey participants.

Chapter 6 presents sport-specific and generalized shoulder-dependent population conclusions and interpretations based on the results and discussions of this thesis.

Chapter 7 provides future research, limitations, and amendments for this thesis. 


\section{Chapter: Background \& Literature Review}

This chapter consists of four sections. Section 2.1 describes the sport of sledge hockey, providing definitions for skating and stroke cycle phases, derived from analogous literature regarding the biomechanics of cross-country skiing and hip gait analysis. Sledge hockey specific literature provides information with respect to game performance, physiological game efforts, equipment, and injury.

Section 2.2 outlines exploratory knowledge regarding biomechanical analysis of the musculoskeletal system through the use of 3-dimensional MOCAP and $s E M G$. Literature supports evaluation techniques used to investigate the stroking cycle within the sport of sledge hockey. Throughout this section, the definition for the upper arm as a dynamic mechanical system is developed with the focal point of rotation about the shoulder. Literature outlines the complexity of estimating in-vivo forces identifying limitations during analytical procedures.

Section 2.3 provides specific information regarding solving a biomechanical investigation through the use of MOCAP, $S E M G$, and force plates. Specifics include signal acquisition, SEMG and MOCAP signal processing, sampling frequencies commonly used during biomechanical analysis, reasoning to apply a digital filter or not to the raw signal, and details on converting the signal into processed data.

Section 2.4 provides a summary and conclusion of information of the chapter with regards to skating in sledge hockey. 


\subsection{The Sport of Sledge Hockey}

In Sweden during the $1960 \mathrm{~s}$, at a rehabilitation centre, two physically disabled men established the sport of sledge hockey as a way to continue to play ice hockey [8], [39]. Sledge hockey is full body contact para-hockey played on-ice following similar rules to those of ice hockey [8], [39]. During game play, each team has six players on the ice, including a goaltender in the net [8], [39], [40]. Non goaltenders (forwards and defense) are equipped with two miniature sticks, with blades at one-end for handling the puck and picks at the other to produce movement (Figure 2.1) [8], [39], [40]. To date, sledge hockey is the most popular Paralympic sport [9]. Sledge hockey's unique equipment design allows both abled and physically disabled persons to participate.

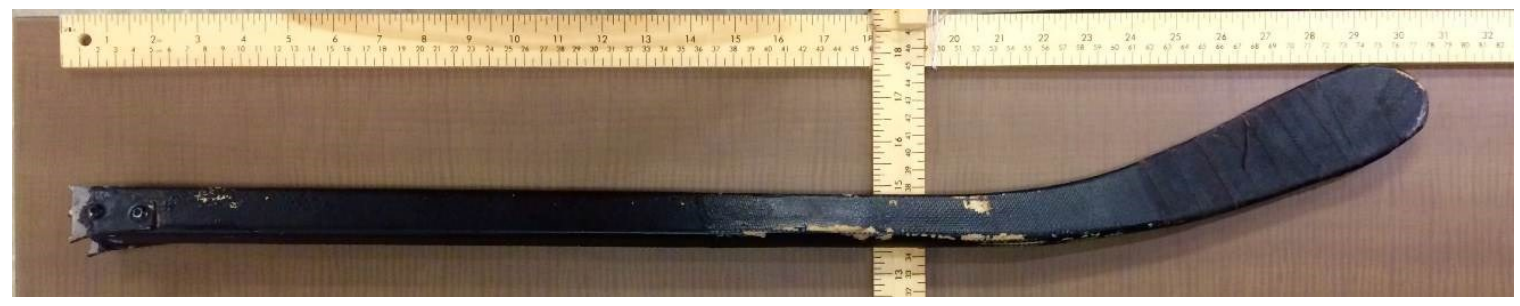

Figure 2.1 A typical right wooden sledge hockey stick with on-ice picks (to scale - picks start at $1.00 \mathrm{~cm})$.

Stick handling occurs as the puck slides under the leg-rests between the left and right stick blades, or on one side of the body with the respective stick blade and puck. Players are typically ambidextrous shooters. Players flip the sticks alternating between blades and picks to maintain puck control and motion. There are many variations for motion production within the sport of sledge hockey; most common, is a bilaterally symmetrical stroke cycle. 


\subsubsection{Skating in Sledge Hockey}

A forward cyclical shoulder-driven motion known as skating creates weight-bearing locomotion within the sport of sledge hockey. Skating resembles similar motions to those observed in cross-country skiing, particularly the double poling motion. Muscular force produced from the torso and each arm is transmitted along the respective stick to the picksurface interface, where muscular energy is transferred into forward propulsion as a result of the picks digging into the ice surface. Increased force during motion production implies a positive deviation (increase) in forward propulsive output (displacement, velocity, acceleration, etc.). Analogs to bipedal trajectories and standard biomechanical parameters observed in hip gait analysis, during normal upright walking and running, can be made for skating [35].

Previous research identifies propulsion (skating) to consist of three phases: 1) propulsion, 2) recovery, and 3) preparation [11]. From this research, the propulsion phase is defined as the contact phase between pick initial impact and push-off, analogous to the stance phase in hip gait analysis (heel-strike and toe-off, respectively) [11]. A contact-free phase or return of the cycle follows propulsion, analogous to the swing phase in hip gait analysis.

Lomond and Wiseman conducted a review involving the kinematics of the stroking cycle within the sport of sledge hockey focusing on injury prevention relating to wheelchair use [11]. Rehabilitation textbooks cite their work when discussing para-sport injuries and ADLS with overuse and chronic shoulder pain [41]. Lomond and Wiseman's results provide introductory guidelines to the phases of skating within the sport of sledge hockey, which they defined as propulsion [11]. 
Published results regarding the sledge hockey stroke cycle suggests the preparation phase exists from maximal shoulder hyperextension and maximal arm extension to the instant preceding initial pick contact [11]. This implies that recovery is the shortest phase, occurring from pick-off to the onset of the preparation phase [11]. Preliminary boundaries outlined for the recovery and preparation phases within sledge hockey contradict upright double poling and standard hip gait analyses, which suggests recovery to be the longest phase [11], [34], [35], [42]. Lomond and Wiseman's published results lack supporting documentation of how the phases within propulsion are determined, and from what form of evidence (e.g., visual observation, motion capture); they are implied to be derived analogous to wheelchair stroking cycles [11]. Sledge hockey phase cycle results are believed to exist in Lomond's unpublished Bachelor's Thesis entitled, "A Kinematic Analysis of Sledge Hockey Propulsion" [43]. Various attempts to retrieve this work were unsuccessfully made.

Since the foundation of the stroking cycle within the sport of sledge hockey displays biomechanical similarities with hip gait and upright double poling, this thesis redefines the location for the start of the preparation phase. Preparation, from a sagittal plane viewpoint, initiates at pick maximal vertical displacement during shoulder flexion (player specific) terminating at the instant preceding pick contact. The revised stroking cycle within the sport of sledge hockey (Figure 2.2) is analogous to the stroking cycle observed in upright double poling. Support for the shift in phase initiation strongly relates to the newly identified preparation phase in upright cross-country skiing. Future research is designed to investigate the exchange point where the recovery phase changes into the preparation phase 
(Figure 2.2 (a) and (d)); this boundary is unclear among all poling sports.

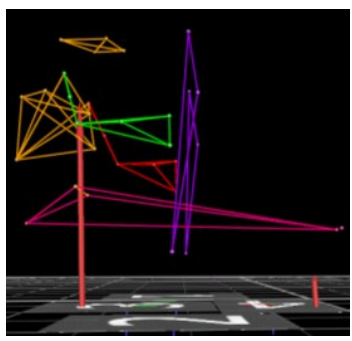

(a)

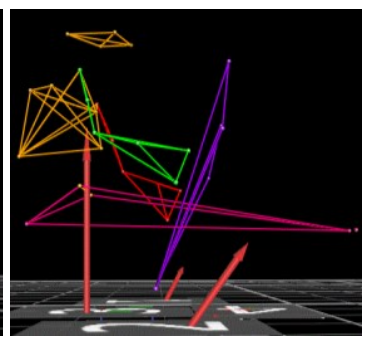

(b)

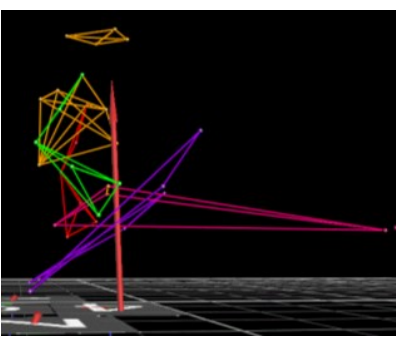

(c)

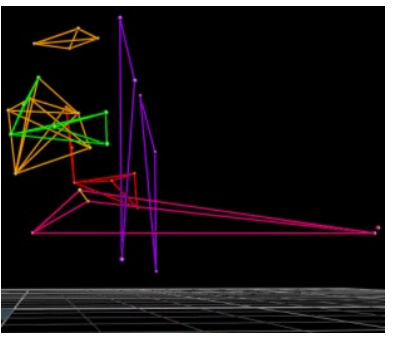

(d)

Figure 2.2 MOCAP computer regenerated single gait cycle showing phase boundaries for the sport of sledge hockey (a) Recovery termination - Preparation initiation (pick y max), (b) Propulsion impact (pick $y=0 \mathrm{~m}$ at $\left.t_{\text {Impact }}\right)$, (c) Propulsion Recoil - Recovery initiation $\left(y_{i+1}\right.$, where $y_{i}=0 \mathrm{~m}$ at $\left.t_{\text {Recoil }}\right)$, and (d) Recovery termination - Preparation initiation (pick $y$ max). Yellow - head and torso, pink Rib 10, lower body, and sledge, red - right limb, green - left limb, and purple - sticks.

Biomechanical trends for the upright preparation phase, which occurs prior to pole plant, are believed to be "linked to a high impulse of pole forces, appropriate timing of forces during the poling phase and an altered pattern during the swing phase with the poles being placed more vertically at pole plant, with more distance covered in a forward direction" $[34$, p. 403]. From this, biomechanical trends for the sport of sledge hockey are predicted to occur closer to initial contact opposed to the previously suggested post contact location. Parallel inferences from the biomedical trends outlined by Stoggl and Holmberg are used to assist in developing observable shoulder gait parameters for skating analysis in sledge hockey, which are presented in Chapter 7 [34]. This thesis seeks to evaluate the propulsion phase using human experimental and modelling data. 
Parallel evidence for the previous identified preparation and recovery phase locations in the sport of sledge hockey is provided from cross-country sit-ski biomechanical trends. Previous research suggest transition (preparation) to occur post contact to maximal arm extension [24], [44]. Sit-ski biomechanical trends are identified as a contact free anteriorposterior arm swing [24]. Research supporting cross-country sit-ski stroke analysis is as limited as sledge hockey skating investigations, with this thesis citing both publications regarding stroke analysis to date. Cross-country sit-ski pole lengths are significantly longer than sticks used in sledge hockey. The relationship for pole length and sport-specific acromial height correlates positively when comparing sledge hockey to upright skiing. This correlation provides additional support to reinvestigate the location for preparation initiation within the sport of sledge hockey.

Since an evidence-based definition of the preparation phase is lacking across all poling cycles, this thesis seeks to provide evidence supporting the redefined location of preparation initiation based upon the observations outlined by Stoggl and Holmberg, specifically impulse and pole plant angles [34]. As there is no research involving skating biomechanics in sledge hockey, evaluation of altered swing phase mechanics and appropriate timing cannot be addressed within this thesis.

Skating specific biomechanical produced motions are suggested to be a large range of motion at the shoulder during the switch from hyperextension to flexion, and a small range of motion at the elbow from maximal extension to flexion [11]. However, to the best of the author's knowledge, no peer-reviewed literature exists that investigates the biomechanics 
of sledge hockey propulsion (i.e., from maximal shoulder flexion to the end of pole contact). From this, a gap exists involving motion production for the sport of sledge hockey; specifically, the range outlined from Stoggl and Holmberg as a performanceadvancing phase [34]. Since skating displays a cyclical pattern, a large range of motion is expected during the return of the cycle, which is typically understood as recovery in gait cycle analysis, again in support of the change in location for preparation initiation within the poling cycle [35].

\subsubsection{Theoretical, Mechanical, and Anatomical Double Poling Locomotion}

By understanding the biomechanics of a movement, modifications to improve motion production and reduce injury risk may be possible. Evidence regarding contact interfaces (e.g., angle, rate, duration) and force production can prove vital to understanding overuse and/or overloading injuries. Theoretically, as a task improves, the body should move more fluently and decrease stresses onto the load-bearing joints. However, high-impact, highvelocity motions such as sledge hockey may produce increased stresses onto load-bearing joints as a result of improved skating skills (e.g., increased acceleration, velocity, force production).

Since there is limited information regarding the sport of sledge hockey, previous researches from other similar poling sports such as upright and seated cross-country skiing can provide guidelines for biomechanical investigations and interpretation of results. Mechanically, optimal forward displacement occurs through symmetrical bilateral and repetitive trajectories. Symmetrical bilateral push-off promotes elevated force output compared to 
alternating unilateral poling, which is used more for balance and turning within the sport of sledge hockey. Increased stroke speed and/or increased force production produces larger displacements. Pick impact and push-off angles also play crucial roles in obtaining optimal results. Optimal force transfer during poling is desired throughout propulsion. Mathematically, horizontal pole push-off angles produce the greatest forward force transfer. Mechanically, a combination of horizontal and vertical forces produce linear (1dimensional) forward locomotion. Anatomically, tri-axial forces exist throughout the cycle inviting theoretical and mechanical hypotheses to be tested.

As previously stated, there is limited research involving the sport of sledge hockey. Crosscountry skiing poling cycles have been biomechanically studied by various researchers as indicated in sub sequent sections. Both cross-country skiers and sledge hockey players can use a double poling stroke, providing a commonality and initial starting point for skating analysis in sledge hockey. Specifically, para-skiing, both alpine and cross-country, can provide insight towards the effect of sitting during the poling cycle, where sport-specific acromial height and respective pole lengths are more closely correlated between sledge hockey and upright cross-country skiing. Ideally, the start sprint in alpine sit-skiing correlates the closest to skating in sledge hockey (explosive quick bursts), however, to the best of the author's knowledge no biomechanical research currently exists. Poling cycles and pole contact angle range (initial, peak, and push-off) promoting enhanced performance during double poling in elite cross-country skiing are discussed below. 


\subsubsection{Cross-Country Sit-Skiing: Biomechanical Review}

Cross-country skiing has been extensively studied among various disciplines over the previous decades. Throughout these investigations, pole angle has been measured from both the horizontal and the vertical axes. All presented results have been manipulated to reflect this thesis' pick impact angle definition. For the purpose of this thesis all pick impact angle data with respect to the sagittal plane have been presented from the anterior-posterior axis $\left(0^{\circ}\right)$ counter clockwise to the longitudinal axis $\left(90^{\circ}\right)$, where $x, y>0$ and positive $x$ is the forward direction of travel.

Video analysis of cross-country sit-ski results from Torino's 2006 Paralympics 15 km racecourse indicated that, on average, group one (first through fifth place) produced flat ground impact angles for lap one $72^{\circ} \pm 3^{\circ}$ and lap four $75^{\circ} \pm 4^{\circ}$, and uphill poling impact angles for lap one $69^{\circ} \pm 4^{\circ}$ and lap four $69^{\circ} \pm 5^{\circ}$ [44]. Group two (last five placings) produced flat ground impact angles for lap one $64^{\circ} \pm 8^{\circ}$ and lap four $67^{\circ} \pm 5^{\circ}$, and uphill poling lap one $62^{\circ} \pm 5^{\circ}$ and lap four $66^{\circ} \pm 3^{\circ}$ [44]. Results indicated that the only significant difference $(p=0.003)$ existed between flat and uphill impact angles; no significant difference existed between groups [44].

A markerless stereophotogrammetric acquisition system was used to produce video data of the cross-country sit-ski sprint race qualifiers for Vancouver's 2010 Paralympics [24]. Results indicated that a sit-skier produced poling angles ranging from $79^{\circ}-12^{\circ}$, impact through to push-off [24]. Left-side sledge velocities peaked late in propulsion through to the transition phase $(<5.2 \mathrm{~m} / \mathrm{s})[24]$. As previously mentioned, cross-country sit-ski pole 
length is significantly longer than a sledge hockey stick, creating a distant relation to the biomechanics of the stroke cycle. Results from the cross-country sit-ski research are used more as a guideline than direct comparison, with specific focus regarding the relationship of the seated weight-bearing poling motion.

\subsubsection{Upright Cross-Country Skiing: Biomechanical Review}

Interpretation of a positive correlation regarding upper limb mechanics during poling between the sports of sledge hockey and upright cross-country skiing can be made from the direct relationship of pole length and sport-specific acromial height.

$M O C A P$ data and pole mounted strain gauge force transducers were used to acquire results from sixteen elite male upright skiers during incremental speed roller-ski treadmill sprint tests $\left(v_{i}=22.5 \mathrm{~km} / \mathrm{h}+1.5 \mathrm{~km} / \mathrm{h} / 10 \mathrm{~s}\right.$ until individual maximum, where $v_{i}=$ initial velocity of treadmill) [34]. Two peaking forces were evaluated per pole contact force curve: 1$)$ impact force ( $1^{\text {st }}$ peak on the resultant force curve), and 2) peak force (peak horizontal force post impact force) [34]. Impact resultant force occurred at initial pole contact producing $325 \mathrm{~N}$ for the fastest skier, and $345 \mathrm{~N}$ for the slowest [34]. The fastest skier then produced peak resultant force of $475 \mathrm{~N}$ around $0.08 \mathrm{~s}$ compared to the slowest skier peak pole force of $275 \mathrm{~N}$ around $0.06 \mathrm{~s}$ [34].

Resultant force curves for both skiers displayed similar trajectories with the exception of an absolute peak resultant force recorded around $0.02 \mathrm{~s}$ and just under $400 \mathrm{~N}$ from the slowest skier [34]. From the above-mentioned impact and peak force definitions, the 
absolute peak resultant force of the slowest skier occurred too late and too early, respectively, within the contact phase to be considered for force analysis [34]. This post impact spiking force was present in the fastest skier resultant force curve, however, the spiking force was not greater than the recorded impact force [34]. Horizontal force remained under $200 \mathrm{~N}$ for both skiers throughout the entirety of the force curves with peak values correlating to peak resultant forces $(r=0.86, p<0.001)$ [34]. Pole angles from impact to push-off ranged from approximately $83^{\circ}-25^{\circ}$ for the fastest skier, and $74^{\circ}-$ $16^{\circ}$ for the slowest [34].

Using the same subjects, another study, with focus on sprint skiing styles and muscular strength tests, determined that submaximal speed (mean of 10 cycles in the last $10 \mathrm{~s}$ of the increment all skiers completed) peak pole force was $334 \mathrm{~N} \pm 70 \mathrm{~N}$, whereas maximal speed (maximally reached speed increment, participant specific) peak pole force was $303 \mathrm{~N} \pm 68 \mathrm{~N}$ for the double poling stroke style [45]. Impulse was determined to be $38 \mathrm{Ns} \pm 7 \mathrm{Ns}$ for submaximal speeds, and $24 \mathrm{Ns} \pm 3 \mathrm{Ns}$ for maximal speeds [45]. A highly significant difference $(p<0.001)$ was determined to exist between submaximal and maximal speed impulses, where no significant difference existed between peak pole forces within submaximal and maximal speeds for the double poling stroke cycle [45].

Unpredicted greater submaximal pole force results were also observed in the V2 ski style, however, not in the diagonal ski style [45]. Technical observations were made regarding the change in skiing style as faster skiers approached maximal speeds; specifically, the addition of a double-push to the V2 ski style [45]. Two different poling strategies were 
observed during the double poling ski style, where one strategy produced longer poling times $(p<0.001)$ and longer time-to-peak $(p<0.01)$ at submaximal speeds [45]. Authors implied that faster skiers made a strategic performance choice at maximal speeds resulting in a lower force output and lower impulse with an increase in cadence (strokes per minute) [45]. From this, the work duty cycle of the faster skier at maximal speed was increased [45]. Work duty cycles in biomechanics involve work produced over the combination of work produced and rest taken [46]. Work duty cycles have direct effects on muscle fatigue in turn affecting potential for work related injuries [46] .

In accordance with other researches involving cyclical characteristics throughout various speeds, time to produce contact poling was inversely proportional to overall speed for both double poling and V2 ski style [45], [47]-[49]. Slower skiers displayed the second strategy and produced peak force at impact compared to late contact phase as observed in faster skiers [45]. As a result, faster skiers produced increased propulsive force at more horizontal pole angles occurring later in the contact phase; optimal force generation location was still unclear from these results [45].

Interpretation of an increase in force over shortened duration suggests maximal speeds potentially introduce increased stresses involving the joints compared to submaximal speeds. Changes in technique as maximal speeds are reached may reduce improper poling, in turn decreasing impact forces onto the load-bearing limbs. Improvement in poling may decrease the potential for injury from the elevated stresses introduced at/about the joints. Improved stroke mechanics and proper timing for propulsive force generation should be 
considered key factors for injury reduction and/or prevention during poling sports.

Seven elite male cross-country skiers participated in a laboratory downhill roller-ski pole plant test, which compared various pole lengths $( \pm 7.5 \mathrm{~cm}$ from personal pole length categorized as short, normal, and long) and the respective contact reaction forces (single left force plate) [50]. Mean horizontal (propulsive push motion) impact force for short poles was $109 \mathrm{~N} \pm 15 \mathrm{~N}$, normal $105 \mathrm{~N} \pm 15 \mathrm{~N}$, and long $102 \mathrm{~N} \pm 12 \mathrm{~N}$ [50]. Vertical (downward motion) impulse was $46 \mathrm{Ns} \pm 5 \mathrm{Ns}$ for short poles, $51 \mathrm{Ns} \pm 5 \mathrm{Ns}$ normal poles, and $54 \mathrm{Ns} \pm 7 \mathrm{Ns}$ long poles [50]. Horizontal impulse was $44 \mathrm{Ns} \pm 4$ Ns for short poles, $47 \mathrm{Ns} \pm 5 \mathrm{Ns}$ normal, and $48 \mathrm{Ns} \pm 5 \mathrm{Ns}$ long [50]. Impulse and horizontal impact force for short and normal pole lengths were significantly different $(p<0.05)$ to the next longer pole length [50].

Sagittal plane impact angles were $77^{\circ}, 81^{\circ}$, and $84^{\circ}$, with peak horizontal force occurring at $42^{\circ}, 43^{\circ}$, and $45^{\circ}$, and push-off angles approximately $22^{\circ}, 21^{\circ}$, and $21^{\circ}$, short, normal, and long poles, respectively [50]. Pole impact angle was determined to be significantly different based upon the previous mentioned methodology [50]. Duration of the thrust phase was $0.41 \mathrm{~s} \pm 0.02 \mathrm{~s}$ short poles, $0.44 \mathrm{~s} \pm 0.02 \mathrm{~s}$ normal, and $0.47 \mathrm{~s} \pm 0.03 \mathrm{~s}$ long [50]. Short and normal pole length thrust durations were determined to be significantly different [50].

From the above information, a potential mechanism of injury for poling sport athletes could be improper pole length. Dependent upon interpretation of results (maximal force 
generation or maximal momentum), both a shortened and lengthened stick could potentially introduce added stresses during performance output. A standardized stick length within the sport of sledge hockey may not prove to be adequate for all players. Potential future research could investigate stick length using similar methods to the above study in order to determine if variation of stick size should be implemented to the current sledge hockey stick design.

Eleven elite cross-country skiers participated in an incremental maximal velocity roller-ski treadmill test (similar methodology as above) with focus on the double poling cycles as a whole (trajectories, reaction force, and muscular contributions) [42]. Right pole force was acquired through an experiment-specific adjustable carbon-fibre pole equipped with a strain gauge force transducer [42]. Peak impact force is defined by the respective peak resultant impact GRFs. Peak impact force was $235 \mathrm{~N} \pm 63 \mathrm{~N}$ with an impulse of $36 \mathrm{Ns} \pm$ $6 \mathrm{Ns}$, and a time to peak force of $0.10 \mathrm{~s} \pm 0.02 \mathrm{~s}$ [42]. Kinematics were measured with goniometers and video analysis specifically involving the elbow, hip, and knee [42].

Similarly, twelve elite cross-country skiers participated in a five-stage incremental velocity maximal roller-ski treadmill test with focus on various double poling velocities [51]. Again, kinematics were acquired by goniometers and processed for investigation of elbow, hip, and knee angles [51]. Peak impact force was determined to be $250 \mathrm{~N} \pm 71 \mathrm{~N}$ with a rate of force development of $3038 \mathrm{~N} / \mathrm{s} \pm 956 \mathrm{~N} / \mathrm{s}$, and a time to peak force of $0.08 \mathrm{~s} \pm$ $0.01 \mathrm{~s}$ at $27 \mathrm{~km} / \mathrm{h}$ treadmill velocity, the stage prior to velocity maximum [51]. Peak impact force for the initial stage, $9 \mathrm{~km} / \mathrm{h}$ treadmill velocity, was determined to be the 
lowest peak force at $145 \mathrm{~N} \pm 82 \mathrm{~N}$ with a rate of force development of $804 \mathrm{~N} / \mathrm{s} \pm$ $869 \mathrm{~N} / \mathrm{s}$, and a time to peak force of $0.21 \mathrm{~s} \pm 0.04 \mathrm{~s}$ [51]. Peak impact force, rate of force production, and time to peak force were determined to be significantly different $(p<0.05)$ for the first two stages (second stage was $15 \mathrm{~km} / \mathrm{h}$ treadmill velocity) to the remaining respective stages [51]. The velocity maximum stage was significantly different $(p<0.05)$ only to the first two stages [51]. The stage prior to maximum $(27 \mathrm{~km} / \mathrm{h})$ was determined to be significantly different $(p<0.05)$ to all preceding stages (middle stage was $21 \mathrm{~km} / \mathrm{h}$ treadmill velocity) [51].

Finally, twelve elite male cross-country skiers participated in a ski-tunnel artificial snow sprint course with a focus on start, race, and finish sprinting forces [52]. Forces were acquired from a custom-designed $20 \mathrm{~m}$ long force plate system [52]. Four heats were recorded with a 20-minute participant specific recovery in between, similar to protocols for official sprint heat competitions [52]. Results for vertical measures during heat one start sprint were peak force $383 \mathrm{~N} \pm 69 \mathrm{~N}$, impulse $38 \mathrm{Ns} \pm 6 \mathrm{Ns}$, and duration to peak force $67 \mathrm{~ms} \pm 11 \mathrm{~ms}$ [52]. Results for vertical measures during the final heat finish sprint were $302 \mathrm{~N} \pm 54 \mathrm{~N}, 34 \mathrm{Ns} \pm 5 \mathrm{Ns}$, and $80 \mathrm{~ms} \pm 18 \mathrm{~ms}$ [52]. Results for horizontal measures during heat one start sprint were peak force $195 \mathrm{~N} \pm 45 \mathrm{~N}$, impulse $24 \mathrm{Ns} \pm 4 \mathrm{Ns}$, and duration to peak force $88 \mathrm{~ms} \pm 16 \mathrm{~ms}$ [52]. Results for horizontal measures during the final heat finish sprint were $145 \mathrm{~N} \pm 30 \mathrm{~N}, 22 \mathrm{Ns} \pm 3 \mathrm{Ns}$, and $99 \mathrm{~ms} \pm 20 \mathrm{~ms}$ [52]. Each race and finish sprint vertical peak force per heat were significantly different $(p<0.05)$ from their respective start sprint, where only each race sprint horizontal peak force per heat were significantly different $(p<0.05)$ to the respective start sprint [52]. Consistency 
within the heats was observed for peak force depletion (start force $>$ race force $>$ finish force) for both vertical and horizontal forces throughout their respective heats [52].

The above-mentioned investigations provide baseline data for comparison with the two studies conducted within this thesis. Table 2.1 provides aggregated results of the abovementioned studies. By transferring this information towards skating in sledge hockey, elevated forces can be predicted to occur during pick contact. If an increase in impact force is observed during propulsion in sledge hockey, it would suggest skating is mechanically more forceful than skiing, and can also be a direct link to heightened risk for upper limb injury. Elite para-ski athletes reported injury to the upper limb 1.4 times more often than any other injury, with frequencies of chronic (30\%) and acute (25\%) injuries being relatively similar [53]. As skating in sledge hockey is a cyclical motion, overuse injuries are also predicted to be caused by the continuous use of the load-bearing limbs. 
Table 2.1 Cross-country skiing double poling kinematic and kinetic data

\begin{tabular}{|c|c|c|c|c|c|c|c|c|c|}
\hline & \multicolumn{3}{|c|}{ Peak Force (N) } & \multicolumn{3}{|c|}{ Peak Impulse (Ns) } & \multicolumn{3}{|c|}{ Impact Angle ( ${ }^{\circ}$ ) } \\
\hline & Resultant & Long & $\boldsymbol{A P}$ & Resultant & Long & $A P$ & Initial & Peak & $\begin{array}{l}\text { Push- } \\
\text { off }\end{array}$ \\
\hline \multicolumn{10}{|c|}{ UPRIGHT DOUBLE POLING } \\
\hline $\begin{array}{l}\text { Holmberg et } \\
\text { al. }[42]\end{array}$ & $\begin{array}{l}235 \pm \\
63\end{array}$ & & & $\begin{array}{l}36 \pm \\
6\end{array}$ & & & & & \\
\hline $\begin{array}{l}\text { Lindinger } e t \\
\text { al. [51] } \\
\text { Lindinger \& }\end{array}$ & $\begin{array}{l}250 \pm \\
72\end{array}$ & & & & & & & & \\
\hline $\begin{array}{l}\text { Holmberg } \\
{[54]}\end{array}$ & $\sim 450$ & & & & $\sim 48$ & & & & \\
\hline $\begin{array}{l}\text { Mikkola et } \\
\text { al. [52] }\end{array}$ & & $\begin{array}{l}397 \pm \\
77\end{array}$ & $\begin{array}{l}197 \pm \\
48\end{array}$ & & $\begin{array}{l}39 \pm \\
7\end{array}$ & $\begin{array}{l}25 \pm \\
4\end{array}$ & & & \\
\hline $\begin{array}{l}\text { Nilsson et } \\
\text { al. [50] }\end{array}$ & $\begin{array}{l}157 \pm \\
16\end{array}$ & $\begin{array}{l}113 \pm \\
12\end{array}$ & $\begin{array}{l}109 \pm \\
15\end{array}$ & & $\begin{array}{l}54 \pm \\
7\end{array}$ & $\begin{array}{l}48 \pm \\
5\end{array}$ & $77-84$ & $42-45$ & $21-22$ \\
\hline $\begin{array}{l}\text { Stoggl et al. } \\
{[45]}\end{array}$ & $\begin{array}{l}334 \pm \\
70\end{array}$ & & & $38 \pm 7$ & & & & & \\
\hline Stoggl \& & & & & & & & & & \\
\hline $\begin{array}{l}\text { Holmberg } \\
\text { [34] }\end{array}$ & $\sim 475$ & & $<200$ & & & & $\sim 83$ & & $\sim 16$ \\
\hline \multicolumn{10}{|c|}{ SEATED DOUBLE POLING - LONG POLE } \\
\hline $\begin{array}{l}\text { Bernardi et } \\
\text { al. [44] }\end{array}$ & & & & & & & & $57-79$ & \\
\hline $\begin{array}{l}\text { Gastaldi et } \\
\text { al. [24] }\end{array}$ & & & & & & & $\sim 79$ & & $\sim 12$ \\
\hline
\end{tabular}

\subsubsection{Existing Research on the Sport of Sledge Hockey}

Partly due to its infancy within the elite sporting world, research involving sledge hockey is scarce, creating gaps between the requirement to produce optimal performance and the knowledge of how to achieve it. As this high-velocity, high-impact sport has become a major winter Paralympics event, greater scientific analysis is warranted. Video analysis of previously recorded elite level sledge hockey games sets the precedence for providing sport-specific information [55]-[58].

Beckman et al. developed an initial checklist of observable player skills classified as offensive (seven skills) or defensive (three skills) [57]. Crudely, four categories of 
mathematically manipulated sub-scores were developed and used to rank players via videoanalysis [57]. Sub-score overviews included: 1) skating with the puck and received body checks, 2) passes and successful passes, 3) shooting, and 4) body checks and stick checks [57]. Utilizing this protocol, Kudlacek et al. analyzed the eight teams participating in Torino's 2006 Winter Paralympics ranking seventy-three players (minimum seven and maximum thirteen players per team) [56], [57]. Player skill levels were evaluated, and their overall ranking compared to their respective team's final 2006 Paralympic standing [56]. Major differences existed between both player and team rankings for the foundational skillset of skating with the puck, with players of elite skill displaying obvious increased task delivery [56]. Suggested improvements were directed towards the introductory player with focus on development of foundational skillsets such as skating and puck control [56]. Limitations for both studies included reliability of the scoring and ranking systems due to the infancy of the protocol [56], [57].

Hayrinen et al. incorporated this ranking system in conjunction with other prominent invasion style sport researches creating a modified ranking scheme for match analysis [55], [57]. A total of seven categories were created as observable skills and used for match analysis involving twenty-three goals scored within Vancouver's 2010 Paralympics (penalty shots, empty netters, and overtime goals were excluded) [55]. Positional ranking (offense vs. defense), winning vs. losing teams, and top four vs. bottom four placed teams were analyzed from post round-robin games (eight games) gaining an in depth understanding of the skills utilized to create elite level sledge hockey [55]. Similarly, results indicated that higher-ranking teams demonstrated elevated skill levels and game 
control [55].

Conventional laboratory-based research methods focused concerns towards injury prevention and physiological effects against performances providing preparation and training improvements for the Paralympian [19], [58]-[61]. Molik et al. observed no significant difference in game performance between four groups of physical disability categories [58]. However, a significant difference $(p<0.05)$ was determined to exist between the groups and training frequencies, with the 'other group' doubling training hours per week compared to the remaining three groups (double amputee, single amputee, and spinal cord injuries) [58]. From these results, classifying sledge hockey players may be unnecessary; however, a classification system may benefit lower functioning $P w D s$ belonging to a less homogenous group [58].

Hawkeswood et al. determined that upper limb prevention and protection was required in sledge hockey, specifically prevention of overuse injuries introduced from the mechanics of the stroking styles [19]. From Section 1.1, shoulder injuries were 100\% sprains and the highest ranked injury within this pilot study [19]. Hawkeswood et al. suggested that during the propulsion phase, a higher demand is placed on the upper limb joints and musculature as a result of the distinct pulling motion [19]. From this, a heightened understanding of propulsion phase biomechanics can be identified as an essential means to provide transferrable preventative information. 
Various researches have been conducted investigating the effects of upper body strength training against sprint kinetic outputs [59]-[61]. Results unanimously concluded that upper body strength was correlated with sprint speed and endurance, suggesting upper body focused training sessions promote maximal anaerobic strength and endurance [59]-[61]. Understanding stroke mechanics with optimal power output was also unanimously suggested as a significant research demand [59]-[61].

Worden-Rogers concluded that a $140^{\circ}$ knee angle with a medium knuckle height $(0.165 \mathrm{~m}$ seat height from ice) produced significantly $(p<0.05)$ faster test times [62]. Moreover, every participant acquired a faster time in one or both of the sprint and agility tests using the provided sledge compared to their personal sledges, indicating that players can improve current sitting positions within their own sledges [62]. Limitations were small sample size and lengthy evaluation completion timelines [62].

The previous research does not represent all investigations involving sledge hockey; however, it does represent a majority of the available databases and, to the best of the author's knowledge, all pertinent research for this thesis. Additional studies involving sociological connections with sports and disabilities involving children, leisure activities, and the relationship for motivation and engagement have been excluded (e.g., [63], [64]).

\subsubsection{Increased Force Production versus Decreased Structural Integrity}

Sports with high-impact, high-velocity shoulder-dependent movements, such as sledge hockey, invite risk of increased stresses to the joints, whether they are minimal or maximal, 
acute, or chronic. For shoulder-dependent populations increased force production or elevated reaction force onto the shoulder could be detrimental, foreshadowing the possibility of a stationary lifestyle until healed. Specifically, potential injuries predicted to be caused from skating in sledge hockey are overuse injuries such as muscle and tendon strains, and ligament sprains. These injuries are likely to be caused from the repetitiveness of skating (quick and explosive poling cycles). Bone fractures are not predicted to be caused from skating, but more likely to be a potential injury from collisions (full body contact and/or stick checking).

Unlike in able-bodied sports, sledge hockey has a diverse range of athletes with physical abilities due to congenital and/or injury-induced impairments creating difficulties adapting generalized locomotor skills required for the sport. Kinematic and kinetic analysis of the upper limb's musculoskeletal movement during skating may reveal etiology-specific locomotor patterns that may provide insight for enhancing protection and structural soundness of this joint without compromising its primary function to produce motion.

Subsequent information provides insight to the complexity of the dynamic system (upper limb) enhancing fundamental knowhow and support for the inclusion of baseline parameters within this thesis. Theoretically and mechanically, skating is most efficient as a linear trajectory, as it is the shortest distance between two points. The greatest proportion of the double poling motion occurs in the sagittal plane, supporting a 2-dimensional point of rotation investigation [24], [34], [42], [44], [54], [65]. Additionally, in a non-contractile system, a peak force-generating drop should occur slightly above the horizon with an 
extended arm. However, biomechanical analysis of double poling endorses shortening the lever arm (decrease angle at the elbow) during impact, then increasing the lever arm during force transfer and push-off [24], [25], [42], [44], [51], [54]. Lever arm (moment) length is a key parameter during investigation of impact forces and corresponding moments. Further discussion of moment arm importance within the double poling cycle is outlined in Section 2.2.6.

\subsection{Biomechanical Analysis}

Biomechanics is the study of external and internal forces producing or modifying mechanical movement involving focused investigations on but not limited to the musculoskeletal system within the human body [15], [66]. Biomechanical analysis can be broken up into two distinct subdivisions: 1) kinematics (the study of movement with respect to linear and angular positioning, and their time derivatives), and 2) kinetics (the study of the cause of movement producing linear and angular motion with respect to a NewtonEuler reference system) [15], [67]. Since the primary goal of this research is to obtain kinetic contributions through the use of the acquired kinematic data from the dynamic mechanical system, biomechanical analysis proves to be an appropriate methodology. Biomechanical analysis provides identical methodologies to investigate contractile and non-contractile produced movement; both methods of force production are investigated within this thesis.

\subsubsection{Kinematics Acquired Through Motion Capture}

MOCAP systems use spatial positioning to develop a standard Cartesian co-ordinate 
system in order to perform movement analysis. Orthogonal axes $x, y$ and $z$ are used to quantify linear displacement following the right-hand-rule with $x, y, z>0$. A MOCAP system typically consists of at least three infrared cameras, tracking markers, and corresponding computer software. MOCAP systems combine vectors in the same manner as mechanics to replicate the tracked motion. Appendix A.1 provides detailed explanations regarding the mechanical and anatomical relationship for biomechanical analysis following the International Shoulder Group (ISG) standard protocol [68]. Table 2.2 identifies the axis, plane, and movement relations used for standard biomechanical investigations in conjunction with this thesis' defined global co-ordinate system.

Table 2.2 Global co-ordinate relationships within biomechanical analysis

\begin{tabular}{lllll}
\hline Axis & & Plane & & Movement \\
\hline Anterior-posterior & $x$ & Coronal & $(y, z)$ & Abduction / Adduction \\
Mediolateral & $z$ & Sagittal & $(x, y)$ & Flexion / Extension \\
Longitudinal & $y$ & Transverse & $(x, z)$ & Internal/External Rotation \\
\hline
\end{tabular}

In addition to the single global co-ordinate system, numerous local co-ordinate systems provide focused investigation locations for biomechanical analysis; these locations are tracked with a reflective marker at the joint-centre. Some research suggests the use of a cluster of smaller diameter markers with the joint-centre centrally located [67], [69]-[71]. Motion and skin artifact (signal distortion) are a primary concern with MOCAP systems as the marker is located externally on the skin and used to infer the joint-centre, however, joint-centres do not displace during movement such as the skin does [67], [69]-[71]. Skin movement creates artifact within the acquired marker signal. 
The ISG has derived a standard for motion analysis at each upper limb joint [68]. Insight to the GHJ co-ordinate system used for measurements is provided in Appendix A.1. Each local co-ordinate system presents the opportunity of 6 possibilities for movements within/about the overall co-ordinate system: 3 translational and 3 rotational movements. Degree of freedom $(D o F)$ represents the minimum number of independent variables required to completely describe an object. With regards to the skeleton, joints are axes of rotation from which local co-ordinate systems (LCSs) can be calculated. These LCSs are located within the global co-ordinate system. Following the directionality of the movement under investigation, degrees of freedom at each local origin can then be determined and used during mathematical calculations (i.e., inverse dynamics).

The upper limb resembles similar trajectories to that of an intricate pendulum, where the upper arm, forearm, and hand represent segments within the linked system, their origin of rotation located at the $G H J$-centre ( $D o F 3$ ), elbow ( DoF 2), and wrist ( DoF 3 ), respectively. Displacement about the longitudinal axis (transverse rotation), mediolateral axis (flexion/extension), and anterior-posterior axis (ab/adduction) can be produced at each upper limb joint with the exception of ab/adduction at the elbow.

\subsubsection{Kinetics Acquired Through Motion Capture}

Biomechanical analysis uses kinematics to investigate Newton's Laws of Motion to determine kinetic measurements of the dynamic force system; that is, the Law of Inertia, the Law of Acceleration, and the Law of Action-Reaction. Newtonian Laws can be manipulated amongst the first two Laws of Thermodynamics, that is the Law of 
Conservation of Energy and Carnot's Principle (transduction and entropy), to provide further kinetic evaluations. Appendix A.2 provides detailed explanations regarding the relationships between Newtonian Laws and the Laws of Thermodynamics for the purpose of kinetic measurements.

Biomechanical investigations measure mechanical energy output in terms work $(W)$ and the rate at which work is performed, or power $(P)$. Work (equation (2.1)) and power (equation (2.2)) can be evaluated both linearly and angularly to provide kinetic information about the investigated task. Within the body, angular movement of a force producing system can result in linear displacement of the whole body (e.g., skating in sledge hockey). The cyclical motion of double poling allows the body and sledge to move forward in a straight line. A unilateral stroke produces forces creating the body and sledge to turn/rotate; such nonlinear skating is not discussed within this thesis. The change in mechanical energy is seen as mechanical work.

where,

$$
W=F \Delta s \cos \theta
$$

$W=$ work $\left(\mathrm{kgm}^{2} / \mathrm{s}^{2}=\mathrm{J}\right), F=$ force $\left(\mathrm{kgm} / \mathrm{s}^{2}=\mathrm{N}\right), \Delta s=$ displaced distance $(\mathrm{m})$, and $\theta=$ angle between $F$ and the displacement vector caused by $F$ (degrees or radians).

where,

$$
P=W / \Delta t
$$

$P=\operatorname{power}(\mathrm{W})$, and $\Delta t=$ duration of $W(\mathrm{~s})$.

Mechanical work occurs when an object's total mechanical energy (sum of potential and kinetic energy) changes creating a work-energy relationship through manipulation of the 
Law of Acceleration. The ability to compare work between individuals is provided from this work-energy relationship. From the known duration of work, variations of instantaneous power can be determined, see equation (2.3).

$$
P_{i}=d E / d t
$$

where,

$$
E=\operatorname{energy}(\mathrm{J}) \text {, and } t=\text { time (s) }
$$

The musculoskeletal system's intricate design interlocks body segments using various lever systems designed to enhance the velocity or power of the movement. A simplified lever system within the human body encompasses joints acting as the fulcrum, where muscular force acts on the bone and all distal components (resistance) producing movement with respect to the joint's degree of freedom. Soft skeletal connective tissues link the lever systems together: 1) ligaments attach bones to each other assisting in joint control and congruency, 2) tendons attach muscle to bone providing a direct and efficient transfer of forces, and 3) articulating cartilage provides a minimal-friction surface between bones [15].

All three lever systems are found within the human body, the most prominent being the third-class lever: load-effort-fulcrum from a distal orientation (Table 2.3). A third-class lever within the musculoskeletal system promotes a mechanical advantage for speed, but is inversely disadvantaged for force production (Figure 2.3). From previous literature regarding double poling locomotion produced solely from the shoulder complex, large quantities of force are required to be generated through a joint not architecturally designed to do so [34]. Combining the above-mentioned information regarding the shoulder's structural integrity and range of motion during weight-bearing tasks, a mechanical 
disadvantage at the shoulder exists when concerned with weight-bearing motion requiring elevated power generation. It can be deduced that the primary intention for the shoulder complex is mobility not stability. Mechanically, enough power cannot be generated to create a greater force against the resistance than the force applied to the lever, causing structural weakness within the shoulder complex.

Biomechanical analysis adapted the use of lever systems to evaluate torque about the jointcentre. Torque $(\tau)$ is equivalent to moment $(M)$ in biomechanics and defines the rotational force of an object about a fixed pivot in Newton-metres ( $\mathrm{Nm}$ ), and is defined as the crossproduct of the perpendicular force to the line of action $(\boldsymbol{F})$ and the distance of the force applied to the point of rotation $(r)$, see equation (2.4) and equation (2.5). Specifically, lever arm positioning observed in hip gait analysis has a narrowed angular range compared to shoulder gait. The range boundary effecting moment arm length is anterior to the movement producing joint, at initial surface contact. Typically during walking, leg flexion at initial contact (heel-strike) is closer to the movement producing joint (hip) than compared to the flexed arm (extended elbow) and its movement producing joint (shoulder) at pick-plant during skating (i.e., longer moment arm). From this, it can be interpreted that reaction forces are located closer to the movement producing joint in walking than in skating. This increased angular range has direct effects on torque production about the shoulder during skating in sledge hockey (Figure 2.4). 


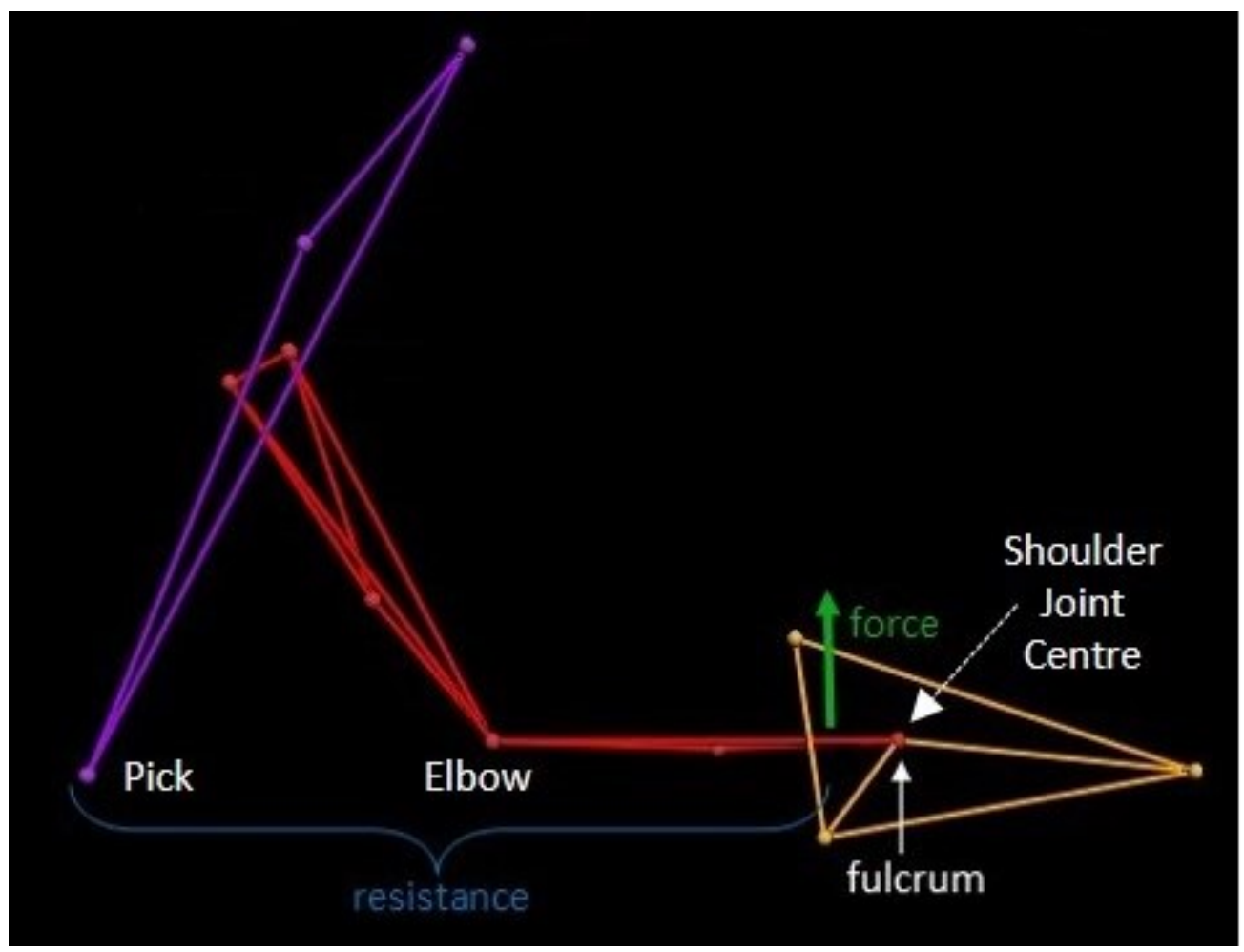

Figure 2.3 MOCAP depiction of the $G H J$ as a third-class lever during the poling cycle in the sport of sledge hockey (force arrow represents direction not magnitude). Pick is point of impact where shoulder joint-centre is point of applied reaction forces. From this, it is clear that an elongated moment arm is created during skating in sledge hockey, foreshadowing potential of overloading injuries.

$$
\begin{gathered}
\boldsymbol{M}=\boldsymbol{r} \times \boldsymbol{F} \\
M=I \alpha
\end{gathered}
$$

where,

$I=$ inertia $\left(\mathrm{kgm}^{2}\right)$, and $\alpha=$ angular acceleration $\left(\mathrm{rad} / \mathrm{s}^{2}\right)$ 


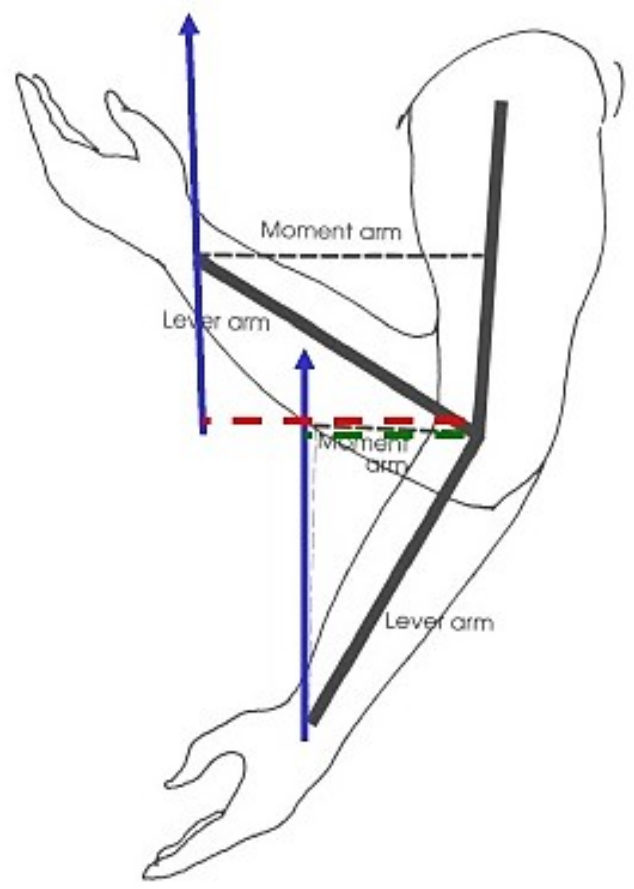

Figure 2.4 Angular range and moment arm length involving elbow flexion and extension, a third-class lever [72]. Since mechanical advantage represents the effectiveness of the lever moving the resistance, a third-class lever, favours the resistance-arm or limb over the force-arm or muscle insertion. Specifically, this figure depicts the importance of the moment arm in relation to the overall system at various instances during a dynamic movement. From this, limb mechanics can be identified as vital components in order to promote optimal force generation and/or transduction. Moment arm (black dash), lever arm (black solid), elbow flexion moment arm (red dash), elbow extension moment arm (green dash), and force arrow (blue arrow).

\subsubsection{Gravitational Energy on a Dynamic System}

Gravitational forces truly represent conservative energy; an object's potential energy decreases as its kinetic energy increases in an identical manner. This is imperative when analyzing tensile forces of a pendulum, or the forces acting on the shoulder in a double poling motion. Each segment of the dynamic mechanical system contributes inertial forces acting about the segment's centre of mass (CoM). A joint separates two segments, and each segment's CoM is determined from standardized anatomical parameters. An object's 
resistance to a change in motion can be defined as its CoM inertia (I). In addition to a CoM, each segment of the upper limb rotates about a radius of gyration $(\rho)$; the distance of the mass from the axis of rotation to a concentrated point. The radius of gyration is not a fixed point like CoM and is dependent upon the axis of rotation. Appendix A.3 provides detailed relations and differences for these key components within biomechanical analysis.

In Study 1 of this thesis, gravitational potential energy from SLAM - 80 can be understood as free fall downward poling within the sport of sledge hockey and used to compare musculoskeletal results from Study 2. This comparison can occur due to the design of $S L A M-80$ in relation to the tested participant population (athletic males roughly the same body type and mass). SLAM - 80 produces limb-restricted results concerning an $80 \mathrm{~kg}$ sledge hockey player in a predicted optimal position for high-velocity skating. Direct focus of the results concerns the point of rotation (shoulder) and initial impact forces. From these results, work to produce the downward motion can be compared. This is seen as the preliminary step for preparation phase investigations, and results will be used in future research as previously mentioned.

\subsubsection{Injury and the Internal Architecture of the Dynamic System}

Structural design of the shoulder ball-and-socket joint encompasses creative supporting architecture while producing the largest range of motion within the human body (Figure 2.5 and Figure 2.6). One of the complex's structural weaknesses is known as shoulder separation and occurs when the clavicle becomes disjoined from the scapula via partial or complete tears of the acromioclavicular and/or coracoclavicular ligaments. 
Acromioclavicular separation was a common sledge hockey specific reported injury, followed by wrist fractures, elbow and wrist tendonitis, lacerations to the upper limbs, and injury to the hands [18], [19]. Mechanisms of injury were determined to be caused by sledge blades, and the positioning of the hand relative to the ice surface [18], [19].

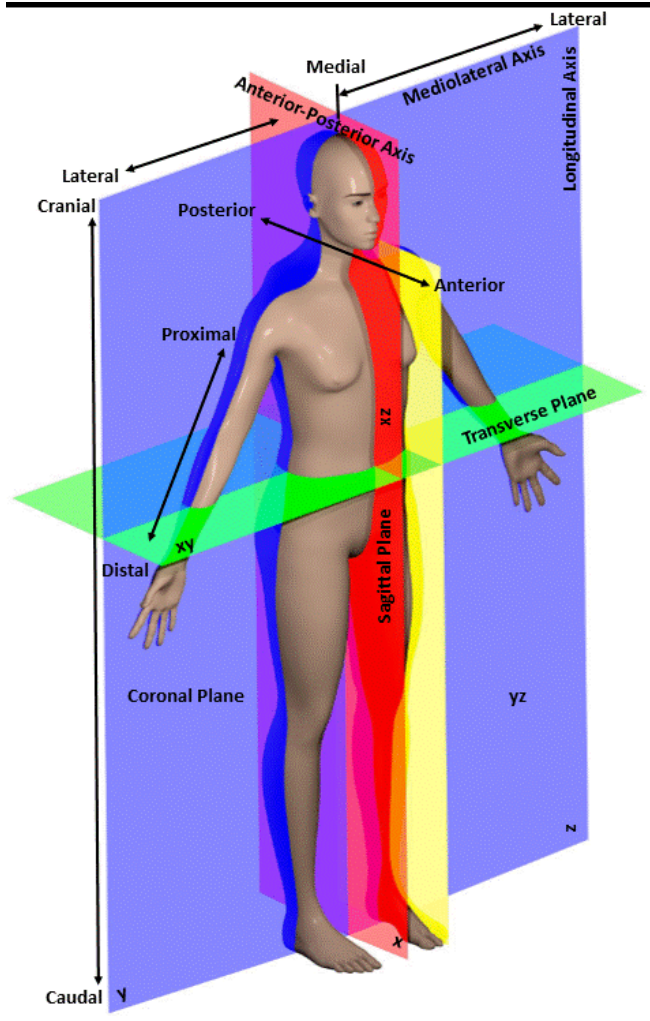

(a)

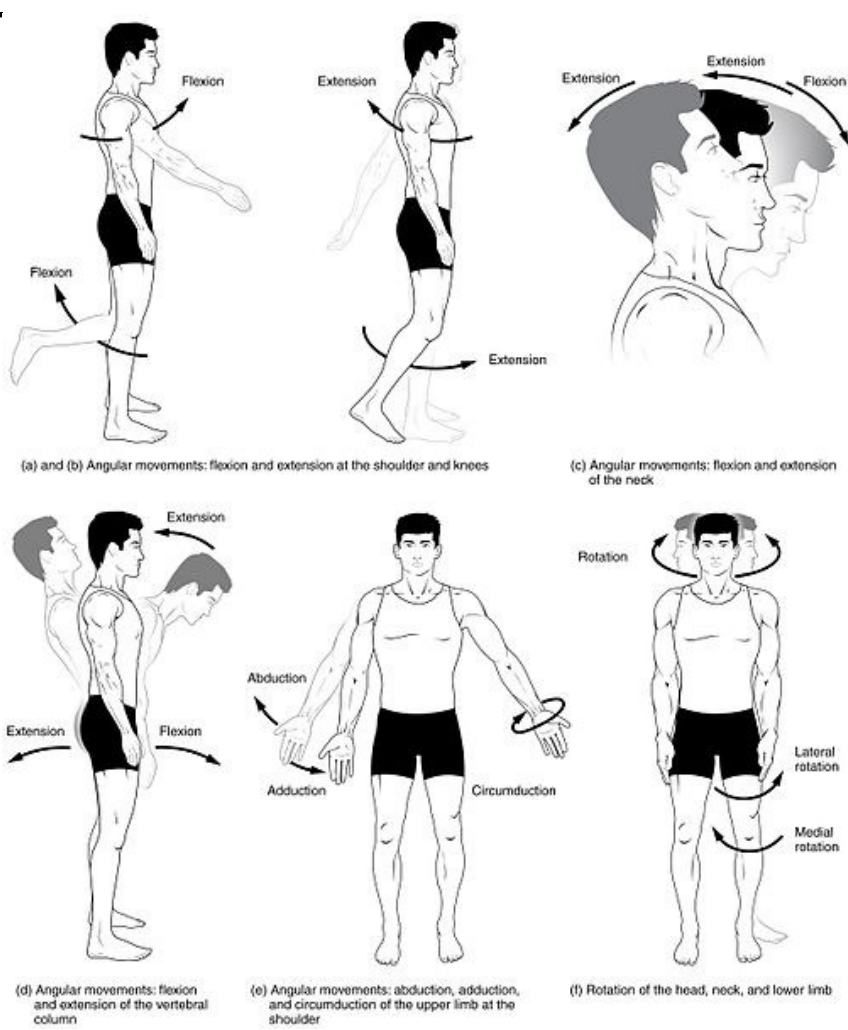

(b)

Figure 2.5 a) Anatomical planes and axes, and (b) Anatomical movements [73], [74]. This thesis focuses biomechanical investigation primarily concerning the sagittal plane ( (a) - red). Motions of importance to skating in sledge in this plane include flexion and extension of the upper limbs and torso ( (b) - first two top and bottom first, respectively). 


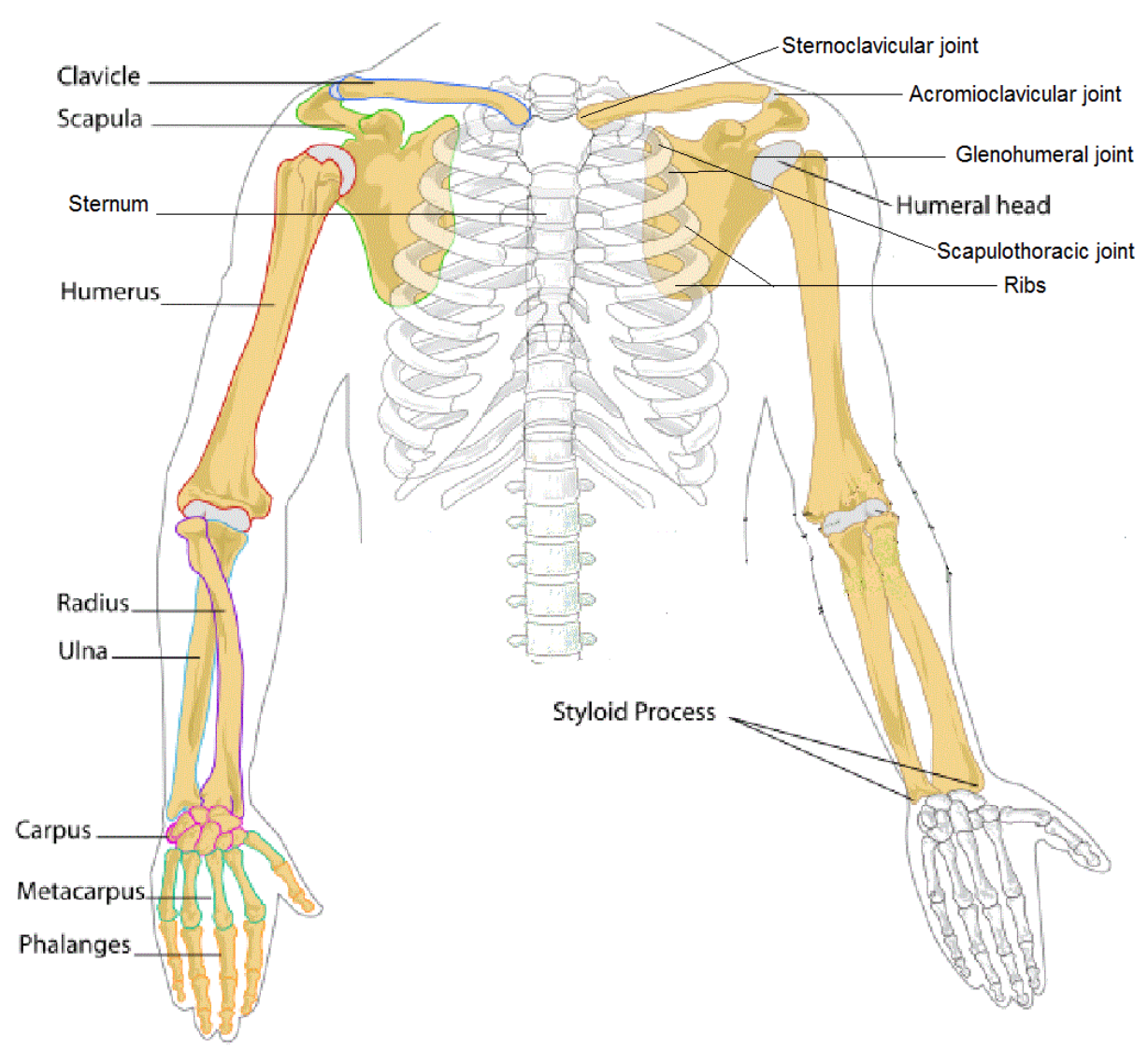

Figure 2.6 Architecture of the shoulder complex and upper limb within the human body [75]. The shoulder girdle is comprised of the clavicle and scapula. The shoulder girdle in conjunction with the humerus creates the $G H J$, and in combination with the thoracic cage, sternoclavicular joint, acromioclavicular joint, and scapulothoracic joint the shoulder complex exists. The anterior superior component of the $G H J$ socket consists of the clavicle, a thin elongated S-shaped bone composed of mostly cortical bone running horizontally to the superior thoracic cage. The scapula, an inverted triangular bone, again mostly composed of cortical bone, is the posterior component of the socket. The humeral head of the long bone known as the humerus (superficial cortical bone accompanied by deep trabecular bone) creates the ball.

The $G H J$ is also the location of the complex's primary structural weakness: shoulder dislocation of the humeral head from the socket. Random-sampling has determined that $1.7 \%$ of the population has succumbed to a dislocation with $90-96 \%$ reported as an anterior dislocation [76], [77]. Reoccurrence of dislocation is extremely high ranging from 
$47-100 \%$ with elevated risks for males between $20-30$ years in fully mobile populations [76]-[81]. Populations relying on the shoulder complex to produce motion have an increased risk of injury due to overloading and/or overuse of this exclusive mobile mechanical system [13], [14], [38], [53], [82]-[88]. As impact in the double poling cycle produces elevated $G R F s$, a heightened risk for anterior dislocations of the $G H J$ is probable due to the joint's inherent structural weakness.

Bone composition dictates the level of deformation the biomaterial can withstand before it succumbs to failure. Bone tissue matrices provide structural support through a combination of rigid inorganic mineral and elastic collagen-based structures capable of remodeling and regeneration. Due to the underlying differences between bone compositions, the resistance to deformation for each bone type greatly differs [15]. Cortical bone has been determined ideal for elevated axial loading (increased force and duration of application) and/or stresses onset by vigorous activity (strength to withstand forces) compared to trabecular bone's spongy composition, which contains the ability for high-energy impact absorption (redirection of impact forces) [15]. Both types of stresses exist within the sport of sledge hockey (a full body contact para-sport); the shoulder is a prominent point for physical contact during the sport, in addition to the shoulder being the major pivot point for poling locomotion.

\subsubsection{Kinetics Acquired Through Surface Electromyography}

Internal forces are generated by skeletal muscles, which have associated electrical activity during contractions. Observation of this electrical activity through biological signal 
acquisition is known as electromyography (EMG). The amplitude of the $E M G$ signal varies with respect to the varying contractile forces exerted from the muscle under investigation, however, it should be noted that $E M G$ is not a representation of muscular force. EMG acquisition can occur deep within the muscular tissue using fine-wire or needle-like electrodes, presenting limitations during dynamic movement, or non-invasively using surface electrodes on the skin. $s E M G$ electrode designs vary from individual electrodes to complete wireless electrode systems. In this thesis, bipolar wireless surface electrodes were used, minimizing motion artifact during off-ice skating. Identifying muscle activation order (co-ordination), frequency, and duration are primary uses for $S E M G$ acquired data [14], [89]-[94]. Section 2.3.3 outlines in greater detail signal acquisition and processing.

During a dynamic movement both an agonist (locomotor) and antagonist (stabilizer) produce force [15], [69]. Co-contraction occurs when the antagonistic pair simultaneously contracts holding a joint in a stable position [15], [69]. Antagonistic pairs produce movements by pulling the shared bone in opposite directions [15], [69]. Co-activation also occurs during dynamic movement. Co-activation is the recruitment of more than one skeletal muscle to produce a movement and/or stabilization [15], [69].

Figure 2.7 and Table 2.3 identify the major locomotors and stabilizers related to the upper limb. Information involving the identified muscles is provided to illustrate the complexity of the musculoskeletal system during skating in sledge hockey. Interpretation of $E M G$ data can improve the understanding of muscle contributions during skating production in sledge 
hockey. Posterior muscle activity is of particular interest as enhanced skating is predicted to occur during a pushing motion (propulsive or more horizontal stick position implying increased force transfer) opposed to the pulling motion (breaking stick position) identified by Hawkeswood et al. [19]. Information from EMG data can also assist with understanding how overuse and/or overloading the upper limbs during skating can potentially cause injury. $S E M G$ were acquired during the data collection phase of this research but these sEMG data are not discussed within this thesis.

Table 2.3 Muscles of interest for this research scope involved with shoulder and elbow movements during skating in sledge hockey

\begin{tabular}{|c|c|c|c|c|c|}
\hline \multicolumn{2}{|l|}{ FORCE } & \multicolumn{2}{|l|}{ LOAD } & \multicolumn{2}{|c|}{ FULCRUM } \\
\hline Muscle & Origin & Insertion & Segment & Joint & Movement \\
\hline \multirow{6}{*}{$\begin{array}{l}\text { Anterior } \\
\text { Deltoid } \\
\text { Pectoralis } \\
\text { Major }\end{array}$} & Shoulder & \multirow[t]{2}{*}{ Humerus } & \multirow[t]{2}{*}{ Humerus } & \multirow[t]{2}{*}{ GHJ } & Flexion \\
\hline & Girdle & & & & Medial Rotation \\
\hline & Clavicle & \multirow[t]{4}{*}{ Humerus } & \multirow[t]{4}{*}{ Humerus } & \multirow[t]{4}{*}{ GHJ } & Flexion \\
\hline & & & & & Adduction \\
\hline & Thoracic & & & & Medial Rotation \\
\hline & Cage & & & & $\begin{array}{l}\text { Horizontal } \\
\text { Flexion }\end{array}$ \\
\hline \multirow{2}{*}{$\begin{array}{l}\text { Short \& Long } \\
\text { Heads Biceps } \\
\text { Brachii }\end{array}$} & \multirow[t]{2}{*}{ Scapula } & \multirow[t]{2}{*}{ Radius } & \multirow[t]{2}{*}{ Radius } & GHJ & Flexion $\left(2^{\text {nd }}\right)$ \\
\hline & & & & Elbow & $\begin{array}{l}\text { Flexion } \\
\text { Supination }\end{array}$ \\
\hline \multirow{4}{*}{$\begin{array}{l}\text { Posterior } \\
\text { Deltoid } \\
\text { Latissimus } \\
\text { Dorsi }\end{array}$} & \multirow{4}{*}{$\begin{array}{l}\text { Shoulder } \\
\text { Girdle } \\
\text { Inferior } \\
\text { Vertebrae }\end{array}$} & \multirow[t]{2}{*}{ Humerus } & \multirow[t]{2}{*}{ Humerus } & \multirow[t]{2}{*}{ GHJ } & Extension \\
\hline & & & & & Lateral Rotation \\
\hline & & \multirow[t]{2}{*}{ Humerus } & \multirow[t]{2}{*}{ Humerus } & \multirow[t]{2}{*}{ GHJ } & Extension \\
\hline & & & & & $\begin{array}{l}\text { Adduction } \\
\text { Medial Rotation }\end{array}$ \\
\hline Long Head & Scapula & \multirow[t]{5}{*}{ Ulna } & \multirow[t]{5}{*}{ Ulna } & GHJ & Extension $\left(2^{\text {nd }}\right)$ \\
\hline Medial \& & Humerus & & & \multirow[t]{4}{*}{ Elbow } & Extension \\
\hline Lateral Heads & & & & & \\
\hline \multirow{2}{*}{\multicolumn{3}{|c|}{$\begin{array}{l}\text { Triceps } \\
\text { Brachii }\end{array}$}} & & & \\
\hline & & & & & \\
\hline Medial & Shoulder & Humerus & Humerus & GHJ & Abduction \\
\hline Deltoid & Girdle & & & & \\
\hline \multirow[t]{2}{*}{ Trapezius } & Superior & \multirow[t]{2}{*}{ Humerus } & \multirow[t]{2}{*}{ Humerus } & \multirow[t]{2}{*}{ GHJ } & Lateral Rotation \\
\hline & Vertebrae & & & & $\begin{array}{l}\text { Horizontal } \\
\text { Extension }\end{array}$ \\
\hline
\end{tabular}



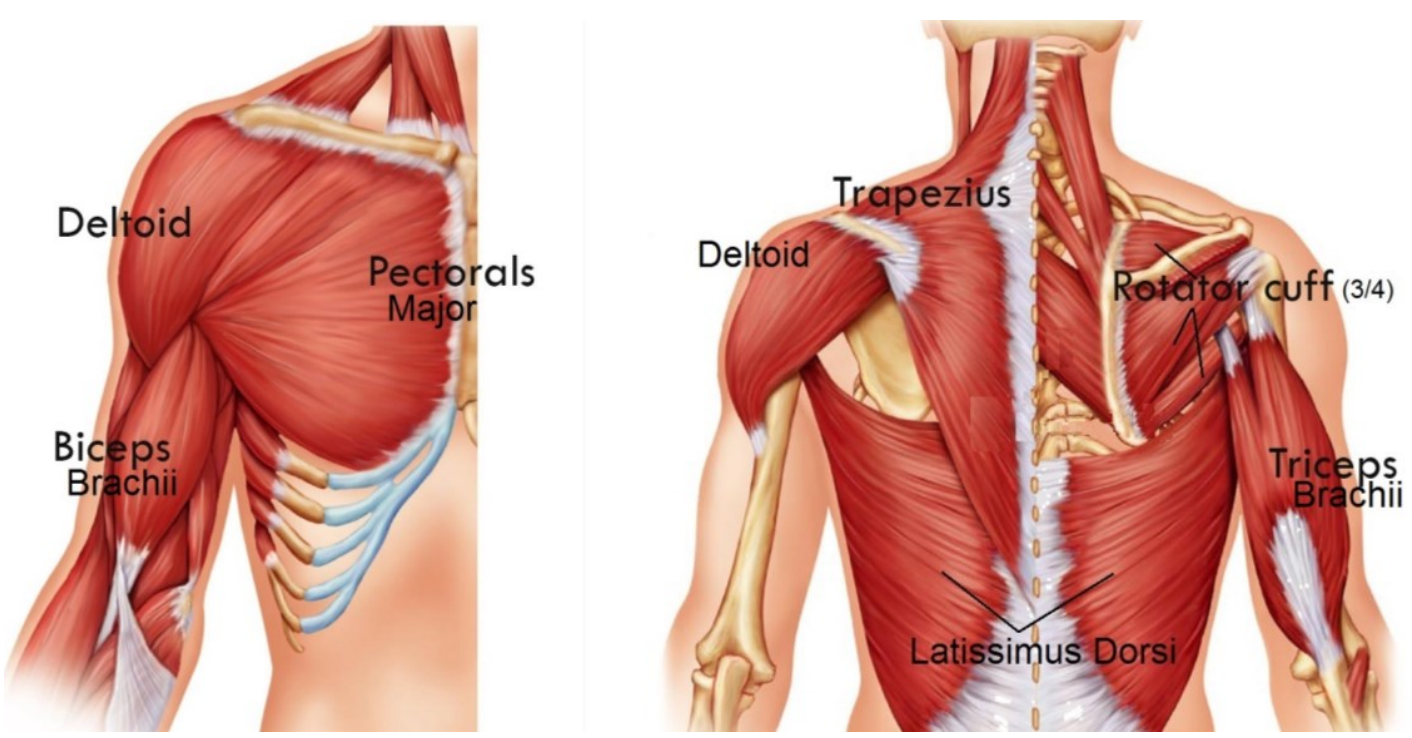

Figure 2.7 Shoulder complex skeletal muscle orientations of particular interest for this research scope involving locomotors and stabilizers producing skating in sledge hockey [95]. Skeletal muscles originate at a bone-tendon interface (origin), produce force to stabilize and/or mobilize their adjoining bone located at the insertion (anchor). A note should be made that the rotator cuff is the primary shoulder stabilizer; however, due to its deep location $s E M G$ acquisition cannot occur.

\subsection{Solving the Dynamic System}

Integration of a MOCAP system, $S E M G$, and force plates provides a diverse and comprehensive data collection set. The following outlines key components to solving a dynamic system via biomechanical computations.

\subsubsection{Inverse Dynamics}

Inverse dynamics utilizes the above information to assist in determining net force and torque at the point of rotation. Inverse dynamics is a mathematical model routinely used to compute internal forces and torque when external forces and inertial properties are known in the absence of closed kinematic chains where the terminal joint is restrained. 


\subsubsection{Ground Reaction Forces Acquired Through Force Plates}

Force plates measure tri-axial GRFS through analog signal acquisition defined by the Law of Action and Reaction. Anterior-posterior (horizontal) force provides propulsive force specifically for the sport of sledge hockey, and is the most useful force when analyzing this motion. Longitudinal (vertical) force provides the downward force of the stroke cycle, where mediolateral force provides left and right forces from the midline. Specifically for this thesis, the existence of mediolateral forces can be attributed to maintaining balance and bilateral asymmetries in force production. Total (resultant) reaction force is the summation of all reaction forces per point of contact (i.e., left stick, right stick). Resultant force is also a key indicator of performance output, where all force analyses provide information regarding their specific anatomical axes, allowing heightened interpretations of the skating motion with regards to injury prevention. Centre of pressure $(\mathrm{CoP})$ is a value calculated from the GRFs and moments recorded from the force plates, which is also useful in understanding a subject's balance and congruency of task.

Complete biomechanical analysis of a double poling cycle requires multiple force plates. Para-sports present difficulty in GRFs acquisition due to multiple contact points (left and right stick, sledge chassis, and front of sledge if it is required for balance) compared to bipedal walking.

\subsubsection{Signal Acquisition and Processing}

Integrating $M O C A P, S E M G$, and force plate data requires a conversion from analog (continuous-time) to digital (discrete-time) permitting comparable analysis of the linked 
mechanical system. Each signal was acquired in this thesis using a different sampling rate, requiring mathematical manipulations to appropriately analyze these data on the same time scale. Appendix A.4 provides detailed information regarding signal acquisition and processing.

The sampling rate $\left(f_{s}\right)$ is the recording rate (frequency) of the signal. In biomechanics, the sampling rate is generally $5-10$ times greater than the Nyquist frequency ensuring a true representation of the signal (encompassing outlying peak values) [67], [69], [90]. Voluntary human locomotion produces movement at frequencies less than $10 \mathrm{~Hz}$; however, sampling rates for trajectory acquisition within biomechanics typically range between $200 \mathrm{~Hz}$ (walking) and $400 \mathrm{~Hz}$ (running) [67], [69]. From Section 2.1.4, Table 2.1, poling impact forces generated GRFS as high as $475 \mathrm{~N}$ [34], [54]. Based upon standard sampling rate practices incorporating the Nyquist frequency, force plates sampling frequencies should be set no lower than $1000 \mathrm{~Hz}$ when investigating poling impact GRFS, ensuring that outlying spiking forces can be acquired (e.g., [65]). Holmberg et al. set strain gauge sampling frequencies to $2000 \mathrm{~Hz}$ ensuring adequate measurement of poling impact forces [42]. Motor-unit contraction frequencies peak around $250 \mathrm{~Hz}$ suggesting a sampling frequency for $s E M G$ to range between $500 \mathrm{~Hz}$ (static) and $1000 \mathrm{~Hz}$ (dynamic) [67], [69], [90].

\subsubsection{Signal Filtering}

Filtering removes distortion from the raw signal by attenuating the undesired frequencies [89], [90], [92], [93], [96]. There are four types of filter: 1) high-pass (unchanged high 
frequencies), 2) low-pass (unchanged low frequencies), 3) band-pass (unchanged frequencies within the range), and 4) band-stop or notch (attenuated frequencies within the range). Filters can often be implemented digitally, so that they can be applied post acquisition if required. Not all acquired signals benefit from filtering. For example, high frequency signals, such as the impact forces seen in the double poling cycle can be negatively affected by filtering (e.g., undesired attenuation high frequency components).

Zero-lag filters can be used to preserve the signal phase, which can be important to maintain the relative timing between data (e.g., $S E M G$ and MOCAP data). Zero-lag filters run the filter over the signal then repeats the filter in the reverse direction removing the phaseshift. Filters have orders determining the sharpness of the slope cut-off when smoothing out the sinusoidal wave. $2^{\text {nd }}$ and $4^{\text {th }}$ order filters are suggested for biomechanical analysis, with the understanding that applying a zero-lag filter doubles the originally set $\operatorname{order}\left(2^{\text {nd }}\right.$ becomes $\left.4^{\text {th }}\right)$ [67], [69], [90], [92], [93], [96].

For $s E M G$, filtering out baseline wandering $(<1 \mathrm{~Hz})$, power line interference $(60 \mathrm{~Hz})$, and motion artifact (typically $<20 \mathrm{~Hz}$ ) is common. Appendix A.4 outlines specific mathematical applications regarding signal filtering, specifically Fast Fourier Transform (FFT), Shannon's Reconstruction Formula, and signal-to-noise ratio (SNR; the ratio of signal power to noise power, often expressed in decibels).

\subsubsection{Processing and Publication of Acquired Signals}

MOCAP systems are equipped with corresponding computer software that can reproduce 
and animate the body segments estimated from the 3-dimensional locations of the surface markers during the acquired movement. Most MOCAP software allows for the integration of $s E M G$ and force plate systems to synchronize the data. In some instances, $s E M G$ data are simultaneously acquired via system-specific software then integrated post acquisition to the MOCAP and/or force plate data.

Processing of the acquired signal(s) involves some and/or all of the following: 1) MOCAP marker labeling, 2) trajectory smoothing (manual smoothing of a specific section of the surface marker trajectory using toolbox features), 3) signal filtering (applied to the entire digital signal independently for each system, e.g., all trajectory data), 4) data cropping, 5) event marking, and 6) model processing (produces pre-set kinetics based on the acquired data). Researchers have designed numerous software-based mathematical models integrating joint and segment kinetics based on marker placement and biomechanical knowledge of segment movement. MOCAP software integrates validated models in order to produce the desired kinetics of the acquired motion. Measurements are based on participant specific anthropometrics manually entered into the model. Due to the infancy of sledge hockey in the elite sporting world, a viable computer generated sledge hockey model does not currently exist.

Raw or processed signals (MOCAP, $S E M G$, and force plate) can be processed using MATLAB. MATLAB scripts can be created/implemented to analyze the signals on an individual or integrated basis, allowing for isolated or complete reviewable discrete results. MATLAB based toolbox software has been developed enhancing biomechanical analysis 
and publication of results through the use of such software as OpenSim. Typically, such software requires model-processed data (.mod files) to integrate the musculoskeletal graphics enabling researchers to enhance the presentation and publication of their findings. Again due to its infancy, integration of sledge hockey results with such software is not currently possible.

\subsection{Summary}

Downward poling in the sport of sledge hockey is produced solely from the shoulder. Anatomically, the shoulder produces 3 degrees of freedom where mechanically, straightline forward displacement produces optimal forward force transfer. Increase in angular range also increases the potential for injury to the producing joint strictly based on moment arm length. Lever arm (i.e., elbow angle) changes affecting the derived moment arm, in turn effecting torque produced about the shoulder and its production of optimal forward locomotion. The upper limbs are linked mechanical systems, which are not required to move bilaterally, and are the major contributors when producing weight-bearing movement in the sport of sledge hockey. Variations within each joint angle alter the transmission of force in-vivo in turn altering the final outcome, forward displacement. The sticks are an extension of the linked mechanical system and must be investigated independently and as a combination in order to deduce optimal forward skating in the sport of sledge hockey.

The integration of $M O C A P, s E M G$, and force plates can provide useful data used to compute inverse dynamics of the shoulder and the respected kinetics during skating in sport of sledge hockey. Increased knowledge regarding the biomechanics required to produce 
skating is provided by data presented in this thesis. This knowledge may help reduce injury and optimize skating within the sport of sledge hockey. Results may also be transferrable to shoulder-dependent populations providing knowledge to improve quality of life through prevention, rehabilitation, and positively manipulated task performance. 


\section{Chapter: Study 1: Solid-Static System}

This chapter consists of six sections. Section 3.1 outlines the rationale for supporting the use of a mechanical prototype (SLAM - 80) to identify and determine key biomechanical parameters required to initiate propulsion during skating in sledge hockey.

Section 3.2 provides the methodology used to develop the structural design of SLAM - 80 from relevant scientific anthropometric investigations.

Section 3.3 provides the methodology used to determine baseline measures from SLAM 80. Section 3.3.1 outlines the MOCAP - force plate system used during data acquisition. Section 3.3.2 outlines the testing protocol defining elbow angles and drop heights. Section 3.3.3 outlines data acquisition and processing specifics used for analysis. Section 3.3.4 outlines the applied mathematical model developed to solve the estimated kinetics of each $S L A M-80$ trial.

Section 3.4 provides results from $S L A M-80$. Section 3.4 .1 provides sagittal plane trajectory interpretations specifically concerning pick impact angle in relation to lever arm (i.e., elbow angle). Section 3.4.2 provides impact force analysis specifically concerning peak impact force. Section 3.4.3 provides the calculated $J R F_{\text {Shoulder }}$ and its interpretations specifically concerning calculated $J R F_{\text {Shoulder }}$ at peak impact force. Section 3.4.4 provides information regarding two key measures for performance and injury prevention analysis. This section provides the estimated torque about the shoulder, and impulse calculated from pick force plate contact and the respective reaction forces. Section 3.4.5 provides the 
estimated limb segment power at the shoulder and its interpretations specifically concerning estimated peak power and specific power.

Sections 3.5 discusses the use of $S L A M-80$ as an appropriate mechanical model to produce initial impact forces during skating in sledge hockey. Interpretation of Study 1 results are derived from previous identified analogous researches (Chapter 2). Increased impact force at initial contact is identified as a potential mechanism for injury as a result of skating in sledge hockey.

Section 3.6 summarizes the results and provides conclusions for baseline interpretations involving skating initiation in sledge hockey. This section also discusses how the newly defined baselines are involved within the remaining components of this thesis (Chapter 5), and with future research investigations (Chapter 6).

\subsection{A Relationship between Free Fall Downward Poling in Sledge Hockey and Propulsive Force Transfer}

Improving the current understanding on how to produce optimal performance is necessary in order for sledge hockey players to excel within their sport. To the author's best knowledge, currently, there are no published biomechanical investigations involving skating in sledge hockey. The purpose of this study was to evaluate kinematic and kinetic characteristics of the motion that initiates skating in sledge hockey. 
Specifically, the $S L A M-80$ model is used to define the initiation range of the downward stroke, and to measure pick impact angle ranges that contribute to force production and transfer from predetermined elbow angle ranges.

\subsubsection{The Solid-Static System}

A solid-static system representing the inertial properties of the average male's upper arm was achieved by developing an anatomically correct prototype (SLAM-80). The prototype is made of solid segments, with elbow and wrist-stick joints fixed (static). Fixed joints create a static system from point of rotation to the pick of stick. SLAM -80 in its test position resembles a simplified male athlete during late preparation phase for skating in sledge hockey (Figure 3.1).

By developing and validating SLAM - 80 (this study, Study 1), further research involving initial impact in skating can occur without introducing additional stresses onto a shoulderdependent individual. Results from Study 1 are used as a baseline comparison against musculoskeletal produced poling (Chapter 4, Study 2) to further understand skating initiation in the sport of sledge hockey, and as a mechanism of potential injury (see Chapter $5)$. 


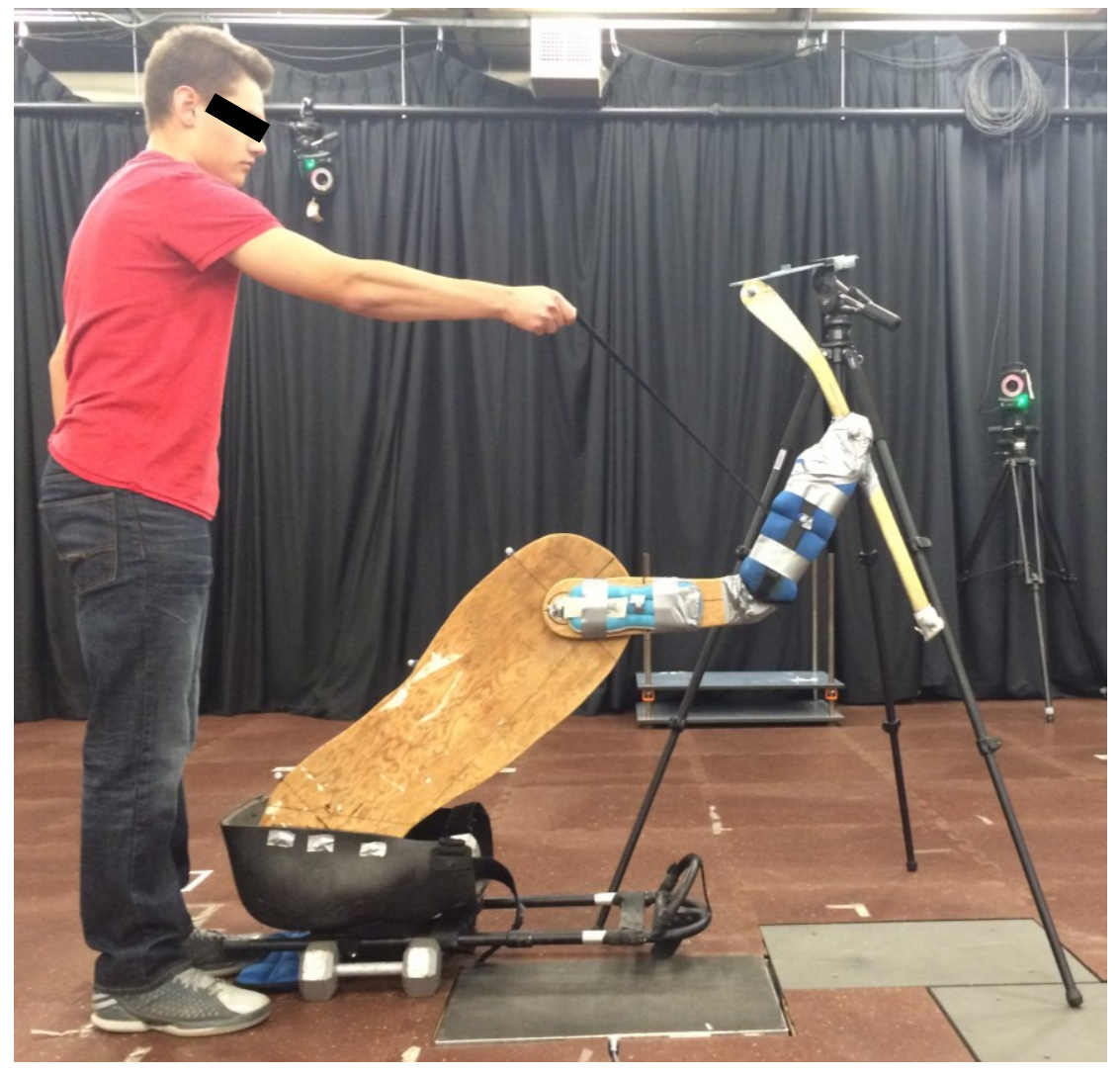

Figure 3.1 SLAM - 80 horizontal start position (shoulder joint-centre to elbow joint-centre) located centrally in a 10-camera MOCAP system. Impact forces were obtained from a single force plate protected by a rubber wavy mat (black force plate closest to sledge). SLAM - 80 freestanding in the late preparation phase was tested.

\subsubsection{Baseline Deviations and Skating Initiation in Sledge Hockey}

Baseline measures are standard measurements in which other similar-tasked evaluations can be compared against. Baseline measures can be defined as initial measurements, the minimum measurement, or the average measurement of the desired task. In this thesis, impact baseline measurements are defined as: 1) sagittal plane pick impact angles, 2) 3dimensional impact forces (Note: SLAM - 80 produced motion is in the sagittal plane, although reaction forces are measured in 3-dimensions, a majority of the produced motion would ideally be 2-dimensions), and 3) sagittal plane produced kinetics involving the 
shoulder, of an average $80 \mathrm{~kg}$ male model $(S L A M-80)$ during a single downward free fall poling stroke in the sport of sledge hockey. Independent variables include elbow angle and drop height. SLAM - 80 is more controllable (e.g., establishing fixed angles) and repeatable (trial to trial) simplifying trajectory patterns. Simplified trajectory patterns improve kinetic computations of the downward pole elevating the understanding of the force producing system (arm and stick) as a whole.

\subsubsection{Understanding Lever Arm to Enhance Force Production Initiation in Sledge} Hockey

From Chapter 2, lever arm has a direct effect on torque produced at the point of rotation. An extended forearm increases the upper limb segment length, which theoretically increases the torque/moment applied at the shoulder joint at impact. Additionally, lever arm affects pick impact angle, where theoretically a more acute (horizontal) angle with respect to the ground produces a decreased moment arm. Theoretically, an elevated initial drop height produces increased impact force. Mechanically, the benefits of elevated stroke initiation are unknown for the sport of sledge hockey.

\subsubsection{Simulation versus Applied Mechanical Models}

Biomechanical computer software are developed from available experimental evidence [97], [98]. Musculoskeletal models are designed to compute kinematic and kinetic measures for simulated dynamic movements [97], [98]. There is currently no standardized framework for such biomechanical software [97]. Vaughan outlines key limitations for the use of computer simulation for biomechanical analysis from aggregated researches, 
identifying validated computer models as the major weakness [98]. In agreement, Panjabi argues that mathematical models (only a set of equations) are linked to reality through the intent to model physical properties, and success is proven by suitable validation [99]. It is further argued that no perfect validation is possible [99]. Algorithms developed/composed for computer simulations are done so from known instances/researches, however, the purpose of the simulation is to predict behaviour in unknown instances [99].

Vaughan discusses limitations for the use of computer simulation in sport biomechanics, particularly, the incorporation of practical settings into the simulation of real-life events [98]. Practical settings in some sports change with each competition/venue. Specifically, ice level temperature in hockey rinks affect the co-efficient of friction between the blades and picks against the ice surface, in turn affecting the work duty cycle. Vaughan also outlines advantages for the use of validated computer simulations [98]. Table 3.1 outlines the advantages and disadvantages of computer simulation identified by Vaughn [98, p. 377].

Results from computer simulations are typically presented in academic literature, which is outside the scope of most coaches/training personnel [98]. Vaughan suggests that the complexity of the findings be presented in easily understood conclusions appropriate for both academics and non-academics [98]. Specifically, Vaughan identified the use of a wooden model and elastic bands used by Bauer in order to present the sophistication of the mathematic model of a gymnasts moving about a horizontal bar [98], [100]. From this, a simplified mechanical model can be understood as the foundation to understanding 
biomechanical movement. This mechanical model could then be used as the initial step for developing a valid computer model.

Table 3.1 Advantages and disadvantages of computer simulation [98, p. 377].

Advantages Disadvantages

1 Safety: no hazardous experiments for athlete

Validation is difficult, and incorrect models prejudice results

2 Time: many different simulations Advanced mathematics are often performed quickly required for the user

3 Optimal performance can be predicted Results are often difficult to translate to practicality

4 Expense: no need to build different physical models

5 Microcomputers: cheap, easy to use good graphic display

The following seven inferences are derived from the literature presented in this thesis supporting the use of $S L A M-80$ in lieu of computer software:

1) There is currently no computer model for sledge hockey.

2) Computer simulations use pre-set anthropometric datasets typically from fully mobile populations [67], [69], [97], [101]-[103].

3) Scientific data are scarce for sledge hockey, a sport encompassing a wide variety of anthropometric parameters and physical limitations.

4) Simplified mechanical systems can provide heightened understanding of complex dynamic movements, which can also be seen as the preliminary step to validating a computer model.

5) There is currently minimal literature investigating the biomechanics of 
seated double poling [11], [24], [44].

6) The preparation phase is dependent upon pole length for seated double poling [11], [24], [44].

7) Knowledge of biomechanical trends identifying the preparation phase are limited for all forms of double poling [11], [24], [34], [44].

\subsection{Methodology: SLAM - 80 Design}

Acknowledgement is made to Professor Bjarki Hallgrimsson (School of Industrial Design, Carleton University) for his guidance during the initial stages of the design for SLAM 80.

Sledge hockey is an upper body physically demanding para-sport, implying elite players are likely to have upper body mesomorph physiques. Due to the solid-like composition, mesomorph body segments deform less during motion improving kinematic and kinetic interpretations [15], [67], [69]. Standardized body segments have been identified for various percentiles of body compositions [22]. Mesomorph body composition resembles the first percentile for standardized body segments [22]. From this, the structural makeup for $S L A M-80$ was established.

\subsubsection{U.S. Military Anthropometric Data}

Military combat injuries are one example of the current increase in the shoulder-dependent population [2]. Military personnel are also likely to have upper body mesomorph physiques [20], [21]. The U.S. Military published a document providing complete anthropometrics 
for the mesomorph body type [20], [21]. Between 2009 and 2010 the U.S. Army and Marine Corp surveyed 1301 male personnel with 786 participants ranging between the ages of $21-30$ [20], [21]. The structural design for $S L A M-80$ was constructed using this supporting literature as the most current and relevant peer-reviewed anthropometric analysis. From U.S. Military data, average body mass $(B W)$, seated height, seated acromial height, and shoulder-elbow and forearm lengths were acquired [20], [21]. Table 3.2 outlines the anthropometrics used to design $S L A M-80$ from joint-centre to joint-centre, and the respective standard segment $\operatorname{CoM}$ locations for the average male between $21-30$ years of age [20]-[22], [67].

Table 3.2 SLAM - 80 anthropomorphic measurements [20], [22], [67]

Measurement CoM Length from Proximal

(m) (\% of segment)

\begin{tabular}{lll}
\hline Seated Height & 0.92 & 0.626 \\
Hip to GHJ & 0.62 & \\
GHJ to Elbow & 0.36 & 0.436 \\
Elbow to Centre of Grip & 0.35 & $0.430^{*}$
\end{tabular}

* forearm plus hand

\subsubsection{Segment Architecture \& Morphology}

Segment architecture was established by integrating the values from Table 3.2 with AutoCAD blueprints for the mesomorph adult male (Table 3.3) [20]-[22], [67]. Replicating the AutoCAD blueprints, segment length $>$ joint-centre to joint-centre as indicated in Figure 3.2 (a) [22]. Mathematical manipulations were required in order to maintain proper scaling within the segment's structural design. Factor increases per 
centimeter (AutoCAD measurements) required to recreate the segments to scale were $3.75 \mathrm{~cm}, 3.60 \mathrm{~cm}$, and $3.90 \mathrm{~cm}$ for the torso, upper arm, and forearm, respectively. Since the upper limb was the only component of interest, torso length was created to $90 \mathrm{~cm}$ for simplicity of model design. A cardboard blueprint was designed to replicate the AutoCAD segments' exact architectures. Upon confirmation of the measurements a working foam prototype was designed to determine congruency of the desired task, followed by a working wood prototype $<0.02 \mathrm{~m}$ thick. An AutoCAD blueprint for $S L A M-80$ is presented in Appendix B.1.

Flexible-weights were used to approximate limb morphology in order to preserve the radius of gyration and inertial properties within the dynamic system. Two $1.30 \mathrm{~kg}$ weights were attached to the upper arm in a stretched out fashion with an overlap at CoM (medial and lateral orientation), and a $1.20 \mathrm{~kg}$ weight was attached to the forearm in a stretched out fashion (lateral orientation) (Figure 3.2). A note should be made that moment of inertia for $S L A M-80$ was not calculated. From this, even though the mass of the arm segment for SLAM - 80 and an average male arm are the same (mass distributed similarly), the moments of inertia are not matched.

Static distal joint design allowed for slight absorption properties resembling similar biomaterial properties produced from the diarthodial joints. This was caused by uncontrollable deformation of axle hole by the screw (axel). The female side of a Velcro strap was attached at the forearm's CoM and used to raise the dynamic system to various drop heights (Figure 3.1). Two plastic washers $(4.00 \mathrm{~cm}$ in diameter) were used to decrease 
friction at the dynamic joint.

Table 3.3 Segment architecture for SLAM - 80 [20], [22], [67]

\begin{tabular}{lllll}
\hline Segment & $\begin{array}{l}\text { Length } \\
(\mathbf{m})\end{array}$ & $\begin{array}{l}\text { Joint-Centre } \\
\text { to Joint- } \\
\text { Centre } \\
\text { Length (m) }\end{array}$ & $\begin{array}{l}\text { CoM Length } \\
\text { from } \\
\text { Proximal (m) }\end{array}$ & $\begin{array}{l}\text { Segment } \\
\text { Weight (kg) }\end{array}$ \\
\hline Upper Arm & 0.44 & 0.36 & 0.19 & 2.24 \\
Forearm & 0.44 & 0.35 & 0.19 & 1.28 \\
Stick & $(0.45+$ & $0.40^{* *}$ & $0.32^{* * *}$ & $1.00+$ \\
& $0.21 \cos \theta)^{*}$ & & $0.48^{* * * *}$ \\
\hline
\end{tabular}

* shaft length plus (blade times interior angle between shaft and blade)

${ }^{* *}$ wrist to pick length $\quad{ }^{* * *}$ CoM length from distal end ${ }^{* * * * *}$ stick plus hand

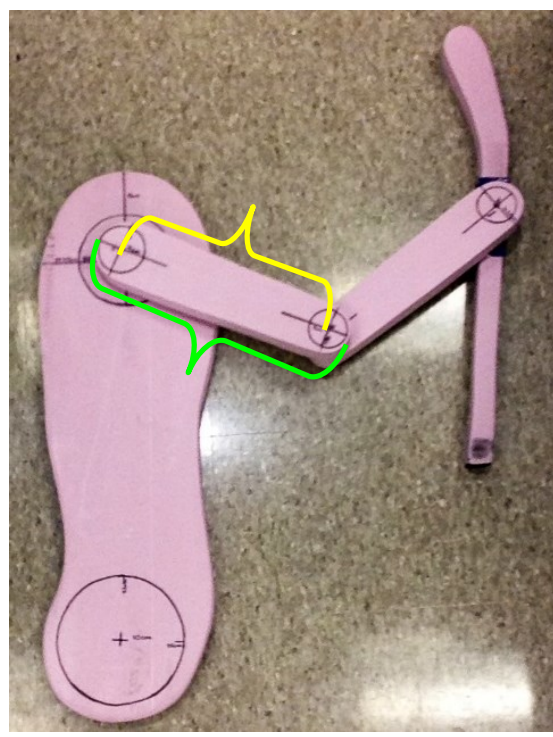

(a)

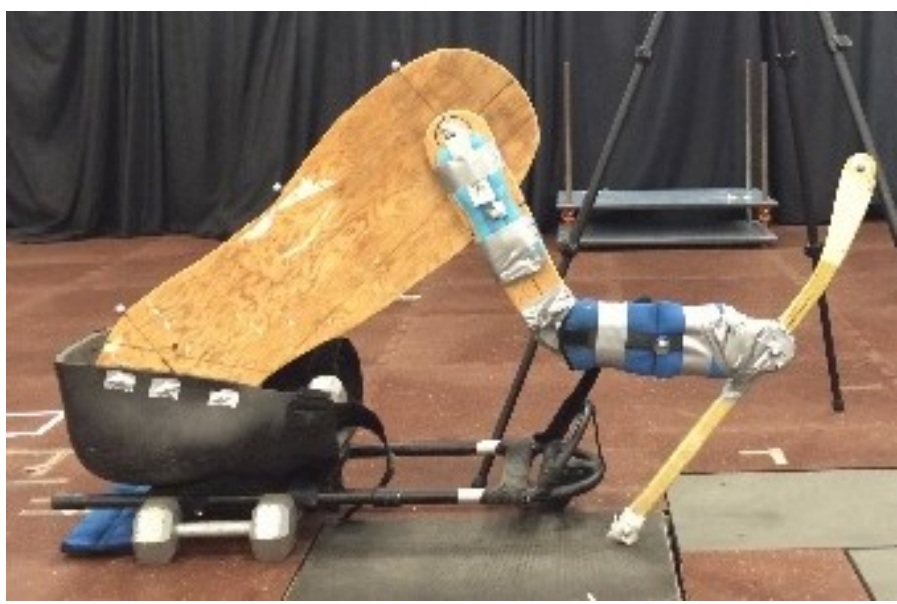

(b)

Figure 3.2 (a) $S L A M$ - 80 foam prototype created to ensure task congruency and structural design. Key measurements included segment length (green) and joint-centre to joint-centre (yellow), and (b) Sagittal plane impact position for a typical $S L A M-80$ trial. Pick contact was the only contact force acquired from the force plate.

\subsubsection{Torso Angle and Elbow Angle Range}

Torso angle and elbow angle range were established from alpine sit-ski literature 
investigating sit-ski design controlling $C o M$ and inertia, in conjunction with pilot on-ice sledge hockey investigations conducted by the author [23], [25]. Alpine sit-skiers require increased balance in the bucket, improving control as they produce highly explosive and agile poling motions, similar to sledge hockey [25]. A sit-ski bucket has a very different design (chair-like with specific leg supports) compared to a sledge hockey sledge (seat with open leg supports); however, both sports utilize the same increased torso flexion during initiation of explosive poling bursts [23], [25]. Therefore, SLAM - 80 was fastened to a sledge hockey sledge at a fixed hip angle $+40^{\circ}$ from the horizon, with weights placed in the bucket to stabilize the sledge.

Elbow angle range was developed from the same sit-ski literature. Using an elbow jointcentre screw, the elbow angle was set at $120^{\circ}, 135^{\circ}$, or $150^{\circ}$ (fully extended elbow equals $180^{\circ}$ ), creating flexed, middle, and extended experimental conditions, respectively [25]. Wrist-stick angle was secured with a wrist joint-centre screw, and a forearm-stick screw creating a $45^{\circ}$ angle inferiorly between the wrist and stick. Wrist-stick angle was used to create the midpoint in which pick-plant angle could be produced to create forward propulsion.

\subsubsection{Previous Disseminations of $S L A M-80$ Results}

SLAM - 80 was first introduced at the Own the Podium Sports Innovation Summit (SPIN 2014), held in Montreal, Canada, in an unpublished abstract, "Propulsion in Sledge Hockey: A Biomechanical Analysis to Define Gait", on and off-ice data outlining the importance of baseline measures during this specific dynamic movement was presented as 
a poster by the author [104].

The importance of baseline measures during dynamic movement was discussed at the Women in Science and Engineering National Conference (WISE-NC 2015), held in Toronto, Canada, in an unpublished abstract, "Shoulder joint produced weight-bearing mobility: Defining Gait", the development of SLAM - 80 was introduced and presented as a poster by the author [105].

Validation for the working prototype was published in the International Federation for Medical and Biological Engineering (IFMBE) Proceedings at the World Congress on Medical Physics and Biomedical Engineering (IUPESM) held in Toronto, Canada, in the paper, "Validating a Solid-Static Single-Armed Male Prototype Tasked to Produce Dynamic Movement from the Shoulder through the Preparation Phase" [32]. Findings to improve investigation included:

1) Additional plate-weights (opposed to dumbbells) should be placed within the bucket to promote increased stability during free fall. This limitation was brought about by the un-weighted torso and narrow 2-dimensional design of SLAM -80 .

2) Drop heights should be increased from $\pm 10^{\circ}$ to $\pm 30^{\circ}$ with respect to the horizon. This adjustment was supported by sit-ski literature [25].

3) A tripod indicating drop height initiation should be used to improve consistency between drops.

4) Force plate sampling frequency recommended to be set at $2000 \mathrm{~Hz}$ due to the short duration of impact. 
5) Filters suggested to be removed from force plate raw signals, again due to the short duration of impact.

Validation for the initial test determining baseline measures was published in the ISBS Conference Proceedings at the $33^{\text {rd }}$ International Conference on Biomechanics in Sport (ISBS) held in Poitiers, France, in the paper, "Investigating the Seated Double Poling Cycle: Identifying Baseline Measures for the Preparation Phase" [33]. New findings to improve investigation included:

1) Increase MOCAP trajectory sampling frequency to $250 \mathrm{~Hz}$.

2) Drop heights classifications recommended to be: above horizon, at horizon, and below horizon instead of segment angles.

Test-retest data were presented at Own the Podium SPIN 2015 held in Toronto, Canada, as an unpublished abstract, "Baseline Impact reaction Forces in a Seated Downward Short Pole Free-Drop: A Biomechanical Investigation of Elbow Angle upon Impact in the Sport of Sledge Hockey" [106]. Initial resultant GRF findings were presented as a poster by the author. All previously outlined suggestions are reflected within the dataset presented in this thesis.

\subsection{Methodology: Acquisition Apparatus and SLAM - 80 Protocol}

Baseline measures are derived from impact force through the use of a MOCAP - force plate system, and $S L A M-80$. Dependent variables are manipulated within the testing session to provide multiple measurable baseline parameters. The following outlines the 
methodologies implemented to acquire and derive results from $S L A M-80$ data.

\subsubsection{MOCAP, Force Plates, and $S L A M-80$ Set-up}

All data were collected at the Human Movement Biomechanics Laboratory, Lees Campus

- University of Ottawa, Ottawa, Canada. A Vicon MOCAP system consisting of 10 infrared MX13 cameras outlined a $2 \mathrm{~m} \times 2 \mathrm{~m} \times 3 \mathrm{~m}$ capture zone [103]. Vicon Nexus MOCAP software (version 1.8.3, Vicon, West Way Oxford, UK) was the corresponding computer software [103]. A Bertec FP4060-08 force plate was centrally located within the capture area [107]. A thin wavy rubber pad $<1 \mathrm{~mm}$ lined the surface of the force plate to provide protection from the pick upon impact. Additionally, the rubber padding improves the pick contact interface by decreasing slippage and providing an interface more closely related to that of the stick-ice interface (pick digs into the ice surface). In contrast, the addition of the rubber padding also dampens the impact signal acquired; the extent of this dampening is unknown. A sledge hockey pick consists of four triangular points cornering the width of the pick (similar transverse dimension to the stick). The pick of the stick was taped with cotton hockey tape to minimize the sharpness of the pick-points against the force plate and maintain equipment congruency. Rubber cane foots were suggested as a secondary option but disregarded due to their significantly greater contact area. A tripod with a stretched out ruler provided a consistent stick blade start height between trials. Wrist-stick angle was set to $45^{\circ}$.

Trajectory markers (14 $\mathrm{mm}$ diameter reflective spheres) were placed down the spine of SLAM - 80 (indicating the posterior superior iliac spine (PSIS), thoracic vertebrae 
10 (T10), and cervical vertebrae 7 (C7), and the anterior midline indicating the sternum and clavicle) in order to recreate the torso via computer software. Markers were then placed laterally at the shoulder, elbow and wrist joint-centre (screws), and at upper arm and forearm CoM (pre-indicated on SLAM - 80). Finally, a blade tip marker and pick marker were place laterally on the stick. Pick marker was placed at the pick-stick interface (screw). Data were required to be negatively shifted the distance from the pick marker to the tip of the pick in order to deduce true impact locations $(y=0.000 \mathrm{~m})$. This distance was measured and marked in ink to ensure exact placement/replacement. Marker placements are displayed in Figure 3.2 (b) (grey spheres).

\subsubsection{Elbow Angles, Overall Design, and Protocol}

With three fixed elbow angles $\left(E ; 120^{\circ}, 135^{\circ}\right.$, and $\left.150^{\circ}\right)$ and three drop heights $\left(\sim 30^{\circ}\right.$ above $(A b v$, Figure $3.3(\mathrm{a}))$, at $\sim 0^{\circ}(\operatorname{Horz}$, Figure $3.3(\mathrm{~b}))$, and $\sim-30^{\circ}$ below (Blw, Figure 3.3 (c)) the horizon, there was a total of nine test configurations. Drop height angle was measured using a hand-sized protractor parallel to the horizon at the $G H J$ screw through to the angle measured in line with the midline drawn on the upper arm segment. The meter stick-tripod system was raised/lowered to the tip of the stick blade during the first measurement of each drop height, allowing for a more consistent starting angle among the drop height trials. Each configuration was tested until three usable trials were acquired. Failed trials consisted of $S L A M-80$ falling over before impact, or interference from the tripod leg during the downward drop. For a given elbow angle, three usable trials were collected for a given drop height before adjusting the height, starting with $A b v$ and ending with Blw. All trials for all drop heights were collected for a given elbow angle before 
adjusting the elbow angle, starting with $E 120^{\circ}$ and ending with $E 150^{\circ}$.

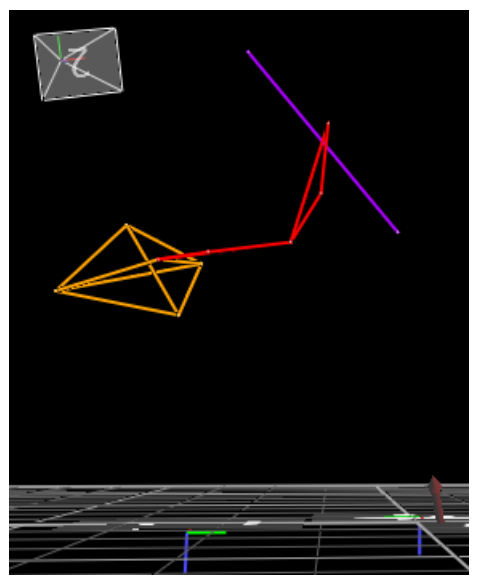

(a)

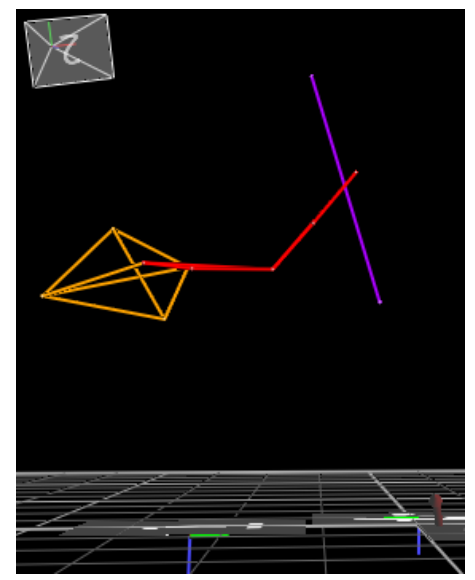

(b)

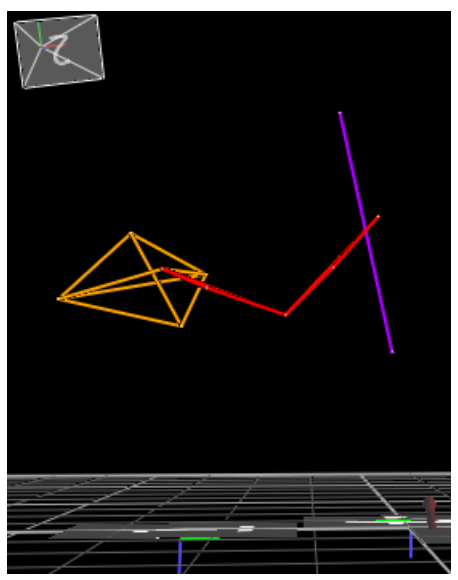

(c)

Figure 3.3 MOCAP computer regenerated SLAM - 80 start heights and elbow angles: (a) Mid elbow above drop height, (b) Extended elbow horizon drop height, and (c) Flexed elbow below drop height start position.

\subsubsection{Data Acquisition, Processing, and Publication}

The force plate sampling rate was set to $2000 \mathrm{~Hz}$ and the raw unfiltered signals were used in the analysis. Marker trajectory data were collected at a sampling rate of $250 \mathrm{~Hz}$. A lowpass zero-lag $2^{\text {nd }}$ order Butterworth digital filter, with a cut-off frequency of $12 \mathrm{~Hz}$ was applied in MATLAB (version R2014b, Mathworks, Natick MA, USA) to the trajectory data [108]. Reaction force trial length was determined from initial contact $\left(t_{\text {Impact }} \Rightarrow F_{x} \neq\right.$ $\left.0 \mathrm{~N}\left\|F_{y} \neq 0 \mathrm{~N}\right\| F_{z} \neq 0 \mathrm{~N}\right)$ to point of recoil $\left(t_{\text {Recoil }} \Rightarrow F_{x}=F_{y}=F_{z}=0 \mathrm{~N}\right)$. Start of trial was determined by the preceding $y$ trajectory local maximum to initial contact $\left(t_{0}=\right.$ $y_{\text {Max }}$, where $t_{0}<t_{\text {Impact }}$ ), and understood as the start of the downward free fall. Brief static-hold, pre-drop buffer zones were implemented to ensure complete acquisition of trial data. End of trial was equivalent to point of recoil for this study $\left(t_{f}=t_{\text {Recoil }}\right)$. MATLAB 
code was written to produce sagittal plane trajectories, peak force, torque, impulse, rate of force development, and power at the shoulder for the limb-segment.

\subsubsection{SLAM - 80 Applied Mathematical Model}

Applied mathematical models are used in various disciplines such as engineering for the purpose of computing practical problems motivated from mathematical based theories [109]. An inverse dynamic mathematical model was developed, scripted into MATLAB, and used to estimate respective kinetics involving the shoulder. Estimated kinetics were calculated from measured peak resultant impact reaction forces produced from the respective $S L A M-80$ tests (mean of three trials for each condition). Kinematic values used in the model were the marker locations acquired from the MOCAP system.

Appendix C.1 was used to compute sagittal plane net force and torque at the GHJ-centre from the reaction forces at the point of impact to initiation of recoil with averaged data collected from $S L A M-80$. Appendix C.2 was used to compute total power at the shoulder for $S L A M-80$ as a continuation from the derived data.

\subsection{Results}

Results are presented in a step-wise analytical approach discussing first the kinematics then the respective derived kinetics of $S L A M-80$ acquired data. The inverse dynamic mathematical model was used to estimate kinetic values of each trial at its respective peak impact force. Peak impact force is defined by the respective test's peak resultant impact force (impact force is defined as mean resultant $G R F$ ). Results are used to determine the 
reliability and validity of $S L A M-80$ 's ability to produce baseline measures for the sport of sledge hockey.

\subsubsection{Sagittal Plane Pick Impact Angles for Free Fall Downward Poling in Sledge}

\section{Hockey}

Anterior-posterior and longitudinal trajectory data were processed analyzing the free fall downward pole for each drop height, and elbow angle (Figure 3.4). From trajectory data, pick angles at peak impact force (pick impact angles) were computed. Mean and standard deviation $(S D)$ of the three trials for each condition were calculated (Table 3.4). A flexed elbow $\left(E 120^{\circ}\right)$ produces the least forward force transferring (propulsive) pick impact angles $\left(52^{\circ} \pm 1^{\circ}\right)$ compared to all other elbow positions. The extended elbow $\left(E 150^{\circ}\right)$ produces the most propulsive pick impact angles $\left(37^{\circ} \pm 3^{\circ}\right)$. Within the extended elbow drop heights a noticeable difference occurs, with less propulsive pick impact angles at the above $\left(40^{\circ} \pm 2^{\circ}\right)$ drop height compared to the remaining two $\left(35^{\circ} \pm 1^{\circ}\right)$. Mid elbow $\left(E 135^{\circ}\right)$ pick impact angles are similar to above extended elbow pick impact angles.

Upon review of MOCAP data, all sagittal plane elbow angles are similar among their respective trials. $S L A M-80$ designed biomaterial absorption properties only allowed peak impact elbow angles to range $\pm 0.4^{\circ}, \pm 1.6^{\circ}$, and $\pm 1.0^{\circ}$, for their respective elbow angle (flexed, mid, and extended elbow respectively). Sagittal plane wrist-stick peak impact angles are $48^{\circ} \pm 0^{\circ}$ for the flexed elbow, $48^{\circ} \pm 0^{\circ}$ mid elbow, and $49^{\circ} \pm 1^{\circ}$ extended elbow. Maximal right lateral pick displacement throughout each extended elbow trial are similar for the above $(2.6 \mathrm{~cm})$, horizon $(2.6 \mathrm{~cm})$, and below $(3.1 \mathrm{~cm})$ drop heights. 


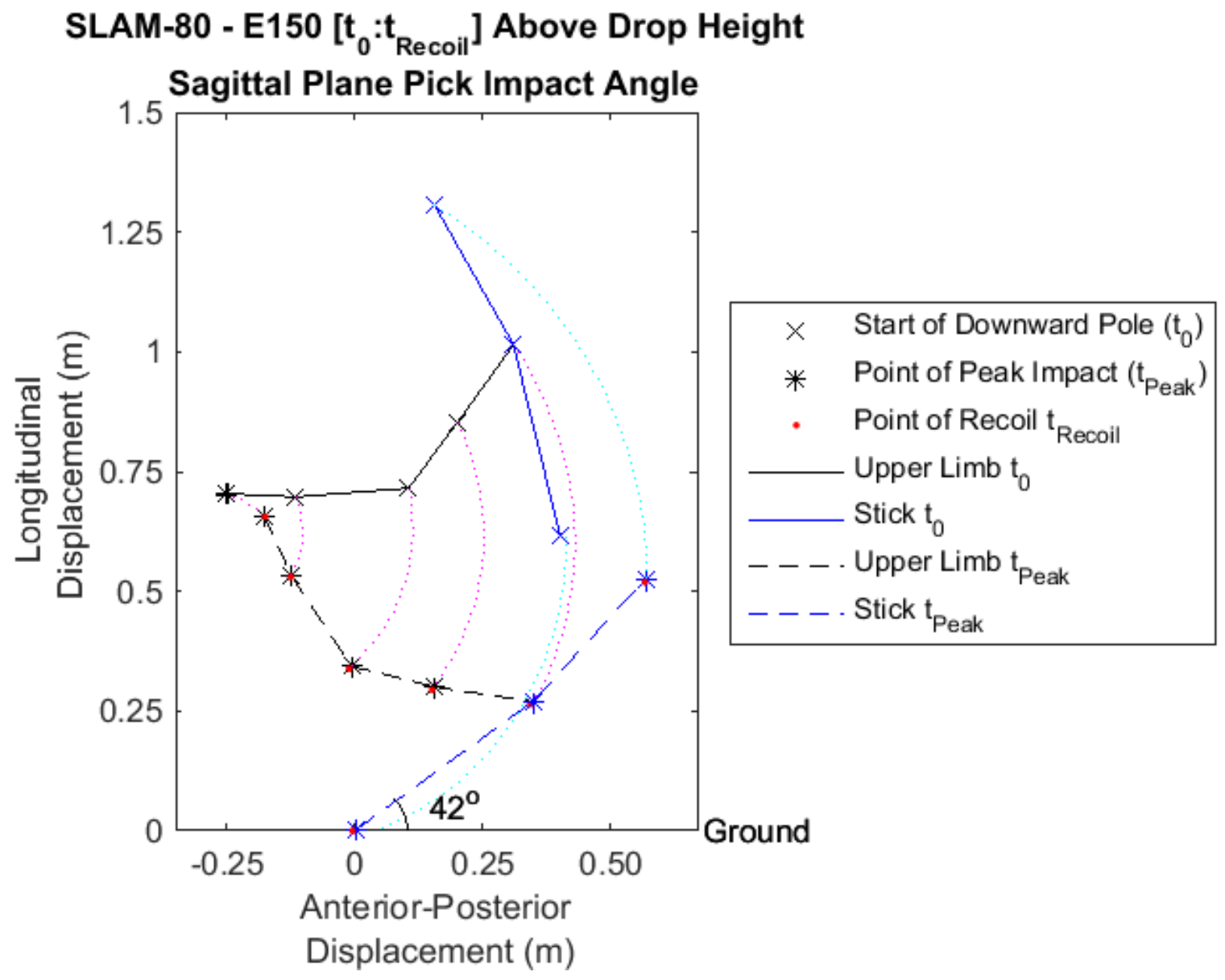

Figure 3.4 $S L A M-80 E 150^{\circ}$ trial three above drop height sagittal plane trajectories illustrating pick impact angle for downward poling; black - limb, blue - stick, solid $-t_{0}$, and dashed $t_{\text {peak }}$. Mid and extended elbows produced the most acute pick impact angles implying the most propulsive stick positions for stroke initiation in sledge hockey at a $40^{\circ}$ torso angle.

Table 3.4 Pick impact angles for all SLAM - 80 trials

\begin{tabular}{llll}
\hline $\begin{array}{l}\mathbf{n}=\mathbf{3} \text { per } \\
\text { condition }\end{array}$ & $\boldsymbol{E 1 2 0}^{\circ}$ & $\boldsymbol{E 1 3 5}^{\circ}$ & $\boldsymbol{E 1 5 0}^{\circ}$ \\
\hline \hline Above $\left({ }^{\circ}\right)$ & $52 \pm 1.0$ & $40 \pm 0.4$ & $40 \pm 2.4$ \\
Horizon $\left({ }^{\circ}\right)$ & $53 \pm 0.9$ & $40 \pm 3.5$ & $35 \pm 1.0$ \\
Below $\left({ }^{\circ}\right)$ & $52 \pm 0.1$ & $39 \pm 1.2$ & $35 \pm 0.9$ \\
\hline \hline Mean $\pm \boldsymbol{S D}\left({ }^{\circ}\right)$ & $\mathbf{5 2} \pm \mathbf{0 . 8}$ & $\mathbf{4 0} \pm \mathbf{1 . 9}$ & $\mathbf{3 7} \pm \mathbf{2 . 9}$ \\
\hline
\end{tabular}


From the above information, an unique opportunity has presented itself. As the extended elbow created the most propulsive pick impact angles, and theoretically a more horizontal angle is more propulsive, extending the elbow can be interpreted as an ideal motion for skating initiation in the sport of sledge hockey. From the differences between extended elbow pick impact angles at the various drop heights, Study 1 results can be further investigated to potentially provide heightened information regarding pick impact angles and enhanced skating in sledge hockey. From these interpretations, analysis with focus on the above-mentioned relations may provide heighten information regarding motion production, lever arm (i.e., elbow angle), and/or potential stick impact introduced injuries for the sport of sledge hockey. Discussion involving the above-mentioned inferences occurs in Section 3.5.

\subsubsection{Impact Force for Free Fall Downward Poling in Sledge Hockey}

Data from SLAM - 80 impact forces establish the foundation for kinetic analysis of the mechanical system during the downward pole and force transfer initiation in the sport of sledge hockey. From Table 3.5, lever arm (i.e., elbow angle) did make a slight difference when initiating horizontal force in sledge hockey. Specifically, a flexed elbow produces less horizontal force than the mid $(-212 \mathrm{~N})$ and extended $(-113 \mathrm{~N})$ elbow. Investigating extended elbow drop height results, the above drop height $(1158 \mathrm{~N} \pm 17 \mathrm{~N})$ produces the greatest horizontal impact force compared to the remaining two $(992 \mathrm{~N} \pm 16 \mathrm{~N}$ and $1027 \mathrm{~N} \pm 80 \mathrm{~N}$, horizon and below respectively). No overall difference was observed when initiating vertical force between lever arms (i.e., elbow angle). Further investigation involving horizontal force throughout the contact phase occurs in Chapter 5. Discussion 
involving rate of force development is provided in Section 3.5.

Table 3.5 Component impact force analysis for $S L A M-80$

\begin{tabular}{llll}
\hline $\mathbf{n}=\mathbf{3}$ per condition & \multicolumn{1}{c}{$\boldsymbol{E 1 2 0 ^ { \circ }}$} & \multicolumn{1}{c}{$\boldsymbol{E 1 3 5}^{\circ}$} & \multicolumn{1}{c}{$\boldsymbol{E 1 5 0}^{\circ}$} \\
\hline \hline Peak Horizontal Force (N) & 1027 & 1271 & 1170 \\
Above Mean Horizontal Force (N) & $995 \pm 32$ & $1253 \pm 17$ & $1158 \pm 17$ \\
Horizon Mean Horizontal Force (N) & $952 \pm 31$ & $1024 \pm 86$ & $992 \pm 16$ \\
Below Mean Horizontal Force (N) & $892 \pm 19$ & $1196 \pm 48$ & $1027 \pm 80$ \\
\hline \hline Mean Horizontal Force (N) & $\mathbf{9 4 6} \pm \mathbf{5 1}$ & $\mathbf{1 1 5 8} \pm \mathbf{1 1 5}$ & $\mathbf{1 0 5 9} \pm \mathbf{8 7}$ \\
\hline Peak Vertical Force (N) & 1314 & 1483 & 1469 \\
Above Mean Vertical Force (N) & $1257 \pm 55$ & $1362 \pm 109$ & $1430 \pm 35$ \\
Horizon Mean Vertical Force (N) & $1201 \pm 66$ & $1076 \pm 77$ & $1187 \pm 23$ \\
Below Mean Vertical Force (N) & $1158 \pm 38$ & $1162 \pm 80$ & $1164 \pm 65$ \\
\hline \hline Mean Vertical Force (N) & $\mathbf{1 2 0 5} \pm \mathbf{6 4}$ & $\mathbf{1 2 0 0} \pm \mathbf{1 4 9}$ & $\mathbf{1 2 6 0} \pm \mathbf{1 3 3}$ \\
\hline
\end{tabular}

From Table 3.5, sagittal plane mean peak resultant impact force vector directions can be computed via simple trigonometry (identical angle measurement method to impact angles). From Table 3.6, mean peak resultant impact force (mean impact) per elbow angle was determined to be $1514 \mathrm{~N} \pm 80 \mathrm{~N}(1.9 \times B W$ and $28 \times \operatorname{Limb})$ and direction $52^{\circ} \pm 1^{\circ}$ for the flexed elbow, $1669 \mathrm{~N} \pm 178 \mathrm{~N}(2.1 \times B W$ and $31 \times \mathrm{Limb})$ and direction $43^{\circ} \pm 3^{\circ}$ for the mid elbow, and $1625 \mathrm{~N} \pm 152 \mathrm{~N}(2.1 \times B W$ and $30 \times$ Limb $)$ and direction $47^{\circ} \pm 3^{\circ}$ for the extended elbow. Regardless of lever arm (i.e., elbow angle), the above drop height produces approximately $230 \mathrm{~N}$ greater peak resultant impact force than compared to the other two drop heights. A flexed elbow initiates skating with less peak resultant impact force than the mid $(-297 \mathrm{~N})$ and extended elbow $(-252 \mathrm{~N})$, however, overall there is no difference between elbow angles during force transfer initiation in sledge hockey. The greatest peak resultant impact force was determined to be $1924 \mathrm{~N}(2.5 \times$ $B W$ and $36 \times \operatorname{Limb})$ and direction $49^{\circ}$ from $E 135^{\circ}$ trial one. From Table 3.7, peak 
resultant impact force for all elbow angles was $1810 \mathrm{~N}(2.3 \times \mathrm{BW}$ and $34 \times \mathrm{Limb})$ and direction $49^{\circ}$.

Table 3.6 Resultant impact force analysis for $S L A M-80$

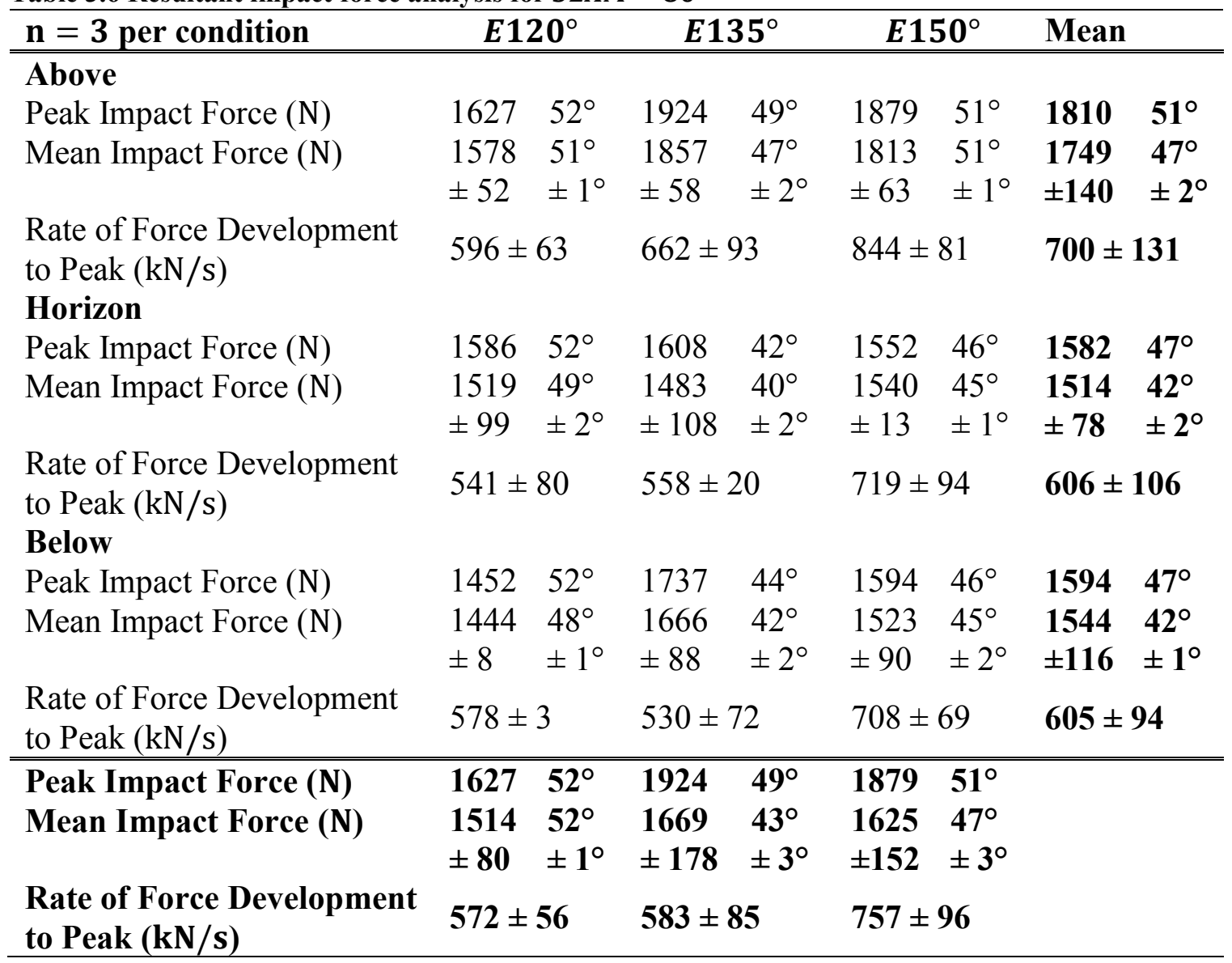

Table 3.7 Mean of all resultant impact forces produced by $S L A M-80$

\begin{tabular}{lll}
\hline \hline $\mathbf{n}=\mathbf{2 7}$ & & \\
\hline \hline Peak Impact Force (N) & 1810 & $49^{\circ}$ \\
Mean Impact Force (N) & $1602 \pm 137$ & $44^{\circ} \pm 3^{\circ}$ \\
Rate of Force Development to Peak (kN/s) & $637 \pm 116$ & \\
\hline
\end{tabular}


For a flexed elbow, decrease in drop height was proportional to a decrease in peak resultant impact force, which was expected. For a mid and extended elbow, a drop height approximately at shoulder level proved to produce the least amount of peak resultant impact force for their respective elbow angles. Figure 3.5 illustrates a typical resultant force curve observed within Study 1 (black solid).

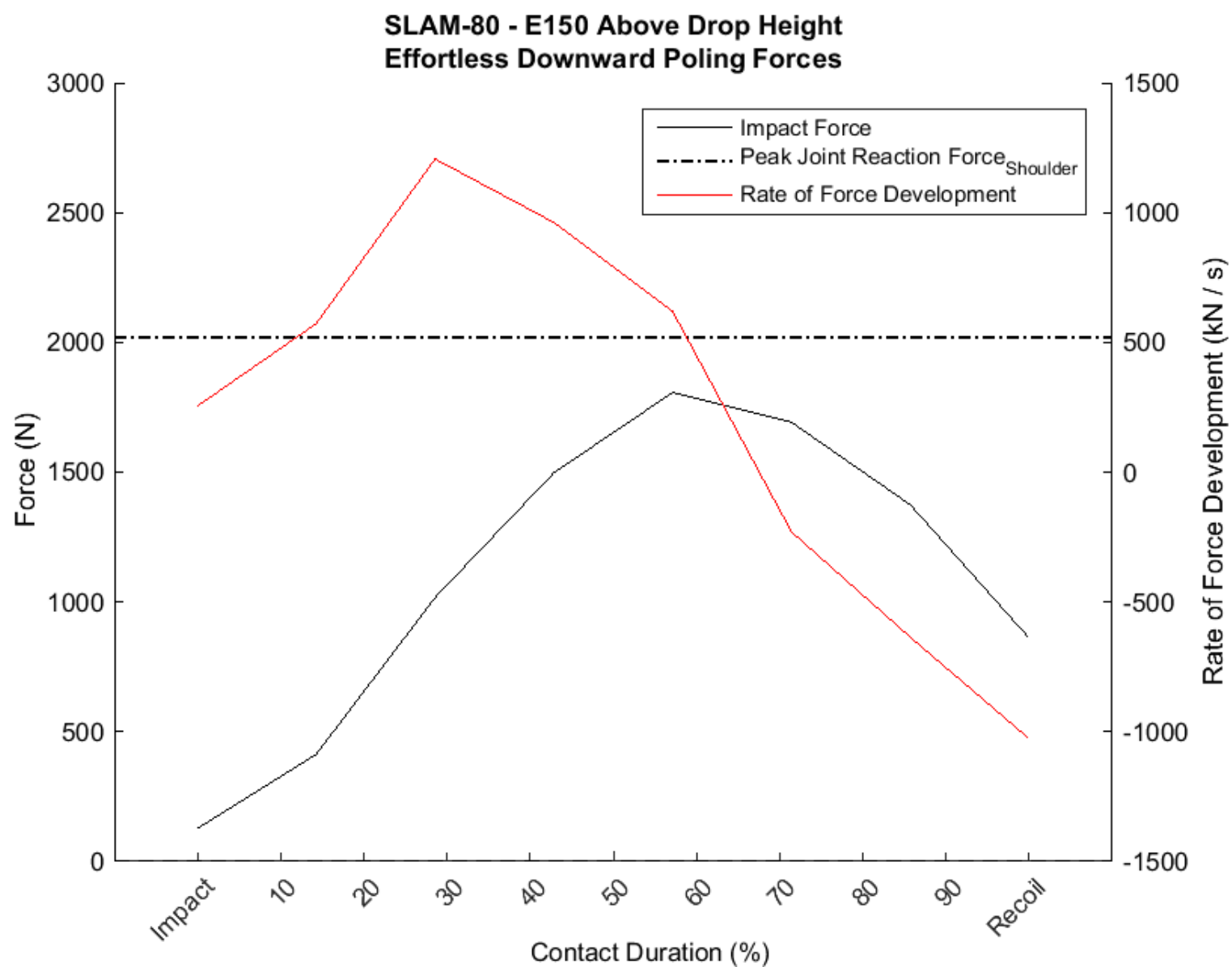

Figure 3.5 $S L A M-80 E 150^{\circ}$ above drop height (trial three) resultant impact force curve (black solid), and peak $J R F_{\text {Shoulder }}$ (black dashed $=2021 \mathrm{~N}$ ) (left axis), and rate of force development (red solid) (right axis). Aggregation of these data represents force involved with the shoulder during free fall forward force transfer initiation in sledge hockey. 


\subsubsection{Estimated Joint Reaction Force at the Shoulder for Free Fall Downward Poling in Sledge Hockey}

From Newton's third law, joint reaction force $\left(J R F_{\text {Shoulder }}\right.$, force at the shoulder derived from inverse dynamics for $S L A M-80$ ) is a result of impact force created at the point of rotation for the mechanical limb system. The following discusses the estimated $J R F_{\text {Shoulder }}$ of the limb segment at the defined peak impact force for $S L A M-80$. Results are presented as the difference of $J R F_{\text {Shoulder }}$ and peak impact force against peak impact force (Figure 3.6). From Figure 3.6, there is an obvious increase of force at the point of rotation caused by impact $\left(J R F_{\text {Shoulder }}>\right.$ peak impact force). Comparing Table 3.8 to Table 3.6, and from Figure 3.6, a flexed elbow (x) produces the greatest increases of force onto the shoulder as a result of impact. From Figure 3.6, mean increase in force for a flexed elbow (black $\mathrm{x}$ ) is $280 \mathrm{~N}$, mid elbow (black *) $277 \mathrm{~N}$, and extended elbow (black o) $259 \mathrm{~N}$. Overall on average (black + ), a $272 \mathrm{~N}$ increase in force was observed at the shoulder as a result of impact for all elbow angles. Investigating extended elbow drop height results, a greater increase in force is transmitted back onto the shoulder at the above $(2087 \mathrm{~N} \pm$ $74 \mathrm{~N}$ and direction $\left.46^{\circ}\right)$ drop height compared to the remaining two $(1800 \mathrm{~N} \pm 21 \mathrm{~N}$ and direction $44^{\circ}$ for the horizon, and $1767 \mathrm{~N} \pm 91 \mathrm{~N}$ and direction $43^{\circ}$ for below). Moreover, overall, the extended elbow (o) produces impact forces with lower increases in $J R F_{\text {Shoulder }}$ implying extending the arm could be a safer position at impact than a flexed elbow. 
Table 3.8 Estimated $J R F_{\text {Shoulder }}$ from $S L A M-80$ at peak impact force

\begin{tabular}{|c|c|c|c|c|c|c|}
\hline$n=3$ per condition & \multicolumn{2}{|c|}{$E 120^{\circ}$} & \multicolumn{2}{|c|}{$E 135^{\circ}$} & \multicolumn{2}{|c|}{$E 150^{\circ}$} \\
\hline Above $(\mathrm{N})$ & $\begin{array}{l}1881 \pm \\
49\end{array}$ & $\begin{array}{l}37^{\circ} \\
\pm 1^{\circ}\end{array}$ & $\begin{array}{l}2152 \pm \\
58\end{array}$ & $\begin{array}{l}47^{\circ} \\
\pm 1^{\circ}\end{array}$ & $\begin{array}{l}2087 \pm \\
74\end{array}$ & $\begin{array}{l}46^{\circ} \\
\pm 1^{\circ}\end{array}$ \\
\hline Horizon $(\mathrm{N})$ & $\begin{array}{l}1794 \pm \\
120\end{array}$ & $\begin{array}{l}36^{\circ} \\
\pm 2^{\circ}\end{array}$ & $\begin{array}{l}1751 \pm \\
106\end{array}$ & $\begin{array}{l}41^{\circ} \\
\pm 1^{\circ}\end{array}$ & $\begin{array}{l}1800 \pm \\
21\end{array}$ & $\begin{array}{l}44^{\circ} \\
\pm 0^{\circ}\end{array}$ \\
\hline Below $(\mathrm{N})$ & $\begin{array}{l}1704 \pm \\
16 \\
\end{array}$ & $\begin{array}{l}34^{\circ} \\
\pm 0^{\circ} \\
\end{array}$ & $\begin{array}{l}1922 \pm \\
86\end{array}$ & $\begin{array}{l}44^{\circ} \\
\pm 1^{\circ} \\
\end{array}$ & $\begin{array}{l}1767 \pm \\
91\end{array}$ & $\begin{array}{l}43^{\circ} \\
\pm 1^{\circ} \\
\end{array}$ \\
\hline $\operatorname{JRF}_{\text {Shoulder }}(\mathrm{N})$ & $\begin{array}{l}1793 \pm \\
101\end{array}$ & $\begin{array}{l}36^{\circ} \\
\pm 2^{\circ} \\
\end{array}$ & $\begin{array}{l}1942 \pm \\
190\end{array}$ & $\begin{array}{l}44^{\circ} \\
\pm 3^{\circ} \\
\end{array}$ & $\begin{array}{l}1884 \pm \\
166\end{array}$ & $\begin{array}{l}44^{\circ} \\
\pm 2^{\circ}\end{array}$ \\
\hline \multicolumn{7}{|l|}{$n=27$} \\
\hline$\overline{\text { Mean } \pm S D(\mathrm{~N})}$ & $\begin{array}{l}187 \\
\pm 16 \\
\end{array}$ & $\begin{array}{l}41^{\circ} \\
\pm 4^{\circ}\end{array}$ & & & & \\
\hline
\end{tabular}

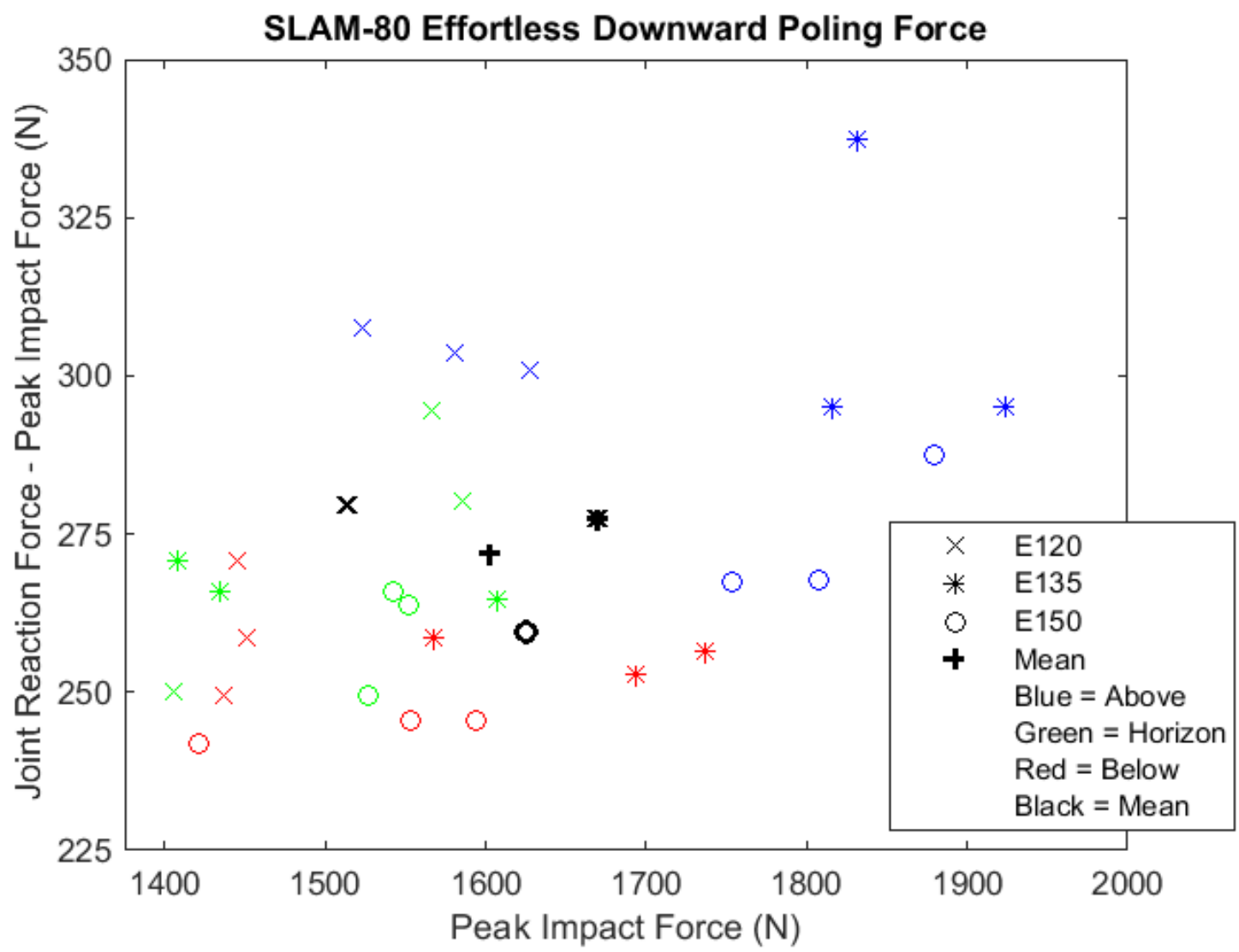

Figure 3.6 SLAM - 80 peak impact force from free fall downward poling against the difference of $J R F_{\text {Shoulder }}$ and peak impact force for all elbow angles $\left(E 120^{\circ}-\mathrm{x}, E 135^{\circ}-^{*}\right.$, and $\left.E 150^{\circ}-0\right)$, and drop heights (above - blue, horizon - green, below - red). Mean values for each elbow angle are represented in black, and mean of all trials (black +). Positive values represent an increase of force at the point of rotation caused from peak impact. 


\subsubsection{Performance and Potential Injury Indicators for Skating in Sledge Hockey}

In sports, torque and momentum can provide key information with regards to the movement under investigation, specifically, performance enhancement and injury prevention. Torque defines the rotational force of an object about a pivot point or axis. Specifically, torque produced from skating in sledge hockey defines the amount of force required through the upper limbs to propel the body and sledge forward. Increase in torque is predicted to result in an increase in explosive skating abilities. However, an increase in torque is also predicted to result in an increase for potential shoulder injury. Positive torque is represented by flexion of the shoulder, opposite the downward stroking motion.

From Table 3.9, torque for all elbow angles was determined to be $1145 \mathrm{Nm} \pm 119 \mathrm{Nm}$. A flexed elbow produces the least amount of torque about the shoulder $(1036 \mathrm{Nm} \pm$ $56 \mathrm{Nm}$ ) compared to all other elbow angles. A mid elbow produces the greatest amount of torque about the shoulder $(1241 \mathrm{Nm} \pm 113 \mathrm{Nm})$, which is slightly different than the extended elbow $(1159 \mathrm{Nm} \pm 81 \mathrm{Nm})$. Investigating extended elbow drop height results, the above drop height produces approximately $140-160 \mathrm{Nm}$ more torque about the shoulder than the remaining two drop heights.

Table 3.9 Estimated torque produced from $S L A M-80$ at peak impact force

\begin{tabular}{|c|c|c|c|}
\hline$n=3$ per condition & $E 120^{\circ}$ & $E 135^{\circ}$ & $E 150^{\circ}$ \\
\hline Above $(\mathrm{Nm})$ & $1086 \pm 23$ & $1372 \pm 33$ & $1260 \pm 29$ \\
\hline Horizon (Nm) & $1042 \pm 59$ & $1130 \pm 50$ & $1122 \pm 13$ \\
\hline Below (Nm) & $981 \pm 7$ & $1221 \pm 48$ & $1096 \pm 45$ \\
\hline TorqueShoulder (Nm) & $1036 \pm 56$ & $1241 \pm 113$ & $1159 \pm 81$ \\
\hline \multicolumn{4}{|l|}{$\mathbf{n}=\mathbf{2 7}$} \\
\hline Mean $\pm \boldsymbol{S D}(\mathrm{Nm})$ & $1145 \pm 119$ & & \\
\hline
\end{tabular}


From Appendix A.3, the change in momentum is defined by impulse. Impulse is defined as the rate of change of an object, and is calculated by the area under a force curve. Specifically, impulse is derived from total stick contact against the force plate $\left(\left[t_{\text {Impact }}: t_{\text {Recoil }}\right]\right)$ and the respective impact forces. In Study 1 , impulse only represents initial impact as the sledge does not move. Increase in impulse is predicted to result in an increase of force transfer into forward skating. Increased impulse should lead to increased momentum of the skater as mass is constant. As skating is a continuous motion, increase in momentum is also predicted to result in an increase in overall skating skill. From Table 3.10, an extended elbow produces the greatest impulse ( $4.4 \mathrm{Ns}$ from $E 150^{\circ}$ trial one) compared to all other elbow angles. The extended elbow also produces the least mean impact impulse with the largest range (3.6 Ns $\pm 0.5 \mathrm{Ns})$. Investigating extended elbow drop height results, the above drop height produces an increase of approximately 1 Ns in impulse than compared to the remaining two drop heights. Overall, there is no difference in lever arm (i.e., elbow angle) and impulse.

Results are aggregated and presented as horizontal impulse for total pick contact against torque at peak impact force (Figure 3.7). From this, a visual representation of lever arm (i.e., elbow angle), drop height, torque, and horizontal impulse are presented allowing a heightened understanding of skating initiation in sledge hockey. From Figure 3.7, on average, an increase in torque is proportional to an increase in horizontal impulse. The flexed elbow (x) produces the least amount of torque with low to mid ranging horizontal impulses compared to the other elbow angles. From an injury prevention aspect, this 
information can be vital. As drop height decreases (blue $>$ green $>$ red), a proportional decrease in torque and horizontal impulse also occurs.

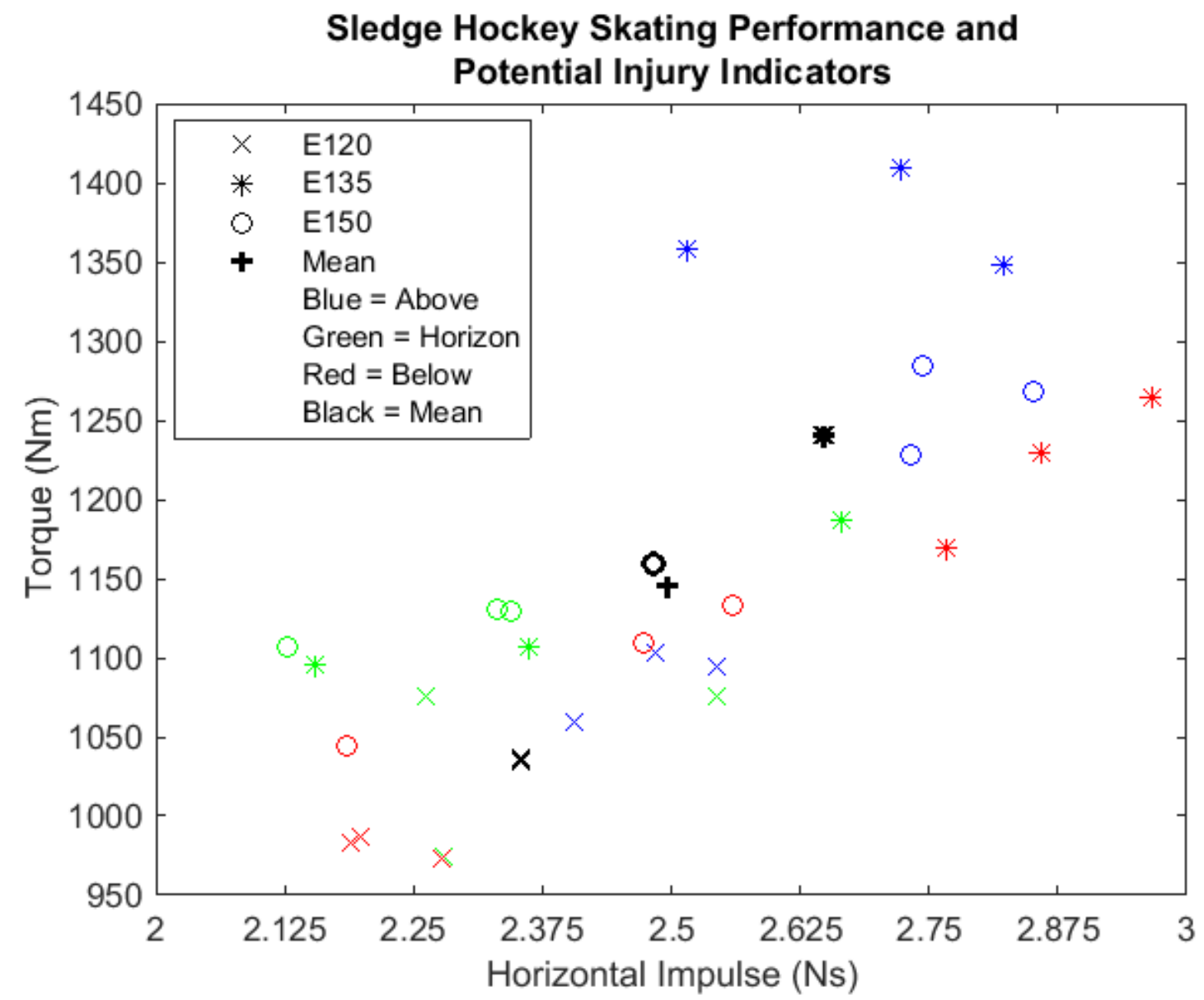

Figure 3.7 SLAM - 80 horizontal impulse against estimated torque about the shoulder for all elbow angles $\left(E 120^{\circ}-\mathrm{x}, E 135^{\circ}\right.$ - *$^{*}$ and $E 150^{\circ}$ - o) and drop heights (above - blue, horizon - green, below red). Mean values for all elbow angles are represented in black, and mean of all trials (black +). Positive torque represents flexion of the shoulder. 
Table 3.10 Impulse for total force plate contact produced by $S L A M-80$ free fall downward poling

\begin{tabular}{lllll}
\hline $\mathbf{n}=$ 3 per condition & $\boldsymbol{E 1 2 0}^{\circ}$ & $\boldsymbol{E 1 3 5}^{\circ}$ & $\boldsymbol{E 1 5 0}^{\circ}$ & Mean \\
\hline Above & & & & \\
Peak Impact Impulse (Ns) & 4.2 & 4.3 & 4.4 & $\mathbf{4 . 3}$ \\
$\begin{array}{l}\text { Mean Impact Impulse (Ns) } \\
\text { Horizon }\end{array}$ & $4.2 \pm 0.05$ & $4.1 \pm 0.28$ & $4.2 \pm 0.13$ & $\mathbf{4 . 2} \pm \mathbf{0 . 1 5}$ \\
Peak Impact Impulse (Ns) & 4.2 & 4.0 & 3.4 & $\mathbf{3 . 9}$ \\
Mean Impact Impulse (Ns) & $4.0 \pm 0.13$ & $3.5 \pm 0.42$ & $3.3 \pm 0.16$ & $\mathbf{3 . 6} \pm \mathbf{0 . 2 4}$ \\
Below & & & & \\
Peak Impact Impulse (Ns) & 3.7 & 4.2 & 3.6 & $\mathbf{3 . 8}$ \\
Mean Impact Impulse (Ns) & $3.7 \pm 0.05$ & $4.0 \pm 0.16$ & $3.4 \pm 0.25$ & $\mathbf{3 . 8} \pm \mathbf{0 . 1 5}$ \\
\hline Peak Impact Impulse (Ns) & $\mathbf{4 . 2}$ & $\mathbf{4 . 3}$ & $\mathbf{4 . 4}$ & \\
Mean Impact Impulse (Ns) & $\mathbf{4 . 0} \pm \mathbf{0 . 2 4}$ & $\mathbf{3 . 9} \pm \mathbf{0 . 3 8}$ & $\mathbf{3 . 6} \pm \mathbf{0 . 4 7}$ & \\
\hline n= 27 & & & & \\
\hline Peak Impact Impulse (Ns) & 4.4 & & & \\
Mean Impact Impulse (Ns) & $3.8 \pm 0.36$ & & & \\
\hline
\end{tabular}

\subsubsection{Estimated Limb Segment Peak Power invloving the Shoulder for Free Fall}

\section{Downward Poling in Sledge Hockey}

From Section 2.2.2, biomechanists use variations of power to evaluate motion. Specifically, total segment power (summation of the power produced by each segment at its respective joint, aggregated as total power of the mechanical system) is used to evaluate the amount of effort required to produce the task. In sledge hockey, the upper limb segment produces motion; therefore, limb segment power provides the rate at which motion occurs. Negative power is understood as extension of the upper limb segment.

From Table 3.11, upper limb segment peak instantaneous power (peak power) for all elbow angles was determined to be $-3035 \mathrm{~W} \pm 433 \mathrm{~W}$. Unlike other kinetic measures, power is directly affected by duration of task. Mean drop time $\left(\left[t_{0}: t_{\text {Peak }}\right]\right)$ was $0.65 \mathrm{~s} \pm 0.12 \mathrm{~s}$, with the flexed elbow angle producing the longest drop time of $0.80 \mathrm{~s}$ (above), and the extended elbow the shortest drop time of $0.43 \mathrm{~s}$ (below). Investigating extended elbow 
drop height results, the above drop height produces approximately $483 \mathrm{~W}$ and $697 \mathrm{~W}$ more negative (extension) power than compared to the horizon and below drop heights, respectively.

Table 3.11 Estimated limb segment peak power produced from $S L A M-80$ at peak impact

\begin{tabular}{llll}
\hline $\mathbf{n}=\mathbf{9}$ per condition & \multicolumn{1}{c}{$\boldsymbol{E 1 2 0}^{\circ}$} & \multicolumn{1}{c}{$\boldsymbol{E 1 3 5}^{\circ}$} & \multicolumn{1}{c}{$\boldsymbol{E} \mathbf{1 5 0}^{\circ}$} \\
\cline { 2 - 3 } Above $(W)$ & $-3082 \pm 121$ & $-3924 \pm 99$ & $-3397 \pm 320$ \\
Horizon $(W)$ & $-2948 \pm 262$ & $-2860 \pm 201$ & $-2914 \pm 164$ \\
Below $(W)$ & $-2465 \pm 111$ & $-3021 \pm 206$ & $-2700 \pm 35$ \\
\hline \hline Mean $\pm \boldsymbol{S D}(\mathbf{W})$ & $\mathbf{- 2 8 3 2} \pm \mathbf{3 2 1}$ & $\mathbf{- 3 2 6 8} \pm \mathbf{5 2 0}$ & $\mathbf{- 3 0 0 3} \pm \mathbf{3 5 8}$ \\
\hline $\mathbf{n = 2 7}$ & & & \\
\hline \hline Mean $\pm \boldsymbol{S D}(\mathbf{W})$ & $\mathbf{- 3 0 3 5} \pm \mathbf{4 3 3}$ & &
\end{tabular}

Specific power (power to mass ratio) allows for comparison between participants. Since no human is exactly the same, specific power is an ideal way to investigate the differences between the same work produced by different people (e.g., [31], [110]). From Table 3.12, specific power per bodyweight $(80 \mathrm{~kg})$ was determined to be $-38 \mathrm{~W} / \mathrm{kg} \pm 1 \mathrm{~W} / \mathrm{kg}$. A mid elbow produces approximately $3 \mathrm{~W} / \mathrm{kg}$ increase during limb extension when creating a free fall downward pole in sledge hockey than an extended elbow. An extended elbow produces approximately $3 \mathrm{~W} / \mathrm{kg}$ increase during limb extension when creating a free fall downward pole in sledge hockey than a flexed elbow. Therefore, a mid elbow requires the most amount of power to produce a free fall downward pole in sledge hockey.

Table 3.12 Estimated limb segment peak specific power produced from $S L A M-80$ at peak impact, normalized by body mass

\begin{tabular}{llll}
\hline $\mathbf{n}=\mathbf{9}$ per $\boldsymbol{E}$ & $\mathbf{E 1 2 0}^{\circ}$ & $\mathbf{E 1 3 5}^{\circ}$ & $\mathbf{E 1 5 0}^{\circ}$ \\
\hline \hline Mean $(\mathrm{W} / \mathrm{kg})$ & $-35 \pm 0.7$ & $-41 \pm 1.2$ & $-38 \pm 0.8$ \\
\hline \hline
\end{tabular}

$\mathbf{n}=\mathbf{2 7}$

Mean $\pm S D(\mathrm{~W} / \mathrm{kg}) \quad-38 \pm 1.0$ 


\subsection{Discussion}

In order to move the body and sledge forward, reaction forces are required in the opposite direction (Newton's third Law). These reaction forces push against the arm and stick causing it to rotate in the other direction. Instead of the arm and stick returning to start as suggested by the pendulum theory, the body and sledge move forward. Visualizing the pick of the stick as a fixed point with the remainder of the limb held static, forward movement of the body occurs due to shoulder extension as the body moves past the point of rotation (shoulder).

From a biomechanical perspective, $S L A M-80$ results suggest that the average male sledge hockey player can produce roughly 2.0 times bodyweight $(80 \mathrm{~kg})$ of impact force per arm, from a free fall downward poling stroke. Specifically, each limb can produce 30 times its weight (5.48 kg) of impact force per stroke initiation. Comparing $J R F_{\text {Shoulder }}$ and impact force, it can be deduced that an increase in force occurs at the shoulder as a result of skating impact. From this, the potential for injury as a result of repetitive surface-stick contact is likely. Potential injuries would be parallel to similarly overloaded weight-bearing injuries seen in running, climbing stairs, and standing up [26], [27].

From Section 2.1.3, peak impact force produced during free fall downward poling in sledge hockey is 4.1 times greater than the maximal force observed by Stoggl and Holmberg during elite upright double poling (475 N) [34]. To the best of the author's knowledge, Stoggl and Holmberg's pole force sampling frequencies were $\geq 2000 \mathrm{~Hz}$, and the signals were unfiltered [34]. From this, the suggestion that skating in sledge hockey does in fact 
introduce substantially increased reaction forces compared to upright poling at force transfer initiation is provided. From this, it can be inferred that preventative and rehabilitation measures can be improved for the shoulder-dependent population by focusing on decreasing risk of overloading the locomotor joints. Further investigation concerning the difference between initial impact forces caused by stick contact between skating in sledge hockey and upright double poling occurs in Chapter 5.

Theoretically, propulsive stick angles should decrease the potential for overloading injuries in sledge hockey as more force should occur in a horizontal direction. From Study 1 results, there was little difference between impact force and elbow angle. By aggregating results via pick angle, further inferences can be made to provide heightened performance and injury prevention information. During Study 1, upon impact, the extended elbow allowed for a slightly greater force transfer when initiating forward locomotion. Specifically, in the extended elbow position two distinct pick impact angles were observed. For the extended elbow, the horizon and below drop heights produced the most propulsive pick impact angles $\left(\sim 35^{\circ}\right)$, where the above and all mid elbow trials produced similar pick impact angles $\left(\sim 40^{\circ}\right)$. From this, the importance of stroke mechanics (extending the elbow) can be predicted as a key factor during initial impact to create skating in sledge hockey. This information can then be interpreted to assist in improving injury prevention involving the load-bearing limbs.

Mechanically, stroke initiation should occur above the horizon ending with a mid to extended forearm. Increasing start height increases the potential for impact force 
(expected), in return increasing related kinetics. Focusing on extended elbow horizon and below drop heights ( $5^{\circ}$ more propulsive stick angle compared to the above drop height), $-287 \mathrm{~N}$ to $-320 \mathrm{~N}$ less force can be redirected back onto the shoulder as a result of $-155 \mathrm{~N}$ to $-235 \mathrm{~N}$ lower impact forces. These lower impact forces then result in, $-138 \mathrm{Nm}$ to $-164 \mathrm{Nm}$ less torque about the shoulder, and $-6 \mathrm{~W} / \mathrm{kg}$ to $-9 \mathrm{~W} / \mathrm{kg}$ less specific power per limb during extension. As previously mentioned, extended above drop height pick impact angles are similar to all mid elbow angles. Similarly, mid elbow and extended elbow results are more closely related than with the flexed elbow results. As the extended elbow produced the most propulsive stick angle and the least amount of increased force at the shoulder from impact, extending the arm during downward poling for skating in sledge hockey can be interpreted as an injury preventive stroke mechanic.

There was a small difference between mid and extended lever arm (i.e., elbow angle) and torque. A small difference in torque also occurred between the extended and flexed elbow, with the flexed elbow requiring the least amount of torque for skating initiation in sledge hockey. Interpretation of results suggest that extending the elbow at lower stroke initiation heights may prove to be an appropriate stroke mechanic, which could potentially decrease the risk of torque introduced injury. Mid and extended elbows produce more power during limb extension than compared to the flexed elbow. From Table 3.5 and Table 3.11, efficiency in producing forward locomotion from the limb segment during the downward pole can be examined. Horizontal impact force (propulsive force) over limb segment power (limb downward stroking power) indicates extending the arm is slightly more efficient $(0.02 \mathrm{~N} / \mathrm{W})$ than a flexed elbow when producing free fall skating initiation in sledge 
hockey.

Rate of force development provides a different perspective when analyzing impact forces. Due to the short duration of the contact phase and elevated impact force, rate of force development is presented in kiloNewtons $(\mathrm{kN})$. Pick-surface contact trial length ranged from $4.0 \mathrm{~ms}$ to $5.0 \mathrm{~ms}$ with an average of $4.3 \mathrm{~ms}$ for all elbow angles. Since sledge hockey is a high-velocity sport with short explosive bursts, rate of force development could prove vital to understanding optimal force transfer positioning. On average, an extended elbow promoted the highest rate of force development to peak force $(757 \mathrm{kN} / \mathrm{s} \pm$ $96 \mathrm{kN} / \mathrm{s}$ ), a $185 \mathrm{kN} / \mathrm{s}$ greater rate than a flexed elbow, and a $174 \mathrm{kN} / \mathrm{s}$ greater rate than a mid elbow.

As in any load-bearing exercise, increased effort and torque are not necessarily a positive for decreasing potential for injury. Increased torque increases stresses against the highly mobile shoulder joint, which may decrease stability within the sole locomotor for shoulderdependent populations (see Section 2.2.2). Chapter 5 continues to discuss lever arm (i.e., elbow angle) and enhanced performance while improving joint protection for sledge hockey players.

\subsubsection{SLAM - 80 and Future Research Involving Skating in Sledge Hockey}

From Study 1, SLAM - 80 could be used in lieu of sledge hockey players to evaluate impact forces and their respective kinetics. Study 2 will be used to validate $S L A M-80$ as a reliable mechanical system for skating initiation in sledge hockey (Chapter 5). From this, 
overuse and overloading of the locomotors can be investigated without exposing human subjects to additional stresses onto their bodies. Variations of the prototype can be made enhancing the understanding of potential injury caused by stick impact (e.g., body composition, segment size, joint angles, etc.).

From a performance enhancing viewpoint, mid to extended elbow pick impact angles $\left(33^{\circ}-42^{\circ}\right)$ fall within the range outlined by all double poling contact angles $\left(12^{\circ}-84^{\circ}\right)$ [24], [34], [42], [44], [45], [50], [54]. A similarity is observed within Nilsson et al. peak impact angles for various upright pole lengths $\left(42^{\circ}-45^{\circ}\right)$ [50]. From simple vector properties, vector magnitude (pole length) and vector direction (pole angle) are independent of each other (angle of peak force transfer is identical throughout the length of the pole). From this, in conjunction with sport-specific acromial height, mid and extended arms (longer lever arm) should promote increased forward propulsion. Conclusions cannot be drawn regarding forward locomotion as all outlined results provide information regarding force transfer initiation (the sledge did not move). Musculoskeletal data are required to fully understand forward locomotion in the sport of sledge hockey (e.g., push-off reaction forces). Further investigation supporting the use of $S L A M-80$ in lieu of human participants is provided in Chapter 5.

\subsubsection{Limitations}

Limitations observed during Study 1 include:

1. Sagittal plane computation limits the overall understanding of a motion when the major contributor is a tri-axial joint moving in a cyclical pattern (the $G H J$ cannot 
rotate continuously for $360^{\circ}$, ab/adduction is inevitable to complete the cycle). Moreover, as an arm moves down and inward, only the downward motion is tracked. The movement inwards has a slight effect on the co-ordinates true locations. Tri-axial analysis is recommended when possible to assist in a full understanding of skating in sledge hockey.

2. The design allowing absorption at the elbow and wrist-pick provided a more dynamic joint than intended, especially after so many testing sessions (see Section 7.2). Larger screws or hole filler is recommended to return the axel hole to the original snug fit.

3. Positioning within the capture area, marker location, and/or MOCAP calibration error can cause errors in data measurement, even when care with setup and calibration procedures are conducted. A slight adjustment with SLAM80 alignment with respect to MOCAP global co-ordinates will help minimize measurement error in uniplanar analysis. Due to the close proximity of the dynamic limb to the edge of the sledge, $S L A M-80$ could not be perfectly parallel with the global x-axis.

4. Anterior-downward displacement observed at the shoulder joint-centre (Figure 3.4) was determined to have been caused by the narrow structural design and lack of weight associated with the trunk of $S L A M-80$. Shoulder joint-centre displacement for all trials within this study was determined to be $2.05 \mathrm{~cm} \pm$ $0.17 \mathrm{~cm}$, with all trials displaying similar displacement.

5. Release time of the Velcro strap had a slight effect on total trial length (human error). A standardized rigging system is recommended to ensure equal release 
during future investigation.

\subsection{Conclusion}

From a sport-specific viewpoint, with a torso flexion of $40^{\circ}$, forearm extension produces elevated force transfer during a free fall downward pole in the sport of sledge hockey. Stroke initiation producing enhanced forward locomotion is suggested to start above shoulder level. Pick impact angle range should occur between $33^{\circ}-42^{\circ}$ with a mid to extended elbow. A free fall extended elbow produces less power during limb extension and more torque about the shoulder than a flexed elbow. However, the extended elbow is slightly more efficient than the flexed elbow during a downward free fall stroke, and produces the least increase in force at the shoulder. Proper mechanics within the stroking cycle, specifically at contact, should decrease the potential for overuse/overloading injuries caused from skating in sledge hockey.

Pure mathematical models (e.g., computer simulations) do not always represent the truest results, specifically concerning power required for effortless downward poling. During applied experiments unknown and unpredictable changes may occur within the experiment (random error). An example of random error causing discrepancies when comparing estimated results to pure mathematical results may include the absorption properties of the elbow and wrist.

In conclusion, baseline measures for each drop height, elbow angle, and as a combination are determined for $S L A M-80$. These data represent non-contractile produced kinetics, which are used to improve analysis of musculoskeletal produced kinetics outlined then discussed in Chapter 5. 


\section{Chapter: Study 2: Task Naïve Off-Ice Skating in Sledge Hockey}

This chapter consists of seven sections. Section 4.1 outlines the rationale for supporting the use of a task naïve population to identify key areas of difficulty during skating in sledge hockey; specifically, areas of difficulty observed during the propulsion phase.

Section 4.2 provides the methodology used during human participant data collection. Section 4.2.4 outlines participant preparation implemented prior to testing.

Section 4.3 provides information regarding the equipment and apparatuses used in Study 2. Section 4.3.1 outlines the modifications made to the on-ice sledge and sticks. Section 4.3.2 outlines how the elbow and wrist angles for participants were restricted. Sections 4.3.3, 4.3.4, and 4.3.5 outline the MOCAP - force plate, and $S E M G$ systems used during data acquisition, respectively. Section 4.3.6 outlines data acquisition and processing specifics used for analysis.

Section 4.4 outlines the testing protocol defining each task required to be performed.

Section 4.5 provides the results from each participant and as a tested population mean for skating in sledge hockey off-ice. Section 4.5.1 provides sagittal plane trajectory interpretations specifically concerning pick contact angles. Section 4.5 .2 provides contact force analysis specifically concerning peak impact force and push-off force. Section 4.5.3 provides the estimated $J R F_{\text {Shoulder }}$ and its interpretations specifically concerning estimated peak $J R F_{\text {Shoulder }}$. Section 4.5.4 provides information regarding three key 
measures for performance and injury prevention analysis. This section provides the estimated torque about the shoulder, momentum calculated from pick force plate contact and the respective reaction forces, and body-sledge velocity. Section 4.5 .5 provides the estimated limb segment power at the shoulder and its interpretations specifically concerning estimated peak power and specific power.

Sections 4.6 discusses analogies made from Study 2 acquired results in regards to skating in sledge hockey, and the potential for injury as a result of the propulsion phase. Support is provided identifying increased impact force at initial contact as mechanism for potential injury.

Section 4.7 summarizes the results providing conclusions for preliminary interpretations involving skating in sledge hockey.

\subsection{Task Naïve Population and Sledge Hockey Skating Analysis}

An able-bodied naïve (little to no experience) population preforming a skill/sport typically enjoyed by $P w D s$ can act as a pilot population enabling research methodologies to be finetuned, ideally allowing for smoother testing sessions involving participants with unique needs/requirements $(P w D s)$. Simultaneously, a naïve population can provide a unique viewpoint with truer representation of the difficulties endured during a task, if they exist. Study specific, naïve sledge hockey players can provide insight to the difficulties endured while skating in sledge hockey, off-ice, if they exist. Information acquired can indicate areas of difficulty, which may not be present in skilled participants improving the 
understanding of the motion. Interpretations concerning areas of difficultly can then be directed towards coaching, preventative, and rehabilitative practices improving quality of life for the shoulder-dependent population.

In contrast, a naïve population does not perform tasks in the same manner as an expert population. This difference in task performance was observed in the study conducted by Stoggl et al. outlined in Section 2.1.4 [45]. As skill improved, so did the strategies used to perform the task (performance was more efficient) [45]. Since the tested population for Study 2 involves task naïve participants, results do not directly represent the typical sledge hockey player. Instead results offer suggested areas of interest for future investigations involving the elite population. Naturally, the best representation of skating in sledge hockey is from an expert population.

Observable parameters are used to assess motion, specifically gait [35]. Relevant generalized observable parameters for skating in sledge hockey should include: 1) symmetry throughout the bilateral strokes (absence of muscular and/or mobility limb dominance), 2) imbalance (lean, stagger, or fall), 3) fluidity throughout the stroking pattern (independent (unilateral consistent trajectory patterns) and dependent (bilateral consistent trajectory patterns) between the limbs), and 4) positive habituation (improved performance over repeated tasks).

This thesis examines the relationship between limb dominance to skating ability through the use of task naïve participants with various dominant limb (upper, lower, and both) 
athletic abilities, if they exist. Specifically, upper limb and both limb dominant participants are predicted to perform better than the lower limb dominant participant. Performance is ranked from the above-mentioned observable gait parameters with specific focus on positive habituation and imbalance (primarily qualitative analysis).

\subsection{Methodology: Participant Recruitment and Preparation}

This section outlines the methodologies used for Study 2 concerning the participants.

\subsubsection{Research Ethic Boards Approval and Requirements}

Ethical approval was provided from Carleton University's Research Ethics Board (Appendix D.1), and the University of Ottawa's Health Sciences and Science Research Ethics Board (Appendix D.2). All data were collected at the Human Movement Biomechanics Laboratory, Lees Campus - University of Ottawa, Ottawa, Canada. A maximum of 3 hours was allotted per testing session.

\subsubsection{Participant Recruitment}

Task-naïve participants were recruited from Carleton University and University of Ottawa student populations. Body composition (upper torso mesomorph) and athletic ability resembling Paralympians were used as a form of recruitment. If participants were associated with a varsity sports team, coaches were supplied with hardcopies of all required

documents. Participants were presented with a Letter of Invitation (Appendix D.3) upon recruitment. 
Hardcopy packages were provided to each participant including: 1) Ethical Approval Certificates, 2) Letter of Invitation, 3) Participant Procedure (Appendix D.4), 4) Written Consent Form (Appendix D.5), 5) Physical Activity Readiness Questionnaire $(P A R-Q)$ (Appendix D.6) [111], 6) Questionnaire Written Consent Form (Appendix D.7), and 7) Interview Questionnaire (Appendix D.8).

\subsubsection{Anthropometrics and Athletic Abilities}

Participants were five right-handed (handedness was not a selection criterion for participants) able-bodied adult males with an average age of $22.2 \pm 2.2$ years, body mass of $82 \mathrm{~kg} \pm 9.8 \mathrm{~kg}$, and sitting height of $0.92 \mathrm{~m} \pm 0.02 \mathrm{~m}$ (Table 4.1). Sitting height was measured from the base of the sledge to the top of the head minus $0.005 \mathrm{~m}$ for sledge thickness. C4 participated once in the sport of sledge hockey, and all participants had at least played pond hockey. Three participants were active varsity football players (two wide receivers and one linebacker, $C 3$ and $C 5$, and $C 2$ respectively), one recreational soccer player $(C 1)$, and a recreational hockey player $(C 4)$. Participants were recruited for their athletic abilities within their chosen sport in order to provide information regarding limb dominance and athletic ability for naïve skill-level players in the sport of sledge hockey. None of the recruited participants presented expertise in upper limb stroking patterns (antiphase contralateral and/or bilateral) foreshadowing asymmetrical bilateral skating among all participants. 
Table 4.1 Anthropometric measurements and athletic abilities for participants in Study 2

\begin{tabular}{|c|c|c|c|c|c|c|c|c|c|}
\hline & \multirow[t]{2}{*}{$\begin{array}{l}\text { Athletic } \\
\text { Sport of } \\
\text { Focus }\end{array}$} & \multirow[t]{2}{*}{$\begin{array}{l}\text { Athletic } \\
\text { Dominant } \\
\text { Limb } \\
\text { Set(s) } \\
\end{array}$} & \multirow[t]{2}{*}{$\begin{array}{l}\text { Sitting } \\
\text { Height } \\
\text { (m) }\end{array}$} & $\begin{array}{l}\text { Upper Limb } \\
\text { Length (m) }\end{array}$ & $\begin{array}{l}\operatorname{Limb} \\
(\mathrm{m})\end{array}$ & \multicolumn{2}{|c|}{$\begin{array}{l}\text { Upper Arm } \\
\text { Length (m) }\end{array}$} & \multicolumn{2}{|c|}{$\begin{array}{l}\text { Forearm } \\
\text { Length (m) }\end{array}$} \\
\hline & & & & $\mathbf{L}$ & $\mathbf{R}$ & $\mathbf{L}$ & $\mathbf{R}$ & $\mathbf{L}$ & $\mathbf{R}$ \\
\hline$C 1$ & Soccer & Lower & 0.902 & 0.560 & 0.572 & 0.350 & 0.365 & 0.294 & 0.304 \\
\hline$C 2$ & $\begin{array}{l}\text { Line } \\
\text { Backer }\end{array}$ & Upper & 0.919 & 0.571 & 0.587 & 0.356 & 0.366 & 0.310 & 0.310 \\
\hline$C 3$ & $\begin{array}{l}\text { Wide } \\
\text { Receiver }\end{array}$ & Upper & 0.931 & 0.567 & 0.567 & 0.350 & 0.355 & 0.309 & 0.312 \\
\hline$C 4$ & Hockey & Both & 0.912 & 0.523 & 0.528 & 0.324 & 0.315 & 0.275 & 0.274 \\
\hline \multirow[t]{2}{*}{$C 5$} & $\begin{array}{l}\text { Wide } \\
\text { Receiver }\end{array}$ & Upper & 0.957 & 0.529 & 0.535 & 0.329 & 0.331 & 0.295 & 0.300 \\
\hline & & $\begin{array}{r}\text { Mean } \\
\pm \\
S D\end{array}$ & $\begin{array}{l}\mathbf{0 . 9 2 4} \\
\pm \\
0.021\end{array}$ & $\begin{array}{l}0.550 \\
\pm \\
0.022\end{array}$ & $\begin{array}{l}0.558 \\
\pm \\
0.025\end{array}$ & $\begin{array}{l}0.342 \\
\pm \\
0.014\end{array}$ & $\begin{array}{l}0.346 \\
\pm \\
0.023\end{array}$ & $\begin{array}{l}0.297 \\
\pm \\
0.014\end{array}$ & $\begin{array}{l}\mathbf{0 . 3 0 0} \\
\pm \\
\mathbf{0 . 0 1 5}\end{array}$ \\
\hline
\end{tabular}

\subsubsection{Participant Preparation}

All forms of written consent were collected during participant preparation. The $P A R-Q$ and interview questionnaire were completed. $C 3$ required additional approval from an athletic therapist post $P A R-Q$. Participant preparation duration did not exceed an hour. C2 was required to return due to equipment malfunctions; anthropometric data collection was not repeated. C3's preparation was unintentionally interrupted by a fire alarm evacuation increasing the duration of the session. This did not appear to have an effect on the overall physical demands on the participant. Detailed information regarding participant documentation can be found in Appendix D.3 through to Appendix D.8 as outlined in Section 4.2.2. Suggested modifications to any and all forms are outlined in Appendix D

All tests were performed bare-chested. Skin preparation following standard protocols for $s E M G$ electrode placement occurred next [92], [112]-[115]. A synopsis of this protocol 
includes: 1) skin marked location for electrode placement (symmetrical placement), 2) removal of loose or dead epithelial cells (shaved and aggressively rubbed with rubbing alcohol pads), and 3) electrode placement. $s E M G$ electrodes were placed bilaterally on the 1) latissimus dorsi, 2) lower trapezius, 3) posterior deltoid, 4) triceps brachii (longhead), and 5) biceps brachii as recommended by SENIAM and Delsys (Figure 4.1) [112], [113]. C2's left latissimus dorsi was placed over a heavily inked tattoo. Signal distortion was not foreseen to be an issue and the acquired signal did not appear to produce data to implicate additional artifact. Upon investigation of peer-reviewed literature involving biosignals and tattoo ink, no warnings were provided to indicate known artifact. In fact some $s E M G$ electrode sites were tattooed for replacement during longer term studies [116]. Moreover, semi-permanent printed tattoos have become a newly investigated method for improving $s E M G$ electrodes [115]

Marker placement followed using $14 \mathrm{~mm}$ diameter reflective spheres and the University of Ottawa Motion Analysis Model (UOMAM). Markers were placed bilaterally indicating the following musculoskeletal landmarks: 1) front and back head, 2) shoulder, 3) upper arm, 4) elbow, 5) forearm, 6) anterior and posterior wrist, and 7) hallux (Figure 4.1). A right back (scapula) marker is used to distinguish anterior and posterior in upright participants. Typically, this model requires the use of the anterior superior iliac spine $(A S I S)$, however, these landmarks become obstructed during task performance. Bilateral Rib 10 was marked vertically, directly above the ASIS landmark location. or as close to as possible, and the distance between measured (Figure 4.1). Symmetry was not required as not all participants had symmetrical rib cages; locations were marked with ink to ensure 
proper measurement and placement/replacement during testing.

Table 4.2 ASIS to Rib 10 distance for participants in Study 2

\begin{tabular}{lll}
\hline $\mathbf{n}=\mathbf{5}$ & $\begin{array}{l}\text { Left } \boldsymbol{R i b} \mathbf{1 0}-\text { Left } \\
\boldsymbol{A S I S}(\mathbf{m})\end{array}$ & $\begin{array}{l}\text { Right Rib 10 }- \text { Right } \\
\boldsymbol{A S I S}(\mathrm{m})\end{array}$ \\
\hline \hline $\boldsymbol{C 1}$ & 0.125 & 0.130 \\
$\boldsymbol{C 2}$ & 0.130 & 0.131 \\
$\boldsymbol{C} 3$ & 0.122 & 0.106 \\
$\boldsymbol{C 4}$ & 0.125 & 0.126 \\
$\boldsymbol{C 5}$ & 0.125 & 0.115 \\
\hline Mean $\pm \boldsymbol{S D}$ & $\mathbf{0 . 1 2 5} \pm \mathbf{0 . 0 0 3}$ & $\mathbf{0 . 1 2 2} \pm \mathbf{0 . 0 1 1}$ \\
\hline
\end{tabular}




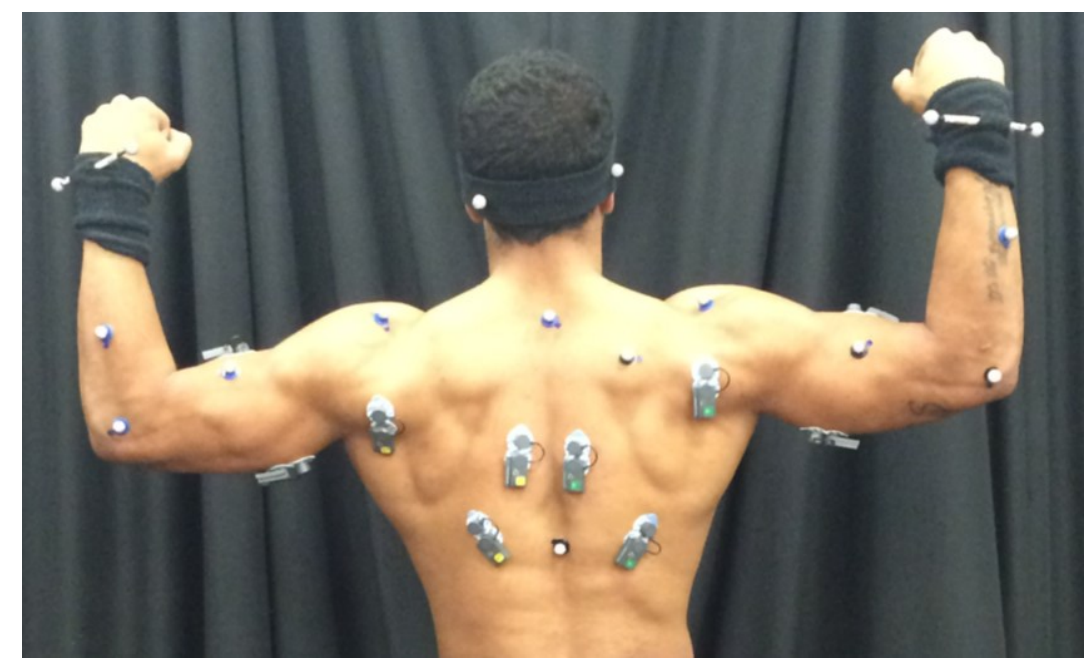

(a)

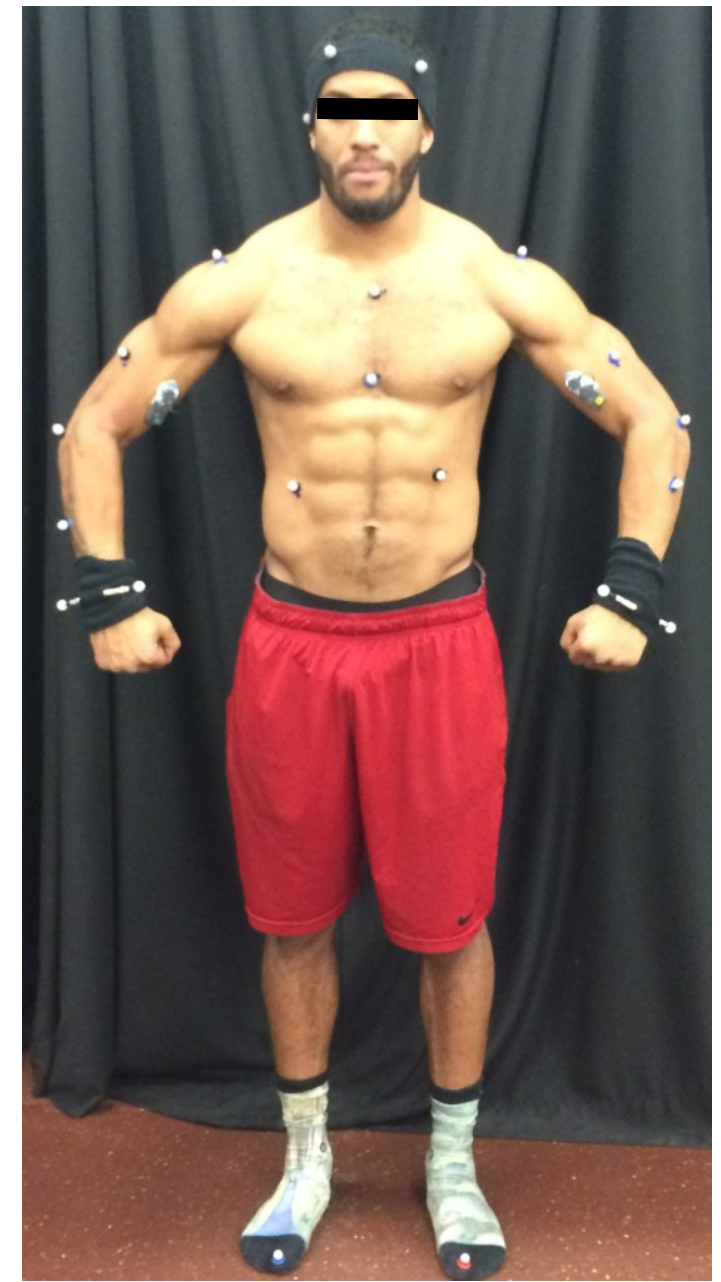

b)

Figure 4.1 sEMG and marker locations for Study 2 as per Section 4.2.4 (a) Posterior, and (b) Anterior views. 


\subsection{Methodology: Equipment and Acquisition Apparatus}

This section outlines the methodologies used for Study 2 concerning the equipment and apparatuses used.

\subsubsection{Off-Ice Modifications to On-Ice Sledge Hockey Equipment}

Sledge hockey is typically an on-ice sport with no known off-ice designed equipment. This study modified on-ice sledge hockey equipment in order to perform indoor off-ice analysis. Modifications support a major limitation seen in biomechanical analysis, which concerns the equipment-environment interface and uncompromised desired motions.

International regulations require a double chassis system to support the bucket of the sledge [8]-[10]. For off-ice analysis, the author replaced both blade-chassis with two youth sized rollerblade wheel-chassis. Two $64 \mathrm{~mm}$ rubber rollerblade wheels were placed in the front most and backmost positions in each chassis. Each wheel was equipped with $A B E C-5$ bearings and tightened to produce a slightly restricted roll; wheel tension was not adjusted post installation. Distance between the chassis mimicked a narrow-blade orientation $(5 \mathrm{~cm}$ from screw-centre to screw-centre, above average skill level in sledge hockey) and was unchanged post installation; expert sledge hockey blade orientation resembles a single blade in order to promote agility [8].

Since most sledges are participant specific, telescopic leg-rests were required to be designed in order for all participants to use the same equipment. Two metal tubes were cut and drilled every $5.08 \mathrm{~cm}$ for approximately $30.48 \mathrm{~cm}$ creating a telescopic leg-rest. Two 
metal slips were cut, drilled, and used to connect the leg-rest to the foot-rest (Figure 4.3). Acknowledgement is made to the author's uncle Marc Fournier for assisting the author in construction of the telescopic leg-rests.

Post initial participant, a single $64 \mathrm{~mm}$ rollerblade wheel was added to the left front of the sledge in order to provide improved balance. Protection of the floor and force plates increased with this addition. For the initial participant ( $C 1$ by random code assignment), the front of the sledge was duct taped with a fabric pad to decrease friction, and wear and tear on the laboratory floor. It was unforeseen that the participants would require the use of the support for additional balance during motion. Typically, the front of the sledge is raised during skating and is only used for balance during rest. Figure 4.2 provides the exploded view combining the telescopic leg rests and additional front wheel modifications. Figure 4.3 depicts the sledge used for all data collection.

A marker was placed at the back middle top of the sledge in order to provide a location for trunk angle analysis; ASIS markers usually provide this location. Additionally, in conjunction with the halluces' markers the sledge is reproducible via computer software. 

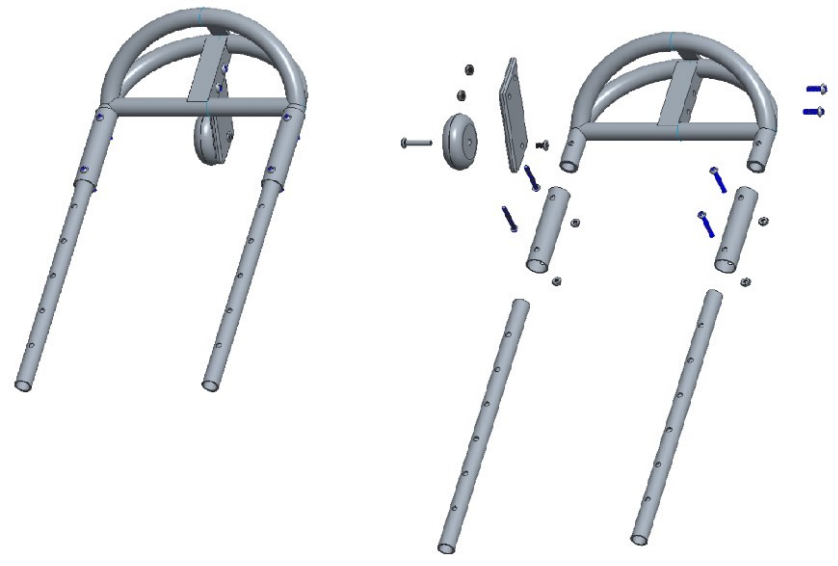

SCALE $\quad 0.250$

Figure 4.2 Exploded view of the telescopic leg-rest and wheel addition designed for multiple participant use during off-ice sledge hockey skating analysis.

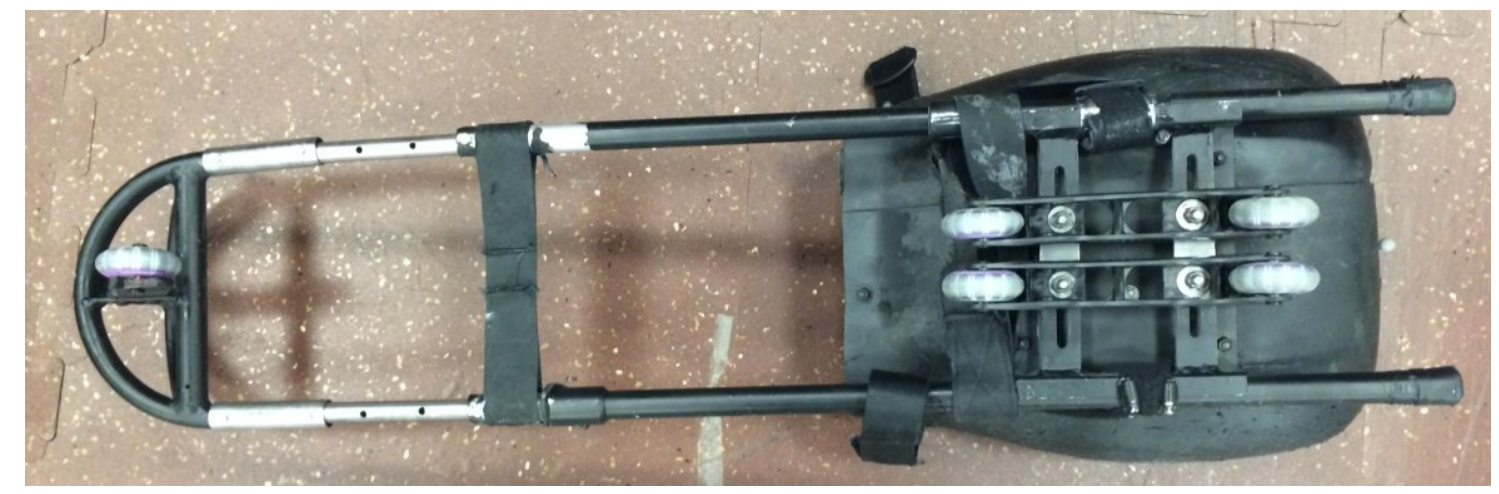

(a)

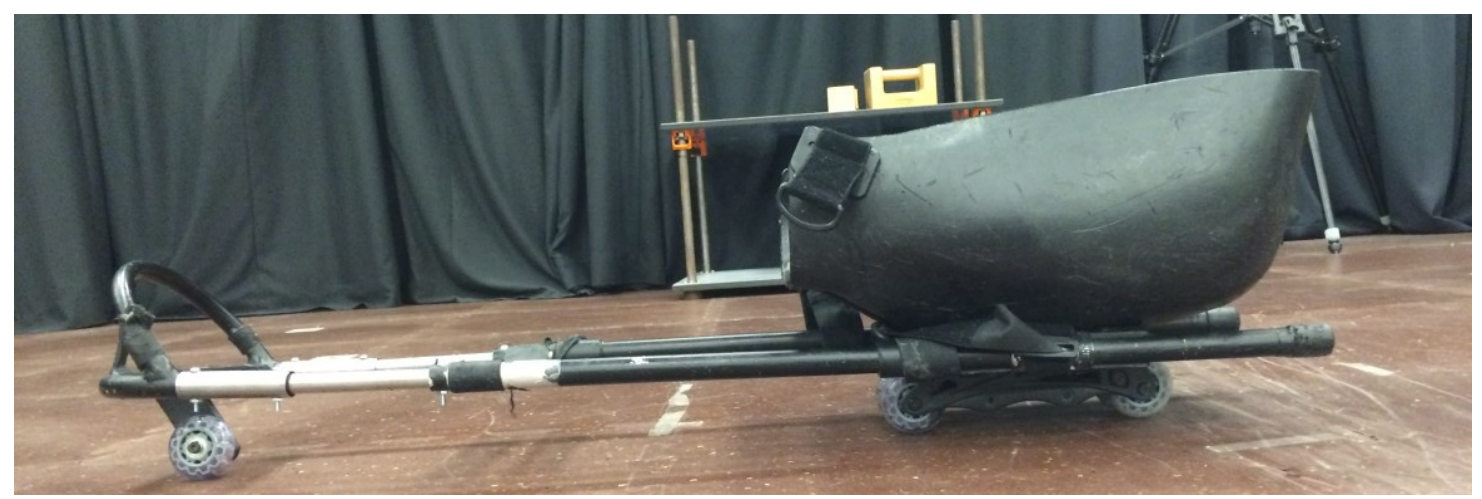

(b)

Figure 4.3 Indoor modified sledge hockey sledge used for skating analysis (a) Wheel configuration, and (b) Sagittal plane view. 
As previously stated in Section 3.3.1, the picks of the sticks were covered with cotton tape in order to reduce the impact of the sharp pick points against the force plates (Figure 4.4). After a few practice trials tape did not affect force transfer during propulsion. Tape was only replaced if determined the pick would damage the force plate. Markers were placed at the blade, joint, and pick of each stick as outlined in Section 3.3.1 and Figure 4.4. Marker placement was performed by two different research assistants creating two distinct marker locations with regards to the sticks' markers. For future research, stick marker locations are suggested to be standardized. As pick marker locations were not at the pick-surface interface $(y=0.000 \mathrm{~m})$, marker locations were measured and recorded to ensure proper pick marker shifting upon data processing (Table 4.3), similar to Study 1. Markers also allowed for stick reproduction via computer software. Figure 4.4 illustrates the sledge hockey sticks used for all data collection. All sledge hockey equipment was spray painted flat black in order to reduce reflection.

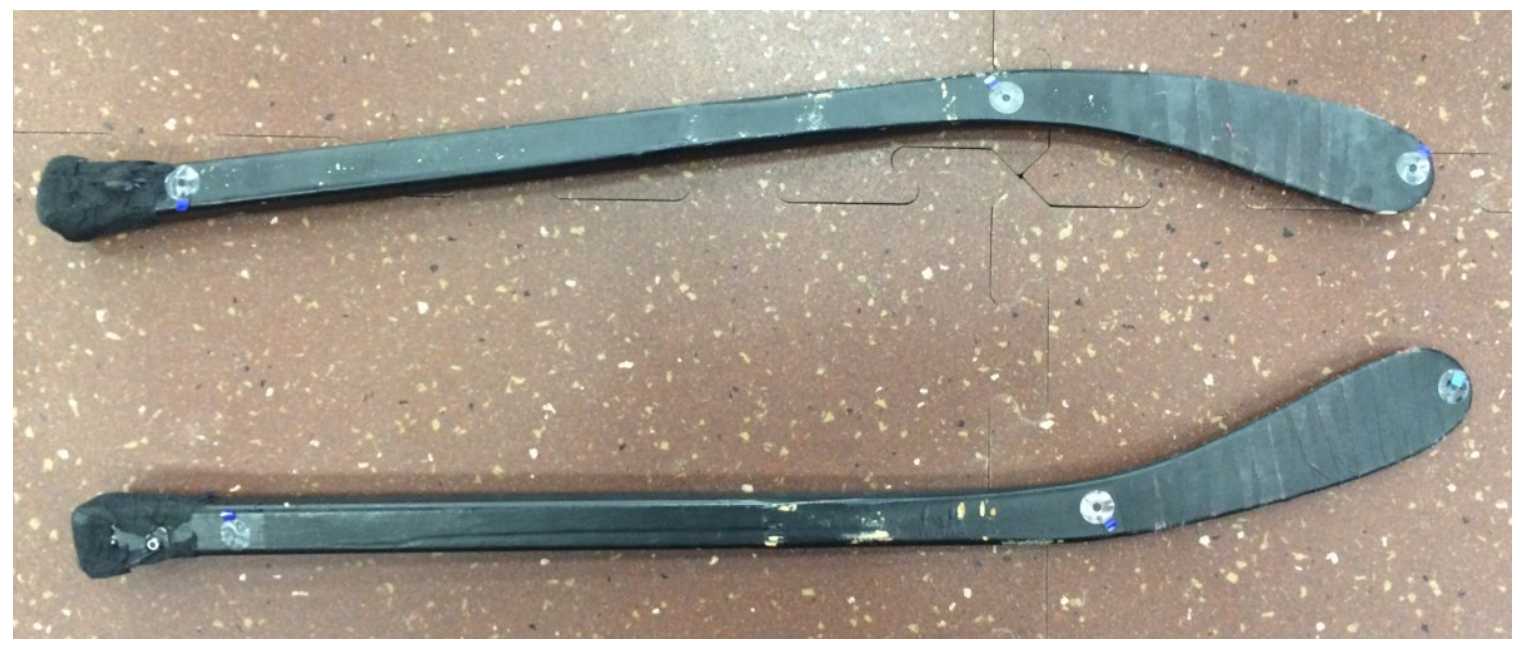

Figure 4.4 Sledge hockey stick used for indoor analysis with general marker locations (shiny circles two-sided adhesive). 
Table 4.3 Stick marker measurements

\begin{tabular}{lllllll}
\hline $\mathbf{n}=\mathbf{5}$ & $\begin{array}{l}\text { Blade Tip to Marker } \\
(\mathbf{m})\end{array}$ & & \multicolumn{2}{l}{$\begin{array}{l}\text { Pick to Joint Marker } \\
(\mathbf{m})\end{array}$} & \multicolumn{2}{l}{$\begin{array}{l}\text { Pick to Pick Marker } \\
(\mathbf{m})\end{array}$} \\
\hline \hline & $\mathbf{L}$ & $\mathbf{R}$ & $\mathbf{L}$ & $\mathbf{R}$ & $\mathbf{L}$ & $\mathbf{R}$ \\
\hline $\boldsymbol{C 1}$ & 0.020 & 0.015 & 0.536 & 0.558 & 0.073 & 0.061 \\
$\boldsymbol{C 2}$ & 0.021 & 0.015 & 0.540 & 0.565 & 0.088 & 0.090 \\
C3 & 0.013 & 0.013 & 0.533 & 0.554 & 0.076 & 0.084 \\
C4 & 0.015 & 0.012 & 0.550 & 0.522 & 0.067 & 0.055 \\
C5 & 0.015 & 0.012 & 0.550 & 0.522 & 0.067 & 0.055 \\
\hline Mean & $\mathbf{0 . 0 1 7} \pm$ & $\mathbf{0 . 0 1 3} \pm$ & $\mathbf{0 . 5 4 2} \pm$ & $\mathbf{0 . 5 4 4} \pm$ & $\mathbf{0 . 0 7 4} \pm$ & $\mathbf{0 . 0 6 9} \pm$ \\
\pm SD & $\mathbf{0 . 0 0 3}$ & $\mathbf{0 . 0 0 2}$ & $\mathbf{0 . 0 0 8}$ & $\mathbf{0 . 0 2 1}$ & $\mathbf{0 . 0 0 9}$ & $\mathbf{0 . 0 1 7}$ \\
\hline
\end{tabular}

\subsubsection{Elbow Brace, Wrist Restriction, and Restricted Angles}

Two LE101 Locking Elbow Orthosis elbow braces were used during restricted-elbow tests (Figure 4.5) [117]. Due to the elbow brace design, desired elbow angle was estimated with a hand-sized protractor prior to being strapped around the participant's arms. Braces allowed slight movement at the elbow. Desired elbow angles reflected $S L A M-80$ 's baselines $\left(120^{\circ}, 135^{\circ}\right.$, and $\left.150^{\circ}\right)$. Simple mathematical angle verification occurred during data processing ensuring a flexed, mid, and extended elbow. Elbow and forearm markers were placed at their estimated location on the braces, if required. Acknowledgment is made towards Dr. Edward Lemaire and colleagues at the Ottawa Hospital Research Institute for the use of the elbow braces.

Wrists were restricted with two wooden tongue depressors, pro-wrap, and duct tape per wrist for selected trials (Figure 4.6). Wrist braces were not used as they interfered with palm-stick interfaces significantly altering the stroking cycle. Wrist flexion was the undesired motion and this restriction-design was determined appropriate for the task at hand. 


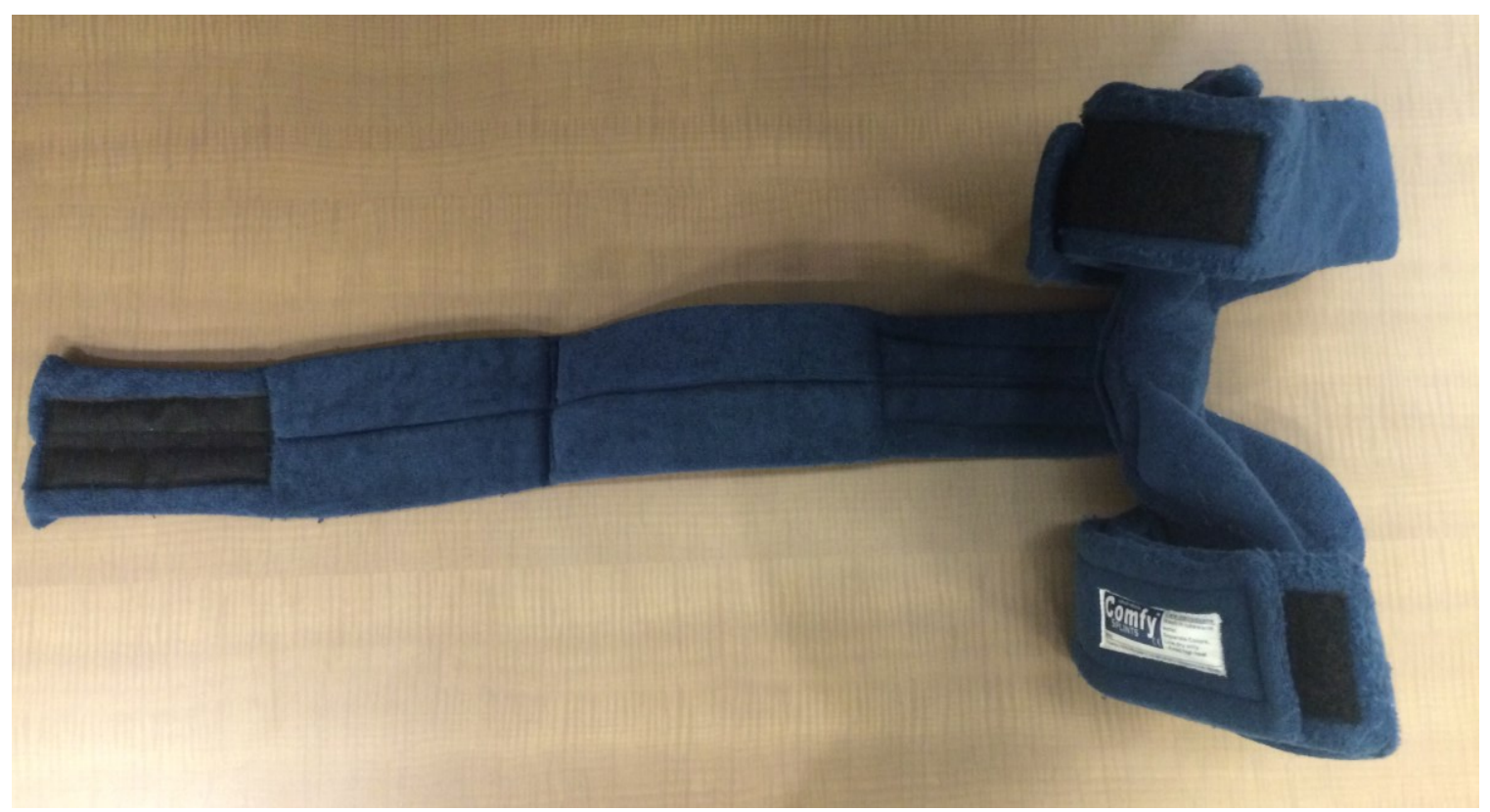

Figure 4.5 LE101 elbow brace used for restricted trials.

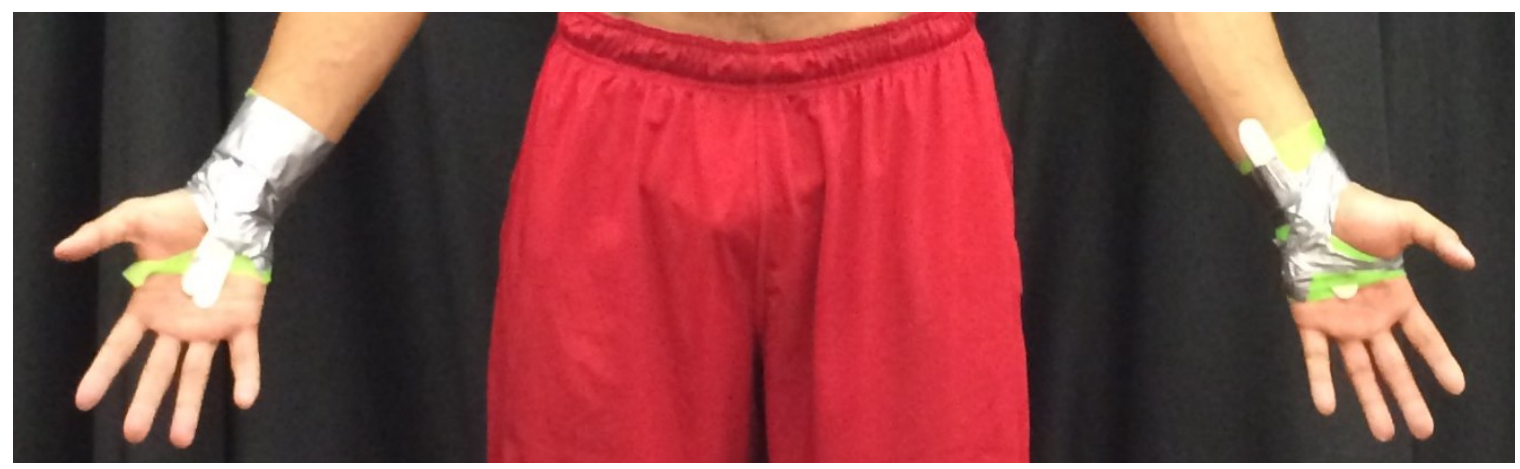

Figure 4.6 Wrist flexion-restricting design for restricted tests promoting appropriate palm-stick interfaces.

\subsubsection{Motion Capture System}

The same Vicon MOCAP system as Study 1 (Section 3.3.1) was used to acquire trajectory data, with Vicon Nexus as the corresponding computer software (Figure 4.7). Trajectory data were acquired at $250 \mathrm{~Hz}$. 


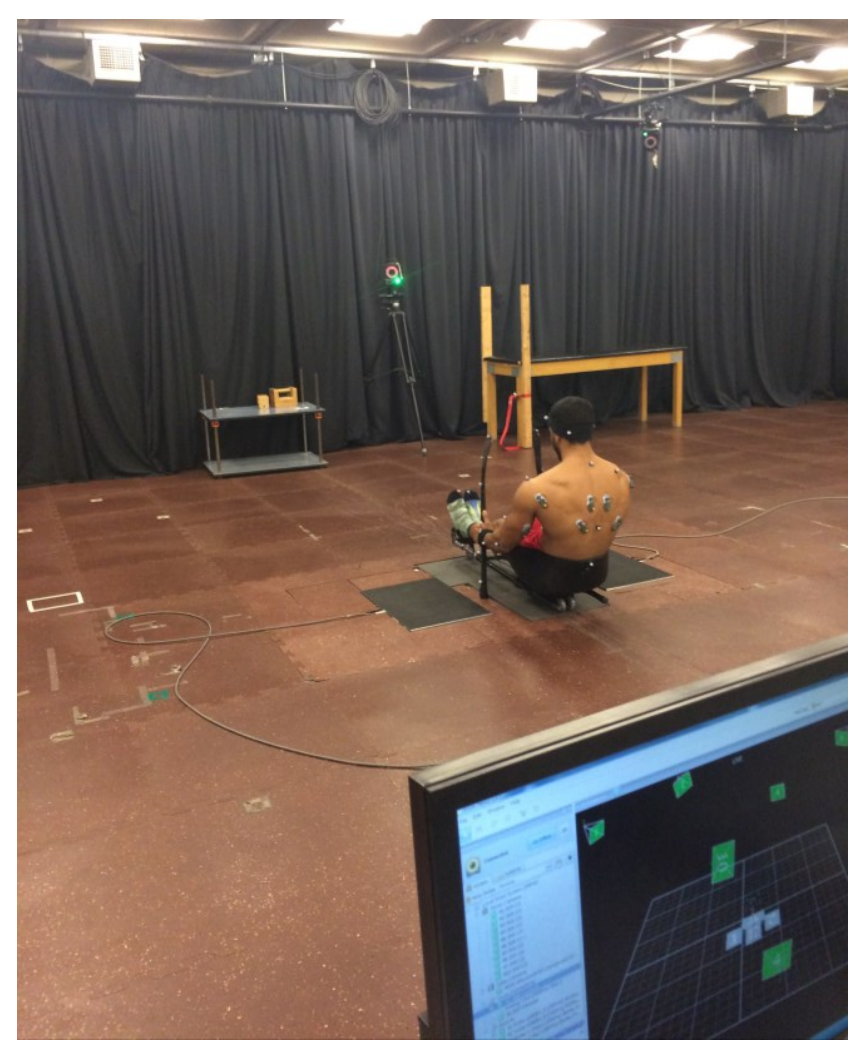

Figure 4.7 Vicon MOCAP system configuration Human Movement Biomechanics Laboratory for a Static Free test trial.

\subsubsection{Force Plate System}

Due to the vast range of physical abilities, a number of para-sports are performed in the seated position. This presents increased difficulty when obtaining GRFs from dynamic movements. All points of contact are required to be in contact with a force plate in order to determine total reaction force. If a force plate acquires more than one point of contact information is integrated eliminating the ability to analyze the individual movement.

The author designed a force plate orientation with the ability to acquire each individual stick, both rear wheel chassis, and the front wheel. This offset ' $t$ ' formation provided the correct orientation in order to simultaneously acquire individual $G R F S$ from the four points 
of contact. Acknowledgement is made to the author's brother Adam N. Gal for assisting the author in construction of the force plate system platform.

Figure 4.8 provides an aerial view of the force plate system used in this study. Left and right Bertec FP4060-08 force plates were lined with the same thin wavy rubber pad used in Study 1, for the same purposes [107]. Two Kistler 9286AA force plates were used in the middle to collect the participant-sledge reaction forces [118]. Participants travelled through the system entering from the length-wise Kistler force plate and exited off the horizontal Kistler force plate with respect to Figure 4.8 (yellow arrow - direction of travel). Force plates were centrally located within the capture zone. All force plate data were collected at $2000 \mathrm{~Hz}$.

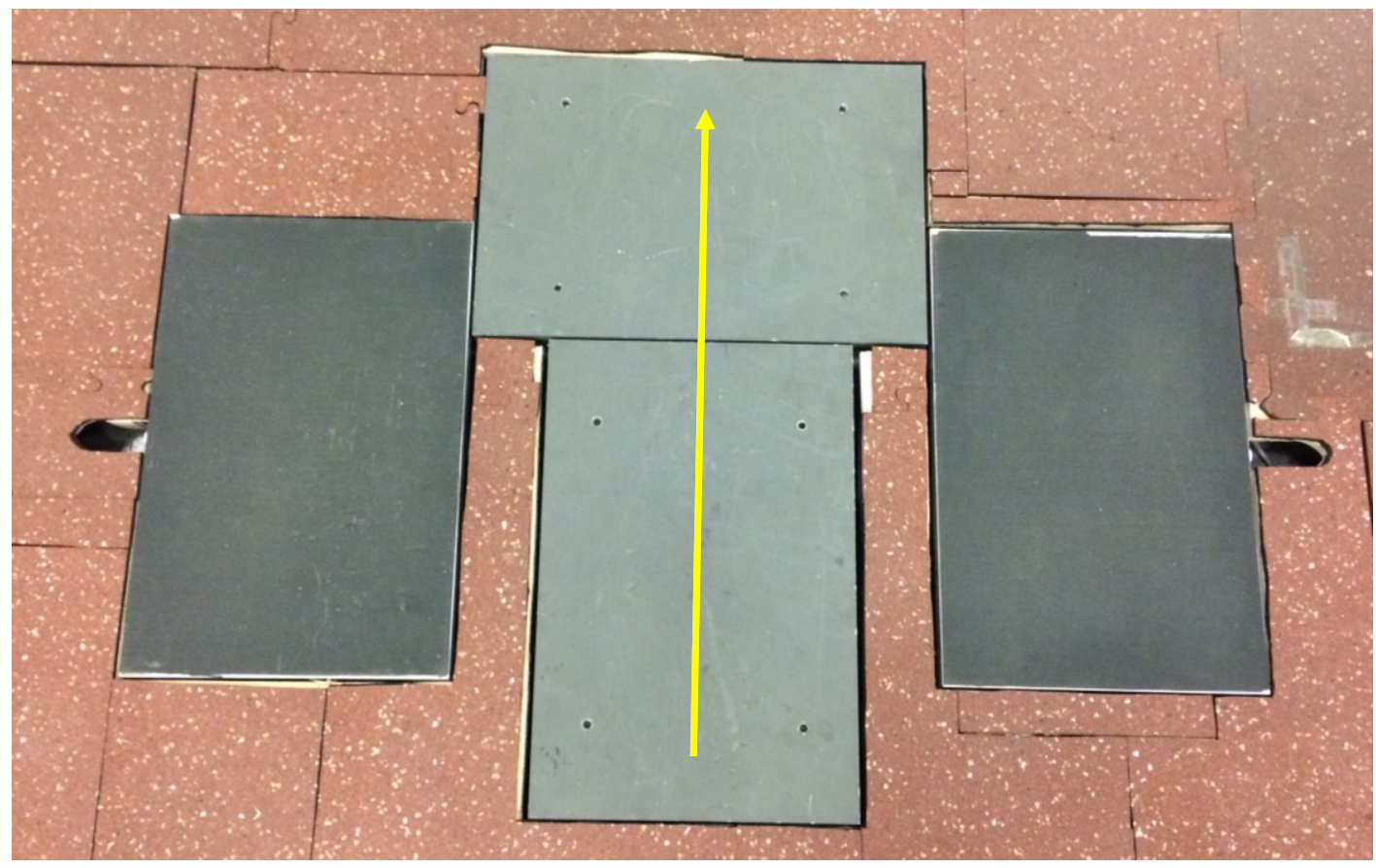

Figure 4.8 Aerial view of the force plate configuration used in Study 2, yellow arrow direction of travel. 


\subsubsection{SEMG - BTS Wireless System}

BTS Bioengineering FreeEMG 300 wireless bipolar electrodes (Figure 4.9) were used for musculoskeletal data collection during this study [119]. Due to the high-mobility of the tasks required, wireless electrodes were determined as the most appropriate style of electrode. Minor issues arose with the wireless system as the battery lifespan of some electrodes had diminished. If an electrode failed and no replacement electrodes were available, the biceps barchii electrodes were used to replace the posteriorly located muscle(s). Two participant testing sessions occurred issue free; see Table 4.4 for electrode replacements. Sweat was a limitation concerning $S E M G$ analysis; pro-wrap and/or surgical tape was used to secure the electrode if warranted. Excessive perspiration was not expected from participants as rest duration was provided between trials, and trials duration did not exceed $7 \mathrm{~s}$. All $s E M G \mathrm{~s}$ data were acquired at $1000 \mathrm{~Hz}$. BTS EMG-Analyzer (version 1.1.2.0, BTS Bioengineering, Brooklyn NY, USA) was the corresponding computer software [119]. $s E M G$ data were not analyzed in this thesis.

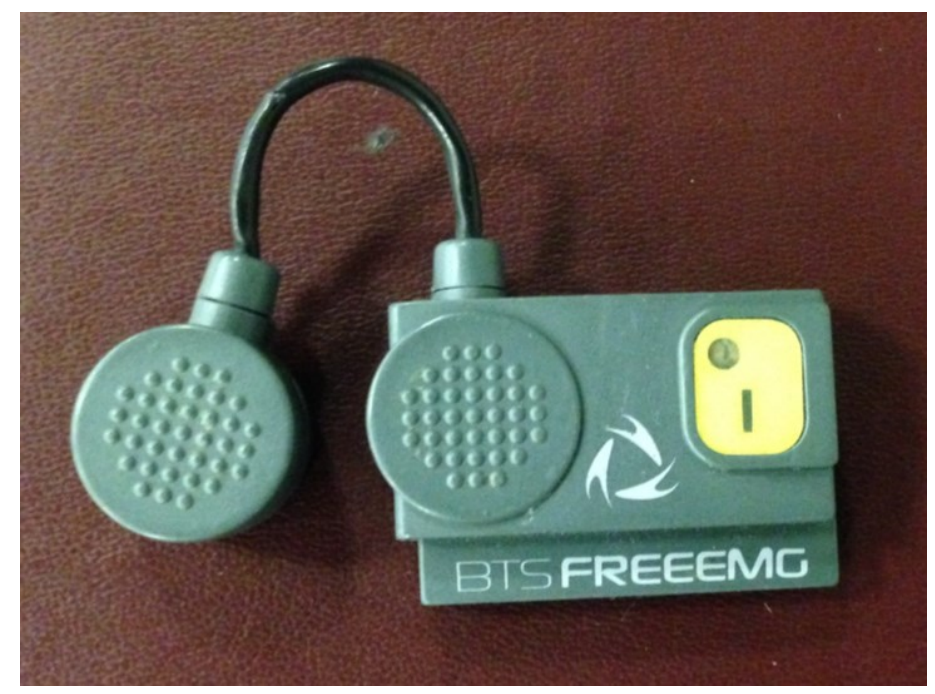

Figure 4.9 BTS bipolar wireless electrode used in Study 2. 
Table 4.4 sEMG electrode replacements for participants in Study 2

\begin{tabular}{lll}
\hline $\mathbf{n = 3}$ & Left Electrode(s) Replaced & Right Electrode(s) Replaced \\
\hline \hline C2 & Trapezius (new) & Triceps Brachii (new) \\
& & Latissimus Dorsi (left Bicep Brachii) \\
C3 & Latissimus Dorsi (left Bicep Brachii) & Posterior Deltoid (right Biceps \\
& & Brachii) \\
C5 & & Latissimus Dorsi (right Bicep \\
& & Brachii) \\
\hline
\end{tabular}

\subsubsection{Data Acquisition, Processing, and Publication}

Study 2 data acquisition, processing, and publication methodologies for MOCAP and force plate data replicated the methodologies outline in Study 1, see Section 3.3.3. Initial contact and push-off locations were marked as events $\left(t_{\text {Impact }} \Rightarrow F_{x} \neq 0 \mathrm{~N}\left\|F_{y} \neq 0 \mathrm{~N}\right\| F_{z} \neq\right.$

$0 \mathrm{~N}$ and $t_{\text {Recoil }} \Rightarrow F_{x}=F_{y}=F_{z}=0 \mathrm{~N}$, respectively). From these locations contact and swing phases were determined, propulsion and recovery, respectively. Preparation analysis began at the downward cycle initial event, and incrementally decreased $\left(t_{i}<t_{j}\right)$ until the datum immediately prior to the initiation of propulsion $(y=0.000 \mathrm{~m})$.

$s E M G$ data were integrated into Vicon Nexus post acquisition. Upon integration, force plate data were automatically converted into a sampling frequency of $1000 \mathrm{~Hz}$.

\subsection{Methodology: Testing Protocol}

This section outlines the methodologies used for Study 2 concerning testing protocols.

\subsubsection{Defining Skating in Sledge Hockey}

Skating in sledge hockey can take many forms; this thesis defines and focuses on the following: 
1. Stroke: measured at pick $y$ trajectory maximum to the immediate return to pick $y$ trajectory maximum of the ipsilateral limb; analogous to a step, stride, and single walking gait cycle [35]. If $M O C A P$ is not available then pick contact to pick contact is suggested with the understanding that the preparation phase of the succeeding cycle belongs to the observed cycle.

where,

$$
\text { stroke rate }=\frac{1}{t_{n+1}-t_{n}}
$$

$$
n=\text { stroke number, } t=\text { time }(\mathrm{s}), t_{n}=\text { start of the } n^{\text {th }} \text { stroke, and } t_{n+1}=
$$

start of the immediate next stroke.

2. Bilateral Skating: understood as double support in gait analysis with symmetrical measures as the desired optimum [35]; that is, the stroke rate of the left arm is equal to the stroke rate of the right arm.

3. Forward Skating: measured as anterior-posterior directional linear propulsion with defined phases: 1) propulsion, and 2) recovery, as outlined in Section 2.1.1. The definition of 3) preparation is re-examined and amended within this thesis.

a. Propulsion: pick contact to pick-off.

b. Recovery: instant immediately post pick-off to instant immediately preceding preparation.

c. Preparation: pick $y$ trajectory maximum during shoulder flexion to the instant immediately preceding to pick contact.

4. Start Stroke: measured from a static start with elevated arms, downward drop initiation to the immediate return to pick trajectory maximum $y$. An additional form of measurement includes duration terminating at the immediate sequential contact 
(initial cycle plus preparation).

5. Mid Stroke: any stroke post start stroke, which may include final stroke.

\subsubsection{Testing Protocol}

Minimum trial numbers were the required total trial numbers for Study 2 participants. No participant performed a perfect testing session (missed trials occurred). Three useable trials were required for each test ( $n=3$ per test). Each participant performed a minimum of 6 submaximal (Test 1 and Test 3 ) and 6 maximal (Test 4 and Test 8 ) unrestricted trials in a semi-randomized order. Two participants $(C 2$ and $C 5)$ performed a minimum of 3 additional maximal (Test 2) unrestricted trials. Random assignment of the additional maximal trials occurred by a coin toss prior to participant preparation. Participants were unaware of the addition or exclusion of the trials. Each participant performed a minimum of 9 maximal (Tests 5-7) restricted trials in a semi-randomized order. In summary, three participants $(C 1, C 3$, and $C 4)$ performed a minimum of $\mathrm{n}=39$ trials, and two participants ( $C 2$ and $C 5$ ) performed a minimum of $\mathrm{n}=42$ trials. The additional 3 trials are indicated by * in all below presented Study 2 data, increasing the participant's Mid $_{\text {Max }}$ condition total trial number to $n=6$. Further details on these tasks are given in subsequent subsections.

Up to a 2-minute rest was allotted between trials with the participant being allowed to stand up in between restricted-elbow tests. Up to five practice trials were allotted prior to Test 1 allowing the participant to become acquainted with the off-ice sledge hockey equipment. Leg-rest length for the sledge was participant specific promoting comfort. 
All trials began with the experimenter providing the verbal cue 'go', followed by the participant-initiated start. All trials were video-recorded providing reviewable data in order to implement the previously outlined observable gait parameters (Section 4.1). Failed trials were repeated until three useable trials for each test were obtained. A failed trial included but was not limited to the participant falling over during the desired observable task (post task imbalance was not seen as a failure), the pick slipping off the force plate during pushoff, or the pick missing the force plate. The testing environment was kept positive and encouraging to promote maximal and focused participation. Physical testing did not exceed 2 hours, well within the 3 hour allotted time, with the majority of participants completing prior to 1.5 hours.

Task order was identical between participants with the exception of restricted elbow angle (see Section 4.4.5). Test order was as follows:

1) Full Free Test - submaximal

2) Full Free Test - maximal $* C 2$ and $C 5$ only

3) Static Free Test - submaximal

4) Static Free Test - maximal

5) Restricted Elbow 1 - Flexed or Extended
a. Above the horizon start height
b. At the horizon start height
c. Below the horizon start height

6) Restricted Elbow 2 - Mid

a. Above the horizon start height 
b. At the horizon start height

c. Below the horizon start height

7) Restricted Elbow 3 - Extended or Flexed

a. Above the horizon start height

b. At the horizon start height

c. Below the horizon start height

8) Full Free Test - maximal

\subsubsection{Full Free Test}

Participant-sledge start location was located off the force plates, one-stroke distance from left/right stick-force plate interface (Figure 4.10). Some participants required an additional shortened stroke prior to the picks contacting the force plates. The addition or exclusion of this shortened stroke was sporadic and participant dependent. Picks were required to be elevated (participant specific) in order to begin the trial. Participant-initiated start stroke occurred propelling the sledge onto the force plates. Data for mid stroke analysis was acquired as the participant travel on, across, and off the force plates. A third/final stroke was allotted if the participant desired; however, a coasting-stop with pick contact for balance typically occurred post push-off from the mid stroke cycle. Figure 4.10, shows a participant at the start position for a Full Free trial. 


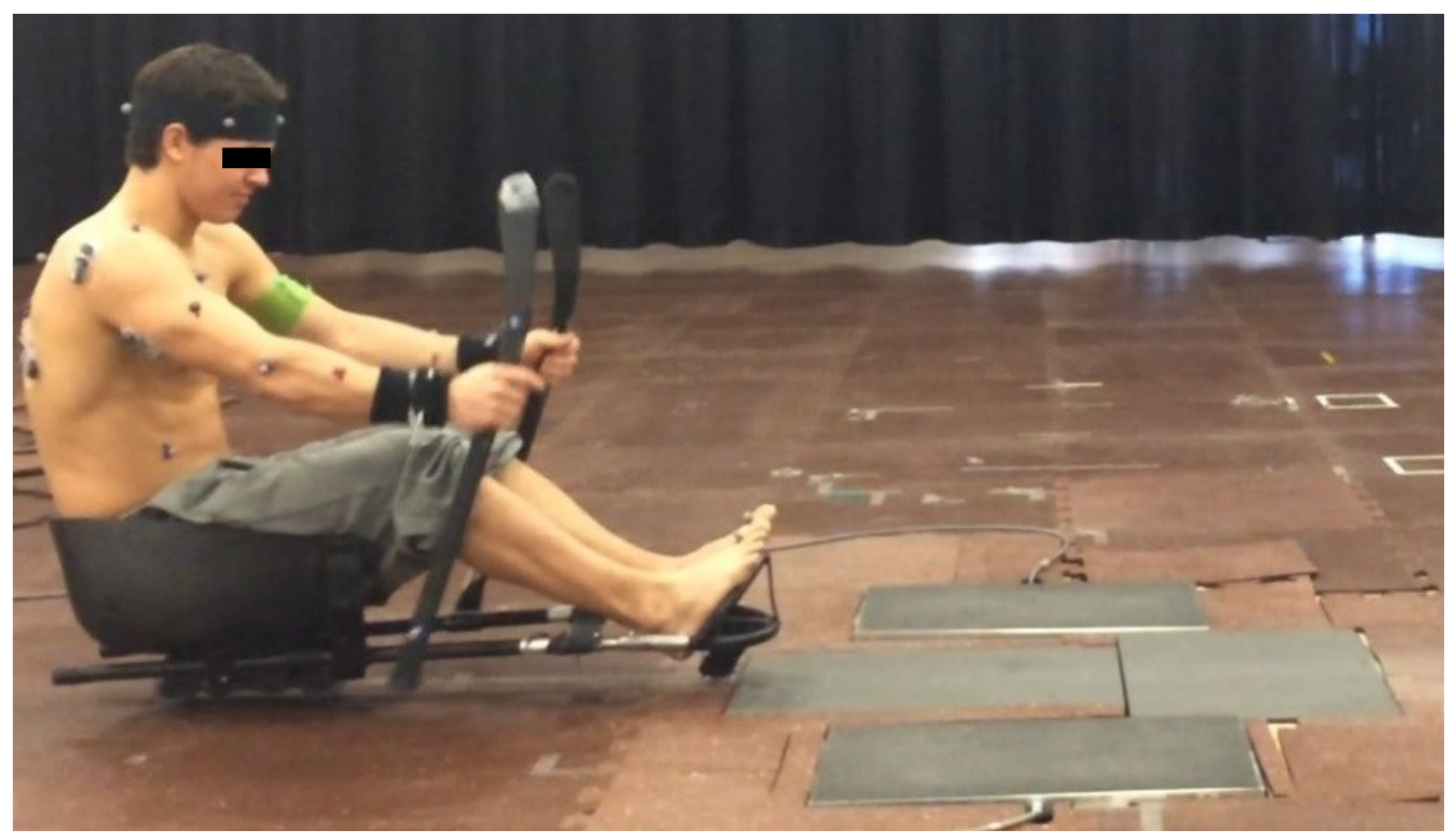

Figure 4.10 Full Free trial start position enabling mid stroke data to be collected upon force plate contact for Study 2.

\subsubsection{Static Free Test}

Participant static start was located on the force plates (Figure 4.11). Picks were required to be elevated (participant specific) in order to begin the trial. Participant-initiated start stroke occurred propelling the sledge off the force plates. A second/final stroke was allotted if the participant desired; however, a coasting-stop with pick contact for balance typically occurred post push-off from the start stroke cycle. Figure 4.11, shows a participant at the start position for Static Free trial. 


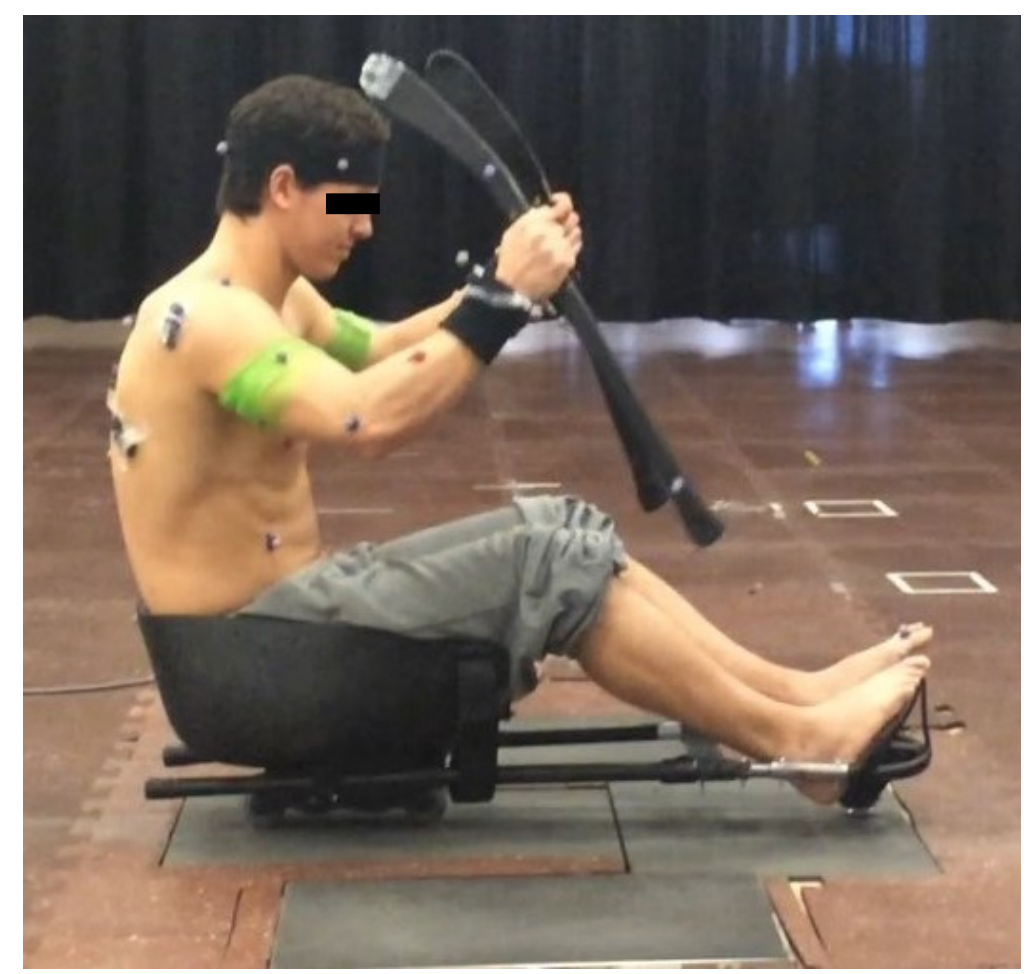

Figure 4.11 Static Free trial start position enabling start stroke data to be collected upon force plate contact for Study 2.

\subsubsection{Restricted Elbow \& Wrist Joints}

Restricted trials mimicked SLAM - 80 methodology outlined in Section 3.3.2. Elbow braces were used to link the participants; final elbow angle of the preceding participant was the initial elbow angle of the current participant. A mid elbow angle was always the middle investigated elbow angle. Due to result similarity observed from investigating drop heights for $S L A M-80$, and the inability for an individual to determine exact upper arm angle, wrist height was used as the determining parameter for drop height start position. This joint was determined as the optimal start parameter based on its ease of gauging for the participant, a transferrable reference point for on-ice game-specific observations for the individual and potential investigator(s). Figure 4.12, shows a participant at the horizontal start position for a restricted trial. 


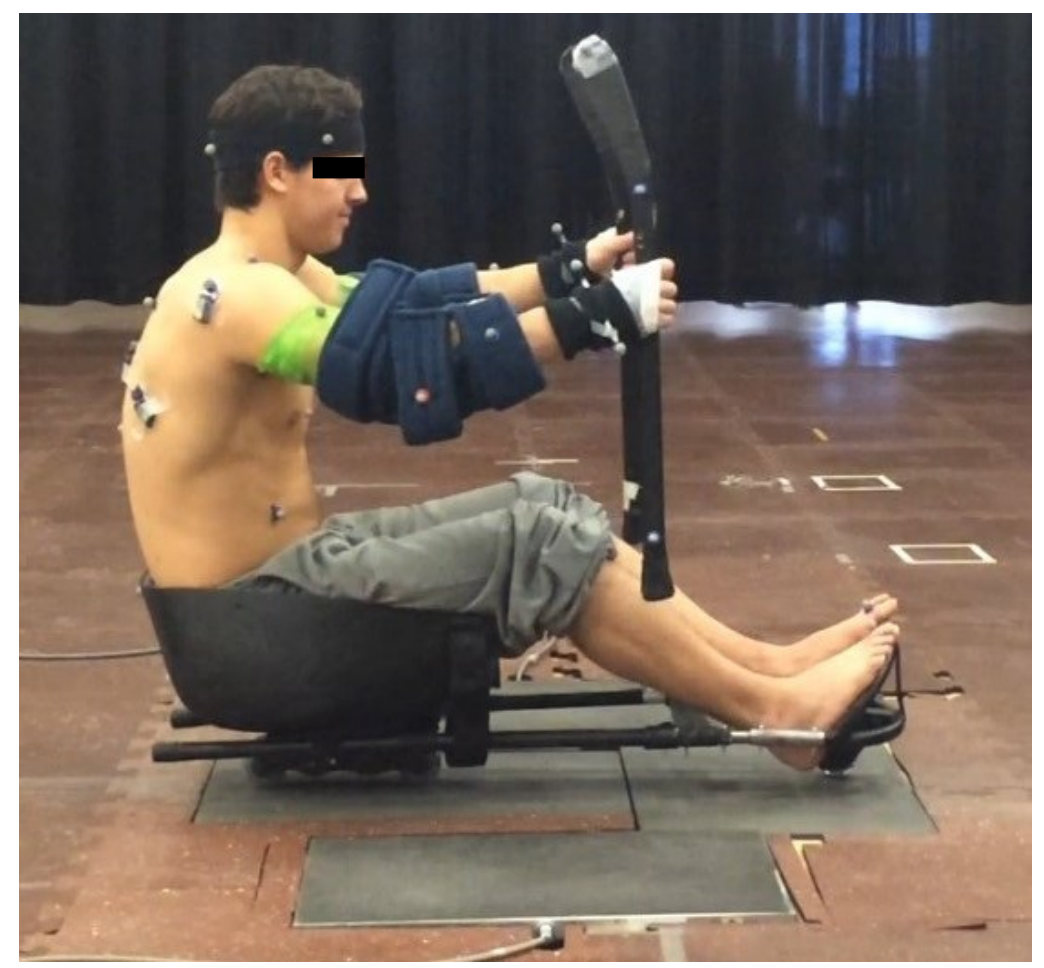

Figure 4.12 Restricted trial horizontal start position enabling impact data to be collected upon force plate contact for Study 2 (similar to Study 1).

\subsubsection{Previous Disseminations of Study 2 Results}

Results from Study 2 discussing stroke kinematics during the preparation and propulsion phases were presented at the Own the Podium Sports Innovation Summit (SPIN 2016), held in Calgary, Canada, in an unpublished abstract, "Differences between sagittal plane static start and mid-cycle stroke biomechanics during skating for the sport of sledge hockey: Task naïve population", off-ice data outlining key areas concerning stick angles during the contact phase, and torso angle during a start and mid stroke in sledge was presented as a poster by the author [120].

Results from Study 2 discussing impact forces and the potential for injury as a result of the propulsion phase were presented at the Own the Podium Sports Innovation Summit (SPIN 
2016 ), held in Calgary, Canada, in an unpublished abstract, "Investigation of the relationship between peak impact and push-off reaction forces, and potential upper limb overuse/overloading injuries introduced from skating in the sport of sledge hockey", office data outlining key areas within the contact phase, which could introduce injury during skating in sledge hockey was presented as a poster by the author [121].

\subsection{Results}

Results are presented in a step-wise analytical approach discussing first the kinematics then the respective derived kinetics for Study 2 acquired data. The same inverse dynamic mathematical model as Study 1 was used to compute kinetic values of each trial at its respective peak impact force $\left(t_{\text {Peak }}\right)$. Results are presented with focus on the start stroke (Start, Static Free tests), and mid stroke (Mid, Full Free tests). Results can then be further presented with focus on submaximal (Sub) and maximal (Max) efforts. Results can also be separated into left $(\mathrm{L})$ and right $(\mathrm{R})$. Results represent the tested population as a whole. Individual participant comparison is provided when relevant; ranking is based upon the total tested population $(n=5)$.

\subsubsection{Sagittal Plane Pick Contact Angles}

Anterior-posterior and longitudinal trajectory data were processed from the downward pole to point of recoil for each test in Study 2. From trajectory data, pick impact angles were computed for the left and right stick for the 1) start stroke, and 2) mid stroke, at a) peak impact force (pick impact angle), and b) pick recoil (pick push-off angle) (Table 4.5). 
Table 4.5 Pick impact and push-off angles for naïve of-ice skating in sledge hockey

\begin{tabular}{|c|c|c|c|c|c|c|c|c|}
\hline \multirow{3}{*}{$\begin{aligned} n & =3 \\
* n & =6\end{aligned}$} & \multicolumn{4}{|c|}{ Pick Impact Angle } & \multicolumn{4}{|c|}{ Pick Push-Off Angle } \\
\hline & \multicolumn{2}{|c|}{ Start Stroke } & \multicolumn{2}{|c|}{ Mid Stroke } & \multicolumn{2}{|c|}{ Start Stroke } & \multicolumn{2}{|c|}{ Mid Stroke } \\
\hline & $\mathbf{L}$ & $\mathbf{R}$ & $\mathbf{L}$ & $\mathbf{R}$ & $\mathbf{L}$ & $\mathbf{R}$ & $\mathbf{L}$ & $\mathbf{R}$ \\
\hline$C 1\left(^{\circ}\right)$ & $\begin{array}{l}87 \pm \\
1\end{array}$ & $\begin{array}{l}85 \pm \\
2\end{array}$ & $\begin{array}{l}81 \pm \\
2\end{array}$ & $\begin{array}{l}85 \pm \\
2\end{array}$ & $\begin{array}{l}88 \pm \\
8\end{array}$ & $\begin{array}{l}88 \pm \\
10\end{array}$ & $\begin{array}{l}92 \pm \\
16\end{array}$ & $\begin{array}{l}94 \pm \\
12\end{array}$ \\
\hline$C 2\left(^{\circ}\right)$ & $\begin{array}{l}83 \pm \\
4\end{array}$ & $\begin{array}{l}84 \pm \\
2\end{array}$ & $\begin{array}{l}85 \pm \\
7 *\end{array}$ & $\begin{array}{l}86 \pm \\
6 *\end{array}$ & $\begin{array}{l}100 \pm \\
56\end{array}$ & $\begin{array}{l}100 \pm \\
60\end{array}$ & $\begin{array}{l}89 \pm \\
32 *\end{array}$ & $\begin{array}{l}55 \pm \\
36 *\end{array}$ \\
\hline$C 3\left(^{\circ}\right)$ & $\begin{array}{l}86 \pm \\
2\end{array}$ & $\begin{array}{l}83 \pm \\
4\end{array}$ & $\begin{array}{l}88 \pm \\
3\end{array}$ & $\begin{array}{l}84 \pm \\
6\end{array}$ & $\begin{array}{l}153 \pm \\
20\end{array}$ & $\begin{array}{l}160 \pm \\
21\end{array}$ & $\begin{array}{l}85 \pm \\
27\end{array}$ & $\begin{array}{l}93 \pm \\
36\end{array}$ \\
\hline$C 4\left(^{\circ}\right)$ & $\begin{array}{l}84 \pm \\
5\end{array}$ & $\begin{array}{l}88 \pm \\
4\end{array}$ & $\begin{array}{l}83 \pm \\
5\end{array}$ & $\begin{array}{l}87 \pm \\
2\end{array}$ & $\begin{array}{l}85 \pm \\
13\end{array}$ & $\begin{array}{l}89 \pm \\
10\end{array}$ & $\begin{array}{l}61 \pm \\
25\end{array}$ & $\begin{array}{l}69 \pm \\
23\end{array}$ \\
\hline$C 5\left(^{\circ}\right)$ & $\begin{array}{l}83 \pm \\
1\end{array}$ & $\begin{array}{l}86 \pm \\
3\end{array}$ & $\begin{array}{l}83 \pm \\
3 *\end{array}$ & $\begin{array}{l}87 \pm \\
1 *\end{array}$ & $\begin{array}{l}84 \pm \\
10\end{array}$ & $\begin{array}{l}82 \pm \\
12\end{array}$ & $\begin{array}{l}68 \pm \\
15 *\end{array}$ & $\begin{array}{l}67 \pm \\
15 *\end{array}$ \\
\hline $\begin{array}{l}\text { Mean } \pm \\
S D\left(^{\circ}\right)\end{array}$ & $\begin{array}{l}85 \pm \\
3\end{array}$ & $\begin{array}{l}85 \pm \\
3\end{array}$ & $\begin{array}{l}84 \pm \\
5\end{array}$ & $\begin{array}{l}86 \pm \\
4\end{array}$ & $\begin{array}{l}102 \pm \\
37\end{array}$ & $\begin{array}{l}104 \pm \\
40\end{array}$ & $\begin{array}{l}79 \pm \\
26\end{array}$ & $\begin{array}{l}82 \pm \\
28\end{array}$ \\
\hline
\end{tabular}

No difference occurred between the stroke styles (start and mid stroke) or limbs (left and right) at peak pick impact. Two distinct stick push-off positions occurred during the start stroke: 1) propulsive $\left(<90^{\circ}\right)$, and 2) breaking $\left(>90^{\circ}\right)$. Start stroke pick push-off angles for $C 3$ were in the least propulsive position $\left(\sim 150^{\circ}\right)$ compared to the other 4 participants $\left(\sim 80^{\circ}-90^{\circ}\right)$. Two similar distinct differences were observed in stick push-off positions during the mid stroke. Most stick angles were in the propulsive position during mid stroke, with a clear distinction between an upright propulsive position, and a performance enhancing position (more horizontal). Mid stroke pick push-off angles for $C 1$ and $C 3$ both sticks, and $C 2$ left stick were in a less propulsive position than $C 4$ and $C 5$, and $C 2$ right stick.

In total three stroking patterns were observed during the propulsion phase in Study 2. The most common motion consisted of the pick contact angle becoming larger (less horizontal) prior to push-off. Figure 4.13 (b) presents a depiction of the stick travelling forward, and 
then returning past peak impact (solid black). This reversal in direction can be interpreted as a pulling motion. As the body moves forward past the point of rotation (shoulder), the elbow flexes instead of extending the forearm creating potential for increased wrist movement, resulting in larger stick angles. Currently, it is unknown if wrist movement influences skating in sledge hockey. Every participant demonstrated this pulling motion at least once for the start stroke, and at least once for the mid stroke tests. The second propulsion phase stroking pattern observed was the predicted pushing motion (acute pick push-off angle, Figure 4.13 (a)). As the body moves forward past the point of rotation (shoulder), the elbow extends the forearm creating potential for increased wrist movement, resulting in smaller stick angles. The final stroking pattern observed was a static held stick indicating the stick was used to maintain body-sledge balance.

Nearly all restricted elbow trials presented similar pulling motion trajectories. Pick pushoff angle was not the focus as restricted trials were designed to mimic $S L A M-80$ (initial drop only). Further discussion regarding the restricted trials occurs in Chapter 5. 

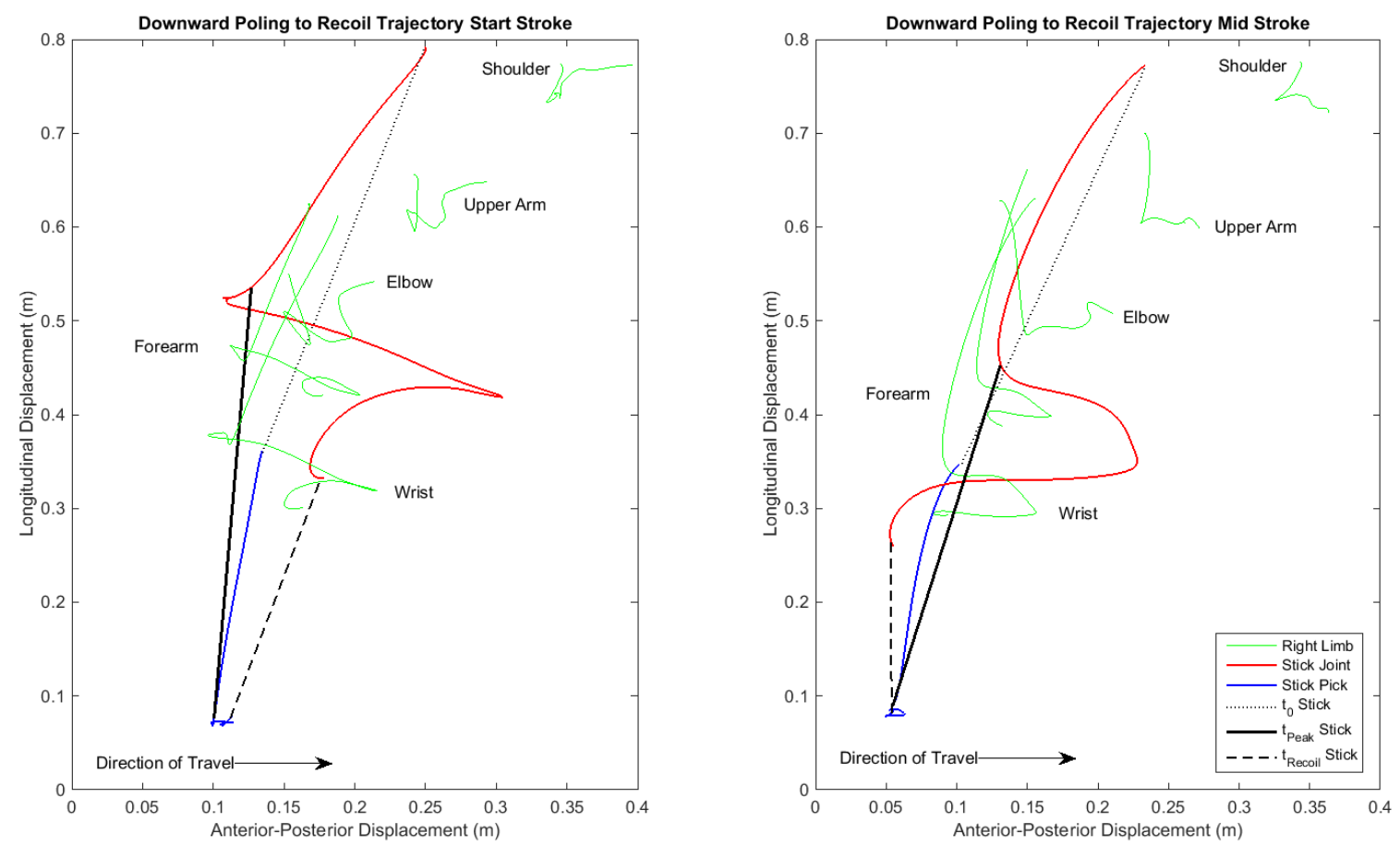

Figure 4.13 Representation for two of the three propulsion phase stroking patterns observed during Study 2. Start stroke (left) and mid stroke (right), right limb (green) and stick (black) trajectories from $t_{0}$ (dotted) to $t_{\text {Recoil }}$ (dash), and at $t_{\text {Peak }}$ (solid). Motion of the stick is provided by the joint of the shaft (red) and pick of stick (blue). Every participant performed at least one pulling motion (right) during the propulsion phase, with one participant pulling every start stroke (C3). The final stroking pattern was a static held stick.

\subsubsection{Impact Force}

Data from peak impact forces establish the foundation for kinetic analysis of the downward pole in the sport of sledge hockey (similar to Study 1). Information regarding peak pushoff force (not $t_{\text {Recoil }}$ ) is also investigated. Peak push-off force is defined as the peak force produced in the second half of total contact length $\left(\left[\left(t_{\text {Impact }}+\frac{t_{\text {Recoil }}-t_{\text {Impact }}}{2}\right): t_{\text {Recoil }}\right]\right)$.

Horizontal force remained under $70 \mathrm{~N}$ for every trial, for every participant. Low horizontal forces are suggested to be a result of the upright stick positions. Left mediolateral mean 
peak impact force was $98 \mathrm{~N} \pm 21 \mathrm{~N}$ for all participants, and right mediolateral mean peak impact force was $119 \mathrm{~N} \pm 23 \mathrm{~N}$. Relatively low mediolateral impact forces support the 2dimensional design of $S L A M-80$. From this, vertical impact force can be determined as the primary production force during Study 2. Results presented are investigated as resultant impact forces (impact force) with an understanding that vertical force is a majority of the resultant value. From this vertical positioning, increased potential risk of propulsion phase introduced injury is probable.

From Table 4.6, all submaximal mean impact forces were less than maximal effort mean impact forces for the respective stroke and limb, with the exception of $C 5$ left and right start strokes. For submaximal and maximal efforts, three participants produced more force during the mid stroke than the start stroke $(C 1, C 4, C 5)$. Peak impact force ranged from $1.58 \times B W$ and direction $96^{\circ}$ to $3.12 \times B W$ and direction $94^{\circ}$ for the left limb, and $1.23 \times B W$ and direction $92^{\circ}$ to $2.30 \times B W$ and direction $96^{\circ}$ for the right limb. Both maximal peaks were produced from the same participant $(C 5)$.

From Table 4.6, the average male ( $82 \mathrm{~kg}$ ) naïve sledge hockey player produces approximately $0.9 \times B W$ and direction $96^{\circ}$ of impact force from submaximal efforts for the left start and mid strokes, and approximately $0.8 \times B W$ and direction $98^{\circ}$ of impact force from right submaximal effort start and mid strokes. The average male naïve sledge hockey player produces approximately $1.2 \times B W$ and direction $98^{\circ}$ of force from impact for right limb maximal efforts during the start and mid stroke. Maximal efforts for the left limb start and mid strokes produce approximately $1.4 \times B W$ and direction $96^{\circ}$, and 
$1.7 \times B W$ and direction $96^{\circ}$ of force from impact, respectively.

From Table 4.7, only two participants $(C 1, C 5)$ produced less submaximal mean push-off forces than compared to the maximal effort mean push-off forces for the respective stroke and limb. Overall, there was no difference between submaximal and maximal effort at peak push-off. For submaximal and maximal efforts, two participants ( $C 3$ and $C 5)$ produced more mean push-off force during the start stroke than the mid stroke, for both limbs. Peak push-off force ranged from $0.26 \times B W$ and direction $94^{\circ}$ to $0.39 \times B W$ and direction $91^{\circ}$ for the left limb, and $0.30 \times B W$ to $0.37 \times B W$ for the right limb.

One participant (C4) displayed bilateral symmetry, and was the only identified bilateral participant within this study. This participant (C4) was the only participant with limited experience with sledge hockey, and had expertise in an on-ice sport. Coincidently, C4 was the only participant with identical limb mean peak impact force $(921 \mathrm{~N})$, computed for every trial $(n=39)$.

Figure 4.14 illustrates resultant reaction force curves for all force plates for a Full Free trial. During mid stroke, the left limb (blue) produced a greater peak impact force (dashed) compared to the right limb (red), and the $S L A M-80$ mean impact force baseline (green). Smoother waveforms are observed throughout the left stick impact force curves compared to the minutely-fluctuating right limb signatures, suggesting a more fluent left limb stroke. 
Table 4.6 Left vs right limb propulsion phase resultant impact forces for Study 2

\begin{tabular}{|c|c|c|c|c|c|c|c|c|c|c|}
\hline \multirow{2}{*}{$\begin{array}{l}n=3 \text { per } \\
\text { condition } \\
* n=6\end{array}$} & \multicolumn{3}{|c|}{ Start Stroke } & \multicolumn{4}{|c|}{ Mid Stroke } & \multicolumn{3}{|c|}{ Peak } \\
\hline & \multicolumn{2}{|l|}{$\mathbf{L}$} & \multirow[t]{2}{*}{$\mathbf{R}$} & & \multicolumn{2}{|l|}{$\mathbf{L}$} & \multicolumn{2}{|c|}{$\mathbf{R}$} & \multirow[t]{2}{*}{$\mathbf{L}$} & \multirow[t]{2}{*}{$\mathbf{R}$} \\
\hline \multicolumn{8}{|c|}{ Submaximal } & & & \\
\hline \multirow[t]{3}{*}{$C 1(B W)$} & 0.39 & $97^{\circ}$ & 0.32 & $95^{\circ}$ & 0.83 & $96^{\circ}$ & 0.87 & $96^{\circ}$ & & \\
\hline & \pm & \pm & \pm & \pm & \pm & \pm & \pm & \pm & & \\
\hline & 0.10 & $1^{\circ}$ & 0.06 & $1^{\circ}$ & 0.04 & $2^{\circ}$ & 0.19 & $1^{\circ}$ & & \\
\hline \multirow[t]{3}{*}{$C 2(B W)$} & 0.83 & $97^{\circ}$ & 0.66 & $103^{\circ}$ & 0.57 & $97^{\circ}$ & 0.55 & $106^{\circ}$ & & \\
\hline & \pm & \pm & \pm & \pm & \pm & \pm & \pm & \pm & & \\
\hline & 0.13 & $1^{\circ}$ & 0.14 & $2^{\circ}$ & 0.37 & $1^{\circ}$ & 0.11 & $5^{\circ}$ & & \\
\hline \multirow[t]{3}{*}{$C 3(B W)$} & 0.92 & $95^{\circ}$ & 0.64 & $97^{\circ}$ & 0.73 & $99^{\circ}$ & 0.62 & $99^{\circ}$ & & \\
\hline & \pm & \pm & \pm & \pm & \pm & \pm & \pm & \pm & & \\
\hline & 0.18 & $0^{\circ}$ & 0.18 & $2^{\circ}$ & 0.26 & $7^{\circ}$ & 0.01 & $1^{\circ}$ & & \\
\hline \multirow[t]{3}{*}{$C 4(B W)$} & 1.05 & $96^{\circ}$ & 0.85 & $96^{\circ}$ & 1.29 & $95^{\circ}$ & 0.89 & $94^{\circ}$ & & \\
\hline & \pm & \pm & \pm & \pm & \pm & \pm & \pm & \pm & & \\
\hline & 0.31 & $1^{\circ}$ & 0.16 & $1^{\circ}$ & 0.15 & $1^{\circ}$ & 0.39 & $2^{\circ}$ & & \\
\hline \multirow[t]{3}{*}{$C 5(B W)$} & 1.40 & $98^{\circ}$ & 1.49 & $97^{\circ}$ & 1.89 & $96^{\circ}$ & 1.76 & $96^{\circ}$ & & \\
\hline & \pm & \pm & \pm & \pm & \pm & \pm & \pm & \pm & & \\
\hline & 0.12 & $0^{\circ}$ & 0.19 & $2^{\circ}$ & 0.50 & $2^{\circ}$ & 0.35 & $1^{\circ}$ & & \\
\hline \multirow{3}{*}{$\begin{array}{l}\text { Mean } \pm \\
S D(B W)\end{array}$} & 0.92 & $96^{\circ}$ & 0.79 & $97^{\circ}$ & 0.91 & $96^{\circ}$ & 0.79 & $98^{\circ}$ & & \\
\hline & \pm & \pm & \pm & \pm & \pm & \pm & \pm & \pm & & \\
\hline & 0.37 & $1^{\circ}$ & 0.40 & $3^{\circ}$ & $\mathbf{0 . 3 3}$ & $3^{\circ}$ & 0.22 & $5^{\circ}$ & & \\
\hline \multicolumn{11}{|c|}{ Maximal } \\
\hline \multirow[t]{3}{*}{$C 1(B W)$} & 0.85 & $95^{\circ}$ & 0.70 & $98^{\circ}$ & 1.62 & $97^{\circ}$ & 1.08 & $95^{\circ}$ & 2.00 & 1.23 \\
\hline & \pm & \pm & \pm & \pm & \pm & \pm & \pm & \pm & & \\
\hline & 0.09 & $1^{\circ}$ & 0.16 & $1^{\circ}$ & 0.33 & $2^{\circ}$ & 0.16 & $2^{\circ}$ & $95^{\circ}$ & $92^{\circ}$ \\
\hline \multirow[t]{4}{*}{$C 2(B W)$} & 1.26 & $98^{\circ}$ & 1.08 & $100^{\circ}$ & 0.98 & $97^{\circ}$ & 0.70 & $100^{\circ}$ & 1.58 & 1.42 \\
\hline & \pm & \pm & \pm & \pm & \pm & \pm & \pm & \pm & & \\
\hline & 0.11 & $1^{\circ}$ & 0.07 & $1^{\circ}$ & 0.18 & $1^{\circ}$ & 0.15 & $6^{\circ}$ & $96^{\circ}$ & $100^{\circ}$ \\
\hline & & & & & $*$ & $*$ & $*$ & $*$ & & \\
\hline \multirow[t]{3}{*}{$C 3(B W)$} & 1.87 & $93^{\circ}$ & 1.38 & $96^{\circ}$ & 1.87 & $95^{\circ}$ & 1.27 & $96^{\circ}$ & 2.45 & 2.10 \\
\hline & \pm & \pm & \pm & \pm & \pm & \pm & \pm & \pm & & \\
\hline & 0.45 & $2^{\circ}$ & 0.11 & $1^{\circ}$ & 0.52 & $1^{\circ}$ & 0.26 & $3^{\circ}$ & $94^{\circ}$ & $96^{\circ}$ \\
\hline \multirow[t]{3}{*}{$C 4(B W)$} & 1.23 & $94^{\circ}$ & 1.15 & $95^{\circ}$ & 1.34 & $95^{\circ}$ & 2.06 & $94^{\circ}$ & 1.77 & 1.96 \\
\hline & \pm & \pm & \pm & \pm & \pm & \pm & \pm & \pm & & \\
\hline & 0.10 & $1^{\circ}$ & 0.03 & $1^{\circ}$ & 0.36 & $3^{\circ}$ & 0.35 & $1^{\circ}$ & $92^{\circ}$ & $93^{\circ}$ \\
\hline \multirow[t]{4}{*}{$C 5(B W)$} & 1.22 & $96^{\circ}$ & 1.10 & $98^{\circ}$ & 2.47 & $96^{\circ}$ & 1.84 & $96^{\circ}$ & 3.12 & 2.30 \\
\hline & & \pm & \pm & \pm & \pm & \pm & \pm & \pm & & \\
\hline & 0.36 & $1^{\circ}$ & 0.10 & $2^{\circ}$ & 0.47 & $3^{\circ}$ & 0.31 & $2^{\circ}$ & $94^{\circ}$ & $98^{\circ}$ \\
\hline & & & & & $*$ & $*$ & $*$ & $*$ & & \\
\hline$\overline{~ M e a n ~} \pm$ & $\overline{1.44}$ & $96^{\circ}$ & 1.23 & $98^{\circ}$ & 1.68 & $96^{\circ}$ & 1.29 & $97^{\circ}$ & & \\
\hline$S D(B W)$ & \pm & \pm & \pm & \pm & \pm & \pm & \pm & \pm & & \\
\hline & 0.55 & $2^{\circ}$ & 0.42 & $3^{\circ}$ & 0.66 & $2^{\circ}$ & 0.48 & $4^{\circ}$ & & \\
\hline
\end{tabular}


Table 4.7 Left vs right limb propulsion phase push-off forces for Study 2

\begin{tabular}{|c|c|c|c|c|c|c|c|c|c|c|}
\hline \multirow{2}{*}{$\begin{array}{l}n=3 \text { per } \\
\text { condition } \\
* n=6\end{array}$} & \multicolumn{3}{|c|}{ Start Stroke } & \multicolumn{4}{|c|}{ Mid Stroke } & \multicolumn{3}{|c|}{ Peak } \\
\hline & \multicolumn{2}{|l|}{$\mathbf{L}$} & \multirow[t]{2}{*}{$\mathbf{R}$} & \multicolumn{3}{|c|}{$\mathbf{L}$} & \multicolumn{2}{|l|}{$\mathbf{R}$} & \multirow[t]{2}{*}{$\mathbf{L}$} & \multirow[t]{2}{*}{$\mathbf{R}$} \\
\hline & & & & & bmaxi & & & & & \\
\hline \multirow[t]{3}{*}{$C 1(B W)$} & 0.19 & $93^{\circ}$ & 0.22 & $93^{\circ}$ & 0.26 & $95^{\circ}$ & 0.30 & $91^{\circ}$ & & \\
\hline & \pm & \pm & \pm & \pm & \pm & \pm & \pm & \pm & & \\
\hline & 0.02 & $1^{\circ}$ & 0.03 & $2^{\circ}$ & 0.04 & $4^{\circ}$ & 0.04 & $0^{\circ}$ & & \\
\hline \multirow[t]{3}{*}{$C 2(B W)$} & 0.23 & $99^{\circ}$ & 0.30 & $100^{\circ}$ & 0.22 & $97^{\circ}$ & 0.32 & $97^{\circ}$ & 0.28 & 0.36 \\
\hline & \pm & \pm & \pm & \pm & \pm & \pm & \pm & \pm & & \\
\hline & 0.04 & $4^{\circ}$ & 0.06 & $1^{\circ}$ & 0.00 & $5^{\circ}$ & 0.04 & $2^{\circ}$ & $95^{\circ}$ & $96^{\circ}$ \\
\hline \multirow[t]{3}{*}{$C 3(B W)$} & 0.26 & $95^{\circ}$ & 0.26 & $94^{\circ}$ & 0.18 & $95^{\circ}$ & 0.20 & $96^{\circ}$ & 0.39 & 0.30 \\
\hline & \pm & \pm & \pm & \pm & \pm & \pm & \pm & \pm & & \\
\hline & 0.02 & $4^{\circ}$ & 0.04 & $5^{\circ}$ & 0.02 & $3^{\circ}$ & 0.01 & $1^{\circ}$ & $91^{\circ}$ & $99^{\circ}$ \\
\hline \multirow[t]{3}{*}{$C 4(B W)$} & 0.22 & $91^{\circ}$ & 0.24 & $92^{\circ}$ & 0.22 & $100^{\circ}$ & 0.26 & $92^{\circ}$ & & \\
\hline & \pm & \pm & \pm & \pm & \pm & \pm & \pm & \pm & & \\
\hline & 0.02 & $1^{\circ}$ & 0.02 & $3^{\circ}$ & 0.02 & $10^{\circ}$ & 0.01 & $4^{\circ}$ & & \\
\hline \multirow[t]{3}{*}{$C 5(B W)$} & 0.25 & $102^{\circ}$ & 0.28 & $106^{\circ}$ & 0.19 & $99^{\circ}$ & 0.23 & $94^{\circ}$ & 0.30 & \\
\hline & \pm & \pm & \pm & \pm & \pm & \pm & \pm & \pm & & \\
\hline & 0.04 & $3^{\circ}$ & 0.04 & $3^{\circ}$ & 0.03 & $5^{\circ}$ & 0.03 & $3^{\circ}$ & $106^{\circ}$ & \\
\hline & & & & & Maxim & & & & & \\
\hline \multirow[t]{3}{*}{$C 1(B W)$} & 0.30 & $92^{\circ}$ & 0.29 & $95^{\circ}$ & 0.31 & $97^{\circ}$ & 0.32 & $94^{\circ}$ & 0.34 & 0.37 \\
\hline & \pm & \pm & \pm & \pm & \pm & \pm & \pm & \pm & & \\
\hline & 0.02 & $0^{\circ}$ & 0.02 & $3^{\circ}$ & 0.03 & $8^{\circ}$ & 0.04 & $1^{\circ}$ & $94^{\circ}$ & $97^{\circ}$ \\
\hline \multirow[t]{4}{*}{$C 2(B W)$} & 0.24 & $99^{\circ}$ & 0.30 & $98^{\circ}$ & 0.25 & $95^{\circ}$ & 0.31 & $104^{\circ}$ & & \\
\hline & \pm & \pm & \pm & \pm & \pm & \pm & \pm & \pm & & \\
\hline & 0.01 & $6^{\circ}$ & 0.03 & $1^{\circ}$ & 0.18 & $6^{\circ}$ & 0.03 & $11^{\circ}$ & & \\
\hline & & & & & $*$ & $*$ & $*$ & $*$ & & \\
\hline \multirow[t]{3}{*}{$C 3(B W)$} & 0.23 & $91^{\circ}$ & 0.24 & $95^{\circ}$ & 0.18 & $94^{\circ}$ & 0.22 & $97^{\circ}$ & & \\
\hline & \pm & \pm & \pm & \pm & \pm & \pm & \pm & \pm & & \\
\hline & 0.00 & $0^{\circ}$ & 0.03 & $1^{\circ}$ & 0.01 & $3^{\circ}$ & 0.03 & $9^{\circ}$ & & \\
\hline \multirow[t]{3}{*}{$C 4(B W)$} & 0.22 & $96^{\circ}$ & 0.22 & $96^{\circ}$ & 0.22 & $95^{\circ}$ & 0.26 & $91^{\circ}$ & 0.26 & 0.30 \\
\hline & \pm & \pm & \pm & \pm & \pm & \pm & \pm & \pm & & \\
\hline & 0.02 & $8^{\circ}$ & 0.02 & $1^{\circ}$ & 0.01 & $5^{\circ}$ & 0.01 & $1^{\circ}$ & $94^{\circ}$ & $91^{\circ}$ \\
\hline \multirow[t]{4}{*}{$C 5(B W)$} & 0.27 & $99^{\circ}$ & 0.31 & $98^{\circ}$ & 0.22 & $102^{\circ}$ & 0.28 & $96^{\circ}$ & & 0.33 \\
\hline & \pm & \pm & \pm & \pm & \pm & \pm & \pm & \pm & & \\
\hline & 0.04 & $10^{\circ}$ & 0.01 & $8^{\circ}$ & 0.02 & $10^{\circ}$ & 0.04 & $6^{\circ}$ & & $103^{\circ}$ \\
\hline & & & & & $*$ & $*$ & $*$ & $*$ & & \\
\hline
\end{tabular}

Body-sledge resultant force curves are represented with data from the first force plate (black), as the body and sledge travel prior to and during stick impact. As the participant 
continues to travel through the stroke, the second force plate (grey) provides body-sledge data until push-off. At peak impact ( $\sim \mathrm{s})$ all participant-sledge-stick points of contact had individual force plate interfaces.

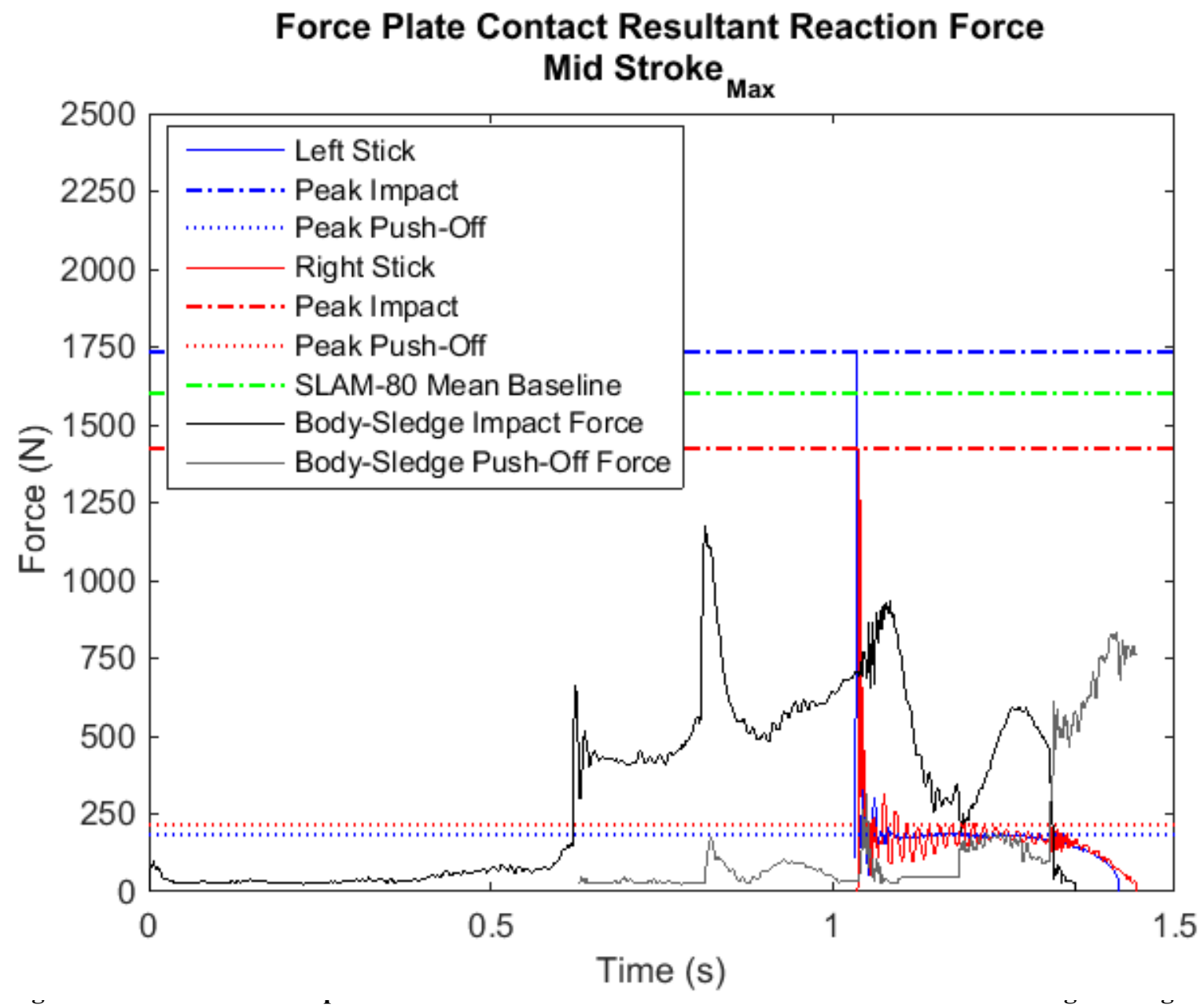

hockey off-ice (left stick - blue, right stick - red, impact body-sledge - black, push-off body-sledge grey). Left stick force curves indicate a more fluent stroke than the right (fluctuation). SLAM - 80 baseline for mean impact force $(1609 \mathrm{~N})$ is indicated in green. 


\subsubsection{Estimated Joint Reaction Force at the Shoulder for a Downward Pole in Sledge Hockey}

From Newton's third law, $J R F_{\text {Shoulder }}$ (force at the shoulder derived from inverse dynamics for human participants) is a result of impact force created from the biomechanical system (upper limb). Comparing Table 4.8 and Table 4.6, JRF $F_{\text {Shoulder }}>$ peak impact force. In general, an increase of approximately $0.2 \times B W$ of force per limb, per stroke is generated as a result of skating impact from naïve sledge hockey players. Similar patterns are observed in Table 4.8 as in Table 4.6 when concerned with stroke style, limb dominance, and effort level. From Table 4.8, submaximal effort for the start and mid strokes generate approximately $1.0 \times B W$ and direction $95^{\circ}$ of force back onto each shoulder for the average male naïve sledge hockey player. Maximal efforts for the right limb start and mid strokes generate approximately $1.5 \times B W$ and direction $86^{\circ}$ of force back onto the shoulder, and maximal efforts for the left limb start and mid strokes generate approximately $1.6 \times B W$ and direction $87^{\circ}$, and $1.9 \times B W$ and direction $86^{\circ}$ of force back onto shoulder, respectively. 
Table 4.8 Estimated $J R F_{\text {Shoulder }}$ for human participants at peak impact force

\begin{tabular}{|c|c|c|c|c|c|c|c|c|c|c|}
\hline \multirow{2}{*}{$\begin{array}{l}\mathrm{n}=3 \text { per } \\
\text { condition } \\
* n=6\end{array}$} & \multicolumn{4}{|c|}{ Start Stroke } & \multicolumn{6}{|c|}{ Mid Stroke } \\
\hline & \multicolumn{2}{|l|}{$\mathbf{L}$} & \multirow[t]{2}{*}{$\mathbf{R}$} & & \multicolumn{2}{|l|}{$\mathbf{L}$} & \multicolumn{2}{|l|}{$\mathbf{R}$} & \multicolumn{2}{|c|}{ Mean } \\
\hline \multicolumn{10}{|c|}{ "Submaximal } & \\
\hline \multirow[t]{3}{*}{$C 1(B W)$} & 0.49 & $87^{\circ}$ & 0.42 & $87^{\circ}$ & 1.00 & $85^{\circ}$ & 1.04 & $86^{\circ}$ & 0.74 & $86^{\circ}$ \\
\hline & \pm & \pm & \pm & \pm & \pm & \pm & \pm & \pm & \pm & \pm \\
\hline & 0.11 & $1^{\circ}$ & 0.07 & $1^{\circ}$ & 0.05 & $1^{\circ}$ & 0.15 & $2^{\circ}$ & 0.31 & $1^{\circ}$ \\
\hline \multirow[t]{3}{*}{$C 2(B W)$} & 0.95 & $82^{\circ}$ & 0.78 & $85^{\circ}$ & 0.68 & $83^{\circ}$ & 0.65 & $83^{\circ}$ & 0.77 & $83^{\circ}$ \\
\hline & \pm & \pm & \pm & \pm & \pm & \pm & \pm & \pm & \pm & \pm \\
\hline & 0.14 & $1^{\circ}$ & 0.16 & $2^{\circ}$ & 0.39 & $4^{\circ}$ & 0.12 & $3^{\circ}$ & 0.23 & $3^{\circ}$ \\
\hline \multirow[t]{3}{*}{$C 3(B W)$} & 1.07 & $86^{\circ}$ & 0.78 & $87^{\circ}$ & 0.87 & $89^{\circ}$ & 0.75 & $84^{\circ}$ & 0.87 & $86^{\circ}$ \\
\hline & \pm & \pm & \pm & \pm & \pm & \pm & \pm & \pm & \pm & \pm \\
\hline & 0.19 & $2^{\circ}$ & 0.12 & $2^{\circ}$ & 0.29 & $1^{\circ}$ & 0.02 & $2^{\circ}$ & 0.20 & $3^{\circ}$ \\
\hline \multirow[t]{3}{*}{$C 4(B W)$} & 1.26 & $83^{\circ}$ & 1.03 & $88^{\circ}$ & 1.50 & $85^{\circ}$ & 1.06 & $86^{\circ}$ & 1.21 & $86^{\circ}$ \\
\hline & \pm & \pm & \pm & \pm & \pm & \pm & \pm & \pm & & \pm \\
\hline & 0.33 & $5^{\circ}$ & 0.17 & $4^{\circ}$ & 0.15 & $5^{\circ}$ & 0.42 & $2^{\circ}$ & 0.32 & $4^{\circ}$ \\
\hline \multirow[t]{3}{*}{$C 5(B W)$} & 1.66 & $83^{\circ}$ & 1.74 & $88^{\circ}$ & 1.40 & $85^{\circ}$ & 1.29 & $87^{\circ}$ & 2.16 & $86^{\circ}$ \\
\hline & & \pm & \pm & \pm & \pm & \pm & \pm & \pm & & \pm \\
\hline & 0.13 & $1^{\circ}$ & 0.20 & $2^{\circ}$ & 0.36 & $3^{\circ}$ & 0.12 & $3^{\circ}$ & 0.27 & $3^{\circ}$ \\
\hline \multirow{3}{*}{$S D(B W)$} & $\begin{array}{l}1.09 \\
\end{array}$ & $184^{\circ}$ & 0.94 & $287^{\circ}$ & 1.07 & $185^{\circ}$ & $\begin{array}{c}0.94 \\
\end{array}$ & $\overline{185^{\circ}}$ & & \\
\hline & & \pm & \pm & \pm & \pm & \pm & \pm & \pm & & \\
\hline & 0.42 & $3^{\circ}$ & 0.45 & $3^{\circ}$ & 0.35 & $3^{\circ}$ & 0.25 & $3^{\circ}$ & & \\
\hline \multicolumn{11}{|l|}{ 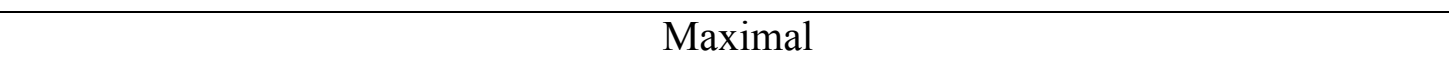 } \\
\hline \multirow[t]{3}{*}{$C 1(B W)$} & 1.00 & $87^{\circ}$ & 0.85 & $86^{\circ}$ & 1.79 & $82^{\circ}$ & 1.25 & $82^{\circ}$ & 1.22 & $84^{\circ}$ \\
\hline & & \pm & \pm & \pm & \pm & \pm & \pm & \pm & & \pm \\
\hline & 0.10 & $1^{\circ}$ & 0.18 & $1^{\circ}$ & 0.34 & $2^{\circ}$ & 0.15 & $3^{\circ}$ & 0.42 & $3^{\circ}$ \\
\hline \multirow[t]{3}{*}{$C 2(B W)$} & 1.42 & $87^{\circ}$ & 1.25 & $84^{\circ}$ & 1.09 & $89^{\circ}$ & 0.83 & $87^{\circ}$ & 1.08 & $87^{\circ}$ \\
\hline & $\begin{array}{l}1.42 \\
\pm\end{array}$ & $\begin{array}{l}8 / \\
\pm\end{array}$ & $\begin{array}{l}1.25 \\
\pm\end{array}$ & $\begin{array}{l}84 \\
\pm\end{array}$ & \pm & \pm & \pm & \pm & & \pm \\
\hline & 0.12 & $\begin{array}{l} \pm \\
2^{\circ}\end{array}$ & 0.10 & $\begin{array}{l} \pm \\
2^{\circ}\end{array}$ & $\begin{array}{l}0.18 \\
*\end{array}$ & $\begin{array}{l}7^{\circ} \\
*\end{array}$ & $\begin{array}{l}0.48 \\
*\end{array}$ & $\begin{array}{l}5^{\circ} \\
*\end{array}$ & 0.28 & $5^{\circ}$ \\
\hline \multirow[t]{3}{*}{$C 3(B W)$} & 2.09 & $87^{\circ}$ & 1.60 & $83^{\circ}$ & 2.13 & $90^{\circ}$ & 1.51 & $86^{\circ}$ & 1.83 & $86^{\circ}$ \\
\hline & \pm & \pm & \pm & \pm & \pm & \pm & \pm & \pm & & \pm \\
\hline & 0.46 & $3^{\circ}$ & 0.14 & $4^{\circ}$ & 0.55 & $3^{\circ}$ & 0.29 & $7^{\circ}$ & 0.44 & $2^{\circ}$ \\
\hline \multirow[t]{3}{*}{$C 4(B W)$} & 1.44 & $88^{\circ}$ & 1.36 & $88^{\circ}$ & 1.53 & $86^{\circ}$ & 1.90 & $88^{\circ}$ & 1.55 & $87^{\circ}$ \\
\hline & \pm & \pm & \pm & \pm & \pm & \pm & \pm & \pm & \pm & \pm \\
\hline & 0.10 & $6^{\circ}$ & 0.03 & $1^{\circ}$ & 0.38 & $5^{\circ}$ & 0.37 & $1^{\circ}$ & 0.32 & $4^{\circ}$ \\
\hline \multirow[t]{4}{*}{$C 5(B W)$} & 2.21 & $84^{\circ}$ & 2.03 & $87^{\circ}$ & 2.79 & $84^{\circ}$ & 2.14 & $87^{\circ}$ & 2.35 & $85^{\circ}$ \\
\hline & \pm & \pm & \pm & \pm & \pm & \pm & \pm & \pm & & \pm \\
\hline & 0.51 & $2^{\circ}$ & 0.40 & $2^{\circ}$ & 0.54 & $3^{\circ}$ & 0.43 & $1^{\circ}$ & 0.54 & $3^{\circ}$ \\
\hline & & & & & $*$ & $*$ & $*$ & $*$ & & \\
\hline \multirow{3}{*}{$\begin{array}{l}\text { Mean } \pm \\
S D(B W)\end{array}$} & 1.61 & $87^{\circ}$ & $\begin{array}{l}1.43 \\
\end{array}$ & $28^{26^{\circ}}$ & 1.89 & $8^{86^{\circ}}$ & $\begin{array}{l}1.51 \\
\end{array}$ & $86^{\circ}$ & & \\
\hline & & \pm & & \pm & \pm & \pm & \pm & \pm & & \\
\hline & 0.60 & $3^{\circ}$ & 0.47 & $3^{\circ}$ & 0.73 & $5^{\circ}$ & 0.55 & $4^{\circ}$ & & \\
\hline
\end{tabular}




\subsubsection{Performance and Potential Injury Indicators for Skating in Sledge Hockey}

As previously stated in Study 1, torque and impulse can provide key information with regards performance enhancement and injury prevention. Again sport-specifically, torque produced from skating in sledge hockey defines the amount of force required through the upper limbs to propel the body and sledge forward. For para-athletes, torque produced about the shoulders can provide vital information, which can potentially identify bodyweight poling as a mechanism of injury. Positive torque is represented by flexion of the shoulder, opposite the downward stroke motion. Torque is dependent upon force, in this case, peak impact force. Torque is also dependent upon the distance from the line of action, in this case, a flexed elbow and/or upright stick position can decrease the moment arm for each segment considerably affecting torque about the shoulder with respect to the sagittal plane. Due to one and/or all of these implications, on the rare occasion torque about the shoulder was calculated to be in the negative direction understood as extension $(C 2$ three left maximal mid stroke trials, $C 4$ and $C 3$ one left start stroke maximal trial, and $C 3$ one left submaximal mid stroke trial).

From Table 4.9, right limb dominance is prominent in all participants for both stroke styles. Again, an increase in torque is not necessarily a positive for injury prevention, however, torque applied in the opposite direction of the naturally predicted cycle should also be considered a key indicator for potential injury. Overall there was no difference between start and mid stroke torque about the respective mediolateral shoulder axis. 
Table 4.9 Estimated torque produced from human participants at peak impact

\begin{tabular}{|c|c|c|c|c|}
\hline \multirow{2}{*}{$\begin{array}{c}\mathbf{n}=6 \text { per } \\
\text { condition } \\
* \mathbf{n}=9\end{array}$} & \multicolumn{2}{|c|}{ Start Stroke } & \multicolumn{2}{|l|}{ Mid Stroke } \\
\hline & $\mathbf{L}$ & $\mathbf{R}$ & $\mathbf{L}$ & $\mathbf{R}$ \\
\hline$C 1(\mathrm{Nm})$ & $87 \pm 53$ & $2435 \pm 172$ & $192 \pm 59$ & $770 \pm 136$ \\
\hline$C 2(\mathrm{Nm})$ & $224 \pm 55$ & $1015 \pm 279$ & $-189 \pm 517 *$ & $600 \pm 280 *$ \\
\hline C3 $(\mathrm{Nm})$ & $18 \pm 240$ & $1248 \pm 482$ & $165 \pm 185$ & $1155 \pm 443$ \\
\hline$C 4(\mathrm{Nm})$ & $49 \pm 288$ & $997 \pm 166$ & $224 \pm 53$ & $1270 \pm 444$ \\
\hline$C 5(\mathrm{Nm})$ & $255 \pm 20$ & $1670 \pm 395$ & $370 \pm 146 *$ & $1610 \pm 633 *$ \\
\hline $\begin{array}{l}\text { Mean } \pm \\
S D(\mathrm{Nm})\end{array}$ & $134 \pm 197$ & $1079 \pm 522$ & $142 \pm 339$ & $1099 \pm 569$ \\
\hline
\end{tabular}

Similar to Section 3.4.4, impulse can be used to evaluate skating skill level in the sport of sledge hockey, specifically force transfer into forward skating. Increased impulse for skating in sledge hockey indicates either greater amounts of horizontal force are required to produce forward locomotion, or an increase in duration of the force application (increased pick-surface contact duration). As forward displacement is the desired outcome, an increase in vertical force is not necessarily a positive (less forward moving force). Adjusting the application of increased vertical forces (more horizontal) should promote improved forward displacement in turn improving skating output. Again, as skating is a continuous motion, increased impulse implies increased momentum, which can predict a decrease for skating introduced potential injuries. Results were calculated similar to Study 1 (Section 3.4.4). Overall, there was a slight difference between participant pick contact impulses. On average, left pick contact impulse ranged from $100 \mathrm{Ns} \pm 14 \mathrm{Ns}$ to $127 \mathrm{Ns} \pm 19 \mathrm{Ns}$, and right pick contact impulse ranged from $99 \mathrm{Ns} \pm 23 \mathrm{Ns}$ to 139 Ns \pm 21 Ns. 
Finally, skating velocity can be used as an indicator to evaluate skating skill level in sledge hockey. Overall, skating velocity ranged between $2 \mathrm{~m} / \mathrm{s}-6 \mathrm{~m} / \mathrm{s}$, with skating accelerations between $2 \mathrm{~m} / \mathrm{s}^{2}-12 \mathrm{~m} / \mathrm{s}^{2}$. Mean trial length for $C 1$ was determined to be almost $1.0 \mathrm{~s}$ longer than the quickest mean trial length from the topmost ranked participant within the tested population (C5). $C 5$ also had the shortest maximum trial length. As with all the above-mentioned key indicators, increase in velocity and acceleration may not be ideal from an injury prevention viewpoint. A longer and slower propulsion phase increases the length of time forces are applied to the load-bearing limbs, which can increase the potential for overuse/overloading injuries caused from skating. A shorter and quicker propulsion phase alters how the forces are applied to the point of rotation, potentially introducing an increase in torque related injuries. Further discussion regarding enhanced performance and injury prevention for skating in sledge hockey is presented in Chapter 5 .

\subsubsection{Estimated Limb Segment Peak Power at the Shoulder}

As previously stated, power can be used to compare work. Specifically in sledge hockey, limb segment power allows for the comparison of the rate at which skating occurs per limb. Power was directly affected by trial duration, start height, reaction force, and participant anthropometrics. Consistency within results was not expected. Limb segment specific power with respect to bodyweight (participant specific) was derived from the respective torques about the shoulders. Negative power is understood as extension of the limb segment. From Table 4.10, a mid stroke requires more power to maintain skating than the power required to start skating. Overall there was no difference between limbs for each respective stroke. An increase in overall skill level was proportional to an increase in power 
output.

Table 4.10 Estimated limb segment specific power produced from human participants at peak impact, normalized by bodyweight

\begin{tabular}{|c|c|c|c|c|}
\hline \multirow{2}{*}{$\begin{array}{c}\mathrm{n}=6 \text { per } \\
\text { condition } \\
* n=9\end{array}$} & \multicolumn{2}{|c|}{ Start Stroke } & \multicolumn{2}{|c|}{ Mid Stroke } \\
\hline & $\mathbf{L}$ & $\mathbf{R}$ & $\mathbf{L}$ & $\mathbf{R}$ \\
\hline C1 (W/kg) & $-19 \pm 12$ & $-18 \pm 14$ & $-65 \pm 40$ & $-54 \pm 14$ \\
\hline$C 2(\mathrm{~W} / \mathrm{kg})$ & $-44 \pm 21$ & $-40 \pm 21$ & $-36 \pm 26$ & $-26 \pm 22$ \\
\hline C3 (W/kg) & $-42 \pm 28$ & $-49 \pm 41$ & $-85 \pm 74$ & $-54 \pm 48$ \\
\hline$C 4(\mathrm{~W} / \mathrm{kg})$ & $-45 \pm 18$ & $-41 \pm 18$ & $-60 \pm 19$ & $-64 \pm 34$ \\
\hline C5 (W/kg) & $-95 \pm 37$ & $-130 \pm 66$ & $-139 \pm 84$ & $-150 \pm 84$ \\
\hline $\begin{array}{l}\text { Mean } \pm \\
S D(W / \mathbf{k g})\end{array}$ & $-49 \pm 36$ & $-51 \pm 47$ & $-79 \pm 65$ & $-72 \pm 65$ \\
\hline
\end{tabular}

\subsection{Discussion}

From a biomechanical perspective, Study 2 results suggest that the average male naïve sledge hockey player produces between $1.2-3.2$ times bodyweight peak impact force per arm during the downward poling stroke. Comparing $J R F_{\text {Shoulder }}$ and impact force, it can be deduced that an increase of approximately 0.2 times bodyweight of force is generated back onto each shoulder as a result of stick impact during skating in sledge hockey. Comparing with information presented in Section 2.1.3, peak impact force produced by naïve participants during downward poling in sledge hockey can be 5.1 times greater than the maximal force observed by Stoggl and Holmberg during elite upright double poling (475 N) [34]. From this, the suggestion that skating in sledge hockey does in fact introduce substantially increased impact forces compared to upright poling at force transfer initiation, is thus supported. From the above-mentioned, stick impact is predicted to be a potential mechanism of injury for the sledge hockey player. Potential injuries are predicted to be 
associated with soft-tissues overuse injuries such as muscle and tendon strains, and ligament sprains, as previously stated in Section 2.1.6. Increasing preventative practices are suggested to decrease overuse/overloading injuries to the upper limbs at impact.

Mechanically, force transfers throughout the propulsion phase, where a second much lower peak impact force is observed near push-off. Regardless of limb, effort, or stroke style, pick push-off forces were similar $(0.26-0.39$ times bodyweight $)$. In the naïve sledge hockey population (tested population), horizontal force was minimal compared to mediolateral force, which both were minimal compared to vertical impact force. From this, increased risk for potential vertical compression injuries at stick impact is probable. Again, these injuries are predicted to be associated with the soft connective tissues caused by repetitive impact. A majority of the participants produced heightened left limb forces during initial impact, which was succeeded by a diagonal force shift, where the right limb produced dominant forces at push-off (right-sided stagger). Minimizing this shift during skating is suggested to enhance individual skating skill level and performance output. Moreover, by minimizing the force shift during motion production, a decrease for potential asymmetrical injuries between the load-bearing limbs should occur. From Study 2 results, highly active naïve sledge hockey players producing a force shift during skating are likely to promote similar asymmetrical injuries as seen from the phenomenon observed in lower limb distance runners [26]. Force transfer imbalance from impact to push-off is predicted to only be observed in naïve and introductory sledge hockey players as a direct relation to their naivety with the motion and equipment. This suggests that participant comfort level with the motion and its respective equipment are proportional to enhanced skating skill 
level.

Within Study 2, three distinct stroking patterns were observed during the propulsion phase: 1) breaking, 2) propulsive, and 3) static held; the most popular pattern among the naïve participants was the pulling motion (breaking). It is predicted that expert sledge hockey players will demonstrate the pushing motion (propulsive) as their dominant stroking pattern. For all movements, extension occurred at the shoulder throughout pick contact. For the breaking pattern, the elbow flexed causing the forearm to move closer to the upper arm pulling the stick backwards past the original pick impact angle. From this, the potential for an increase in wrist movement is created. Future research involving dorsal hand markers can provide insight to exact wrist movement, and the effects within the skating motion. For the propulsive pattern, the elbow extended causing the forearm to move away from the upper arm pushing the stick forward, creating a more horizontal stick angle. Again, wrist motion is unclear; further wrist analysis in future research is suggested. For the final pattern observed during naïve skating in sledge hockey, static held stick, very minimal movement, if any, was observed from the entire limb-stick segment. From this, interpretation that the stick was used as a 'crutch' to assist in maintaining balance during motion can be made. This stroke pattern can be seen as a preventive technique to stop the body-sledge from falling over. This pattern is predicted not to be demonstrated from the expert population.

Upon review of pick impact angles, vertically aligned sticks at peak impact forces were observed for nearly all trials, for all participants. From this, in conjunction with spiking 
impact forces, and force generation at the shoulder, the potential for overuse soft-tissue injury as a result of repetitive stick impact is likely. Potential injuries would be parallel to similarly overloaded weight-bearing injuries seen in running, climbing stairs, standing up, jumping [26], [27], [36], [37]. Moreover, the importance of stroke mechanics has been established for the propulsion phase, not only to improve force transfer but also as a means to improve injury prevention involving the load-bearing limbs. Specifically, coaching staff should relay the importance of pushing throughout the propulsion phase compared to the common pulling motion observed from the naïve participants. The pulling motion was also observed in the study conducted by Hawkeswood involving community level sledge hockey players [19]. Substantial improvement is predicted to occur once the player masters the pushing technique. To enhance arm extension, coaches should also focus on improving comfort/balance during skating and torso flexion. Off-ice training can include the use of a bosu ball (ball up, Figure 4.15) in order to improve habituation with core balance while sitting on a narrowed gluteus-surface interface, mimicking the balance point within the sport of sledge hockey.

Mechanically, stroke initiation is suggested to occur above the shoulder ending with an extended forearm and flexed torso. Extending the elbow creates a more horizontal pick contact angle, both at impact and push-off, suggesting an increase in forward propulsion. Again, extending the elbow increases the lever arm, theoretically increasing torque about the shoulder. However, flexing the elbow promotes the pulling motion, which can produce torque opposite the natural cycle. Both motions should be considered key indicators for potential risk of torque related injuries caused from skating in sledge hockey. Again, 
increased torque increases stresses against the highly mobile shoulder joint, which may decrease stability within the sole locomotor for shoulder-dependent populations over repetitive use (see Section 2.2.2). From Section 1.1, 100\% of shoulder injuries in sledge hockey are sprains (tested population) [19]. Soft-tissue injuries can be interpreted to be related to torque. Chapter 5 discusses investigation of the lever arm (i.e., elbow angle) to enhance performance in conjunction with enhanced joint protection for sledge hockey players.

Overall, there was one superiorly skilled participant (C5), one bilateral participant (C4), and one participant who showed no improvement at all, lowest ranked (C2). From this, it can be inferred that participants with heighted upper limb athletic abilities, such as a wide receiver or hockey player, can adapt swiftly to the skating motion in the sport of sledge hockey. Furthermore, athletic ability in both limbs sets for an on-ice sport may provide increased athleticism improving bilateral stroke symmetry, specifically at stick impact. In contrast, upper limb athletic abilities, such as a wide receiver, can provide enough upper limb athleticism to successfully demonstrate the undesired pulling motion during skating in sledge hockey. Despite the consistent breaking poling motion, $C 3$ was ranked in the top half of the tested population. Upper limb athletic abilities, such as a linebacker, do not aid with skating performance skill level $(C 2)$. Results are consistent with a correlation between limb dominant athletic abilities and skating in sledge hockey. Positively correlated information involving upper limb dominant athletic abilities and skill level in sledge hockey can be used to guide newly classified shoulder-dependent $P w D s$ towards sledge hockey as a positive rehabilitative strategy. 


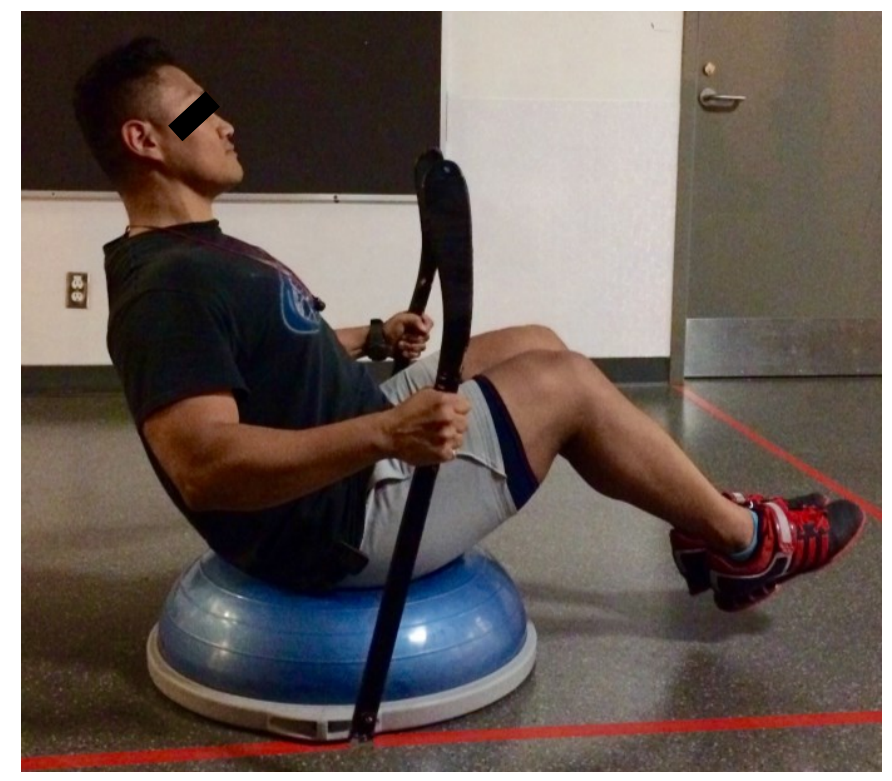

(a)

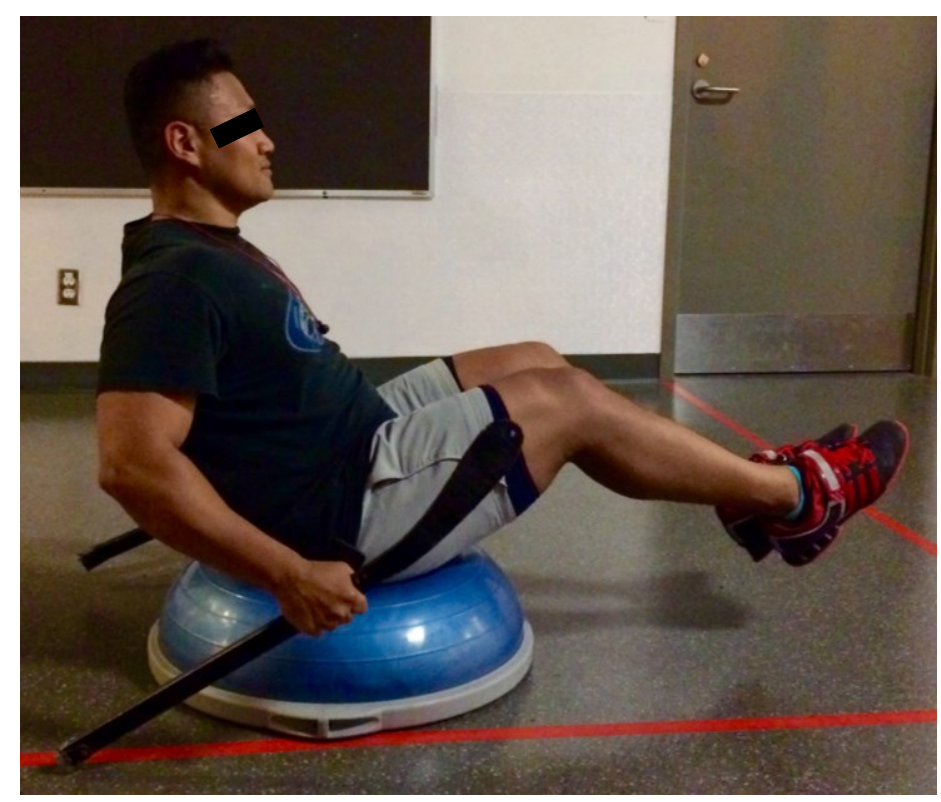

(b)

Figure 4.15 Bosu ball double poling off-ice training exercise to increase comfort within the sledge bucket improving balance during force transfer (a) Pick contact through to (b) Pick-off. 


\subsection{Conclusion}

Skating in sledge hockey can take different forms. Specifically, for the naïve population, a pulling motion was the prominent stroke pattern performed during double poling skating. This motion is not suggested as an ideal movement when concerned with forward poling weight-bearing locomotion. The pulling motion increases stick angle making force transfer less propulsive. The pushing motion was performed during a majority of the top ranked participant trials, resulting in an overall enhanced skating ability (tested population). Neither the start stroke nor the mid stroke can be concluded as the more difficult stroke style from the current tested population. Initial spiking impact reaction forces support the suggestion that stick impact can be a potential mechanism of injury.

Coincidently, both superiorly skilled participants ( $C 5$ and $C 3$ ), and the most inferiorly skilled participant $(C 2)$ with respect to overall skating skill level and the tested population, belong to the upper limb dominant sport of Canadian football.

Deduced information from Study 2 results can be used to promote engagement within the sport of sledge hockey, especially towards recently classified shoulder-dependent individuals. Suggested inferences include:

1) Upper limb dominant athletic abilities and strengths in task fluidity, balance, and positive habituation possessed prior to incident are predicted to promote swiftness in individual skill level growth.

2) On-ice both limb-set dominant athletic abilities may promote bilateral skating symmetry. 
3) Sport-specifically, players should focus training on improving balance and comfort within the sledge, especially during double poling forward skating.

4) Focus to attain a propulsive pick angle throughout the propulsion phase should be the upmost desired goal.

5) Off-ice training is recommended for prevention and rehabilitation, as any improvement concerning positive habituation may provide heightened protection from improper skating techniques. 


\section{Chapter: Aggregated Results}

This chapter consists of two sections. Section 5.1 discusses the results from Study 1 and Study 2 in a sport-specific context, specifically the skating motion. Section 5.2 discusses interpretations of the results from Study 1 and Study 2 with regards to potential injury introduced from skating in sledge hockey.

\subsection{Skating in Sledge Hockey}

Similar to heel-strike in walking, breaking forces are observed at impact within the anterior-posterior reaction force curves for the propulsion phase within skating for the sport

of sledge hockey (Figure 5.1 and Figure 5.2 middle). Moreover, multiple rapid alternating breaking-propulsive forces are observed within this portion of the propulsion phase. Overall, smoother waveforms are observed from higher skilled participants within the tested population, as well as from maximal effort tests. Decreasing impact breaking forces is predicted to improve forward acceleration. Increased fluidity during force transfer is interpreted from the smoother waveforms. Rapidly alternating breaking-propulsive forces are also sometimes observed during late propulsion, in what is interpreted to be preparation/initiation for push-off (Figure 5.1 middle).

Fluctuation between breaking and propulsive forces are interpreted to be a result of instability within the sledge itself throughout force transfer. Moreover, the minute surface contact area of the stick's picks implies decreased stability during motion compared to foot contact surface area. Sledge imbalance was observed from the static held stick during some of the propulsion phase results. Increased distance between the chassis is predicted to 
improve sledge-participant stability, however, decrease skating agility skill level.

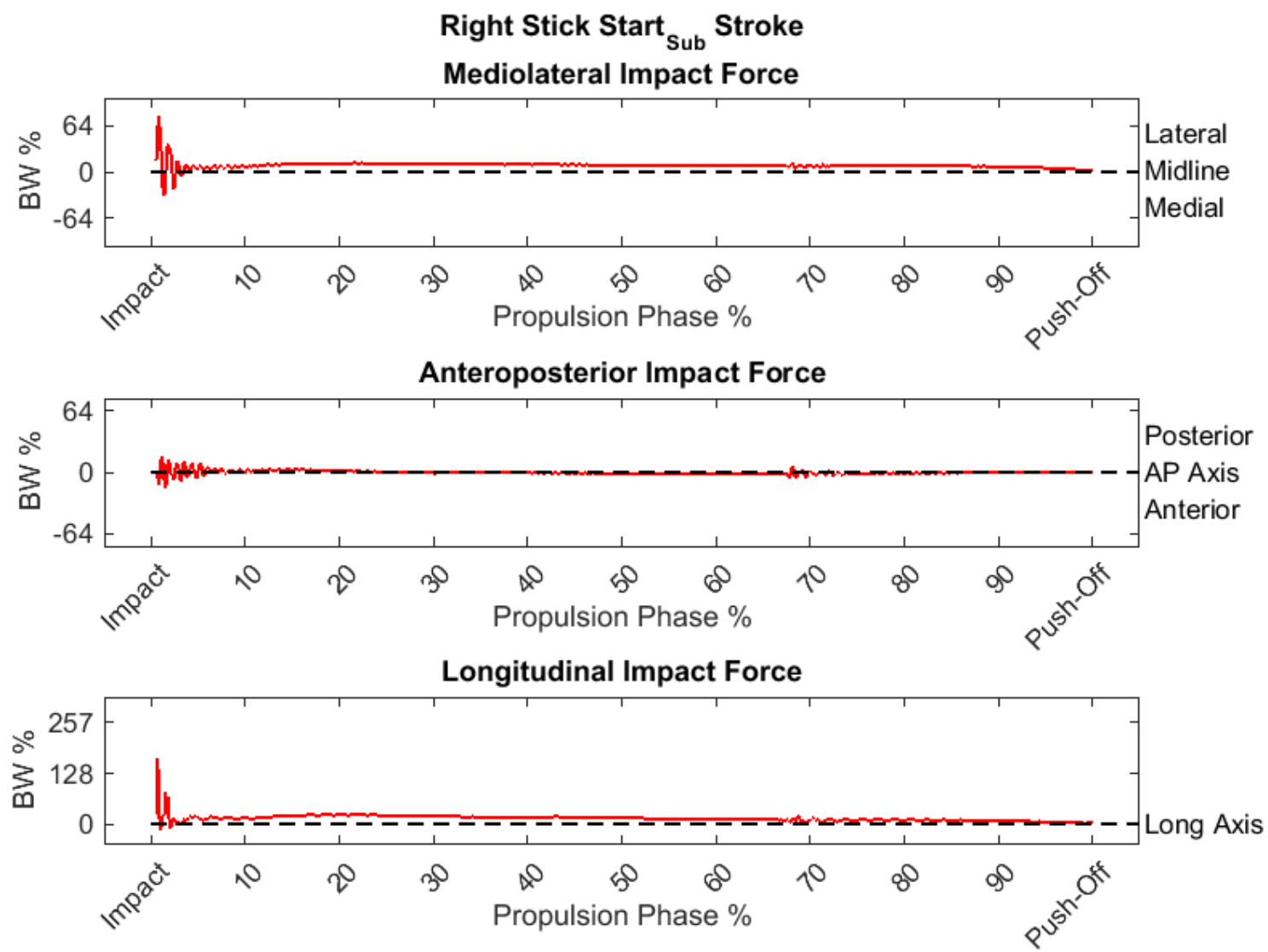

Figure 5.1 Right stick start stroke submaximal impact force distributions by bodyweight for the propulsion phase in the sport of sledge hockey. Mediolateral force curve (top), horizontal force curve (middle), and vertical force curve (bottom). Fluctuating breaking-propulsive forces are observed in the middle plot. Smoother waveforms are proportional to improved force transfer. 


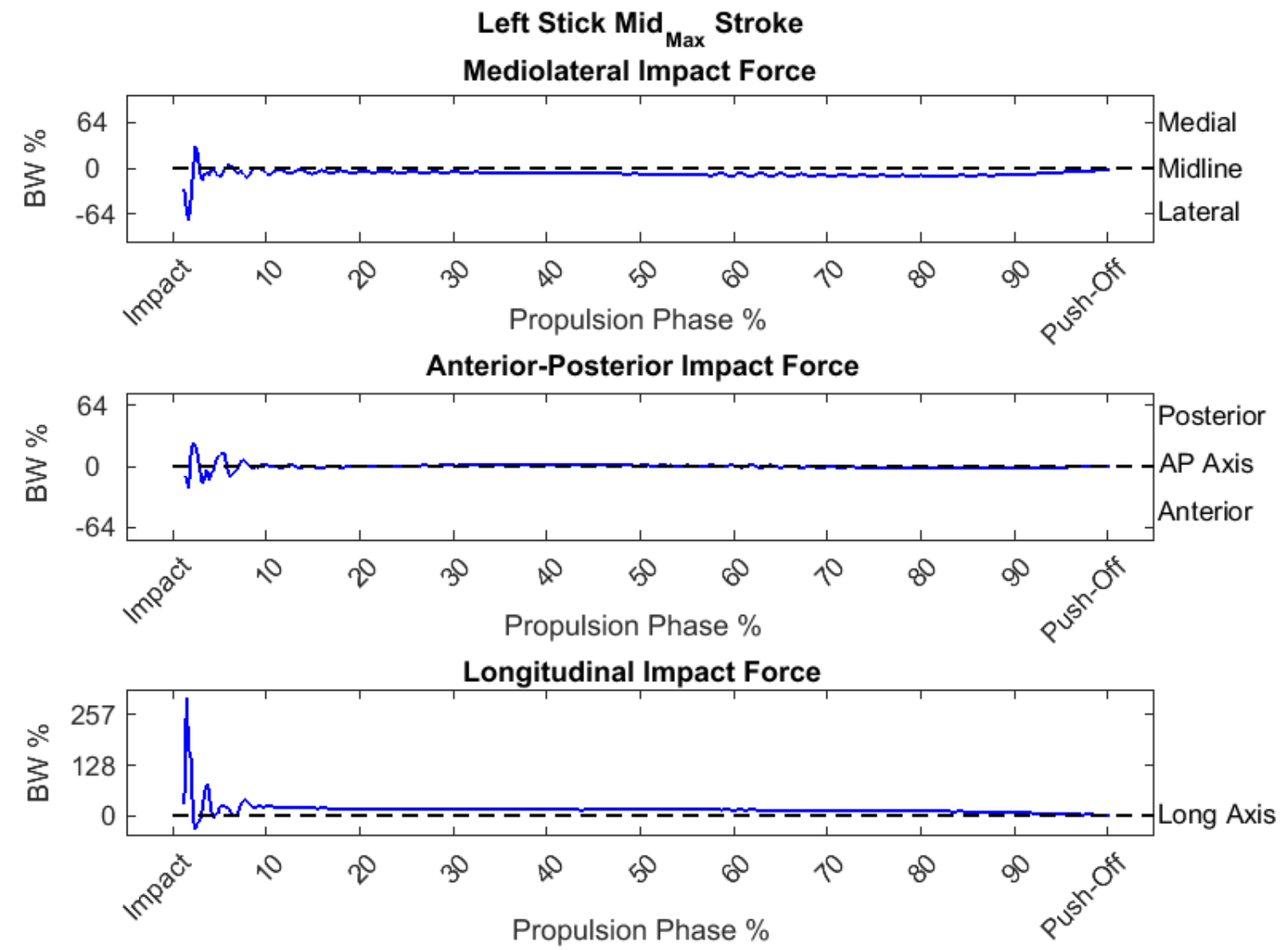

Figure 5.2 Left stick mid stroke maximal impact force distributions by bodyweight for the propulsion phase in the sport of sledge hockey. Mediolateral force curve (top), horizontal force curve (middle), and vertical force curve. Fluctuating breaking-propulsive forces occur less within the mid stroke (middle plot). From this, this participant produced enhanced force transfer compared to the submaximal start stroke; starting from static stop requires more force.

Impulse provides information regarding the amount of force required to produce movement or maintain continuous skating in sledge hockey. Rate of force development provides information regarding the limb's ability to create forward skating acceleration upon stick impact. Participants with an increase in upper limb athletic abilities demonstrated elevated impulse and rate of force development during skating tasks. From this, it can be inferred that improvement within any upper limb athletic ability should assist in improving a 
player's skating skill level in sledge hockey. This information broadens the range for training regimens that could be implemented for sledge hockey players, specifically diversifying the potential for off-ice training sessions. Furthermore, increased impulse implies increased momentum, which should decrease stresses onto the load-bearing limbs as the body-sledge should require less locomotive force to maintain motion. From this, support for improving skating skill levels concerning symmetry and fluidity within the stroking cycle can be made as a preventative measure.

As there was minimal differences between lever arm (i.e., elbow angle) and torque in Study 1, extending the elbow during the downward drop can be supported as means for improved stroke mechanics without increasing the risk of injury. Support for promoting elbow extension is provided from the more propulsive pick impact angles with the least increase in $J R F_{\text {Shouder }}$, and the slightly more efficient downward poling stroke that produces propulsive force. Moreover, from Study 2, an increase in resultant impact force was parallel with more propulsive impact angles, which were produced from top ranked participants. In Study 2, horizontal impact forces were minimal compared to vertical impact forces, which was perhaps due to the more vertically aligned sticks during skating. Due to the inconsistency of torque and power within Study 2, rehabilitative and preventative interpretations cannot be made with certainty, creating a gap between understanding the skating motion and its potential for injury.

Consistency and reliability can be provided through the use of a prototype. As the design of $S L A M-80$ resembles a sledge hockey player, a comparison between Study 1 and Study 
2 can occur. This comparison allows for the validation of $S L A M-80$ as a viable mechanical system when concerned with downward poling in the sport of sledge hockey. On average, participants in Study 2 were not able to achieve the baselines established from Study 1. Increased skill level was proportional with obtaining and/or surpassing the $S L A M-80$ baselines. Increased skill level was also proportional to a more propulsive pick plant. Increased effort was proportional with obtaining and/or surpassing the $S L A M-80$ baselines. The major limitation for Study 2 participant inability to produce baseline measures was perhaps due to the vertically aligned stick at impact. The vertically aligned stick may have been caused by a more vertical torso, implying that greater torso flexion promotes more horizontal stick angles, improving force transfer into locomotion. Since skating in sledge hockey can take many forms, standardized testing can become useful to establish initial guidelines/coaching techniques.

From restricted trials, the flexed elbow produced more upright pick plants compared to the extended elbow. A decrease in drop height was proportional to a decrease in impact force, with the odd horizontal force producing the highest impact force. As participant effort cannot be standardized, increase in impact force from shoulder level start heights were interpreted to be within the norm. Similar trends observed from the unrestricted trials were also observed in the restricted trials, specially left limb dominance at stick impact. Top ranked participants ( $C 5$ and $C 3$ ) were also able to obtain and/or surpass $S L A M-80$ baselines during restricted trials. Figure 5.3 provides a comparison of a mid elbow from $S L A M-80$ (blue) and mid elbow restricted trial (red) from Study 2. From this comparison, similar downward trajectories are observed enhancing the validation of 
$S L A M-80$ as a reliable prototype for sledge hockey impact analysis. Even though pushoff was not of concern for restricted trials, nearly all restricted trials for all participants indicated a pulling motion in order to produce movement.

From the evidence gathered between Study 1 and Study 2, SLAM - 80 can be viewed as a reliable and valid prototype to continue investigation of the downward pole in sledge hockey. Furthermore, SLAM - 80 is recommended in lieu of human participants during future investigations in order to minimize the addition of stresses onto the load-bearing limbs. The ability to investigate this newly identified potential for injury (stick impact) without introducing additional risk for injury is paramount in sledge hockey research. Variations of $S L A M-80$ are also recommended. Note: The same care should be implemented into the design of other models. 


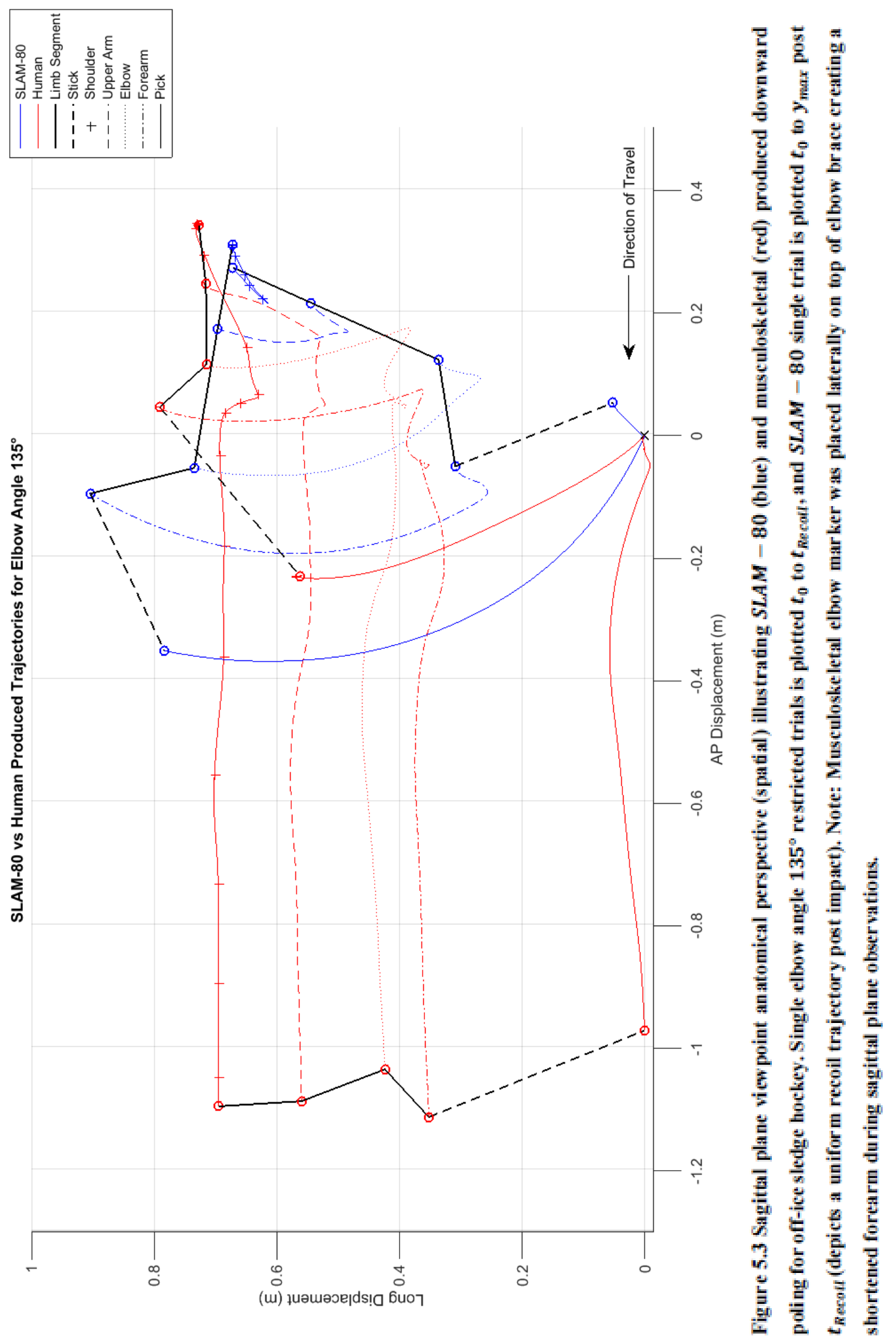




\subsection{Injury and Skating in Sledge Hockey}

The most common injury endured by wheelchair athletes involves the shoulder $(27 \%)$ [122]. Specifically, sledge hockey players endured 54\% of all reported injuries to the shoulder (26\%), and hand and wrist (28\%) [19]. Of all reported injuries, 31\% involved a sprain or injury to the soft-tissues of the shoulder, and 7\% a fracture to an upper limb bone [19]. Moreover, 2\% of all injuries reported were dislocations, 5\% fractures, $15 \%$ ligament sprains, and 21\% muscle strains [19]. From Study 2, hand injury can be presumed inevitable as more than on participant required Band-Aids to stop bleeding from scraped knuckles due to improper stroking techniques. Heightened awareness to hand location is suggested for coaching staff, especially with a naïve and introductory population.

Differences observed throughout naïve skating patterns in sledge hockey suggest altered applications of stresses against the shoulders. Axially loaded force can be less damaging than an off-axis (i.e., horizontal) force. Off-axis forces put tension on surrounding ligaments and tendons increasing the potential for injury. Specifically, torque determined about each shoulder was found to be different creating asymmetry during stroking, which can lead to an increase of potential torque related injuries caused from asymmetrical skating. Torque producing motion opposite the naturally predicted cycle could also increase potential for torque related injuries at the shoulders. Application of propulsion phase forces producing motion are suggested to be different than stresses applied against the hips during walking. The conjecture of increased lever arm at point of impact compared between walking and skating was presented in Section 2.2.2, identifying that moment arm affects how forces are applied to the point of rotation. A larger range of motion occurs from 
the shoulder joints compared to the hip joints during the gait cycle, introducing substantially elevated stresses applied at broadened maximal boundaries. This increase in angular range negatively (increase in stresses) affects how the load-bearing joints responded to these elevated reactions forces, in turn creating the need to further investigate impact forces produced by weight-bearing forward locomotion solely from the shoulders. From the above-mentioned, awareness for potential torque related risk of injury caused from skating is strongly suggested for all involved with sledge hockey, specifically awareness concerning lower skilled players. 


\section{Chapter: Conclusion}

This chapter summarizes the information and results presented in this thesis in a conclusive manner for skating in sledge hockey, and inferred potential for skating introduced injuries. Conclusions provide sport-specifics, preventive, and rehabilitative interpretations.

\subsection{Conclusion}

Skating within the sport of sledge hockey is suggested to be a motion in which elevated balance, force production, and ambidexterity are required for superior performance. Impact force from stick contact for naïve sledge hockey players was completed on a study-specific force plate design. On-ice equipment was modified in order to be used by all participants indoors. Sledge design is transferable to off-ice training in similar ways roller hockey and rolling skiing are to their respective sports; modifications are simple and can be implemented with ease.

Result are a first of its kind for this para-sport, and are used to initiate the development of a sledge hockey database for the purpose of athletic assessment, skill improvement, and sport-specific biomechanical analysis computer software. A prototype $(S L A M-80)$ is introduced and validated as an acceptable creator of free fall initial impact forces for downward poling in sledge hockey. SLAM - 80 is recommended in lieu of human participants allowing for continued investigation of skating initiation without introducing additional stresses onto the load-bearing limbs. 
Phase locations are defined with the relocation of the preparation phase prior to propulsion, opposed to the originally suggested swing phase majority. Phase relocation is supported through parallel analogies from hip gait, and double poling cross-country skiing, both upright and sit-ski. Further investigation involving the preparation phase and its initiation boundary is suggested to be a priority for future research.

Bilateral limb symmetry, balance within the stroking cycle and sledge, fluidity within stroking cycles, and positive habituation are recommended as key factors to produce enhanced locomotion during skating.

From results presented in this thesis, naïve skating in the sport of sledge hockey can be described as follows:

1. The skating motion can produce up to 3.1 times bodyweight of impact force at initial contact. Left limb mean impact force at maximal effort is $1.44 \times B W \pm$ $0.55 \times B W$ and $1.68 \times B W \pm 0.66 \times B W$, start and mid stroke respectively. Right limb mean impact force at maximal effort is $1.23 \times B W \pm$ $0.42 \times B W$ and $1.29 \times B W \pm 0.48 \times B W$, start and mid stroke respectively. Impact is identified as a potential mechanism for injury introduced from skating in sledge hockey.

2. The skating motion can produce up to 0.4 times bodyweight of reaction force at push-off. Focus on creating a pushing motion is suggested to improve skating at the naïve skill level (promoting a propulsive motion).

3. Limb dominance is observed for a majority of the tested population at impact 
and push-off; not necessarily the same limb was dominant at impact and pushoff.

4. An obvious lean and/or stagger opposite of the dominant impact limb occurs during the propulsion phase. Increased balance overall is suggested for enhanced skating.

5. An upright torso is suggested to promote increased pick impact angles. This is viewed as a disadvantage as propulsion phase stick angles were greater than the suggested propulsive range determined by $S L A M-80$.

6. Three distinct stick movement patterns were observed during the propulsion phase: 1) pushing (propulsive), 2) pulling (breaking), and 3) static held (increasing body-sledge balance). Guidance towards the pushing motion is suggested to occur for all skating skill levels.

In conclusion, enhanced locomotion in sledge hockey should promote heightened focus towards stroke cycle mechanics. Sledge hockey is suggested to be recommended as a rehabilitative athletic endeavour for recently classified shoulder-dependent individuals. Team play assists with coping and growth post traumatic events. Team play is especially helpful in rehabilitation practices for team-oriented professionals such as military personnel. Previous athletic superiority involving upper limb dominant sports promoting task fluidity, balance, and positive habitation are suggested as skill level enhancing assets for skating in sledge hockey. Skating in sledge hockey can take many forms, further investigation of all skating styles is recommended. This work is novel work within the area and begins to establish some of the foundation to build from for future research to work 
towards enhanced training/performance and injury prevention. 


\section{Chapter: Limitations and Amendments}

This chapter consists of three sections. Section 7.1 provides an outline of future research involving biomechanical analysis of skating in sledge hockey.

Section 7.2 provides limitations observed within Study 1 and Study 2 presented in this thesis.

Section 7.3 provides amendments as suggested by the limitations, specifically to the participant interview questionnaire and MOCAP marker system.

\subsection{Skating in Sledge Hockey, Potential for Injury, and Future Research}

From the results presented within this thesis, increased stresses involving the shoulders are predicted to be probable, both in an acute and chronic fashion. The $G H J$ is the most mobile joint in the human body, foreshadowing increased injury to the connective tissues of the shoulders from skating in sledge hockey. Spiking forces at impact increases the probable risk to the shoulders during repetitive skating. Moreover, injuries predicted from skating in sledge hockey are only the result of motion within sport. Sledge hockey is a full body contact sport where the shoulders are the most prominent aspect to the main body, increasing the probability of direct contacted upon body to body impact.

Improving stroke mechanics is determined to be the primary factor in order to produce enhanced skating in sledge hockey. Improved mechanics within each stroking cycle should result in a decrease of required locomotive force, in turning decreasing the probability of 
overuse and/or overloading injuries.

Future research concerning this research scope (sledge hockey skating biomechanics and injury) involves Study 2 and elite males, with the possibly of elite female sledge hockey players. Elite skill level is defined by a minimum of a provincial ranking within the sport of sledge hockey. Upon design of this thesis, elite female sledge hockey was not a prominent sport. Within the past few years, female involvement within the para-sport has become more publicly showcased prompting the need for investigation. Furthermore, future research aims to narrow the gap between $P w D s$ and skating biomechanics in sledge hockey, specifically involving military soldiers and/or veterans in rehabilitation. Participation in sledge hockey is strongly believed to be a positive socio-economic activity for $P w D s$, especially for team orientated professionals. Biomechanical investigations involving $P w D s$ broadens the current preliminary sledge hockey database. As observed in Study 2, the skating motion can take many forms, any evidence involving special populations provides improved understanding. Specially, investigations involving single amputees, double amputees, spinal cord injuries, or other physically disabling impairments can be useful for the current population of sledge hockey players (a similar classification systems were presented by Molik et al.) [58].

A proposed list of observable shoulder gait parameters for clinically appropriate assessment of skating within the sport of sledge hockey is provided. This list defined as the 12 Pointers is derived from a clinically appropriate guideline for routine observational gait analysis known as the 10 Pointers (Table 7.1) [35]. 
Briefly the 10 Pointers assess: 1) Step length asymmetry, 2) Ankle at contact (angle and heel contact location within the phase), 3) Knee angle at contact, 4) Stance phase knee flexion, 5) Single-limb support, 6) Ankle (angle range) and foot (limb loading during plantar flexion) during push-off, 7) Swing phase knee flexion, 8) Trunk angle, 9) Frontal plane: Trendelenberg sign (weak or paralyzed abductor muscles of the hip), and 10) Transverse plane: angle of patellae and feet, and arm posture [35].

\begin{tabular}{lll}
\multicolumn{2}{l}{ Table 7.1 Observable gait parameters } & Shoulder Gait \\
\hline Observable Parameter & Hip Gait & Unilateral Stroke Length \\
\hline \hline Asymmetry & Step Length & Bilateral Stroke Length \\
& & Unilateral Pick Angle \\
Contact & Ankle Angle & Unilateral Wrist Angle \\
& Knee Angle & Unilateral Elbow Angle \\
& & Extension Motion \\
Stance/Contact Phase & Knee Flexion & Single Limb \\
Support & Single Limb & Double Limb \\
& & Unilateral Pick Angle \\
Push-Off & Ankle & Unilateral Wrist Angle \\
& Foot & Bilateral Pick Angle \\
& & Bilateral Wrist Angle \\
& & Unilateral Elbow Flexion- \\
Swing Phase & Knee Flexion & Extension Motion \\
Trunk & Angle & Angle \\
\hline
\end{tabular}

Relevant parallel observable shoulder gait parameters are proposed as the 12 Pointers: 1a) Unilateral stroke length asymmetry b) Bilateral stroke length asymmetry, 2a) Unilateral pick angle at contact b) Unilateral wrist angle at contact, 3) Unilateral elbow angle at contact, 4) Contact phase unilateral elbow flexion-extension motion, 5a) Single limb support b) Double limb support, 6a) Unilateral pick and wrist angle during push-off, b) Bilateral pick and wrist angle during push-off, 7) Swing phase unilateral elbow flexion- 
extension motion, and 8) Trunk angle.

The 12 Pointers are suggested to used during future biomechanical analyses of skating in sledge hockey as an appropriate guideline for motion analysis. Continued improvement to the information developing the 12 Pointers will occur by the author.

Future investigations may concern:

1) Multiple stroking cycles including puck control in conjunction with on-ice inertial measurement units (IMUs) for both the pick of each stick, and sledge.

2) Artificial ice and $M O C A P$ - force plate $-s E M G$ system skating analysis

3) Roller sledge hockey and $M O C A P$ - force plate - $S E M G$ system skating analysis

4) Continued analysis of the start, and multiple mid strokes for double poling skating

5) Continued investigations involving $S L A M-80$ free fall downward poles involving:

1) Horizontal and above drop heights

2) Extended elbow ranges

3) Wrist-stick angle variations

4) Additional variations of $S L A M-80$ incorporating gender and various levels of body composition

6) Investigate stick material stress-strain properties in order to develop and/or modify pre-existing sledge hockey sticks. 
All research will relate back to improving rehabilitative and preventative practices for the sport of sledge hockey, and the shoulder-dependent population.

\subsection{Limitations}

Biomechanical analysis of skating in sledge hockey delivers a difficult task as MOCAP, and force plates truly only represent external values of the pendulum-like task. MOCAP and $S E M G$ data are acquired quantities from superficial skin locations leaving ample opportunities for artifacts to affect signal quality. Sagittal plane analysis produces slight inaccuracies compared to 3-dimensional data computations.

SLAM - 80 specific limitations included axel hole deformations introducing increased absorption within the wrist-stick and elbow joints. Figure 7.1 illustrates the effects of increased reaction forces from free fall downward poling onto the elbow joint. The elbow joint-centre axel was replaced prior to Study 1 investigations ( $~ 60$ trials). Implication of the effects of internal stresses to the elbow joint-centre are provided from the deformation observed within the joint-centre axel itself.

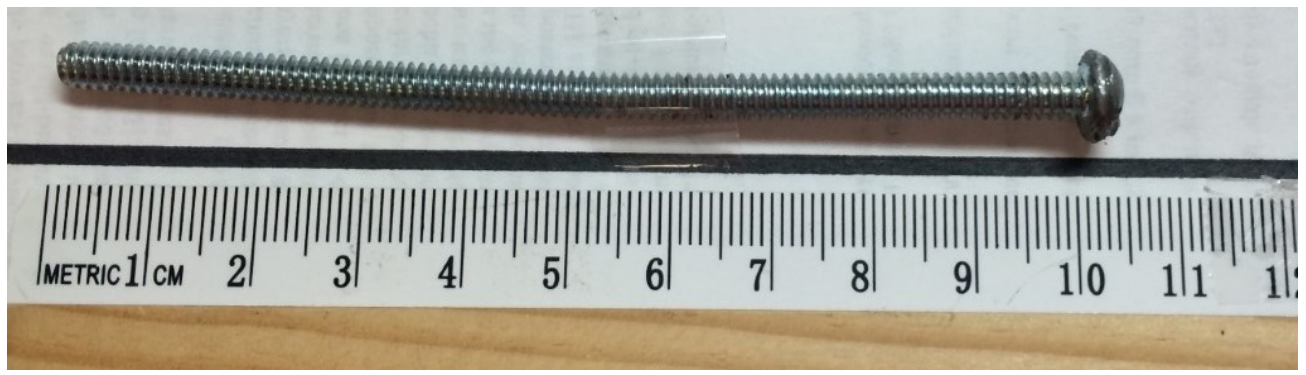

Figure 7.1 Elbow joint-centre axel screw deformation as a results of impact forces from approximately 60 downward skating poles. 


\subsection{Amendments}

Amendments to the Interview Questionnaire (Appendix 7.3D.8) have been made, specifically to lower limb anthropometric measurements: 1) exclusion of $A S I S$ - lateral malleolus, and replacement with $A S I S$ - lateral epicondyle (some participants may belong to the lower limb amputee special population), 2) addition of $A S I S$ - acromion sitting height, and 3) exclusion of muscle maximum voluntary isometric contraction (MVIC) table, replacement with $s E M G$ table. See Appendix E.1 yellow highlights for abovementioned amendments.

The addition of bilateral dorsal hand CoM markers has also been added to all future protocols allowing the computation of an appropriate wrist angle. Shoulder marker locations presented by Sholukha et al. have been added to all future protocols allowing heightened GHJ approximation [123]. A heightened vertebrae MOCAP tracking system is also suggested to be implemented in future research protocols in order to enhance torso angle analysis.

Shoulder-dependent population specific modified MVIC protocol is recommended to be developed and implemented, allowing for heightened investigation of in-vivo force generations within skating in sledge hockey. 


\section{Appendix A Background and Literature Review}

Appendices consist of four sections. Appendix A provides additional information in support for Chapter 2. Appendix B provides the blueprint for $S L A M-80$ structural design. Appendix $\mathrm{C}$ provides the mathematical models developed for inverse dynamic calculations and limb segment power. Appendix D provides documentations for participant recruitment and preparation as outlined in Chapter 4.

\section{A.1 Kinematic and Anatomical Terminology}

Kinematic analysis is developed from combining the global co-ordinate system with anatomical terminology [16], [67], [69], [96]. Biomechanically, forward-backward displacement along the anterior-posterior axis is represented by $x, y$ represents up-down displacement along the longitudinal axis, and $z$ represents left and right medial-lateral displacement from the midline along the mediolateral axis [16], [67], [69], [96]. Kinematic analysis is derived by the use of vectors, analogous to classic mechanics [16], [67], [69], [96]. Displacement co-ordinates are used to determine movement trajectories for investigative purposes [16], [67], [69], [96].

Since vectors can run through orthogonal axes within the 3-dimensional MOCAP capture area, the need to define the planes of motion arises. Anterior-posterior-mediolateral axes create the transverse plane dividing the body into top and bottom allowing for internal (towards midline) and external (away from midline) rotation [16], [67], [69], [96]. Anterior-posterior-longitudinal axes create the sagittal plane dividing the body into left and right allowing for abduction (outward movement) and adduction (inward movement) [16], [67], [69], [96]. Mediolateral-longitudinal axes create the coronal plane dividing the 
body into anterior (front) and posterior (back) allowing flexion (decrease in angle) or extension (increase in angle) [16], [67], [69], [96]. Movement past anatomical position creates hyper positioning [16], [67], [69], [96].

Measurement for each upper limb joint is standardized by the ISG with specific local coordinate orientations with respect to the reference co-ordinate [68]. GHJ movement analysis is based upon a Euler rotation sequence compared to the standard joint co-ordinate system established by Grood and Suntay used with other joints [68]. The adjustment to Euler decomposition for the $\mathrm{GHJ}$ measurement is required as the summation of flexion and abduction of the shoulder does not equal the summation of abduction and flexion [68]. For detailed joint angle methodologies see $\mathrm{Wu}$ et al.; thesis specific local co-ordinate locations are presented in Appendix $\mathrm{C}$ as part of the inverse dynamic proof [68].

Return to Kinematics Acquired Through Motion Capture.

\section{A.2 Kinetics Determined from Acquired Kinematics}

Biomechanical analysis uses kinematics to investigate Newton's Laws of Motion. From MOCAP data, velocity can be determined as a linear $(\Delta v)$ or angular $(\Delta \omega)$ measure. Newton's first law of motion, Law of Inertia, identifies that the resultant force $\left(\boldsymbol{F}_{\boldsymbol{\Sigma}}\right)$ (sum of all forces within the system) acting on an object in the state of uniform motion (constant velocity) must equal zero ( $\Delta v=0 \mathrm{~m} / \mathrm{s})$ [16], [67], [69], [96]. Force $\left(F_{i}\right)$ is represented as a Newton $(\mathrm{N})$, where one Newton is equivalent to one kilogram of mass $(m)$ moved at an acceleration of one metre per second squared [16], [67], [69], [96]. Acceleration ( $\Delta a$ and 
$\Delta \alpha$ ) investigates the rate of change of an object's velocity with respect to a time derivative $(\Delta t)$. Newton's second law of motion, Law of Acceleration, creates a relationship for an object's mass with its acceleration producing a resultant force with respect to an inertial reference frame, where angular rate must equal zero [16], [67], [69], [96]. Newton's third law of motion, Law of Action and Reaction, indicates that the action force $\left(\boldsymbol{F}_{\boldsymbol{i}-\boldsymbol{j}}\right)$ must equal the reaction force $\left(\boldsymbol{F}_{\boldsymbol{j}-\boldsymbol{i}}\right)$ in magnitude and opposite direction [16], [67], [69], [96]. Mathematical representation of Newton's Laws are presented in Appendix C as part of the inverse dynamic proof.

Energy is introduced in Section 2.2.2 demonstrating the evolution of kinetic evaluations into various forms of measurement. From this relationship, the first Law of Thermodynamics, the Law of Conservation of Energy arose indicating energy is a closed system [16], [96]. The second Law of Thermodynamics identifies the transformational properties of energy into these various forms with a distinct recognition for unusable energy known as entropy (no longer able to perform work); this transformation is known as transduction [67].

Since force applied over a distance produces work in Joules (J), a form of energy (ability to do work), and energy cannot be created nor destroyed, the Law of Conservation holds true [16], [96]. At a static position the upper limb's total energy measures $100 \%$ gravitational potential energy. This energy is transduced into kinetic energy as the limb arcs downward until midline (no potential energy) where the accumulation of kinetic energy provides enough work to return it to starting position (100\% potential energy). 
Return to Section Kinetics Acquired Through Motion Capture.

\section{A.3 Body Segment Inertial Forces}

Body segments have cylindrical shapes, therefore, a variation of the inertial equation for a slender rod can be used to derive the inertial effects at each CoM for each body segment (equations (A.1)); volume having the largest effect to the equation (equation (A.3)) [16], [96]. An object rotating about its own CoM or minimum radius produces a centroidal moment of inertia $\left(I_{0}\right)[16]$, [96]. Inertia for each segment can be mathematically calculated from standardized body segment proportions and lengths (equation (A.6)) [16], [96]. By the Parallel Axis Theorem, inertia can be determined for any arbitrary axis allowing segment inertia $\left(I_{i}\right)$ to be computed at various moments in time during dynamic motion (equation (A.5)) [16], [96]. Mass quantities for each body segment are also established from standardized body segment proportions [96]. The following provides mathematical proof of the above-mentioned as per Winter equation (4.3) through to equation (4.5), and equation (4.8) [96, p. 85-87, 89]:

Proof. Let $I=$ moment of inertia with a relation to its moment vector $(\boldsymbol{M}$, see equation (2.5))

$$
I=\sum_{i}^{n} m_{i} x_{i}^{2}
$$

where,

$x=$ distance from one end of the segment to the middle of the $i^{\text {th }}$ segment in metres, and $m_{i}=$ mass in kilograms of the $i^{\text {th }}$ segment.

Let $M=$ total mass of segment in Newton-metres

$$
M=\sum_{i=1}^{n} m_{i}
$$


where,

and

$$
m_{i}=V_{i} d_{i}
$$

$d_{i}=$ the density of the $i^{\text {th }}$ segment, which is assumed to be uniform throughout the segment creating a removable constant from the series, and $V_{i}=$ the volume of the $i^{t h}$ segment.

Net gravitational moment of force must create similar CoM distribution to the original mass distribution about any point along the segment axis. The CoM is located a distance of $x$ from the left edge of the segment. Adding $x$ to both sides of equation (A.2), and solving for $x$, we get

$$
x=\frac{1}{M} \sum_{i=1}^{n} m_{i} x_{i}
$$

a representation of the complex mass distribution in a single quantity $(M)$ with a $\operatorname{CoM} x$ distance from one edge of the segment. Each segment within a multi-segment system has a $\operatorname{CoM}_{i}$; the entire linked system has a $\operatorname{CoM}_{0}$. Equation (A.4) is used to find the $x_{0}$ and $y_{0}$ co-ordinate locations.

From the relation presented in equation (2.5) and equation (A.4), equation (A.1) becomes

$$
I=I_{0}+m x^{2}
$$

and

$$
I_{0}=m \rho_{0}^{2}
$$

where, 
$\rho=$ radius of gyration in metres, as per Winter equation (4.9) and equation (4.10) [96].

From Newton's Second Law and the theory of Conservative Force, a relationship exists between force and acceleration, where acceleration is the derivative of velocity (see equation (A.7)) [16], [96]. Since momentum has a relationship with an object's velocity, a relationship with force can be derived with respect to a time derivative (equation (A.8)) [16], [96].

$$
\begin{gathered}
\int_{i}^{n} F d t=m v_{n}-m v_{i} \\
\sum \boldsymbol{F}=d \boldsymbol{p} / d t
\end{gathered}
$$

where,

$$
p=\text { linear momentum in kilogram-metre per second. }
$$

Examination of the integral in equation (A.7) reveals a representation of liner impulse $(J)$ (product of the force applied onto an object and the duration of force applied) in Newtonseconds [16], [96]. This relationship creates the Momentum-Impulse Theorem, which states that the change in an object's momentum must equal the impulse applied to it (equations (A.9) and (A.11)) [16], [96]. As an angular $(L)$ quantity, momentum uses the relationship of angular acceleration and velocity in the same manner, creating a relation with the system's moment (equations (A.10) and (A.12)) [16].

$$
\begin{gathered}
\boldsymbol{M}=\boldsymbol{r} \times d \boldsymbol{p} / d t \\
\boldsymbol{L}=\boldsymbol{r} \times(m \boldsymbol{v})
\end{gathered}
$$




$$
\begin{gathered}
\boldsymbol{M}=d \boldsymbol{L} / d t \\
L=I \omega
\end{gathered}
$$

where,

$$
\boldsymbol{r}=\text { position vector }
$$

In biomechanics energy is measured in power, specifically total segment power (limbsegment power in this thesis) [16], [35], [67], [96]. Power, the rate of doing work relates the flow of energy from joint to joint through force, moment, and velocity (equation (A.13)) [35], [67], [96]. From this, production power for a linked system can be understood through the summation of passive (force and velocity representing joint transfer energy) and active (moment and angular velocity representing muscular contribution involving the linking joints) power flow. Further mathematical representation of limb segment power is presented in Appendix C.2.

$$
P=F \cdot v+M \omega
$$

Return to Gravitational Energy on a Dynamic System.

\section{A.4 Signals Acquisition and Processing}

Frequency $(f)$ measures the oscillation rate of the harmonic signal in cycles per second (Hertz (Hz)). One cycle per second is equivalent to one Hertz (equation (A.14)). Frequency is one of four characteristics for a signal: 2) amplitude (a) quantifies the magnitude of the signal, 3) DC offset $\left(a_{0}\right)$ (direct current bias) represents the average value of the signal, and 4) the phase angle $(\theta)$ or shift in signal indicates the delay or time-shift within the signal [96], [124]. Time in seconds, as a discrete value relates inversely to the sample rate. This 
is a useful conversion as sample rates differ between the various systems. Conversion into a time domain allows for simultaneously acquired data to be integrated. Moreover, a common time domain allows for comparison between acquired signals.

$$
f=1 \text { cycle } / t=\mathrm{Hz}
$$

Fourier analysis extracts sine and cosine functions from a series, where when superimposed the function is reproduced [96], [124]. Fourier transform decomposes a signal into the frequencies that make it up [96], [124]. Fast Fourier transform $(F F T)$ is an algorithm that is used to compute the discrete Fourier transform of a sequence (or its inverse) [96], [124]. FFT transfers a function of the time domain into a function of the frequency domain [96], [124]. The Nyquist Theorem states that in order to reproduce an acquired analog signal, as a digital quantity, the sampling rate must be twice that of the highest frequency [96], [124]. The Nyquist sampling frequency is the lowest sampling rate a signal can be acquired from. Shannon's Reconstruction Formula is used to provide a truer representation of undersampled signals (equation (A.15) [96], [124]. Current biological signal acquisition technology should exclude the option to under-sample a signal, as memory storage is exponentially greater than preliminary signal investigations.

$$
\begin{gathered}
h(t)=a_{0}+a \sin (\omega t+\theta) \\
\omega=2 \pi f
\end{gathered}
$$

SNR is the strength of a signal generally expressed in decibels (dB) (equation (A.18)) [96], [124]. SNR is the power of a signal over the power of noise or unwanted signal distortion (equation (A.17)) [96], [124].

$$
S N R=\frac{P_{S}}{P_{N}}
$$




$$
\operatorname{SNR}(\mathrm{dB})=10 \log \frac{P_{S}}{P_{N}}
$$

Return to Signal Acquisition and Processing.

Return to Processing and Publication of Acquired Signals.

\section{Appendix B}

\section{B.1 SLAM - 80 Blueprint}
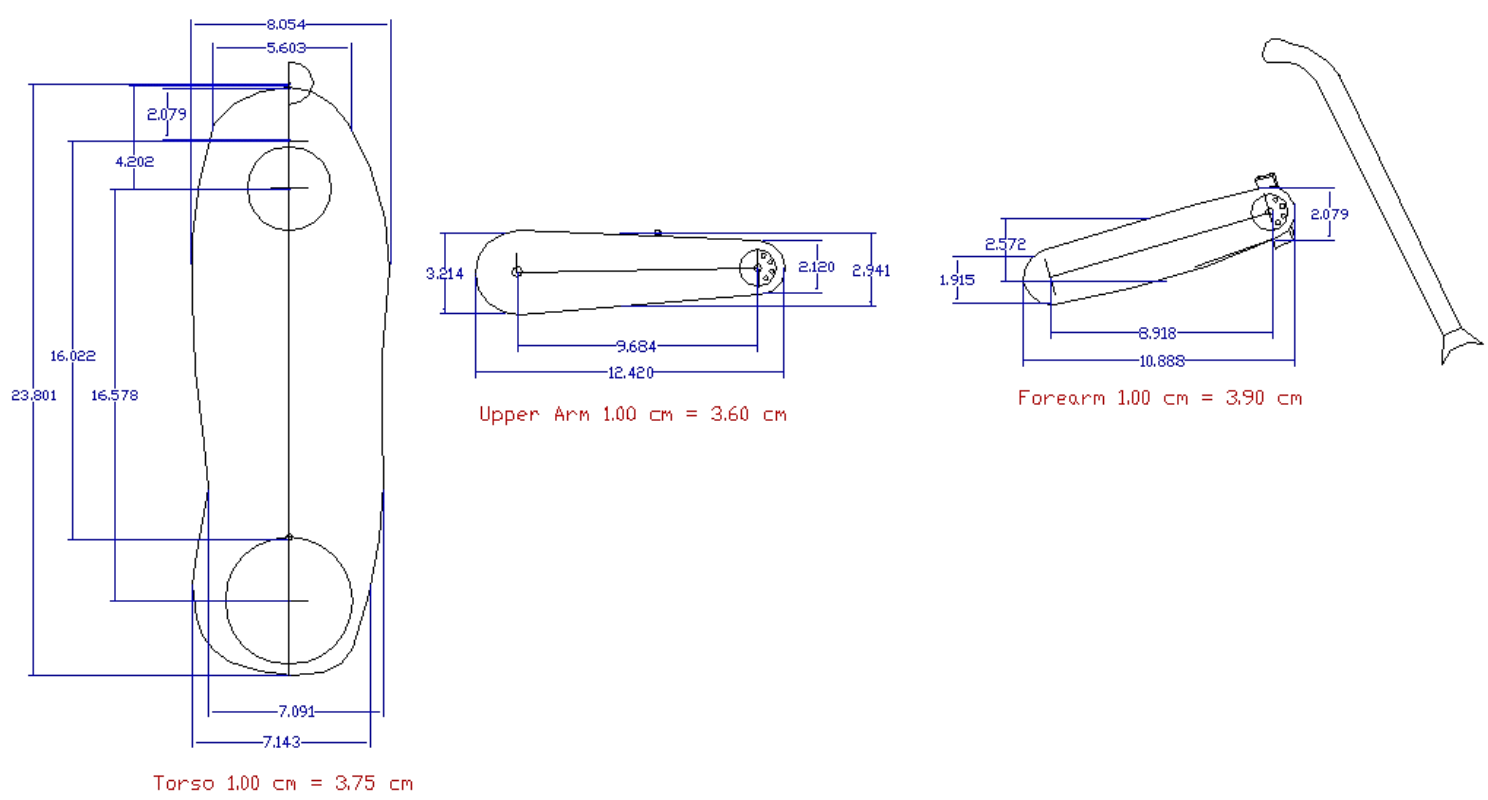

Return to Segment Architecture \& Morphology. 


\section{Appendix C Theoretical Proofs - Mathematical Models}

\section{C.1 Inverse Dynamics Model for the Shoulder in the Sport of Sledge Hockey}

Model C.1 Inverse dynamics model for the shoulder in the sport of sledge hockey as per Winter [96]:

Sagittal plane motion excludes movement along $\pm z-$ axis. The subsequent theoretical model is developed for the sagittal plane $(x, y)$. Let $\mathbf{e}_{\mathbf{1}}$ and $\mathbf{e}_{\mathbf{2}}$ represent the unit vectors along the co-ordinate axes for this mathematical model. Let $\mathbf{e}_{\mathbf{1}}$ represent the anterior-posterior axis allowing forward-backward displacement, and $\mathbf{e}_{2}$ represent the longitudinal axis allowing up-down displacement. Combined they create the sagittal plane allowing motion producing flexion and extension from the shoulder and elbow, and radial and ulnar deviation from the wrist in a neutral forearm.

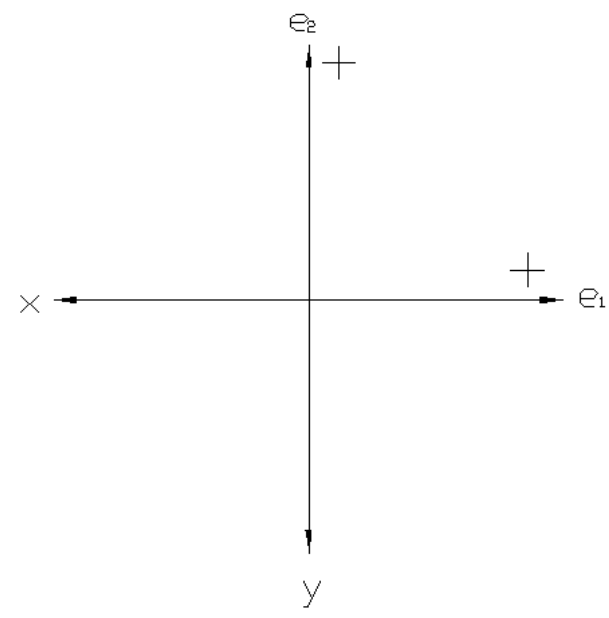

Figure C.1 Sagittal plane co-ordinates system for this model used to investigate skating in sledge hockey.

Let $\boldsymbol{A}$ represent the vector under investigation where

$$
\boldsymbol{A}=x_{i} \mathbf{e}_{1}+y_{i} \mathbf{e}_{2}
$$

with a point

$$
A\left(x_{i}, y_{i}\right)
$$

and magnitude 


$$
\|A\|=\left(x_{i}^{2}+y_{i}^{2}\right)^{1 / 2}
$$

Let $\boldsymbol{A}^{\circ}$ represent the joint-centre vector under investigation.

Let the distance between two points be a manipulation of equation (C.3)

$$
\|A\|=\left(\Delta x^{2}+\Delta y^{2}\right)^{1 / 2}
$$

Let $d$ represent length in metres, and $\theta$ angle in degrees $\left(^{\circ}\right)$ of $d$, where $\theta$ is the measured angle (ъ) between $d$ and $\mathbf{e}_{\mathbf{1}}$ (Figure C.2).

$$
\begin{aligned}
& x_{i}=d \cos \theta \\
& y_{i}=d \sin \theta
\end{aligned}
$$

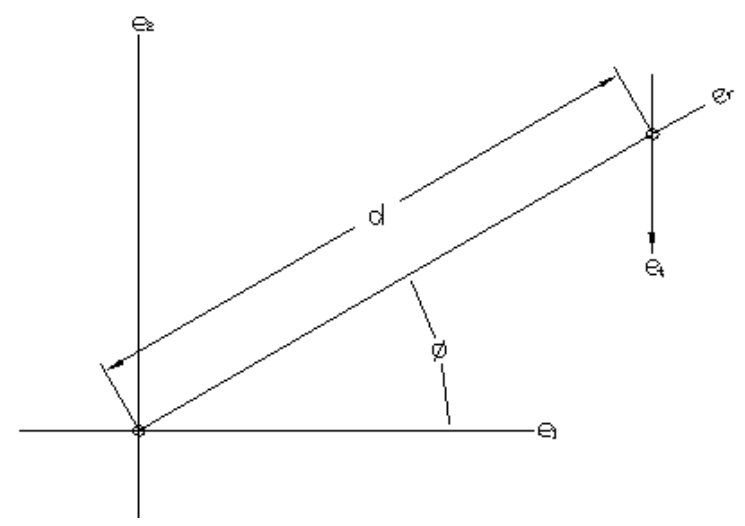

Figure C.2 Sagittal plane angle measurement definition with regards to the vector under investigation; angle measures $\mathrm{e}_{1}$ counter clockwise to $\mathrm{e}_{2}\left(0^{\circ}-90^{\circ}\right)$.

From $I S G$ standards, upper arm segment alignment relates the GHJ local co-ordinate with the forearm origin co-ordinate (Figure C.3). Specifically, the GHJ $y$-axis runs along the long-axis of the humerus from the midpoint of the lateral and medial epicondyle points to the GHJ-centre [68]. Then, the $z-$ axis is created from the line perpendicular to the plane created from $y$-axis of the humerus and $y$-axis of the elbow, pointing to the right [68]. Finally, the $x$-axis is created from the common line perpendicular to the established $z-$ axis and $y-$ axis, pointing forward [68]. 


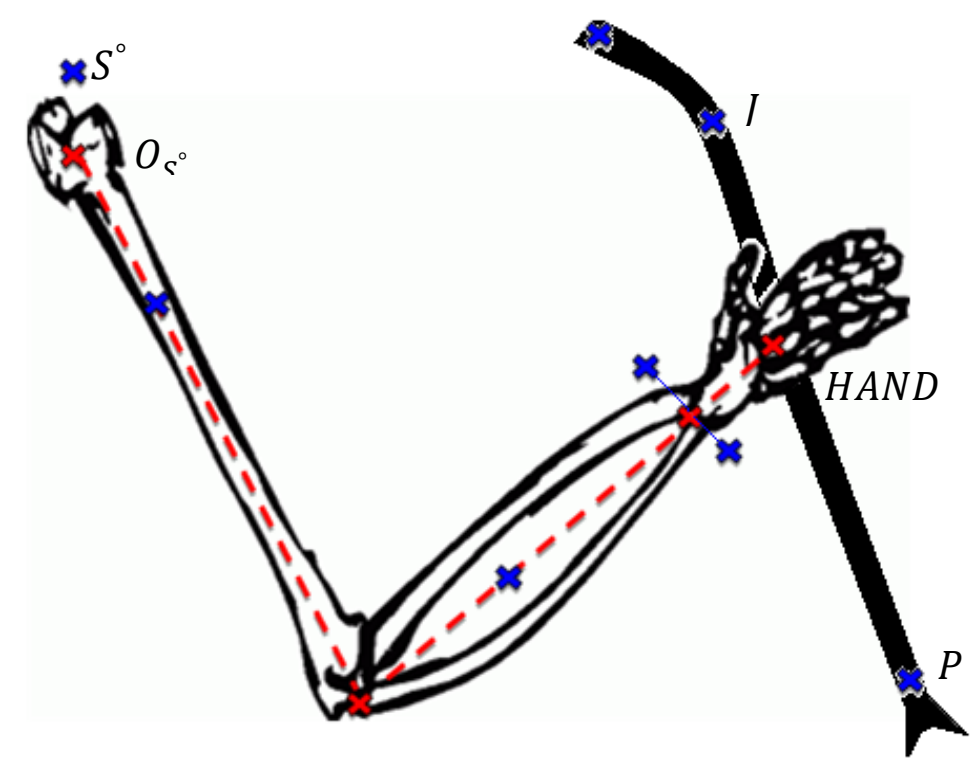

Figure C.3 Sagittal plane multi-segment alignment used in this model to investigate skating in sledge hockey; human participant marker location (blue), and joint location for calculations (red). Forearm and wrist are in neutral rotation in order to produce locomotion from the stick [68]. Markers outlined are required for below-mentioned manipulations; pick of stick $(P)$, joint of stick $(J)$, palm-stick interface $(H A N D)$, shoulder $\left(S^{\circ}\right)$, and $G H J$ local origin $\left(O_{S^{\circ}}\right)$. Markers along the long axes are used in calculations for both studies.

In sledge hockey a neutral forearm and wrist (thumb up) occurs allowing the use of the sticks to produce motion (Figure C.3). Study 1 did not require manipulations among the marker trajectories as marker locations were at exact joint-centres and pre-established CoMs. Study 2, had limitations due to the inability to directly track in-vivo motion creating the need to manipulate some acquired marker locations. Additional marker locations are suggested below, and aggregated in Section 7.3 and Appendix E to allow more precise computations.

In Figure C.3, human participant marker locations are represented in blue. Joint locations for calculations are represented in red as per the abovementioned upper limb alignment [68]. CoM markers did not require 
manipulation for sagittal plane computations. In human participants, the shoulder marker was required to be manipulated to represent the GHJ. A normal acromiohumeral interval ranges from $9-10 \mathrm{~mm}$ with an average male humeral head diameter of $49 \mathrm{~mm}$ [125], [126]. Therefore, a negative $y$ trajectory shift for the shoulder marker is determined to be $43 \mathrm{~mm}$ as a standard displacement for each participant at rest, upright, and upper arm in anatomical position (displacement incorporates marker diameter, bone acromion thickness $(7.7 \mathrm{~mm})$, and the assumption shoulder marker is located properly on the acromion) [127]. From this, a relation between the shoulder, GHJ, and the upper arm CoM can be made and used as a reference. Future research is suggested to follow marker locations presented by Sholukha et al. for heightened GHJ approximation [123].

In a static position, sitting upright, with the upper arm in anatomical position, using equations (C.2) and (C.3), respectively, and the abovementioned information, we get point,

$$
G H J^{\circ}\left(S_{x}^{\circ}, S_{y}^{\circ}-0.043 \mathrm{~m}\right)
$$

with a magnitude of

$$
\|A\|=0.043 \mathrm{~m}
$$

from the shoulder marker to the $G H J$-centre.

We know the length of the upper arm (pre-measured) ( $U A=$ upper arm length in metres), so we get,

$$
\|B\|=U A-\|A\|
$$

where, the distance from the upper arm $\operatorname{CoM}(u a)$ to the GHJ-centre is a constant ( $B$, bone length does not change).

From properties of a right angle triangle, the angle of the humerus can be found from the elbow marker and upper arm marker $(C)$. From equations (C.4), (C.2), and (C.3), respectively, we get,

$$
\|C\|=\left(\Delta x^{2}+\Delta y^{2}\right)^{1 / 2}
$$


with right angle corner point

$$
D\left(u a_{x}, E_{y}^{\circ}\right)
$$

and magnitude

$$
\|D\|=\left(\Delta x^{2}+\Delta y^{2}\right)^{1 / 2}
$$

From trigonometry of a right angle triangle, we get,

$$
\alpha=\cos ^{-1}(\|D\| /\|C\|)
$$

Then from equations (C.5) and (C.6), we get,

$$
\begin{aligned}
& x_{G H J^{\circ}}=x_{u a} \pm B \cos \alpha \\
& y_{G H J^{\circ}}=y_{u a}+B \sin \alpha
\end{aligned}
$$

where the direction of $x$ is dependent upon elbow location with respect to the $G H J$.

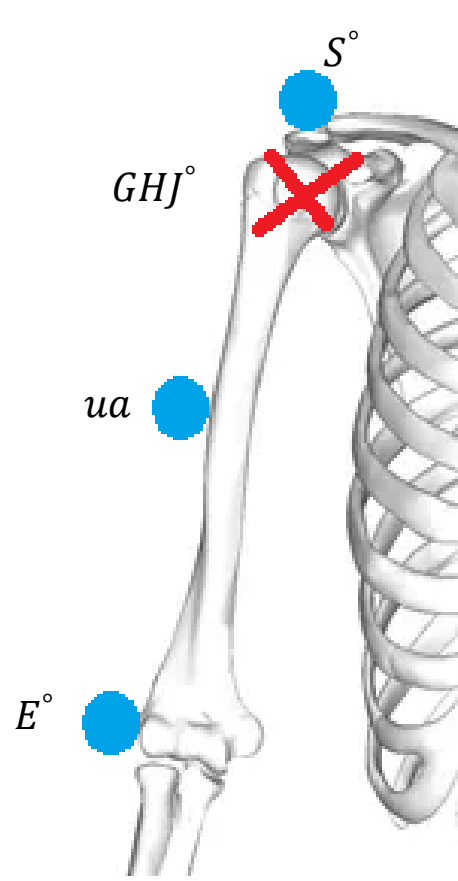

(a)

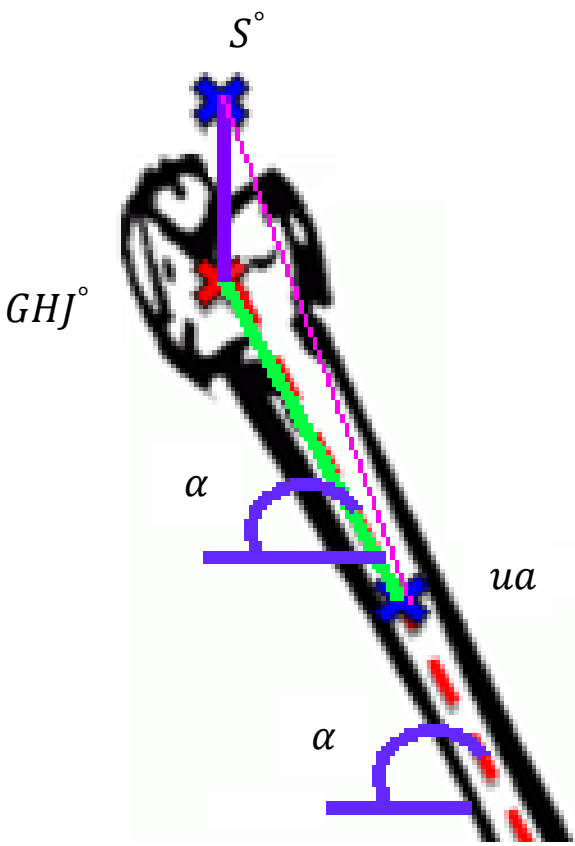

(b)

Figure C.4 (a) Human participant marker locations for the upper limb (blue circles), and GHJ-centre (red $x)$, and (b) Sagittal plane shoulder marker $\left(S^{\circ}\right), G H J^{\circ}$, and upper arm CoM (ua) relationship for shoulder marker manipulations. The distance from upper arm marker to $G H J^{\circ}$ is a constant (green line), and the angle of the humerus can be calculated $(\alpha)$ from the elbow marker to the upper am marker. Then by trigonometry $G H J^{\circ}$ co-ordinates can be found $(\operatorname{red} x)$. 
In human participants, wrist joint-centre was computed as the midpoint between the connected wrist markers. In human participants, the palm-stick interface was required for calculations. A dorsal hand marker at this location is suggested for future researches. From Table 4.1 in Winter, hand CoM is $0.506 \cdot H A N D$ from the proximal end, where the average male hand is $0.1938 \mathrm{~m}$ [20], [21], [96, p. 86]. From Plagenhoef et al. the average male hand CoM is $0.468 \cdot H A N D$ from the proximal end [128]. Since the hand is in a fist when gripping a sledge hockey stick, CoM would be slightly closer to the wrist than when fingers are extended. From this, Plagenhoef CoM will be used as the most available peer-reviewed CoM percentage. Let $H A N D$ represent hand CoM at $\cap$ stick shaft, where wrist to hand distance is $0.0907 \mathrm{~m}$ in anatomical position. We also know the pick and joint marker locations at the distal and proximal ends of the stick shaft, respectively.

With a straight forearm and wrist, and a $90^{\circ}$ stick angle to the forearm, we can use the properties of a right angle triangle, the magnitude of the wrist joint-centre to joint of stick can be found from marker locations $(C)$. From equation (C.3), we get,

$$
\|C\|=\left(\Delta x^{2}+\Delta y^{2}\right)^{1 / 2}
$$

Then by Pythagorean's Theorem, we get,

where,

$$
A=\left(C^{2}-0.0907^{2}\right)^{1 / 2}
$$

$$
D=S T K-A
$$

and $S T K$ is the distance from stick joint to pick marker, making $A$ and $D$ constants with a static hand-stick interface (hand does not move up and down the shaft).

From Law of Cosine, $A=0.0907 \mathrm{~m}$, constant $B$, and $\left\|C_{i}\right\|$ we find,

$$
\begin{gathered}
\alpha=\measuredangle W^{\circ} \text { HANDJ } \\
\alpha_{i}=\cos ^{-1}\left(C_{i}^{2}-\left(B^{2}+A^{2}\right)+2 B A\right)
\end{gathered}
$$

where, $\alpha$ is the angle opposite the hand-stick angle $(\tau)$ 


$$
180^{\circ}-\alpha=\tau
$$

Note: Depending on how close the hand-stick interface is to the joint marker, variable manipulation may be required for $\alpha_{i}$.

The same process can occur using constant $D$, where $\gamma$ is internal wrist angle to the forearm where $>180^{\circ}$ is ulnar deviation.

Then from the angle of the stick $(\eta) \operatorname{HAND}(x, y)$ can be found. From equations (C.5) and (C.6), we get,

$$
\begin{aligned}
& x_{H A N D}=x_{W^{\circ}} \pm A \cos \eta \\
& y_{H A N D}=y_{W^{\circ}} \pm A \sin \eta
\end{aligned}
$$

where the directions of $x$ and $y$ are dependent upon $H A N D$ location with respect to the wrist joint-centre.

We know stick $(S T)$ shaft segment length is $0.5500 \mathrm{~m}$ including the picks, and width is $0.0300 \mathrm{~m}$. Blade $(B L)$ segment length is $0.2100 \mathrm{~m}$ at a $10^{\circ}$ curve angle, and width of $0.0550 \mathrm{~m}$. We can assume simple geometry for the sledge hockey stick: 1) rectangular shaft (Figure C.6 green), and 2) rectangular and triangular blade (Figure C.6 red). From this, and equations for $C o M$ and inertia developed through classic mechanics, we can solve for stick CoM (Figure C.6 yellow x), and radius of gyration [16], [17]. We also know wrist-pick length is $0.4000 \mathrm{~m}$ for the $S L A M-80$ sledge hockey stick. Human participant stick dimensions are outlined in Section 4.3.1. 


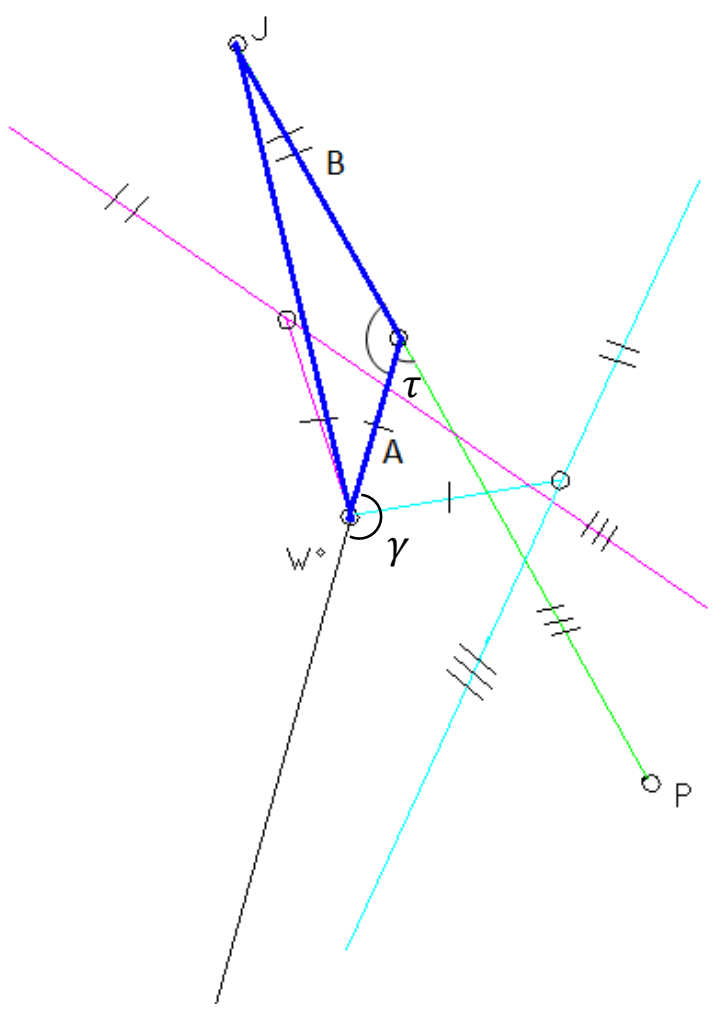

Figure C.5 Sagittal plane representation of various random wrist angles; magenta - neutral wrist radial deviation, cyan - neutral wrist ulnar deviation, and green neutral wrist along the vector created by the forearm. The distance from wrist to hand $\operatorname{CoM}$ is represented by $\mathrm{A}$, assuming the hand-stick interface remains (hand does not move up and down the shaft). The distance of hand CoM to joint marker $(J)$ is represented by $B$, again assuming the hand-stick interface remains. From these relations the final side to the triangle can be solved, and interior angles determined via the Law of Cosine or Sine Law (blue triangle). $\tau$ is wrist-stick angle, and $\gamma$ wrist angle with respect to the forearm.

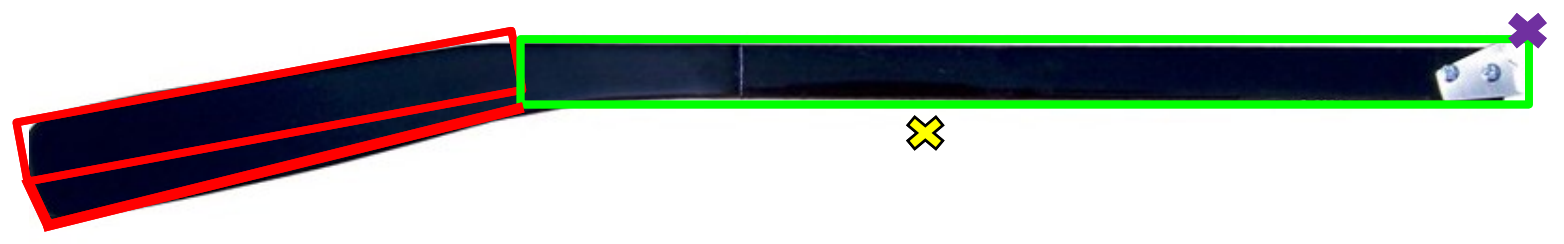

Figure C.6 Assumed geometric shapes of a typical sledge hockey stick. CoM (yellow x) and radius of gyration for a sledge hockey stick can be solved using the outlined geometry. Assume rectangular widths equal $\left(b_{\text {red }}=b_{\text {green }}\right.$, where $b$ represents base) and local origin $(0,0)$ is at the purple $x$. 
Let $s t$ represent stick CoM. From classic mechanics area (A), and shape CoM inertia $\left(I_{0}\right)$ and radius of gyration $\left(\rho_{0}\right)$ for a triangle are [17]:

$$
\begin{gathered}
\mathrm{A}=b(h / 2) \\
I_{0}=b\left(h^{3} / 36\right) \\
\rho_{0}=h / \sqrt{18}
\end{gathered}
$$

From classic mechanics area (A), and shape $\operatorname{CoM}$ inertia $\left(I_{\mathbf{0}}\right)$ and radius of gyration $\left(\rho_{0}\right)$ for a rectangle are [17]:

$$
\begin{gathered}
\mathrm{A}=b h \\
I_{0}=b\left(h^{3} / 36\right) \\
\rho_{0}=h / \sqrt{12}
\end{gathered}
$$

From this, Table C. 1 and Table C. 2 can be created, where $b_{\text {red }}=b_{\text {green }}=$ $0.0300 \mathrm{~m}, h_{\text {red }}=0.2100 \mathrm{~m}$, and $h_{\text {green }}=0.5500 \mathrm{~m}$ for the rectangles, and $b_{\text {triangle }}=0.0250 \mathrm{~m}$, and $h_{\text {triangle }}=0.2100 \mathrm{~m}$.

Table C.1 Standard CoM calculation table used in classic mechanics to solve CoM of a geometric shape creating a sledge hockey stick

\begin{tabular}{llllll}
\hline & $\mathbf{A}\left(\mathbf{m}^{\mathbf{2}}\right)$ & $\overline{\boldsymbol{x}}(\mathbf{m})$ & $\overline{\boldsymbol{y}}(\mathbf{m})$ & $\mathbf{A} \cdot \overline{\boldsymbol{x}}\left(\mathbf{m}^{\mathbf{3}}\right)$ & $\mathbf{A} \cdot \overline{\boldsymbol{y}}\left(\mathbf{m}^{\mathbf{3}}\right)$ \\
\hline \hline Triangle & 0.0026 & 0.0150 & 0.2750 & $3.9 \times 10^{-5}$ & $7.2 \times 10^{-4}$ \\
Rectangle (red) & 0.0063 & 0.0332 & 0.1050 & $2.1 \times 10^{-4}$ & $6.6 \times 10^{-4}$ \\
Rectangle (green) & 0.0165 & 0.0425 & 0.4150 & $7.0 \times 10^{-4}$ & $6.8 \times 10^{-3}$ \\
\hline \multicolumn{1}{c}{$\boldsymbol{\Sigma}$} & $\mathbf{0 . 0 2 5 4}$ & & & $\mathbf{9 . 5} \times \mathbf{1 0}^{-4}$ & $\mathbf{8 . 2 \times 1 0 ^ { - 3 }}$ \\
\hline
\end{tabular}

Then $\operatorname{st}\left(x_{i}, y_{i}\right)$ equals.

$$
\begin{aligned}
\overline{x_{0}} & =\left(\sum \mathrm{A} \bar{x}\right) / \mathrm{A} \\
& =0.0354 \mathrm{~m} \\
\overline{y_{0}} & =\left(\sum \mathrm{A} \bar{y}\right) / \mathrm{A} \\
& =0.3228 \mathrm{~m}
\end{aligned}
$$

Since the pick co-ordinates are centred within the width of the stick, and the 
local origin for $\operatorname{CoM}$ calculation is lateral to this location, a manipulation of

$$
\overline{x_{0}}-b / 2=0.0204 \mathrm{~m}
$$

should be used as the proper $\bar{x}$ distance from $P\left(x_{i}, y_{i}\right)$.

Table C.2 CoM inertia and radius of gyration used in classic mechanics for each geometric shape creating a sledge hockey sticks

\begin{tabular}{lll}
\hline & $I_{0}\left(\mathrm{kgm}^{2}\right)$ & $\rho_{0}(\mathrm{~m})$ \\
\hline \hline Triangle & $6.4 \times 10^{-6}$ & 0.0495 \\
Rectangle (red) & $2.3 \times 10^{-5}$ & 0.0606 \\
Rectangle (green) & $4.16 \times 10^{-4}$ & 0.0460 \\
\hline \hline \multicolumn{1}{c}{$\boldsymbol{\Sigma}$} & $\mathbf{4 . 5 \times 1 0 ^ { - 4 }}$ & $\mathbf{0 . 1 5 6 1}$ \\
\hline
\end{tabular}

A more intricate marker system is recommended for further human participant analysis. The top of the sledge was marked $(B T)$, and when upright $T 10$ and $C 7$ markers created a perpendicular $(\perp)$ line from $\mathbf{e}_{\mathbf{1}}$ at $B T$. As indicated in Chapter 4, participants created convex curved thoracic sections of the vertebrae; a vertebrae region-specific marker system is recommended for future research. Torso angle was segmented acquiring two calculated angles; lumbar spine $\angle T 10 B T \mathbf{e}_{1}$, and thoracic spine $\angle S^{\circ} T 10 \mathbf{e}_{1}$, for human participant computations of this model.

From Newton's first Law of Motion, Law of Inertia

$$
\sum F=0 \mathrm{~N}
$$

From Newton's second Law of Motion, Law of Acceleration

$$
\boldsymbol{F}=m \boldsymbol{a}
$$

From Newton's third Law of Motion, Law of Action and Reaction

$$
\boldsymbol{F}_{\boldsymbol{i}-\boldsymbol{j}}=-\boldsymbol{F}_{\boldsymbol{j}-\boldsymbol{i}}
$$

Let $F$ represent the sum of all force vectors under investigation

$$
\sum_{n}^{i} F=F_{i}+\cdots+F_{n}
$$




$$
\sum F=\left(F_{x}^{2}+F_{y}^{2}+F_{z}^{2}\right)^{1 / 2}
$$

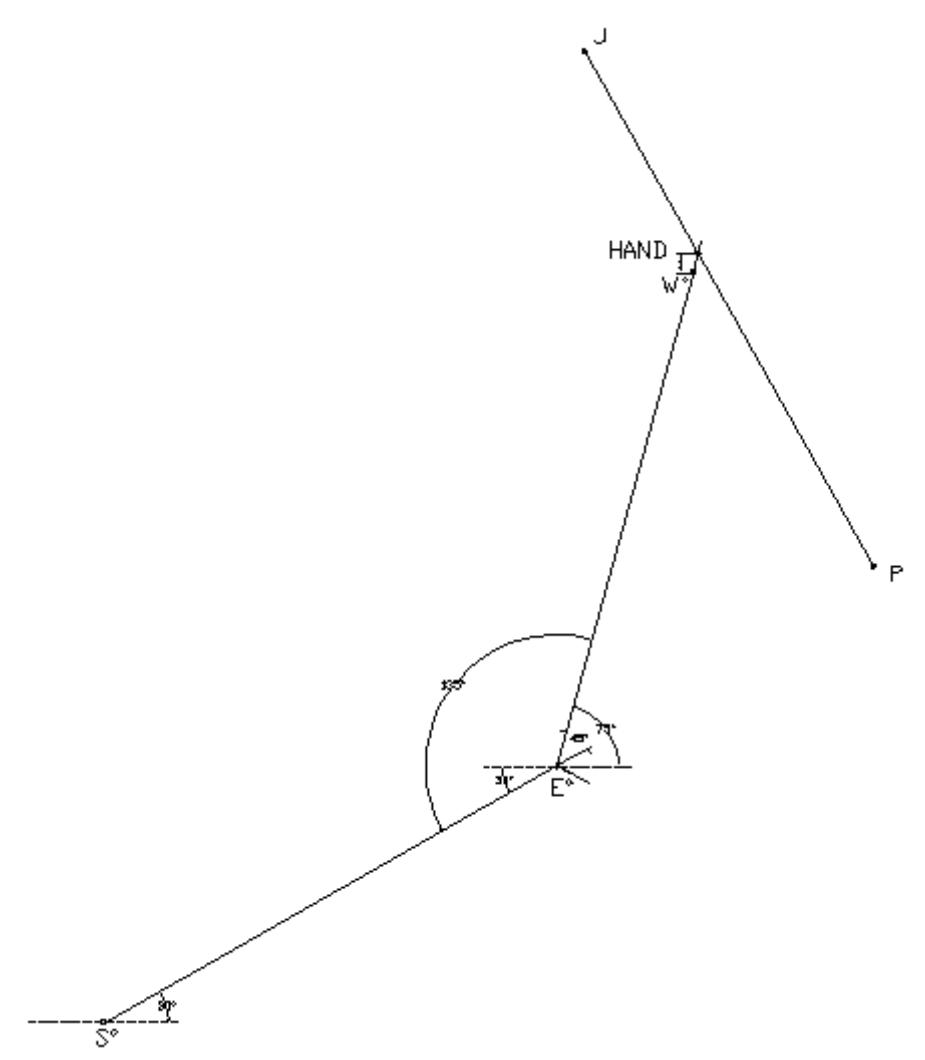

Figure C.7 Sagittal plane upper limb representation at start position. Palm-stick interface is represented at $H A N D$ assuming the $H A N D$ is along the vector created from the forearm.

From Winter, equations (5.1) and (5.2), equation (C.8) becomes,

$$
\begin{gathered}
R_{x p}-R_{x d}=m a_{x} \\
R_{y p}-R_{y d}-m g=m a_{y}
\end{gathered}
$$

respectively, where $R$ is the reaction forces at the proximal $(p)$ and distal (d) joints [96, p. 113]. 
As per classic mechanics, a relationship exists between position vectors $\boldsymbol{r}$ ( $r$ in metres), velocity $\boldsymbol{v}(v$ in metres per second), and acceleration $\boldsymbol{a}$ ( $\boldsymbol{a}$ in metres per second squared), where the terminal point $\boldsymbol{r}$ from the $O_{i}$ is dependent on time ( $t$ in seconds). From Tozeren, equations (2.7a), (2.8a), and $(2.8 \mathrm{~b})$ the relationship is derived [16, p. 40]:

$$
\boldsymbol{r}=\boldsymbol{r}(t)
$$

Substituting equation (C.1) into equation (C.14), we get,

$$
\boldsymbol{r}=x_{i} \mathbf{e}_{\mathbf{1}}+y_{i} \mathbf{e}_{2}
$$

implying equation (C.5) and equation (C.6) become

$$
\begin{aligned}
& x_{i}=x_{i}(t) \\
& y_{i}=y_{i}(t)
\end{aligned}
$$

respectively.

A relationship is then created by taking the derivative of equation (C.15) with respect to time.

$$
\begin{gathered}
\boldsymbol{v}=\frac{d \boldsymbol{r}}{d t} \\
=\frac{d x_{i}}{d t} \mathbf{e}_{1}+\frac{d y_{i}}{d t} \mathbf{e}_{2}
\end{gathered}
$$

The relation continues by taking the derivative of equation (C.19) with respect to time.

$$
\begin{gathered}
\boldsymbol{a}=\frac{d \boldsymbol{v}}{d t} \\
=\frac{d v_{x}}{d t} \mathbf{e}_{\mathbf{1}}+\frac{d v_{y}}{d t} \mathbf{e}_{2} \\
=\frac{d^{2} x_{i}}{d t^{2}} \mathbf{e}_{\mathbf{1}}+\frac{d^{2} y_{i}}{d t^{2}} \mathbf{e}_{2} \\
=\frac{d^{2} \boldsymbol{r}}{d t^{2}}
\end{gathered}
$$

where the result of the derivative of equation (C.20) is the double derivative of equation (C.14) as shown in equation (C.23). 
Taking this one-step further to eliminate potential errors when relating velocity with displacement (equation (C.19) represents velocity at a midpoint in time), Winters suggests to compute velocity and acceleration over $2 \cdot \Delta t$ [96]. From Winter, using the relations between equation (3.14) through to equation (3.17), and equation (3.18c), equation (C.19) and equation (C.21) become [96]:

$$
\begin{gathered}
v_{x_{i}}=\frac{x_{i+1}-x_{i-1}}{2 \Delta t} \\
a_{x_{i}}=\frac{x_{i+1}-2 x_{i}+x_{i-1}}{\Delta t^{2}}
\end{gathered}
$$

respectively.

Similarly, the angular relations are

$$
\begin{gathered}
\omega_{x_{i}}=\frac{\theta_{i+1}-\theta_{i-1}}{2 \Delta t} \\
\alpha_{x_{i}}=\frac{\theta_{i+1}-2 \theta_{i}+\theta_{i-1}}{\Delta t^{2}}
\end{gathered}
$$

where,

$$
\theta_{j i}=\tan ^{-1} \frac{y_{j}-y_{i}}{x_{j}-x_{i}}
$$

Proof is provided for $t_{0}$. The local origin for pick contact analysis $(0,0)$ occurs at $t_{\text {peak }}$, however, in order to prove the model is accurate, computations continue from the $G H J$. From this, the mathematical model is proven independent to MOCAP trajectory co-ordinates. Co-ordinate positions for $t_{0}$ are required for power analysis (Appendix C.2). The model is solved for $S L A M-80$ for simplicity.

\section{Sledge Hockey Skating Model Proof}

Let $S^{\circ}$ represent the $G H J$, and $P$ the pick of the stick.

Solve the model for $P_{0}$, where $S L A M-80$ elbow angle is $135^{\circ}$. 
Find $F_{S_{p e a k}^{\circ}}$ and $M_{S_{p e a k}^{\circ}}$

1. We know the sledge is $0.1000 \mathrm{~m}$ off the ground to where the gluteus region rests upon it. We know the torso angle from the hip is $\Lambda=40^{\circ}$, torso length is $0.9000 \mathrm{~m}$, sledge to hip joint-centre distance is $0.1300 \mathrm{~m}$, and hip to $G H J$ distance is $0.6200 \mathrm{~m}$.

Let $S L$ represent the bottom of the bucket aligned with hip joint-centre, where $\left(x_{\text {Hip }}, 0\right)$ is a local origin $(0,0)$. From equations (C.5) and (C.6), we get,

$$
\begin{aligned}
& x_{1}=0.0000 \mathrm{~m} \\
& y_{1}=0.1000 \mathrm{~m}
\end{aligned}
$$

Substitution into equation (C.1), we get,

$$
S L=0.0000 \mathbf{e}_{1}+0.1000 \mathbf{e}_{2}
$$

Let $H^{\circ}$ represent the hip at CoM. From equations (C.5) and (C.6), and $\Lambda$, we get,

$$
\begin{aligned}
& x_{2}=0.0996 \mathrm{~m} \\
& y_{2}=0.0836 \mathrm{~m}
\end{aligned}
$$

Substitution into equation (C.1), and adding to $\boldsymbol{S} \boldsymbol{L}$, we get,

$$
\boldsymbol{H}^{\circ}=0.0996 \mathbf{e}_{1}+0.1836 \mathbf{e}_{2}
$$

For the $G H J$, from equations (C.5) and (C.6), and $\Lambda$, we get,

$$
\begin{aligned}
& x_{3}=0.4750 \mathrm{~m} \\
& y_{3}=0.3985 \mathrm{~m}
\end{aligned}
$$

Substitution into equation (C.1), and adding to $\boldsymbol{H}^{\circ}$, we get,

$$
S^{\circ}=0.5746 \mathbf{e}_{1}+0.5821 \mathbf{e}_{2}
$$




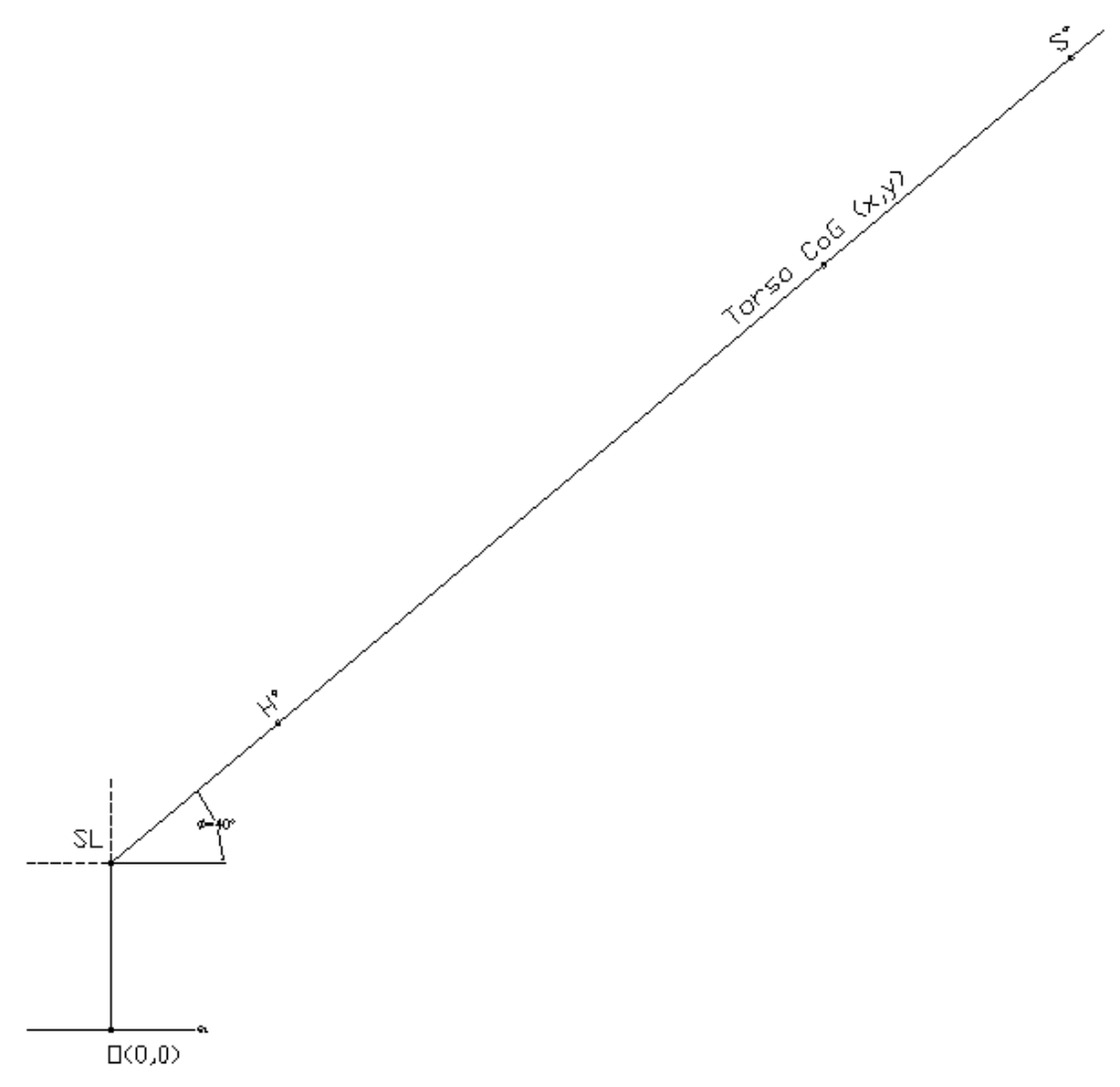

Figure C.8 Sagittal plane representation of the above-mentioned calculations creating the co-ordinates for the local origin $\left(S^{\circ}\right)$ for $S L A M-80$.

2. For $t_{0}$ :

We know upper arm $(U A)$ segment length is $0.4400 \mathrm{~m}, G H J$ to elbow is $0.3600 \mathrm{~m}$, upper arm CoM from proximal end is $0.436 U A$, and drop height $(\beta)$ is $+30^{\circ}$ to the horizon (parallel (II) to $G H J \mathbf{e}_{\mathbf{1}}$ ). $y$ direction is dependent upon drop height as the $G H J$ is the local origin for drop height measurements. We also know that joint to joint is centred within the segment leaving $0.0400 \mathrm{~m}$ extending past the joints for the upper arm.

Let $E^{\circ}$ represent the elbow, and $\beta \measuredangle \mathbf{e}_{1} S^{\circ} E^{\circ}$ (first or forth quadrant dependent upon start height), where $\mathbf{e}_{1} \cap S^{\circ}\left(x_{3}, y_{3}\right)$ creating a \| horizon. From equations (C.5) and (C.6), and $\beta$ we get, 


$$
\begin{aligned}
x_{4} & =0.3118 \mathrm{~m} \\
y_{4} & =0.1800 \mathrm{~m}
\end{aligned}
$$

Substitution into equation (C.1), and adding to $\boldsymbol{S}^{\circ}$, we get,

$$
\boldsymbol{E}^{\circ}=0.8864 \mathbf{e}_{1}+0.7621 \mathbf{e}_{2}
$$

Let $u a$ represent the upper arm CoM. From equations (C.5) and (C.6), the additional $0.0400 \mathrm{~m}$ at the end of the $G H J$, and $\beta$ we get,

$$
\begin{gathered}
u a=0.1918 \mathrm{~m} \\
d=u a-0.0400 \mathrm{~m} \\
x_{5}=0.1315 \mathrm{~m} \\
y_{5}=0.0759 \mathrm{~m}
\end{gathered}
$$

Substitution into equation (C.1), and adding to $S^{\circ}$, we get,

$$
\boldsymbol{u a}=0.7061 \mathbf{e}_{1}+0.6580 \mathbf{e}_{2}
$$

We know forearm $(F A)$ segment length is $0.4400 \mathrm{~m}$, elbow to wrist is $0.3500 \mathrm{~m}$, forearm CoM from proximal end is $0.430 \mathrm{FA}$, and elbow angle $(\psi)$ is $135^{\circ}$. We also know that joint to joint is centred within the segment leaving $0.0450 \mathrm{~m}$ extended past the joints for the forearm.

Let $W^{\circ}$ represent the wrist, $f a$ represent the forearm CoM, $\psi$ $\measuredangle u a E^{\circ} f a, o \quad \measuredangle f a E^{\circ} U A_{\text {distal }}$, and $\xi \measuredangle f a E^{\circ} \mathbf{e}_{\mathbf{1}}$ where $\mathbf{e}_{\mathbf{1}} \cap E^{\circ}\left(x_{4}, y_{4}\right)$ creating a || horizon. From the above known information, forearm angle $\mathrm{o}=45^{\circ}$.

$$
180^{\circ}=\psi+o
$$

By $\mathrm{Z}$ Rule $\beta_{0}$ dissects o creating a $\|$ horizon, then by Property of Opposite Angles we get,

$$
\xi=o \pm \beta
$$

understood as forearm angle from the horizon, $\left(\xi=75^{\circ}\right)$. 
From equations (C.5) and (C.6), and $\xi$, we get,

$$
\begin{aligned}
& x_{6}=0.0906 \mathrm{~m} \\
& y_{6}=0.3381 \mathrm{~m}
\end{aligned}
$$

Substitution into equation (C.1), and adding to $\boldsymbol{E}^{\circ}$, we get,

$$
\boldsymbol{W}^{\circ}=0.9770 \mathbf{e}_{1}+1.1002 \mathbf{e}_{2}
$$

From equations (C.5) and (C.6), the additional $0.0450 \mathrm{~m}$ at the end of the elbow, and $\xi$, we get,

$$
\begin{gathered}
f a=0.1892 \mathrm{~m} \\
d=f a-0.0450 \mathrm{~m} \\
x_{7}=0.0373 \mathrm{~m} \\
y_{7}=-0.1393 \mathrm{~m}
\end{gathered}
$$

Substitution into equation (C.1), and adding to $\boldsymbol{E}^{\circ}$, we get,

$$
f a=0.9238 \mathbf{e}_{1}+0.9014 \mathbf{e}_{2}
$$

We know wrist-stick internal angle is $45^{\circ}$. From human participants, internal wrist angle can be calculated from the above-mentioned method.

Let $\gamma$ represent wrist-stick internal angle, and $\chi \Varangle P W^{\circ} \mathbf{e}_{2}$, where $\mathbf{e}_{2} \cap$ $W^{\circ}\left(x_{6}, y_{6}\right)$ creating a $\|$ vertical line. Then

$$
\varepsilon=\gamma \pm \chi
$$

where the direction of $\chi$ is dependent upon $P\left(x_{9}, y_{9}\right)$. Then from $\xi$ and by properties for a triangle, we get,

$$
\varepsilon=90^{\circ}-\xi
$$

and

$$
\chi=30^{\circ}
$$

$x$ direction is dependent on pick location, and trigonometry is reversed as the angle of investigation is the third angle in the right angle triangle. 
From equations (C.5) and (C.6), $\chi,\left\|W^{\circ} P\right\|$, and Figure C.7, we get,

$$
\begin{gathered}
x_{9}=0.2000 \mathrm{~m} \\
y_{9}=-0.3464 \mathrm{~m}
\end{gathered}
$$

Substitution into equation (C.1), and adding to $\boldsymbol{W}^{\circ}$, we get,

$$
\boldsymbol{P}=1.1777 \mathbf{e}_{1}+0.7538 \mathbf{e}_{2}
$$

$s t$ is measured from the distal end, From equations (C.5) and (C.6), $\overline{x_{0}}$, and $\overline{y_{0}}$, we get

$$
\begin{gathered}
x_{10}=-0.0204 \mathrm{~m} \\
y_{10}=0.3228 \mathrm{~m}
\end{gathered}
$$

Substitution into equation (C.1), and adding to $\boldsymbol{P}^{\circ}$, we get,

$$
s t=1.1573 \mathbf{e}_{1}+1.0766 \mathbf{e}_{2}
$$

When pick $y=0.0000 \mathrm{~m}$, a reverse process can occur to find the coordinates of each of the above outlined points at $t_{\text {peak }}$. Since bones length is consistent, triangular relations can be made to solve for peak co-ordinates. As $S L A M-80$ theoretically has static joints at the elbow and wrist-stick, triangular relations can be made to solve for initial point of contact without any additional information. Figure C.10 and Figure C.11 outline the necessary triangular relations required for the reverse process. 


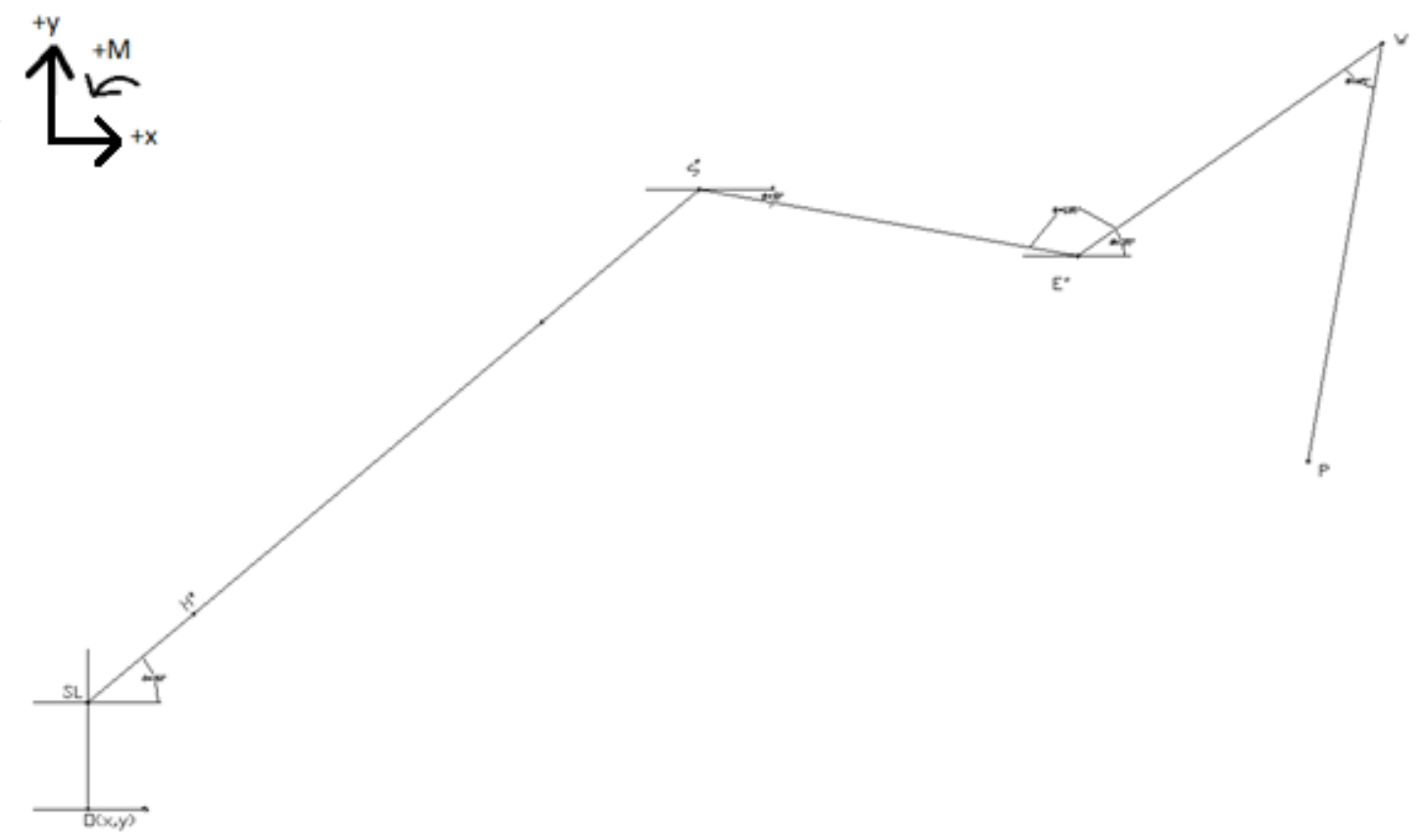

Figure C.9 A variation of the above-mentioned calculations for sagittal plane $S L A M-80$ analysis; drop height $-10^{\circ}$, elbow angle $135^{\circ}$, wrist-pick angle $45^{\circ}$.

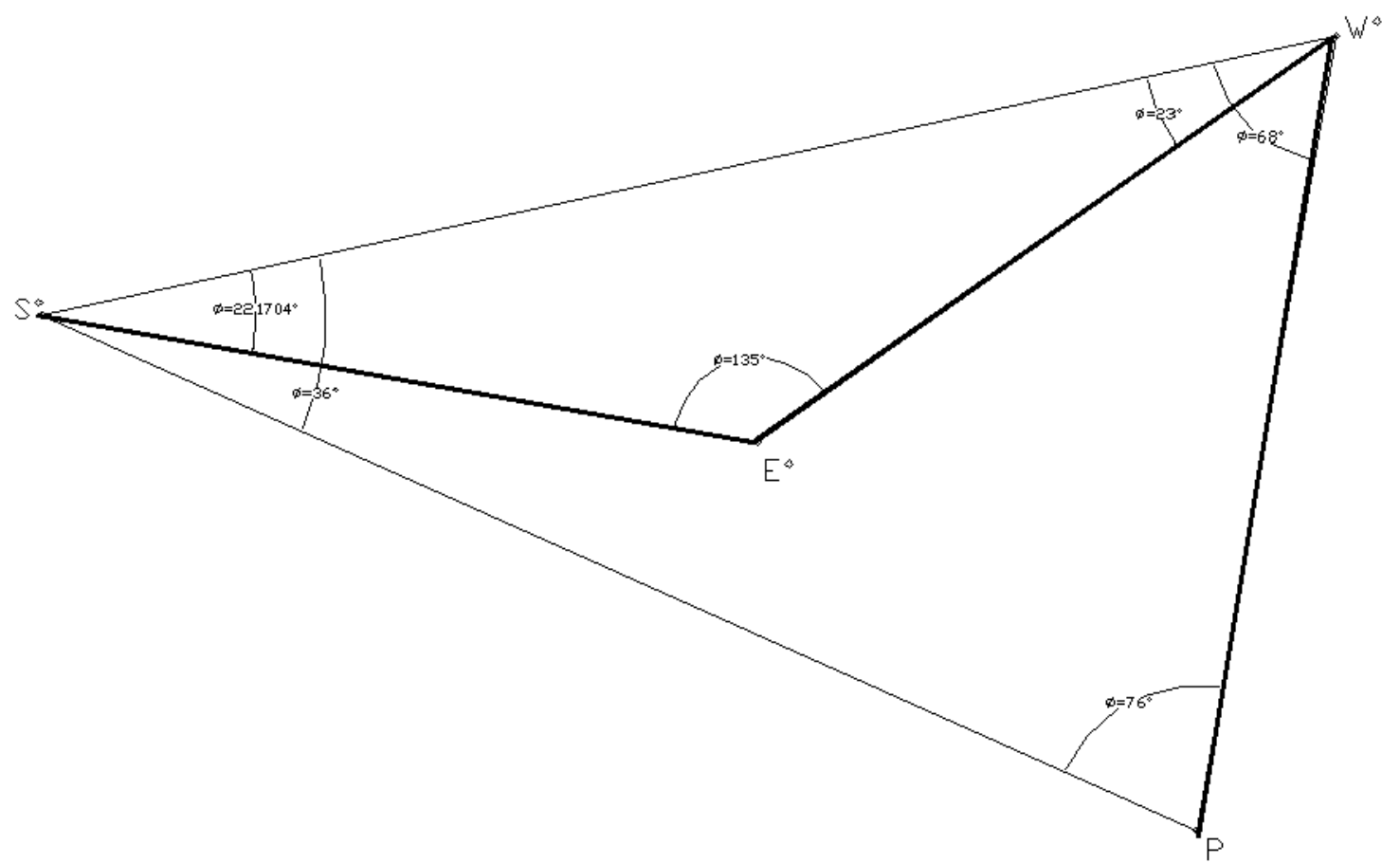

Figure C.10 Sagittal plane triangular relationships for $S L A M-80$ (thick black). Variations of combinations of outlined relationships are used to solve the system at pick $y=0.0000 \mathrm{~m}$. 


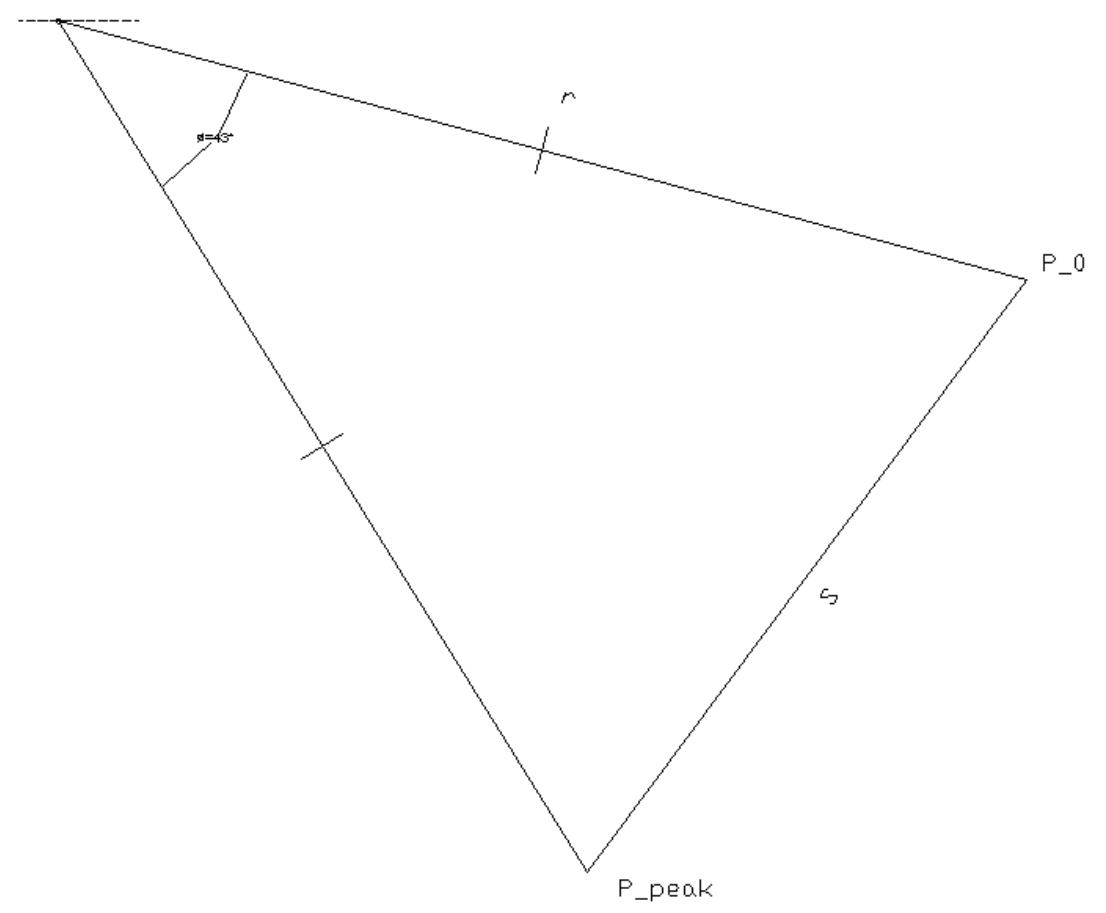

Figure C.11 Sagittal plane representation of the Pendulum Theory calculating $P_{0}$ to $P_{\text {peak }}$ for $S L A M-80$.

3. Let $m_{\text {equip }}$ equal the mass of the sticks and sledge in kilograms, and $g$ gravitational force. Let $m_{B W}=80.00 \mathrm{~kg}, m_{\text {equip }}=7.16 \mathrm{~kg}$, and $g=$ $9.81 \mathrm{~m} / \mathrm{s}$. Find $F_{\text {euip }}$ and $F_{B W}$ from equation (C.8),

$$
\begin{gathered}
F_{\text {euip }}=70.24 \approx 70 \mathrm{~N} \\
F_{B W}=784.80 \approx 785 \mathrm{~N}
\end{gathered}
$$

Pick-plant impact GRFs were determined from pick contact to point of recoil $\left(\sum_{t_{f}}^{t_{0}} R \neq 0 \mathrm{~N}\right)$ lasting a mean of $\Delta t_{I M P A C T}=0.0043 \mathrm{~s}$. From known trajectory co-ordinates, where $P(0,0)$, pick-ground angle $42^{\circ}$, wrist-stick angle $48^{\circ}$, elbow angle $124^{\circ}$, and resultant impact reaction force $R=1924 \mathrm{~N}$, the following inverse dynamic model is solved as per Winter [96, p. 86]: 
6. Find mass of segments from Winter Table 4.1 [96].

$$
\begin{gathered}
m_{U A}=0.028 B W \\
=2.24 \mathrm{~kg} \\
m_{F A}=0.022 B W \\
=1.28 \mathrm{~kg} \\
m_{S T K}=1.48 \mathrm{~kg}
\end{gathered}
$$

7. Find CoM of segments from Winter Table 4.1, equation (C.6), $\overline{x_{0}}, \overline{y_{0}}$, and Pythagorean Theorem (stick) [96, p. 86].

$$
\begin{gathered}
\operatorname{CoM}_{U A}=0.436 U A \\
=0.1918 \mathrm{~m} \\
\operatorname{CoM}_{\text {FAH }}=0.682 \mathrm{FA} \\
=0.3001 \mathrm{~m} \\
\text { CoM }_{\text {STK }}=0.3234 \mathrm{~m}
\end{gathered}
$$

8. Find segment radius of gyration from Winter Table 4.1 and equation (A.1) (stick) [96, p. 86].

$$
\begin{gathered}
\rho_{U A}=0.322 U A \\
=0.1417 \mathrm{~m} \\
\rho_{\text {FAH }}=0.682 \mathrm{FA} \\
=0.3001 \mathrm{~m} \\
\rho_{\text {STK }}=I_{S T K_{0}} / \mathrm{m}_{\text {STK }} \\
=0.0038 \mathrm{~m}
\end{gathered}
$$

9. Find segment inertia from equation (A.6).

$$
\begin{gathered}
I_{U A}=m_{U A} \rho_{U A} \\
=0.3174 \mathrm{kgm}^{2} \\
I_{F A}=m_{F A} \rho_{F A} \\
=0.5281 \mathrm{kgm}^{2} \\
I_{S T K}=m_{S T K} \rho_{S T K} \\
=0.0056 \mathrm{kgm}^{2}
\end{gathered}
$$

10. Find linear and angular velocity and acceleration from equation (C.24) through to equation (C.28) and MOCAP data for all segments.

$$
\begin{gathered}
v_{U A_{x}}=-0.1247 \mathrm{~m} / \mathrm{s} \\
v_{U A_{y}}=-0.7201 \mathrm{~m} / \mathrm{s} \\
a_{U A_{x}}=6.7969 \mathrm{~m} / \mathrm{s}^{2} \\
a_{U A_{y}}=-2.6808 \mathrm{~m} / \mathrm{s}^{2}
\end{gathered}
$$




$$
\begin{gathered}
\omega_{U A}=-4.4520 \mathrm{rad} / \mathrm{s} \\
\alpha_{U A}=46.4842 \mathrm{rad} / \mathrm{s}^{2} \\
v_{F A_{x}}=-1.0283 \mathrm{~m} / \mathrm{s} \\
v_{F A_{y}}=-1.5985 \mathrm{~m} / \mathrm{s} \\
a_{F A_{x}}=25.4316 \mathrm{~m} / \mathrm{s}^{2} \\
a_{F A_{y}}=78.6728 \mathrm{~m} / \mathrm{s}^{2} \\
\omega_{F A}=-1.1776 \mathrm{rad} / \mathrm{s} \\
\alpha_{F A}=335.2668 \mathrm{rad} / \mathrm{s}^{2} \\
v_{S T K_{x}}=-1.7432 \mathrm{~m} / \mathrm{s}^{2} \\
v_{S T K_{y}}=-0.8420 \mathrm{~m} / \mathrm{s}^{2} \\
a_{S T K_{x}}=99.1116 \mathrm{~m} / \mathrm{s}^{2} \\
a_{S T K_{y}}=50.2519 \mathrm{~m} / \mathrm{s}^{2} \\
\omega_{S T K}=-2.8478 \mathrm{rad} / \mathrm{s}^{2} \\
\alpha_{S T K}=285.4925 \mathrm{rad} / \mathrm{s}^{2}
\end{gathered}
$$

11. Find $F_{W^{\circ}}$ and $M_{W^{\circ}}$ from equation (C.11) through to (C.13), and equation (2.5), respectively (Figure C.12).

$$
\begin{gathered}
F_{W^{\circ}{ }_{x}}=1600 \mathrm{~N} \\
F_{W^{\circ}{ }^{\circ}}=1357 \mathrm{~N} \\
F_{W^{\circ}}=2098 \mathrm{~N} \\
M_{W^{\circ}}=-11 \mathrm{Nm}
\end{gathered}
$$

Therefore, the wrist is performing ulnar deviation. 


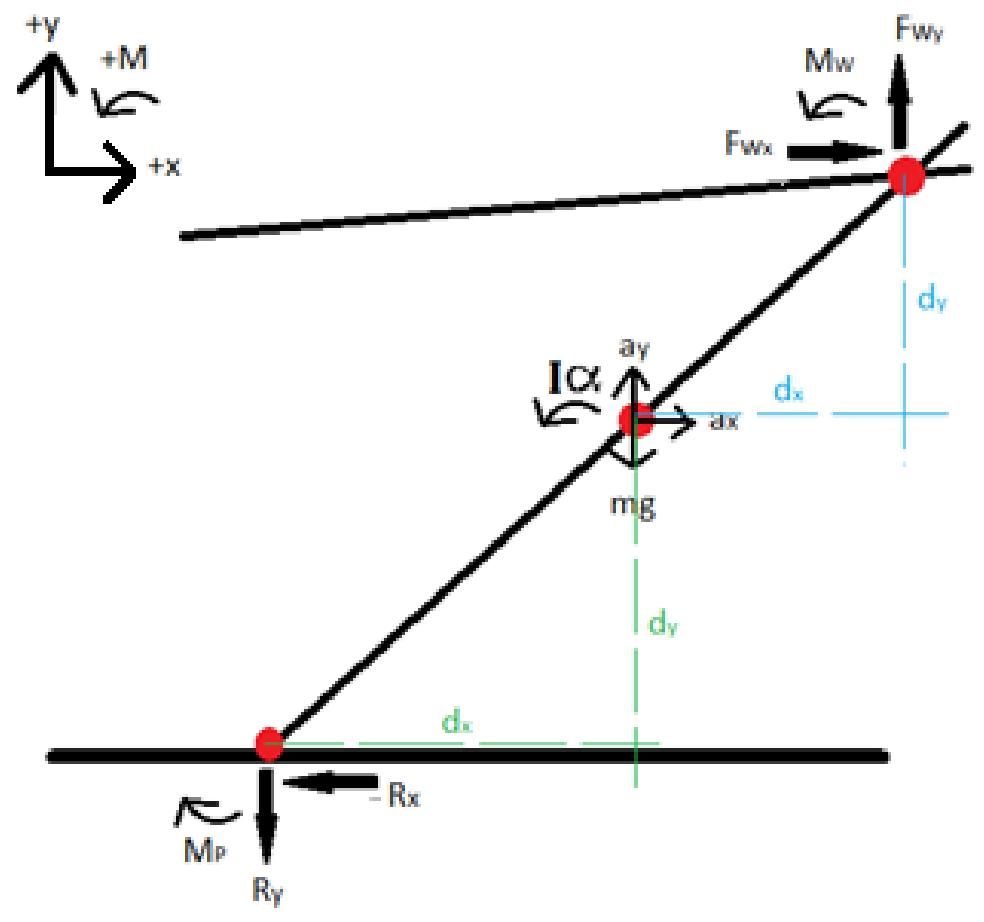

Figure C.12 Free body diagram $(F D B)$ at peak resultant reaction force solving for force and torque at the wrist. $P(0,0)$ and unknowns are assumed positive as per Winter [96].

12. Find $F_{E^{\circ}}$ and $M_{E^{\circ}}$ from equation (C.11) through to (C.13), and equation (2.5), respectively (Figure C.13).

$$
\begin{aligned}
F_{E^{\circ}{ }_{x}} & =1632 \mathrm{~N} \\
F_{E_{y}} & =1470 \mathrm{~N} \\
F_{E^{\circ}} & =2197 \mathrm{~N} \\
M_{E^{\circ}} & =616 \mathrm{Nm}
\end{aligned}
$$

Therefore, the elbow is flexing.

13. Find $F_{S^{\circ}}$ and $M_{S^{\circ}}$ from equation (C.11) through to (C.13), and equation (2.5), respectively (Figure C.14).

$$
\begin{gathered}
F_{S^{\circ}{ }_{x}}=1648 \mathrm{~N} \\
F_{S^{\circ}{ }_{y}}=1486 \mathrm{~N} \\
F_{S^{\circ}}=2219 \mathrm{~N} \\
M_{S^{\circ}}=1409 \mathrm{Nm}
\end{gathered}
$$

Therefore, the shoulder is undergoing flexion, and $291 \mathrm{~N}$ of in-vivo 
force is gained from impact $\left(F_{S^{\circ}}>R\right)$.
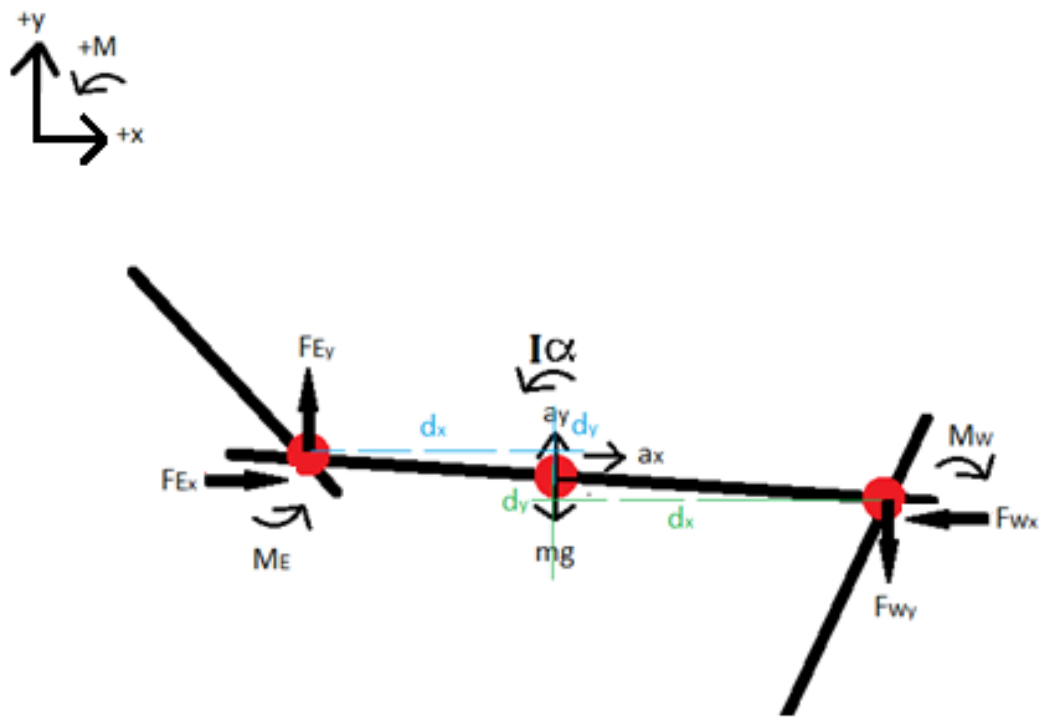

Figure C.13 FDB at peak resultant reaction force solving for force and torque at the elbow. $P(0,0)$ and unknowns are assumed positive as per Winter [96].

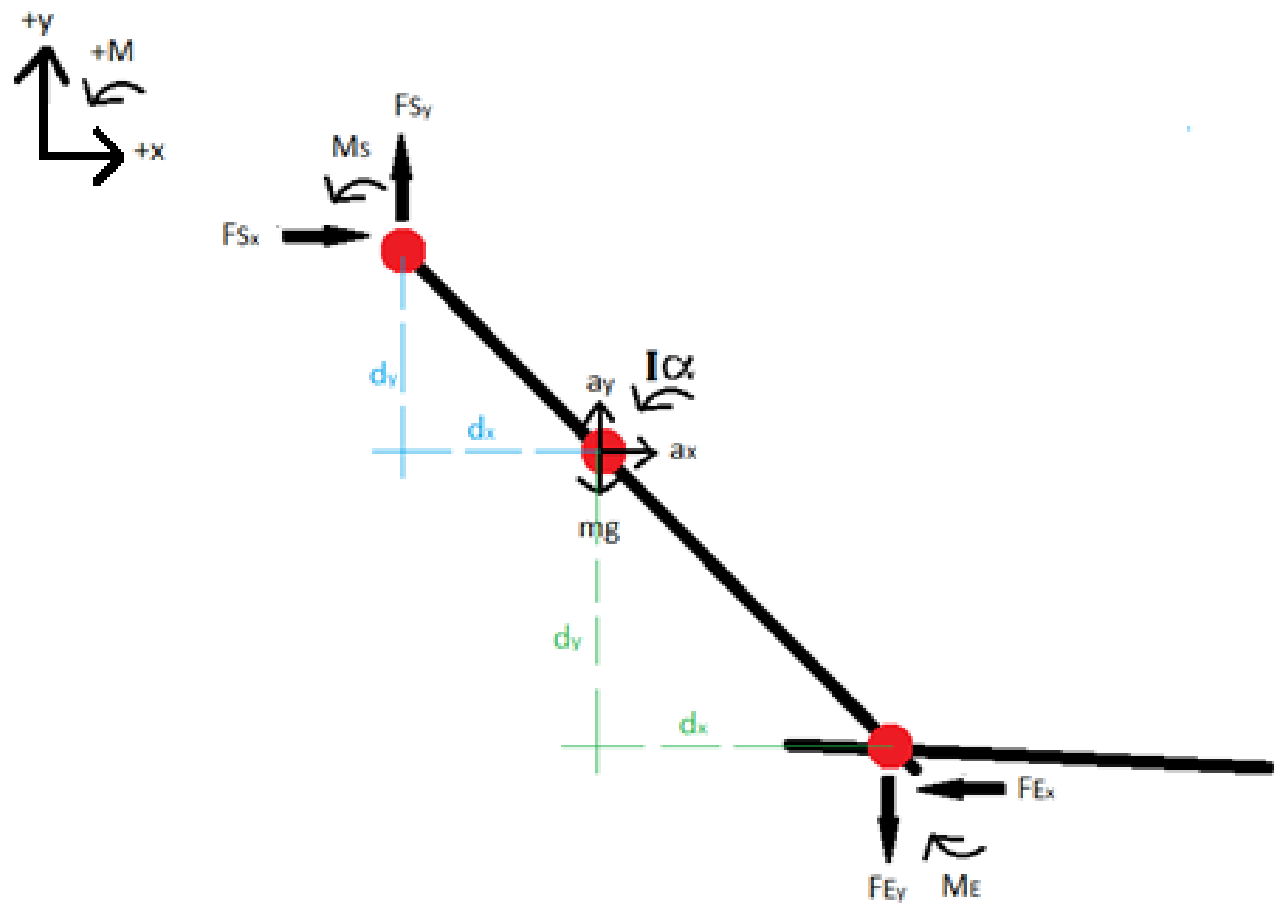

Figure C.14 FDB at peak resultant reaction force solving for force and torque at the shoulder. $P(0,0)$ and unknowns are assumed positive as per Winter [96]. 
Return to $\boldsymbol{S} \boldsymbol{L A M}-\mathbf{8 0}$ Applied Mathematical Model.

\section{C.2 Power for the $G H J$ produced from a downward pole in the sport of sledge hockey}

Limb segment power is determined by summing the power of each limb segment. Power enters on end of the segment and exits through to the adjoining segment, if it exists. For the downward poling upper limb segment, power must be summed for the upper arm, forearm, and sledge hockey stick (equation (C.29)) from equation (A.13). In order to get specific power, bodyweight is used to divide total limb segment power.

$$
\begin{aligned}
& P_{\text {Limb }}=\sum P_{U A}+P_{F A}+P_{S T K} \\
& P_{U A}=M \omega+F \cdot v \\
& =M_{S^{\circ}} \omega_{U A}+F_{E_{x}^{\circ}} v_{E_{x}^{\circ}}+F_{E_{y}^{\circ}} v_{E_{y}^{\circ}}+M_{E^{\circ}} \omega_{F A} \\
& P_{F A}=M \omega+F \cdot v
\end{aligned}
$$

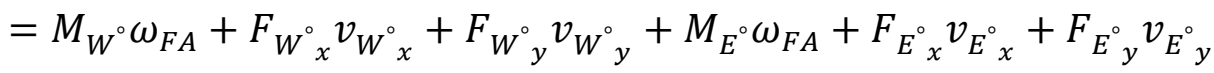

$$
\begin{aligned}
& P_{s t k}=M \omega+F \cdot v \\
& =M_{W^{\circ}} \omega_{A T K}+F_{W_{x}^{\circ}} v_{W_{x}^{\circ}}+F_{W^{\circ}{ }_{y}} v_{W^{\circ}{ }_{y}}
\end{aligned}
$$

Return to $\boldsymbol{S} \boldsymbol{L A M} \mathbf{M} \mathbf{8 0}$ Applied Mathematical Model. 


\section{Appendix D Research Ethics Approval and Requirements}

\section{D.1 Carleton University Research Ethics Board Approval}

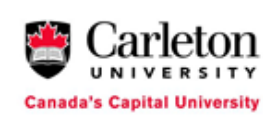

Ethics Clearance Form - Clearance Renewal

\author{
Carleton University \\ Research Ethics Office \\ Research Ethics Board \\ 511 Tory, 1125 Colonel By Drive \\ Ottawa, ON K1S 5B6 Canada \\ Tel: 613-520-2517, ethics@carleton.ca
}

This is to certify that the Carleton University Research Ethics Board has examined the application for ethical clearance. The REB found the research project to meet appropriate ethical standards as outlined in the Tri-Council Policy Statement: Ethical Conduct for Research Involving Human, 2nd edition, and the Carleton University Policies and Procedures for the Ethical Conduct of Research.

Original Date of Clearance: September 26, 2014

Renewal Date of Clearance: May 01, 2015

Researcher: Alicia Gal (Student Research: Master's Student)

Department: Faculty of Engineering and DesigniSystems and Computer Engineering (Department of)

University: Carleton University

Research Supervisor (if applicable): Adrian Chan and Dean Hay

Project Number: 102067

Alternate File Number (if applicable):

Project Title: Kinematic and kinetic analysis of the glenohumeral joint during a double poling motion. Funder (if applicable)

Clearance Expires: May 31, 2016

All researchers are governed by the following conditions:

Annual Status Report: You are required to submit an Annual Status Report to either renew clearance or close the file. Failure to submit the Annual Status Report will result in the immediate suspension of the project. Funded projects will have accounts suspended until the report is submitted and approved.

Changes to the project: Any changes to the project must be submitted to the Carleton University Research Ethics Board for approval. All changes must be approved prior to the continuance of the research.

Adverse events: Should a participant suffer adversely from their participation in the project you are required to report the matter to the Carleton University Research Ethics Board. You must submit a written record of the event and indicate what steps you have taken to resolve the situation.

Suspension or termination of clearance: Failure to conduct the research in accordance with the principles of the Tri-Council Policy Statement: Ethical Conduct for Research Involving Humans, 2nd edition and the Carleton University Policies and Procedures for the Ethical Conduct of Research may result in the suspension or termination of the research project.

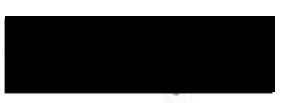

Louise Heslop

Chair, Carleton University Research Ethics Board

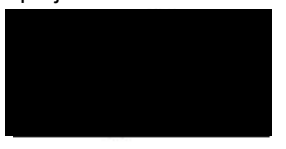

Andy Adler

Vice-Chair, Carleton University Research Ethics Board 


\section{D.2 University of Ottawa Health Sciences and Science Research Ethics Board}

\section{Approval}

File Number: H10-14-16

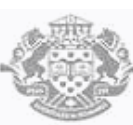

Date (mm/dd/yyyy): 10/13/2015

\section{Université d'Ottawa University of Ottawa \\ Bureau d'éthique et d'intégrité de la recherche Office of Research Ethics and Integrity}

\section{Ethics Approval Notice}

\section{Health Sciences and Science REB}

Principal Investigator / Supervisor / Co-investigator(s) / Student(s)

$\begin{array}{llll}\text { First Name } & \underline{\text { Last Name }} & \underline{\text { Affiliation }} & \underline{\text { Role }} \\ \text { Adrian } & \text { Chan } & \text { Engineering / Computer Engineering } & \text { Supervisor } \\ \text { Alicia } & \text { Gal } & \text { Others / Others } & \text { Student Researcher }\end{array}$

File Number: H10-14-16

Type of Project: Master's Thesis

Title: Kinematic and kinetic analysis of the glenohumeral joint during a double poling motion

Renewal Date (mm/dd/yyyy)

Expiry Date (mm/dd/yyyy)

Approval Type

$06 / 01 / 2015$

$05 / 31 / 2016$

Ia

(Ia: Approval, Ib: Approval for initial stage only)

Special Conditions / Comments:

N/A

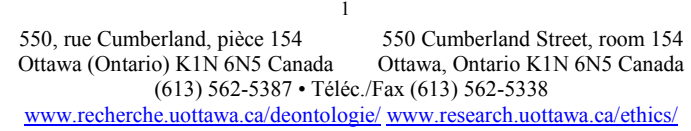




\section{D.3 Letter of Invitation}

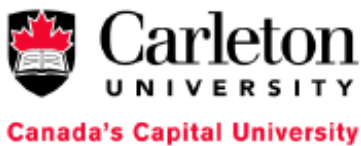

\section{Letter of Invitation}

Title: Kinematic and kinetic analysis of the glenohumeral joint during a double poling motion.

Date of ethics clearance: September 26, 2014

Ethics Clearance for the Collection of Data Expires: May 31, 2016

Dear Sir,

My name is Alicia Gal and I am a Master's student in the Ottawa-Carleton Institute for Biomedical Engineering at Carleton University. I am working on a research project under the supervision of Prof. Adrian Chan and Dr. Dean Hay.

I am writing to you today to invite you to participate in a study on the sport of sledge hockey. This study aims to provide 2 and 3-dimensional sport-specific descriptions of muscle movement during the linear stroking cycle.

This study involves one 120 minute session of sport-specific physical tasks through a motion capture system (10 infrared cameras and surface muscle activity recording electrodes) in the Human Kinetic Motion Laboratory - Lees Campus University of Ottawa in Ottawa, ON. This laboratory is $100 \%$ wheelchair accessible and arrangements can be made with ease. With your consent, sessions will be videorecorded and photographed.

While this project does involve some physical and emotional risks, care will be taken to protect your safety and identity. This will be done by following goldstandards for equipment application, as well as categorizing data. While no individual participant name will be revealed in the publication of results, it may be possible for the public to deduce some general and/or individual group information.

You will have the right to end your participation in the study at any time, for any reason. If you choose to withdraw, all the information you have provided will be removed from the study and destroyed.

No compensation will be provided. Personal data will be summarized and provide subsequently post completion of analysis.

All research data, including video-recordings and any notes will be password- 
protected and coded. Any hardcopies of data (including any handwritten notes or USB keys) will be kept in a locked cabinet at Carleton University. Research data will only be accessible by the researcher and the research supervisors.

This project was reviewed by the Carleton University Research Ethics Board, which provided clearance to carry out the research. Should you have questions or concerns related to your involvement in this research, please contact:

REB contact information:

Professor Andy Adler, Chair

Professor Louise Heslop, Vice-Chair

Research Ethics Board

Carleton University

1325 Dunton Tower

1125 Colonel By Drive

Ottawa, ON K1S 5B6

Tel: 613-520-2517

ethics@carleton.ca

If you would like to participate in this research project, or have any questions, please contact me at alicia.gal@carleton.ca

Sincerely,

Alicia Maria Gal 


\title{
D.4 Participant Procedure
}

\author{
Carleton \\ Participant Procedure \\ Note: bold and italic will be the procedure used for your study
}

5-20 healthy (absence of cardiovascular diseases, cancer, and metabolic disease), provincially ranked or higher male sledge hockey players aged between 18 and 45 years, who have been medically diagnosed with a physical impairment will be eligible for participation in this study. This impairment and its history will be documented for classification purposes only. A control group of 5-20 healthy able-bodied physically active (on average 3 days/week of elevating the heart rate above $70 \%$ of the estimated maximum heart rate as outlined by the Center for Disease Control and Prevention as vigorous-intensity), males 18 to 45 years, with no or limited knowledge of the physical tasks required may also be used. Control group members will portray a naive population to the linear stroking cycle in the sport of sledge hockey. A comparison of able-bodied naive movement will be made to physically-impaired trained movement. From this a more in depth understanding of physical limitations can be investigated. A pre-exercise screen meeting the Canadian Society of Exercise Physiology (CSEP) guidelines will be administered under the supervision of Alicia Maria Gal (BPHE \& FIS certified) to identify in the participant any risk factors or contraindications to exercise. Only low risk participants (i.e. absence of signs, symptoms, and major risk factors for cardiovascular disease, etc.) will be used. A physical activity readiness questionnaire (PAR-Q) will be signed and collected

All participants will be considered physically healthy and capable of potential participation in the components of the study. The tasks required of all participants are considered within the realm of their athletic ability. One session of multiple trials using the linear stroking pattern at the Human Kinetic Motion Laboratory - Lees Campus University of Ottawa in Ottawa, ON will be required. The laboratory is fully wheelchair accessible. Video recordings will be used for reference purposes. Photographs of instrument placement will be used for comparison and reference purpose. Consent for the publication of any images will be collected through informed-consent.

Baseline parameters will be set by analyzing the mechanics of a plywood arm designed to mimic the sport-specific motion.

Participant or Control Group: The entire experimental protocol is summarized as follows and should take an average of 2 hours with a maximum of 3 hours allotted per participant:

1) A pre-meeting will be conducted under the supervision of Alicia Maria Gal, to explain the risks, withdrawal procedures, benefits, and protocol of the proposed experiment. The PAR-Q and informed-consent forms will be completed and collected at this time. A brief description of the participant's functional impairment will be recorded for classification purposes only, if applicable. The remaining questions on the Interview Questionnaire will be asked at this time. All inquiries made on this questionnaire are 
categorization during analysis and publication (eg. male with right-side impairment stroking pattern/muscle activation/power production compared to male with no physical impairment). A question and answer period will be allotted to ensure total comprehension of the tasks being required. Anthropometric measurements will be collected for reconstruction of the skeleton via 3-D modelling computer software. No time limit is provided; to ensure all participants receive adequate time to raise questions and receive answers. However, average time to complete this session is 30 minutes.

2) Each participant will be required to wear non-reflective pants/shorts and shoes of their choice; and preform the trials bare-chested. Exposed skin minimizes the skinartefact effect of the marker system as well as eliminates signal-artefact created by the clothing.

A) Pre-session preparation will include skin preparation for surface electromyography (sEMG) electrode placement on the following bilateral muscles: latissimus dorsi and pectoralis major (trunk); triceps brachii and biceps brachii (upper arm); deltoid threesome (anterior, posterior, medial *if electrode is available) and trapezius (shoulders). Skin preparation will include shaving a small area of skin for each electrode (< 10 square $\mathrm{cm}$ ) with a sterilized, one-use razor, followed by a topical alcohol swab and light skin abrasion. This skin preparation is required to ensure good electrode-skin contact to reduced unwanted signal noise, and is a standard EMG recording protocol. EMG electrodes will be connected by cable to a portable EMG device that will be strapped to the lower torso of the participant with a device-specific harness (Delsys Inc.) or transmit wirelessly to a control box located on the floor at the base of the capture zone (BTS Corp.). Once the placement of EMG equipment has been completed, the participant will complete standard maximal voluntary isometric contractions (MVIC), sitting, for each of the muscle groups and used for baseline measures. Following MVICs, marker placement using a modified University of Ottawa Motion Analysis Model (UOMAM) for upper body marker placement will outline key components of the endoskeleton exteriorly on the skin; markers will be secured with two-sided adhesive tape. Once the placement of the marking system is complete, the participant will proceed to the capture area for calibration. Calibration preparation will include having the participant remain static secured in a sledge, on the origin force plate in the direction of travel, arms abducted holding a stick in each hand for a period no longer than 10 seconds. This is a baseline recording and will be used during analysis. Approximately 45-60 minutes will be required for pre-session preparation.

3) Encouragement will be provided to ensure an optimal environment for maximal performance.

B) The equipment used for indoor analysis is dependent upon the contact surface; artificial ice surface the participants will use their personal equipment; or rubber floor matting the participant will use a modified sledge and stick set designed for indoor 3-dimensional motion capture systems (non-reflective sledge with $64 \mathrm{~mm}$ rubber rollerblade wheels and stick set with reflective markings and padded picks). 
rubber rollerblade wheels and stick set with reflective markings and padded picks).

i) For each pattern the participants will complete up to 3 practice trials, to ensure precise force plate contact, followed by a minimum of 3 useable trials; a minimum of 2 minutes of rest will be allotted between trials: linear stroking pattern maximal linear pattern start-linear stroking pattern maximal start-linear stroking pattern.

The linear stroking pattern is defined as placing the sledge stationary outside the capture zone (at the marking indicated for precise force plate contact), on a random whistle the participant will propel themselves through the capture zone to the finish marker outside of the capture zone.

The start-linear stroking pattern is defined as placing the sledge stationary on the force places inside the capture zone (at the marking indicated for precise force plate contact), on a random whistle have the participant propel themselves through the remainder of the capture zone to the finish marker outside of the capture zone.

\section{Two methods will be used:}

1) with elbow brace at elbow angles 120, 135, 150 degrees start drop from above, at and below horizon

2) without elbow brace start drop from personal desired start position.

Approximately 30-45 minutes in total will be required for indoor analysis.
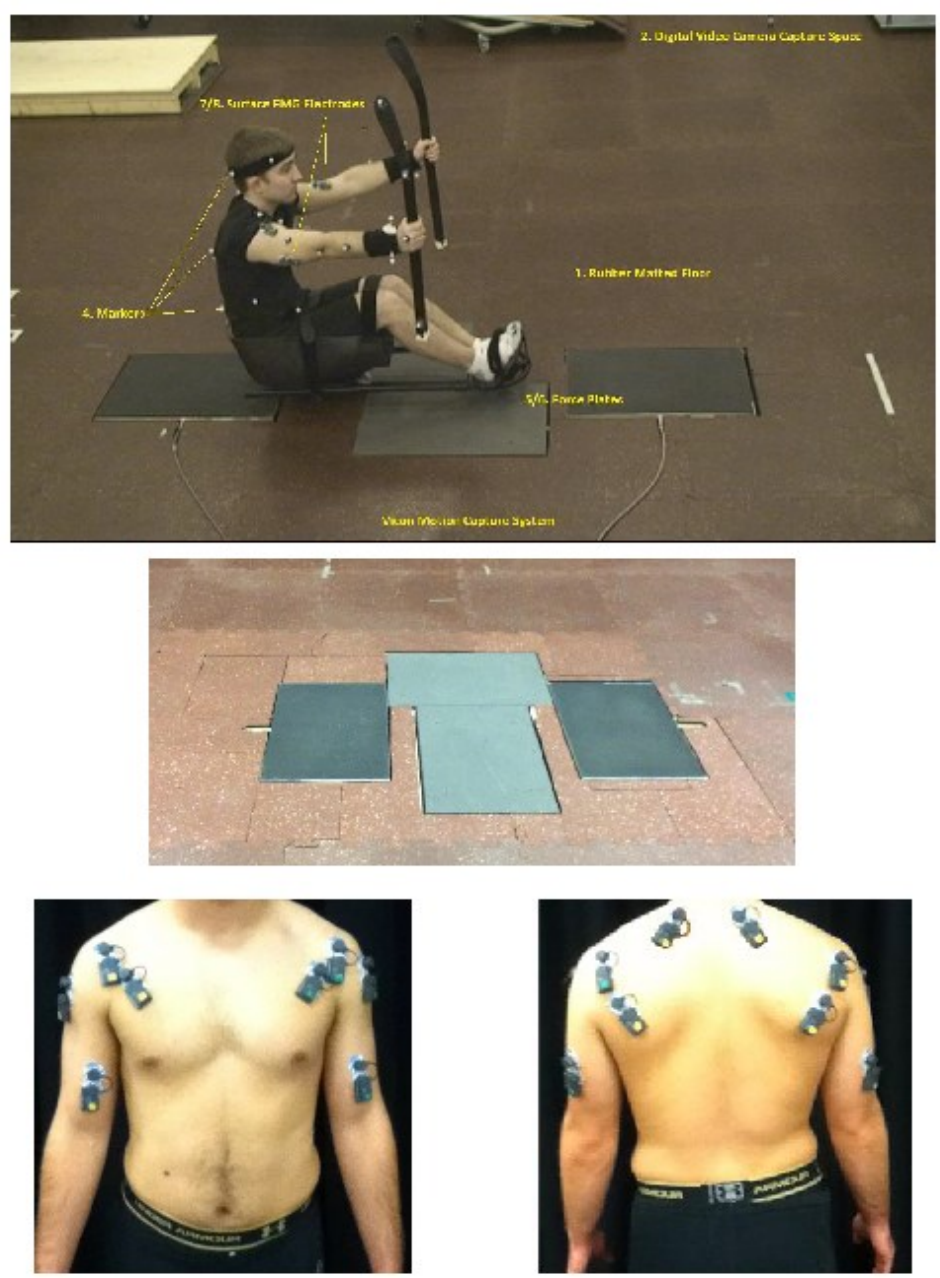


\section{D.5 Written Consent Form}

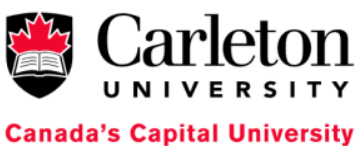

\section{Consent Form}

Title: Kinematic and kinetic analysis of the glenohumeral joint during a double poling motion.

Date of ethics clearance: September 26, 2014

Ethics Clearance for the Collection of Data Expires: May 31, 2016

I am invited to participate in a study on the sport of sledge hockey. This study aims to provide 2 and 3dimensional sport-specific descriptions of muscle movement during the linear stroking cycle. The researcher for this study is Alicia Gal in the OttawaCarleton Institute for Biomedical Engineering. She is working under the supervision of Dr. Adrian Chan in the Ottawa-Carleton Institute for Biomedical Engineering (Carleton University) and Dr. Dean Hay (Nipissing University).

This study involves one 120 minute session of sport-specific physical tasks through a motion capture system (10 infrared cameras with the application of a marker system onto your skin using two-sided tape and surface muscle activity recording electrodes) in the Human Kinetic Motion Laboratory - Lees Campus University of Ottawa in Ottawa, ON. This laboratory is $100 \%$ wheelchair accessible and arrangements can be made with ease.

With your consent, sessions will be video-recorded and photographed. Consent for video-recording and photography is mandatory to participate for reference purposes, however, consent for publication is not required. Possible publication of video-recordings and photographs can included but not limited to presentations, posters, conferences or scholarly publications.

This project will ask you about the history of physical impairment (if applicable) and your sledge hockey experiences for classification purposes. There are some potential physical and emotional risks; care will be taken to protect your safety and identity. This will be done by following gold-standards for equipment application, as well as categorizing data. While no individual participant name will be revealed in the publication of results, it may be possible for the public to deduce some general and/or individual group information. While these risks are expected to be minimal, we will take precautions to protect your safety and identity. Should you experience any distress at any time, you will be provided with

This document has been printed on both sides of a single sheet of paper. Page 1 of 3 Please retain a copy of this document for your records. 
the appropriate immediate treatment within the capabilities of the research team. If additional treatment is required, professionals will be sought out immediately to ensure your safety.

You have the right to end your participation in the study at any time, for any reason. You can withdraw by verbally stating or emailing the researcher or the research supervisor. If you withdraw from the study, all information will be removed from the study and destroyed. Furthermore, for those participants included in the study, current (and all future) exercise tests will be terminated immediately, and participants will be referred to a medical physician should they develop exerciseinduced symptoms such as chest discomfort, unexpected dyspnea, syncope, or abnormal electrocardiography events.

All research data, including video-recordings and any notes will be passwordprotected and coded. Any hardcopies of data (including any handwritten notes or USB keys) will be kept in a locked cabinet at Carleton University. Research data will only be accessible by the researcher and the research supervisors.

If you would like a copy of the finished research project, you are invited to contact the researcher to request an electronic copy which will be provided to you upon completion of analysis.

This project was reviewed by the Carleton University Research Ethics Board, which provided clearance to carry out the research. Should you have questions or concerns related to your involvement in this research, please contact:

REB contact information:

Professor Andy Adler, Chair

Professor Louise Heslop, Vice-Chair

Research Ethics Board

Carleton University

1325 Dunton Tower

1125 Colonel By Drive

Ottawa, ON K1S 5B6

Tel: 613-520-2517

ethics@carleton.ca

Researcher contact information: Alicia Gal

Systems and Computer Engineering

Carleton University

Tel: 613-402-9923

Email: alicai.gal@carleton.ca
Supervisor contact information: Dr. Adrian Chan

Systems and Computer Engineering

Carleton University

Tel: 6135202600 ext 1535

Email: adcchan@sce.carleton.ca

Page 2 of 3

This document has been printed on both sides of a single sheet of paper. Please retain a copy of this document for your records. 
Note: Some participants belong to publicly known team rosters

Any information obtained through this study that could lead to your direct identification will remain confidential and will be disclosed only with your permission. The data collected in this study will be kept strictly confidential. Your name will not appear in any report, publication, or presentation resulting from this study. However, it may be possible for the deduction of your identity due to categorization of the data in the final publishing.

Example: Elite Male 21 with left side Spina Bifida and 6 years experience in sledge hockey

or

Elite Male 19 able-bodied with no experience in sledge hockey (caption under picture)

Do you agree to be video-recorded and photographed for in-study purposes:

Yes _ No

Do you agree to be video-recorded and photographed for publication/presentation purposes:

Yes _ No

Have you been the recipient or have knowledge that internal repercussions have occurred from authority figures within your team atmosphere due to the participation or lack-thereof from this study:

Yes No

Are you on average physically active 3 times / week with a heart rate above $70 \%$ your calculated maximum heart rate?<smiles>C=[AsH2]CC</smiles>

Would you like a copy of your personal results upon completion: Yes No

Signature of participant

Date

Signature of researcher

Date Page 3 of 3

This document has been printed on both sides of a single sheet of paper. Please retain a copy of this document for your records. 


\section{D.6 Physical Activity Readiness Questionnaire $(P A R-Q)[111]$ \\ Physical Activity Readiness Questionnaire - PAR-Q \\ (revised 2002) \\ PAR-Q \& YOU}

(A Questionnaire for People Aged 15 to 69)

Regular physical activity is fun and healthy, and increasingly more people are starting to become more active every day. Being more active is very safe for most people. However, some people should check with their doctor before they start becoming much more physically active.

If you are planning to become much more physically active than you are now, start by answering the seven questions in the box below. If you are between the ages of 15 and 69 , the PAR-Q will tell you if you should check with your doctor before you start. If you are over 69 years of age, and you are not used to being very active, check with your doctor.

Common sense is your best guide when you answer these questions. Please read the questions carefully and answer each one honestly: check YES or NO.

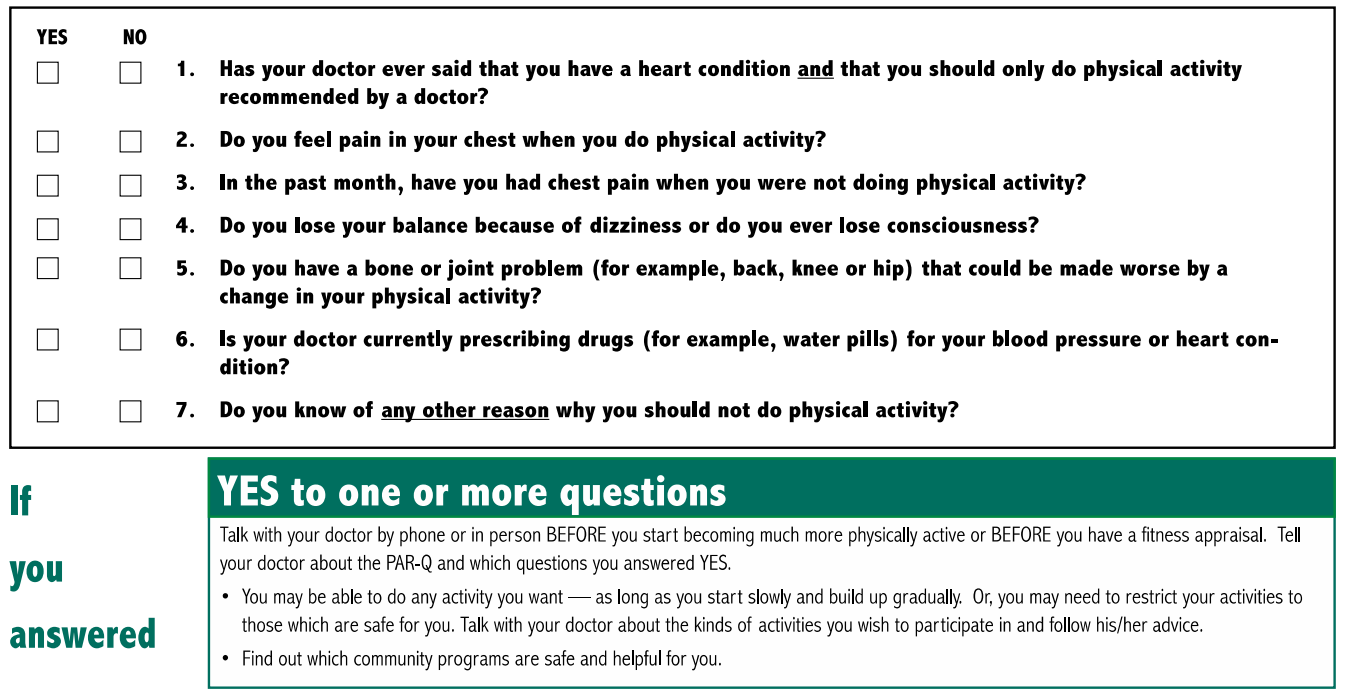

\section{NO to all questions}

If you answered NO honestly to all PAR-Q questions, you can be reasonably sure that you can:

- start becoming much more physically active - begin slowly and build up gradually. This is the safest and easiest way to go.

- take part in a fitness appraisal - this is an excellent way to determine your basic fitness so that you can plan the best way for you to live actively. It is also highly recommended that you have your blood pressure evaluated. If your reading is over $144 / 94$, talk with your doctor before you start becoming much more physically active.

DELAY BECOMING MUCH MORE ACTIVE:

- if you are not feeling well because of a temporary illness such as a cold or a fever - wait until you feel better; or

- if you are or may be pregnant - talk to your doctor before you start becoming more active.

PLEASE NOTE: If your health changes so that you then answer YES to any of the above questions, tell your fitness or health professional. Ask whether you should change your physical activity plan

Informed Use of the PAR-Q: The Canadian Society for Exercise Physiology, Health Canada, and their agents assume no liability for persons who undertake physical activity, and if in doubt after completing this questionnaire, consult your doctor prior to physical activity

No changes permitted. You are encouraged to photocopy the PAR-Q but only if you use the entire form.

NOTE: If the PAR-Q is being given to a person before he or she participates in a physical activity program or a fitness appraisal, this section may be used for legal or administrative purposes

"I have read, understood and completed this questionnaire. Any questions I had were answered to my full satisfaction."

NAME

SIGNATURE

SIGNATURE OF PARENT

or GUARDIAN (for participants under the age of majority)

DATE

WITNESS

Note: This physical activity clearance is valid for a maximum of 12 months from the date it is completed and becomes invalid if your condition changes so that you would answer YES to any of the seven questions. 


\section{D.7 Questionnaire Consent Form}

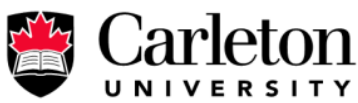

Canada's Capital University

\section{Questionnaire Consent Form}

Title: Kinematic and kinetic analysis of the glenohumeral joint during a double poling motion.

Date of ethics clearance: September 26, 2014

Ethics Clearance for the Collection of Data Expires: May 31, 2016

This is a study on the sport of sledge hockey. This study aims to provide 2 and 3dimensional sport-specific descriptions of muscle movement during the linear stroking cycle. The researcher for this study is Alicia Gal in the OttawaCarleton Institute for Biomedical Engineering. She is working under the supervision of Dr. Adrian Chan in the Ottawa-Carleton Institute for Biomedical Engineering (Carleton University) and Dr. Dean Hay (Nipissing University).

This study involves one 120 minute session of sport-specific physical tasks through a motion capture system (10 infrared cameras and surface muscle activity recording electrodes) in the Human Kinetic Motion Laboratory - Lees Campus University of Ottawa in Ottawa, ON. This laboratory is $100 \%$ wheelchair accessible and arrangements can be made with ease. With your consent, sessions will be video-recorded and photographed

While this questionnaire does involve some emotional risks, you have the right to refuse to answer any of the questions. Should you feel some distress, you are encouraged to speak to the researcher who will direct you to support services. This questionnaire will ask you about the history of physical impairment and your sledge hockey experiences for classification purposes.

You have the right to end your participation in the study at any time, for any reason. You can withdraw by verbally stating or emailing the researcher or the research supervisor. If you withdraw from the study, all information will remain the property of the principle researcher Alicia Maria Gal.

All research data, including video-recordings and any notes will be passwordprotected and coded. Any hardcopies of data (including any handwritten notes or USB keys) will be kept in a locked cabinet at Carleton University. Research data will only be accessible by the researcher and the research supervisors.

This project was reviewed by the Carleton University Research Ethics Board, which provided clearance to carry out the research. Should you have questions or 
concerns related to your involvement in this research, please contact:

REB contact information:

Professor Andy Adler, Chair

Professor Louise Heslop, Vice-Chair

Research Ethics Board

Carleton University

1325 Dunton Tower

1125 Colonel By Drive

Ottawa, ON K1S 5B6

Tel: 613-520-2517

ethics@carleton.ca

Researcher contact information:

Alicia Gal

Systems and Computer Engineering

Carleton University

Tel: 613-402-9923

Email: alicai.gal@carleton.ca
Supervisor contact information:

Dr. Adrian Chan

Systems and Computer Engineering

Carleton University

Tel: 6135202600 ext 1535

Email: adcchan@sce.carleton.ca 


\section{D.8 Interview Questionnaire}
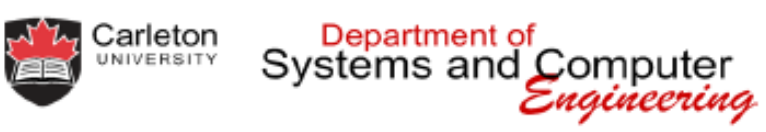

ID \#:

\section{Interview Questions}

Physical Impairment:
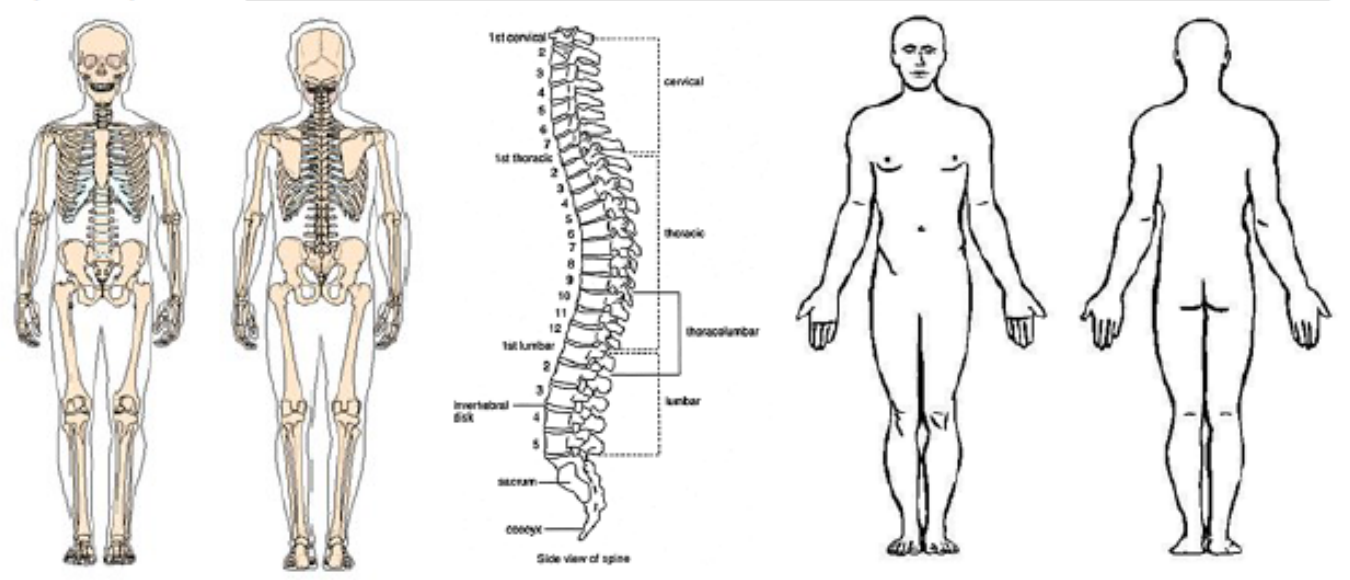

History:

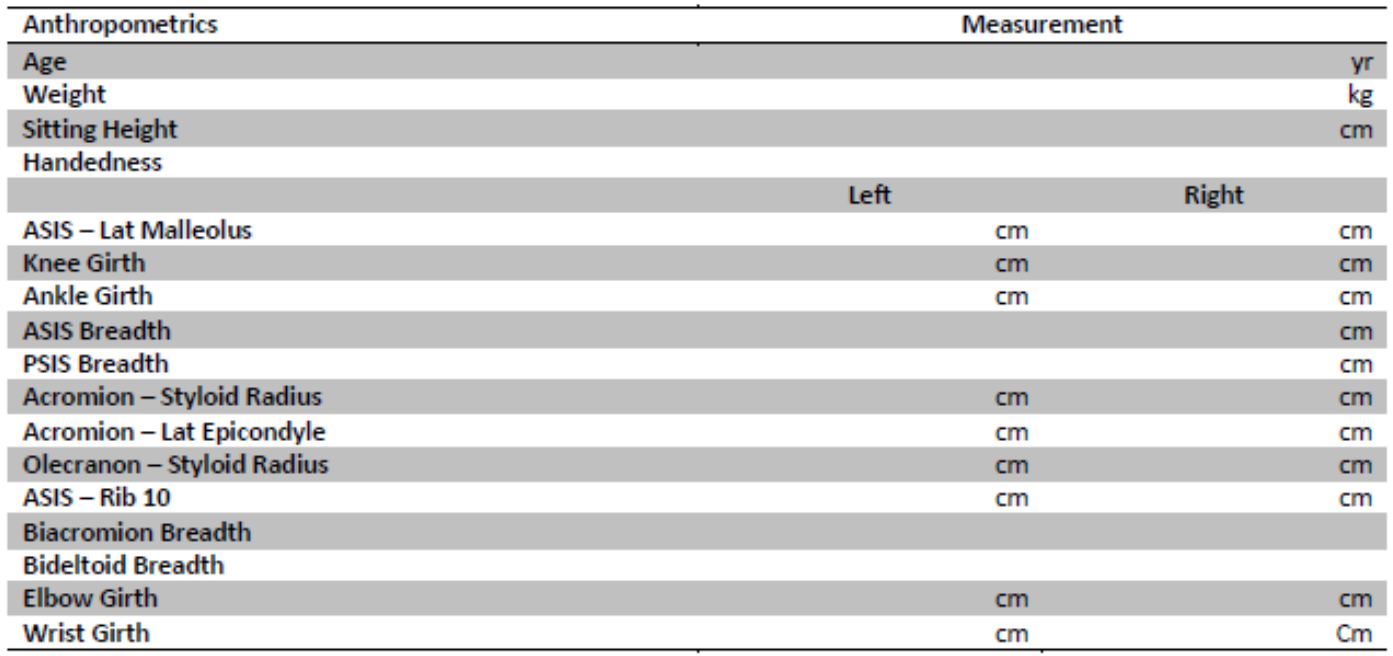


Sledge Hockev Historv:

a) Years Played Recreational:

b) Years Played Elite:

c) Position:

d) Previous Experience with Hockey:

\begin{tabular}{r|rrr}
\hline Stick Marker Placement & Pick $(\mathrm{cm})$ & Joint $(\mathrm{cm})$ & Blade $(\mathrm{cm})$ \\
\hline Left & & \\
Right & &
\end{tabular}

Muscle MVIC

Left (kg)

Right (kg)

Biceps Brachii

Triceps Brachii

Anterior Deltoid

Posterior Deltoid

Medial Deltoid

Trapezius

Pectoralis Major

Latissimus Dorsi

$70 \%$ Maximum Heart Rate $=220-$

$=$

$(0.7)=$

$220-$ age $=\operatorname{xxx}(0.7)=$

Notes:

\section{(kg)}

Return to Research Ethic Boards Approval and Requirements. 


\section{Appendix E Amendments}

\section{E.1 Interview Questionnaire}
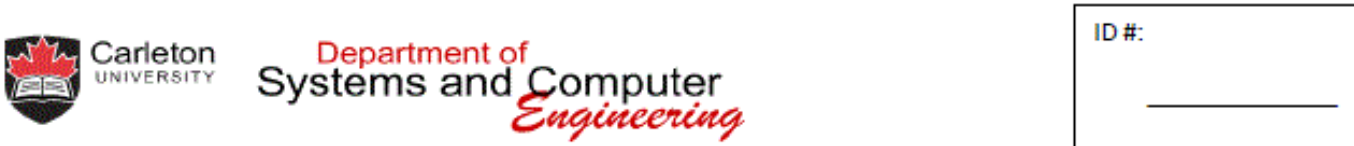

\section{Interview Questions}

Physical Impairment:
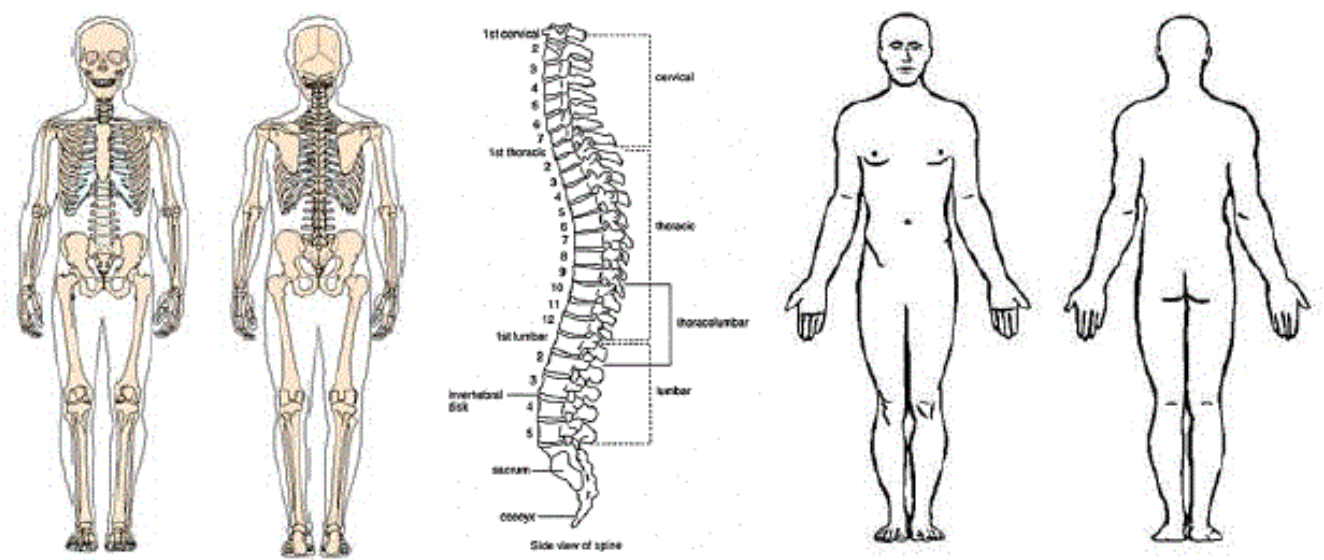

History:

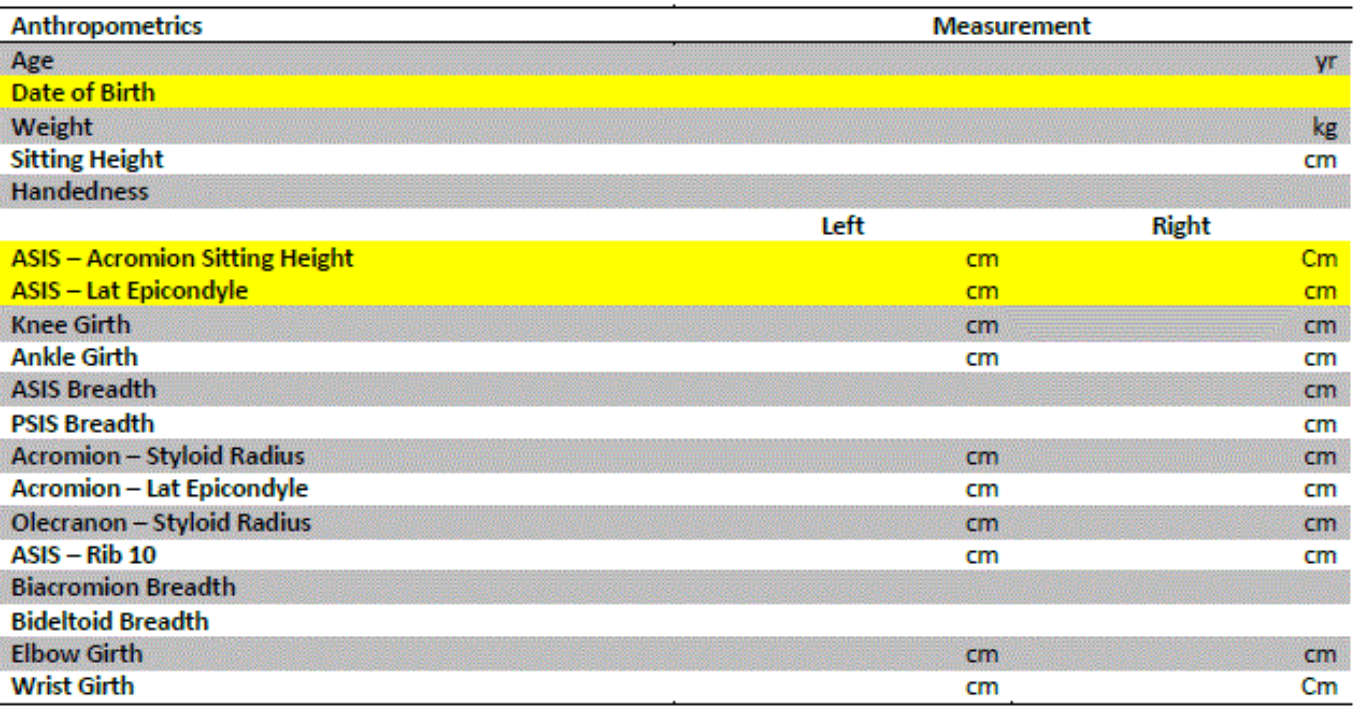


Sledge Hockey History:

a) Years Played Recreational:

b) Years Played Elite:

c) Position:

d) Previous Experience with Hockey:

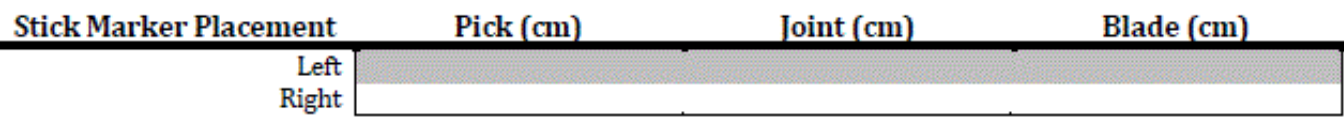

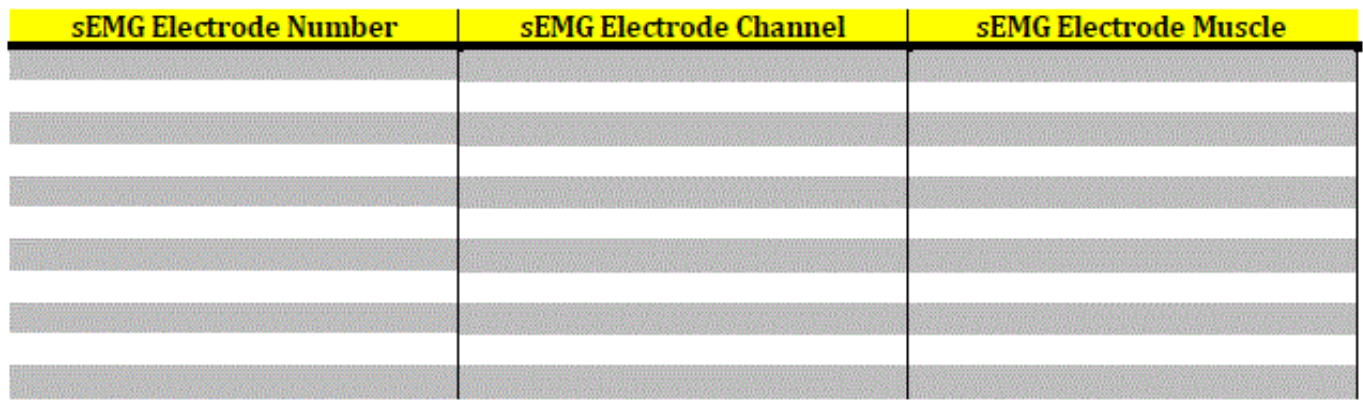

Notes: 


\section{References}

[1] J. Billette and T. Janz, "Injuries in Canada: Insights from the Canadian community health survey," Statistic Canada, 27-Nov-2015. [Online]. Available: http://www.statcan.gc.ca/pub/82-624-x/2011001/article/11506-eng.htm. [Accessed: 11-Nov-2016].

[2] National Defence and the Canadian Armed Forces, "Canadian Forces' casualty statistics (Afghanistan)," National Defence and the Canadian Armed Forces, 10Jun-2013. [Online]. Available: http://www.forces.gc.ca/en/news/article.page?doc=canadian-forces-casualtystatistics-afghanistan/hie8w9c9. [Accessed: 12-Nov-2016].

[3] D. F. Apple Jr, R. Cody, and A. Allen, "Overuse syndrome of the upper limb in people with spinal cord injury," Strain, vol. 24, p. 48, 1996.

[4] S. B. Kinsella and R. L. Carl, "Upper extremity overuse injuries," Clin. Pediatr. Emerg. Med., vol. 14, no. 4, pp. 318-326, 2013.

[5] R. Arvin Zifchock, I. Davis, J. Higginson, S. McCaw, and T. Royer, "Side to side differences in overuse running injury susceptibility: A retrospective study," Hum. Mov. Sci., vol. 27, pp. 888-902, 2008.

[6] A. Hreljac, R. N. Marshall, and P. A. Hume, "Evaluation of lower extremity overuse injury potential in runners:," Med. Sci. Sports Exerc., pp. 1635-1641, Sep. 2000.

[7] T. G. Sanders, M. Zlatkin, and J. Montgomery, "Imaging of glenohumeral instability," Semin. Roentgenol., vol. 45, no. 3, pp. 160-179, Jul. 2010.

[8] Hockey Canada, "Sledge hockey coaching resource." 2009.

[9] International Paralympic Committee, "IPC ice sledge hockey," 2015. [Online]. Available: https://www.paralympic.org/ice-sledge-hockey.

[10] Hockey Canada, "Sledge hockey facts," National Sledge Team, 2016. [Online]. Available: http://www.hockeycanada.ca/en-ca/TeamCanada/Men/Sledge/History/Sledge-Hockey-Facts. [Accessed: 11-Nov-2016].

[11] K. Lomond and R. Wiseman, "Sledge hockey mechanics take toll on shoulder: Analysis of propulsion technique can help experts design training programs to prevent injury," Biomechanics, p. 71, Aug-2003.

[12] Ivanaivanova, Russian national sledge hockey defeated the US team at the Paralympic Games in Sochi. 2014.

[13] H. E. J. Veeger and F. C. T. van der Helm, "Shoulder function: The perfect compromise between mobility and stability," J. Biomech., vol. 40, no. 10, pp. 2119 2129, Jan. 2007.

[14] A. M. Hill et al., "The clinical assessment and classification of shoulder instability," Curr. Orthop., vol. 22, no. 3, pp. 208-225, Jun. 2008.

[15] C. R. Ethier and C. A. Simmons, Introductory biomechanics: From cells to organisms. Cambridge; New York: Cambridge University Press, 2007.

[16] A. Tözeren, Human body dynamics: Classical mechanics and human movement. New York: Springer, 2000.

[17] R. C. Hibbeler, Engineering mechanics statics \& dynamics, 13th ed. New Jersey: Prentice Hall, 2012.

[18] N. Webborn, S. Willick, and J. C. Reeser, "Injuries among disabled athletes during the 2002 Winter Paralympic Games," Med. Sci. Sports Exerc., vol. 38, no. 5, pp. 
811-815, May 2006.

[19] J. Hawkeswood, H. Finlayson, R. O'Connor, and H. Anton, “A pilot survey on injury and safety concerns in international sledge hockey," Int. J. Sports Phys. Ther., vol. 6, no. 3, p. 173, 2011.

[20] J. Hotzman et al., "Measurer's handbook: US Army and Marine Corps anthropometric surveys, 2010-2011," DTIC Document, 2011.

[21] C. C. Gordon et al., "2012 snthropometric survey of US Army personnel: Methods and summary statistics," DTIC Document, 2014.

[22] A. R. Tilley, The measure of man and woman: Human factors in design, Revised. New York: Wiley, 2002.

[23] A. M. Gal, D. C. Hay, and A. D. C. Chan, "2 and 3-dimensional biomechanical analysis of the linear stroking cycle in the sport of sledge hockey (Glenohumeral joint kinematic, kinetic and surface EMG muscle modeling on and off ice)," in 13th International Symposium on 3D Analysis of Human Movement, Lausanne, Swizerland, 2014, pp. 108-111.

[24] L. Gastaldi, S. Pastorelli, and S. Frassinelli, "A biomechanical approach to paralympic cross-country sit-ski racing," Clin. J. Sport Med., vol. 22, no. 1, pp. 5864, 2012.

[25] E. Langelier, S. Martel, A. Millot, J.-L. Lessard, C. Smeesters, and D. Rancourt, "A sit-ski design aimed at controlling centre of mass and inertia," J. Sports Sci., vol. 31, no. 10, pp. 1064-1073, Jun. 2013.

[26] G. Giarmatzis, I. Jonkers, M. Wesseling, S. Van Rossom, and S. Verschueren, "Loading of hip measured by hip contact forces at different speeds of walking and running: Hip loading measured by HCFS at different speeds of walking and runnig," J. Bone Miner. Res., vol. 30, no. 8, pp. 1431-1440, Aug. 2015.

[27] G. Bergmann, "Hip 98: Loading of the hip joint." Biomechanics Lab., Free University of Berlin, 2001.

[28] A. Vinther et al., "Slide-based ergometer rowing: Effects on force production and neuromuscular activity: Force and EMG in slide-based ergometer rowing," Scand. J. Med. Sci. Sports, p. n/a-n/a, Feb. 2012.

[29] A. M. Koontz, R. A. Cooper, M. L. Boninger, Y. Yang, B. G. Impink, and L. H. V. van der Woude, "A kinetic analysis of manual wheelchair propulsion during start-up on select indoor and outdoor surfaces," J. Rehabil. Res. Dev., vol. 42, no. 4, p. 447, 2005.

[30] R. N. Robertson, M. L. Boninger, R. A. Cooper, and S. D. Shimada, "Pushrim forces and joint kinetics during wheelchair propulsion," Arch. Phys. Med. Rehabil., vol. 77, no. 9, pp. 856-864, 1996.

[31] T. R. Ebert, D. T. Martin, B. Stephens, and R. T. Withers, "Power output during a professional men's road-cycling tour," Int. J. Sports Physiol. Perform., vol. 1, no. 4, p. 324, 2006.

[32] A. M. Gal, A. D. C. Chan, and D. C. Hay, "Validating a Solid-Static Single-Armed Male Prototype Tasked to Produce Dynamic Movement from the Shoulder Through the Preparation Phase," in World Congress on Medical Physics and Biomedical Engineering, June 7-12, 2015, Toronto, Canada, vol. 51, D. A. Jaffray, Ed. Cham: Springer International Publishing, 2015, pp. 1146-1149.

[33] A. M. Gal, A. D. Chan, and D. C. Hay, "Investigating the seated double poilng 
cycle: identifying baseline measures for the preparation phase," in ISBS-Conference Proceedings Archive, 2016, vol. 1.

[34] T. Stöggl and H.-C. Holmberg, "Force interaction and 3D pole movement in double poling: Force interaction and 3D in double poling," Scand. J. Med. Sci. Sports, vol. 21, no. 6, pp. e393-e404, Dec. 2011.

[35] Chris Kirtley, Clinical gait analysis theory and practice. Edinburgh: Churchill Livingstone, 2006.

[36] C. H. Yeow, P. V. S. Lee, and J. C. H. Goh, "Sagittal knee joint kinematics and energetics in response to different landing heights and techniques," The Knee, vol. 17, no. 2, pp. 127-131, Mar. 2010.

[37] N. P. Linthorne, "Analysis of standing vertical jumps using a force platform," Am. J. Phys., vol. 69, no. 11, pp. 1198-1204, Nov. 2001.

[38] M. S. Ferrara and R. W. Davis, "Injuries to elite wheelchair athletes," Int. Med. Soc. Paraplegia, vol. 28, pp. 335-341, 1990.

[39] Wikipedia, "Sledge Hockey," 07-Sep-2016. [Online]. Available: https://en.wikipedia.org/wiki/Sledge_hockey.

[40] Hockey Canada, "2015-2016 National sledge team," National sledge team. [Online]. Available: http://www.hockeycanada.ca/en-ca/TeamCanada/Men/Sledge.aspx.

[41] Walter R. Frontera, Stanley A. Herring, Timonthy P. Young, Lyle J. Micheli, and Julie K. Silver, Clinical sports medicine: medical management and rehabilitation. Philadelphia, PA.: Saunders, 2007.

[42] H.-C. Holmberg, S. Lindinger, T. Stoggl, and E. Muller, "Biomechanical analysis of double poling in elite cross-country skiers:," Med. Sci. Sports Exerc., vol. 37, no. 5, pp. 807-818, May 2005.

[43] K. Lomond, "A kinematic analysis of sledge hockey propulsion," Undergraduate, Acadia University, 2003.

[44] M. Bernardi, T. Janssen, L. Bortolan, B. Pellegrini, G. Fischer, and F. Schena, "Kinematics of cross-country sit skiing during a Paralympic race," J. Electromyogr. Kinesiol., vol. 23, no. 1, pp. 94-101, Feb. 2013.

[45] T. Stöggl, E. Müller, M. Ainegren, and H.-C. Holmberg, "General strength and kinetics: fundamental to sprinting faster in cross country skiing?: Strength and kinetics in cross country skiing," Scand. J. Med. Sci. Sports, vol. 21, no. 6, pp. 791803, Dec. 2011.

[46] A. Mital, A. Kilbom, and S. Kumar, Ergonomics Guidelines and Problem Solving, 1st ed. Oxford, UK: Elsevier Science Ltd, 2000.

[47] G. Y. Millet, M. D. Hoffman, R. B. Candau, and P. S. Clifford, "Poling forces during roller skiing: effects of technique and speed:," Med. Sci. Sports Exerc., vol. 30, no. 11, pp. 1645-1653, Nov. 1998.

[48] T. L. StöGgl and E. MüLler, "Kinematic Determinants and Physiological Response of Cross-Country Skiing at Maximal Speed:," Med. Sci. Sports Exerc., vol. 41, no. 7, pp. 1476-1487, Jul. 2009.

[49] J. Nilsson, P. Tveit, and O. Eikrehagen, "Effects of speed on temporal patterns in freestyle cross-country skiing," Unpubl. Draft Manuscr., 2002.

[50] J. Nilsson, V. Jakobsen, P. Tveit, and O. Eikrehagen, "Skiing: Pole length and ground reaction forces during maximal double poling in skiing," Sports Biomech., 
vol. 2, no. 2, pp. 227-236, Jul. 2003.

[51] S. J. Lindinger, T. StöGgl, E. MüLler, and H.-C. Holmberg, "Control of speed during the double Poling technique performed by elite cross-country skiers:," Med. Sci. Sports Exerc., vol. 41, no. 1, pp. 210-220, Jan. 2009.

[52] J. Mikkola, M. S. Laaksonen, H.-C. Holmberg, A. Nummela, and V. Linnamo, "Changes in performance and poling kinetics during cross-country sprint skiing competition using the double-poling technique," Sports Biomech., vol. 12, no. 4, pp. 355-364, Nov. 2013.

[53] M. S. Ferrara, W. E. Buckley, D. G. Messner, and J. Benedict, "The injury experience and training history of the competitive skier with a disability," Am. J. Sports Med., vol. 20, no. 1, pp. 55-60, 1992.

[54] S. J. Lindinger and H.-C. Holmberg, "How do elite cross-country skiers adapt to different double poling frequencies at low to high speeds?," Eur. J. Appl. Physiol., vol. 111, no. 6, pp. 1103-1119, Jun. 2011.

[55] M. Häyrinen et al., "Match analysis of elite ice sledge hockey in Paralympics 2010," J. Quant. Anal. Sports, vol. 7, no. 3, Jan. 2011.

[56] M. Kudláček, I. Dalbesio, Z. Janečka, Y. Vanlandewijck, and E. Beckman, "The analysis of individual skills of ice sledge hockey players at the Torino 2006 Paralympic tournament," Eur. J. Adapt. Phys. Act., vol. 2, no. 2, 2009.

[57] E. Beckman, M. Kudláček, Y. Vanlandewijck, and others, "Development of a skills observation protocol for sledge ice hockey-pilot study," Acta Univ. Palacki. Olomuc. Gymnica, vol. 37, pp. 45-50, 2007.

[58] B. Molik et al., "Game performance in ice sledge hockey: An exploratory examination into type of disability and anthropometric parameters," Clin. J. Sport Med., p. 1, Jan. 2012.

[59] Ø. Sandbakk, M. Spencer, G. Ettema, S. B. Sandbakk, K. Skovereng, and B. Welde, "The physiology and biomechanics of upper-body repeated sprints in ice sledge hockey," Int. J. Sports Physiol. Perform., vol. 9, no. 1, pp. 77-84, Jan. 2014.

[60] Ø. Sandbakk, M. Hansen, G. Ettema, and B. Rønnestad, "The effects of heavy upper-body strength training on ice sledge hockey sprint abilities in world class players," Hum. Mov. Sci., vol. 38, pp. 251-261, Dec. 2014.

[61] K. Skovereng, G. Ettema, B. Welde, and Ø. Sandbakk, "On the relationship between upper-body strength, power, and sprint performance in ice sledge hockey:," $J$. Strength Cond. Res., vol. 27, no. 12, pp. 3461-3466, Dec. 2013.

[62] C. Worden-Rogers, "Effect of sledge hockey configuration on sledge hockey performance," M.Sc., The University of Western Ontario, Waterloo, ON, 2012.

[63] K. Wynnyk and N. Spencer-Cavaliere, "Children's social relationships and motivation in sledge hockey," Adapt. Phys. Act. Q., vol. 30, no. 4, pp. 299-316, Oct. 2013.

[64] K. E. Wynnyk, "Exploring social relationships and children with disabilities' motivation to participate in sledge hockey," M.A., University of Alberta, 2013.

[65] J. Nilsson, F. Tinmark, K. Halvorsen, and A. Arndt, "Kinematic, kinetic and electromyographic adaptation to speed and resistance in double poling cross country skiing," Eur. J. Appl. Physiol., vol. 113, no. 6, pp. 1385-1394, Jun. 2013.

[66] Dictionary.com, "biomechanics," Dictionary.com Unabridged. Source location: Random House, Inc, http://www.dictionary.com/browse/biomechanics. 
[67] G. Robertson, G. Caldwell, J. Hamill, G. Kamen, and S. Whittlesey, Research methods in biomechanics, 1st ed. Champaign, IL: Human Kinetics, 2004.

[68] G. Wu et al., "ISB recommendation on definitions of joint coordinate systems of various joints for the reporting of human joint motion-Part II: shoulder, elbow, wrist and hand," J. Biomech., vol. 38, no. 5, pp. 981-992, May 2005.

[69] J. Richards, Biomechanics in clinic and research. Edinburgh: Churchill Livingstone, 2008.

[70] D. L. Benoit, D. K. Ramsey, M. Lamontagne, L. Xu, P. Wretenberg, and P. Renström, "Effect of skin movement artifact on knee kinematics during gait and cutting motions measured in vivo," Gait Posture, vol. 24, no. 2, pp. 152-164, Oct. 2006.

[71] A. Barré, B. M. Jolles, N. Theumann, and K. Aminian, "Soft tissue artifact distribution on lower limbs during treadmill gait: Influence of skin markers' location on cluster design," J. Biomech., vol. 48, no. 10, pp. 1965-1971, Jul. 2015.

[72] P. Kasyan, "Kinetics 2009 lecture 2 with torque," 2009.

[73] Slashme, Human anatomy planes.jpg. 2014.

[74] T. Ogele, Body movements I.jpg. 2013.

[75] M. Ruiz Villarreal, Human arm bones diagram.svg. 2007.

[76] T. McBride and S. Kalogrianitis, "Dislocations of the shoulder joint," Trauma, vol. 14, no. 1, pp. 47-56, Jan. 2012.

[77] W. T. Simonet and R. H. Cofield, "Prognosis in anterior shoulder dislocation," Am. J. Sports Med., vol. 12, no. 1, pp. 19-24, 1984.

[78] J. W. Ufberg, G. M. Vilke, T. C. Chan, and R. A. Harrigan, “Anterior shoulder dislocations: Beyond traction-countertraction,” J. Emerg. Med., vol. 27, no. 3, pp. 301-306, Oct. 2004.

[79] I. R. Murray, I. Ahmed, N. J. White, and C. M. Robinson, "Traumatic anterior shoulder instability in the athlete: Anterior shoulder instability in the athlete," Scand. J. Med. Sci. Sports, vol. 23, no. 4, pp. 387-405, Aug. 2013.

[80] P. Randelli and E. Taverna, "Primary anterior shoulder dislocation in young athletes: fix them!," Knee Surg. Sports Traumatol. Arthrosc., vol. 17, no. 12, pp. 1404-1405, Dec. 2009.

[81] A. Rumian, D. Coffey, S. Fogerty, and R. Hackney, "Acute first-time shoulder dislocation," Orthop. Trauma, vol. 25, no. 5, pp. 363-368, 2011.

[82] M. S. Ferrara and C. L. Peterson, "Injuries to athletes with disabilities," Sports Med., vol. 30, no. 2, pp. 137-143, 2000.

[83] A. R. Davy and S. J. Drew, "Management of shoulder dislocation: Are we doing enough to reduce the risk of recurrence?," Injury, vol. 33, no. 9, pp. 775-779, 2002.

[84] S. R. Dubowsky, J. Rasmussen, S. A. Sisto, and N. A. Langrana, "Validation of a musculoskeletal model of wheelchair propulsion and its application to minimizing shoulder joint forces," J. Biomech., vol. 41, no. 14, pp. 2981-2988, Oct. 2008.

[85] K. Kulig et al., "The effect of level of spinal cord injury on shoulder joint kinetics during manual wheelchair propulsion," Clin. Biomech., vol. 16, no. 9, pp. 744-751, 2001.

[86] J. L. Mercer, M. Boninger, A. Koontz, D. Ren, T. Dyson-Hudson, and R. Cooper, "Shoulder joint kinetics and pathology in manual wheelchair users," Clin. Biomech., vol. 21, no. 8, pp. 781-789, Oct. 2006. 
[87] J. L. Collinger et al., "Shoulder biomechanics during the push phase of wheelchair propulsion: A multisite study of persons with paraplegia," Arch. Phys. Med. Rehabil., vol. 89, no. 4, pp. 667-676, Apr. 2008.

[88] M. M. B. Morrow, W. J. Hurd, K. R. Kaufman, and K.-N. An, "Shoulder demands in manual wheelchair users across a spectrum of activities," J. Electromyogr. Kinesiol., vol. 20, no. 1, pp. 61-67, Feb. 2010.

[89] R. Chowdhury, M. Reaz, M. Ali, A. Bakar, K. Chellappan, and T. Chang, "Surface electromyography signal processing and classification techniques," Sensors, vol. 13, no. 9, pp. 12431-12466, Sep. 2013.

[90] C. J. De Luca, “The use of surface electromyography in biomechanics," J. Aplpied Biomech., vol. 13, pp. 135-163, 1997.

[91] C. J. De Luca, "Decomposition of surface EMG signals," J. Neurophysiol., vol. 96, no. 3, pp. 1646-1657, Jan. 2006.

[92] C. J. De Luca, "Surface electromyography: Detection and recording." DelSys Inc., 2002.

[93] M. B. I. Reaz, M. S. Hussain, and F. Mohd-Yasin, "Techniques of EMG signal analysis: detection, processing, classification and applications," Biol. Proced. Online, vol. 8, no. 1, pp. 11-35, Dec. 2006.

[94] M. C. Garcia and T. M. M. Vieira, "Surface electromyography: Why, when and how to use it," Rev. Andal. Med. Deporte, no. 1, pp. 17-28, 2011.

[95] Andreea Macoveiciuc, "Fitness basic: Beginner upper body exercises," Rivertea, 30-Jan-2014. [Online]. Available: http://www.rivertea.com/blog/fitness-basicsbeginner-upper-body-exercises/. [Accessed: 11-Nov-2016].

[96] D. A. Winter, Biomechanics and motor control of human movement, 4 th ed. New Jersey: John Wiley \& Sons, Inc., 2005.

[97] S. L. Delp et al., "OpenSim: Open-Source Software to Create and Analyze Dynamic Simulations of Movement," IEEE Trans. Biomed. Eng., vol. 54, no. 11, pp. 19401950, Nov. 2007.

[98] C. L. Vaughan, "Computer Simulation of Human Motion in Sports Biomechanics," Exerc. Sports Sci. Rev., vol. 12, no. 1, pp. 373-398, Jan. 1984.

[99] M. Panjabi, "Validation of mathematical models," J. Biomech., vol. 12, no. 3, p. 238, 1979.

[100] W. L. Bauer, "A manually operated biomechanical model of an internally energized self-oscillatory system," in Ninth International Congress of

Biomechanics, Waterloo, ON, 1985, pp. 383-388.

[101] Maplesoft, Maple. Japan: Cybernet System Co. Ltd.

[102] National Centre for Stimulation in Rehabilitation Research, OpenSim. 2010.

[103] Vicon Motion Systems Ltd., Nexus. U.K.: Vicon Motion Systems Ltd.

[104] A. M. Gal, A. D. C. Chan, and D. C. Hay, "Propulsion in sledge hockey: A biomechanical analysis to define gait," presented at the Own the Podium Sports Innovation Summit, Montreal, Canada, 2014.

[105] A. M. Gal, A. D. C. Chan, and D. C. Hay, "Shoulder joint produced weightbearing mobility: Defining gait," presented at the Women in Science and Engineering National Conference, Toronto, Candaa, 2015.

[106] A. M. Gal, A. D. C. Chan, and D. C. Hay, "Baseline impact reaction forces in a seated downward short pole free-drop: A biomechanical investigation of elbow 
angle upon impact in the sport of sledge hockey," presented at the Own the Podium Sports Innovation Summit, Toronto, Canada, 2015.

[107] Bertec Corp., "Bertec Force Plate," Bertec, 2016. [Online]. Available: http://bertec.com/products/force-plates/.

[108] MathWorks, MATLAB. The Mathworks Inc., 1994.

[109] Wikipedia, "Applied mathematics," Wikipedia the free encyclopedia, 03-Mar2017. [Online]. Available: https://en.wikipedia.org/wiki/Applied_mathematics. [Accessed: 20-Apr-2017].

[110] F. M. Impellizzeri, "Correlations between physiological variables and performance in high level cross country off road cyclists," Br. J. Sports Med., vol. 39, no. 10, pp. 747-751, Oct. 2005.

[111] Canadian Society for Exercise Physiology, "Physical activity readiness questionnaire." 2016.

[112] Delsys, "Delsys wearable sensors for movement science," 2016. [Online]. Available: http://www.delsys.com/educational-resources/knowledgecenter/publications/. [Accessed: 13-Sep-2015].

[113] Seniam, "Seniam." [Online]. Available: http://www.seniam.org/.

[114] K. Saitou, T. Masuda, D. Michikami, R. Kojima, and M. Okada, "Innervation zones of the upper and lower limb muscles estimated by using multchannel surface EMG," J. Hum. Ergol. (Tokyo), vol. 29, pp. 35-52, 2000.

[115] H. J. Hermens, B. Freriks, C. Disselhorst-Klug, and G. Rau, "Development of recommendations for SEMG sensors and sensor placement procedures," $J$. Electromyogr. Kinesiol., vol. 10, no. 5, pp. 361-374, Oct. 2000.

[116] K. Häkkinen et al., "Changes in agonist-antagonist EMG, muscle CSA and force during strength training in middle-aged and older people," J Appl Physiol, vol. 84, pp. 1341-1349, 1998.

[117] Comfy Splints, “Orthosis locking elbow brace," Comfy Splints, 2009. [Online]. Available: http://www.comfysplints.com/product_info.php?cPath=44\&products_id=80.

[118] Kistler Instrument Corp., "Kistler force plate," Kistler: measure. analyze. innovate., 2016. [Online]. Available: https://www.kistler.com/ca/en/.

[119] BTS Bioengineering, "BTS Wireless sEMG," BTS Bioengineering, 2016. [Online]. Available: http://www.btsbioengineering.com/.

[120] A. M. Gal, A. D. C. Chan, and D. C. Hay, "Differences between sagittal plane static start and mid-cycle stroke biomechanics during skating for the sport of sledge hockey: Task naïve population," presented at the Own the Podium Sports Innovation Summit, Calgary, Canada, 2016.

[121] A. M. Gal, A. D. C. Chan, and D. C. Hay, "Investigation of the relationship between peak impact and push-off reaction forces, and potential upper limb overuse/overloading injuries introduced from skating in the sport of sledge hockey," presented at the Own the Podium Sports Innovatin Summit, Calgary, Canada, 2016.

[122] M. S. Ferrara and R. W. Davis, "Injuries to elite wheelchair athletes," Int. Med. Soc. Paraplegia, vol. 28, pp. 335-341, 1990.

[123] V. Sholukha, S. Van Sint Jan, O. Snoeck, P. Salvia, F. Moiseev, and M. Rooze, "Prediction of joint center location by customizable multiple regressions: Application to clavicle, scapula and humerus," J. Biomech., vol. 42, no. 3, pp. 319- 
324, Feb. 2009.

[124] J. G. Webster, "Basic concepts of medical instrumentation," Med. Instrum. Appl. Des., pp. 10-11.

[125] A. Murphy and H. Knipe, "Acromiohumeral interval," Radiopaedia.org, 01-Dec2016. [Online]. Available: https://radiopaedia.org/articles/acromiohumeral-interval. [Accessed: 01-Dec-2016].

[126] G. R. Milner and J. L. Boldsen, "Humeral and Femoral Head Diameters in Recent White American Skeletons: Humeral and femoral head diameters," J. Forensic Sci., vol. 57, no. 1, pp. 35-40, Jan. 2012.

[127] G. . Nicholson, D. A. Goodman, E. L. Flatow, and L. U. Bigliani, "The acromion: Morphologic condtion and age-related changes. A study of 420 scapulas," $J$ Shoulder Elb. Surg, vol. 5, no. 1, pp. 1-11, Feb. 1996.

[128] S. Plagenhoef, F. G. Gaynor Evans, and T. Abdelnour, "Anatomical data for analyzing human motion,” Res Q Exerc Sport, vol. 54, pp. 169-178, 1983. 